\title{
DETECTING SOIL EROSION IN SEMI-ARID MEDITERRANEAN ENVIRONMENTS USING SIMULATED ENMAP DATA
}

\author{
Ashley H. Bracken \\ B.Sc. University of Lethbridge, 2008
}

\author{
A Thesis \\ Submitted to the School of Graduate Studies \\ of the University of Lethbridge \\ in Partial Fulfillment of the \\ Requirements of the Degree

\section{MASTER OF SCIENCE} \\ Department of Geography \\ University of Lethbridge \\ LETHBRIDGE, ALBERTA CANADA
}

(C)Ashley H. Bracken, 2014 


\section{DETECTING SOIL EROSION IN SEMI-ARID MEDITERRANEAN ENVIRONMENTS USING SIULATED ENMAP DATA}

\section{ASHLEY H. BRACKEN}

Date of Defense: August 26, 2014

Dr. Karl Staenz

Co-Supervisor

Dr. Craig Coburn

Co-Supervisor

Dr. Nadia Rochdi

Thesis Examination

Committee Member

Dr. Rene Barendregt

Thesis Examination

Committee Member

Dr. Kevin McGeough

Chair, Thesis

Examination

Committee
Professor

$\mathrm{PhD}$

Associate Professor

$\mathrm{PhD}$

Adjunct Assistant Professor

PhD

Professor

$\mathrm{PhD}$

Associate Professor

$\mathrm{PhD}$ 


\begin{abstract}
Soil is an essential nature resource. Management of this resource is vital for sustainability and the continued functioning of earths atmospheric, hydrospheric and lithospheric functioning. The assessment and continued monitoring of surface soil state provides the information required to effectively manage this resource. This research used a simulated Environmental Mapping and Analysis Program (EnMAP) hyperspectral image cube of an agricultural region in semi- arid Mediterranean Spain to classify soil erosion states. Multiple Endmember Spectral Mixture Analysis (MESMA) was used to derive within pixel fractions of eroded and accumulated soils. A Classification of the soil erosion states using the scene fraction outputs and digital terrain information. The information products generated in this research provided an optimistic outlook for the applicability of the future EnMAP sensor for soil erosion investigations in semi-arid Mediterranean environments. Additionally, this research verifies that the launch of the EnMAP satellite sensor in 2018 will provide the opportunity to further improve the monitoring of earth finite soil resources.
\end{abstract}




\section{ACKNOWLEDGEMENTS}

I would like to thank my supervisors, Dr. Karl Staenz and Dr. Craig Coburn, and my committee members, Dr. Nadia Rochdi and Dr. Rene Barendregt. I would also like to thank Dr. Sabine Chabrillat, Dr. Karl Segl, Dr. Hermann Kaufmann and the entire working group in section 1.4 at the GFZ, Potsdam, Germany. Thanks also go to Dr. Thomas Schmid and the working group at CIEMAT, Madrid, Spain. Thanks to the University of Lethbridge and NSERC create AMETHYST program for funding. I would also like to thank the Alberta Terrestrial Imaging Centre. Lastly, to my friends and my mum, thank you. 


\section{TABLE OF CONTENTS}

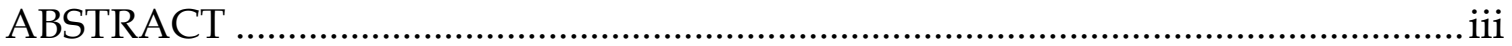

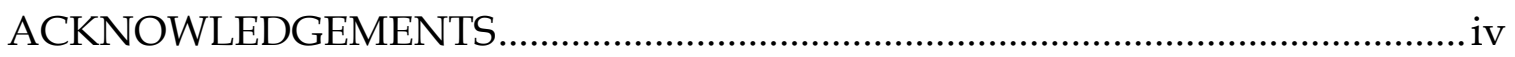

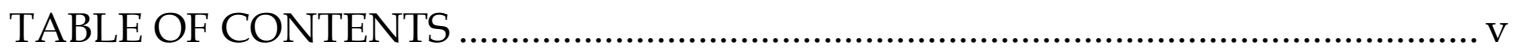

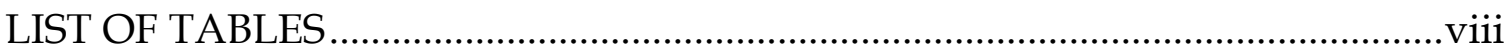

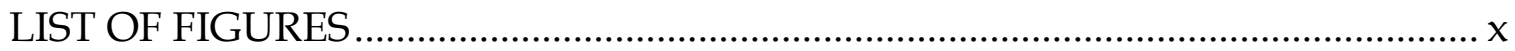

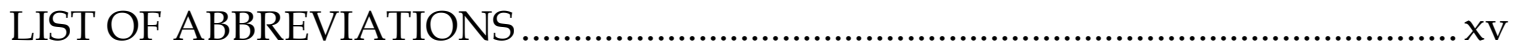

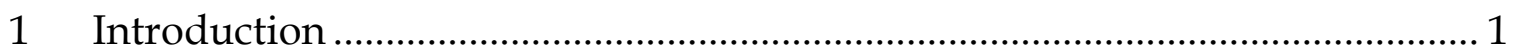

1.1 Remote Sensing of Soil Erosion ................................................................. 2

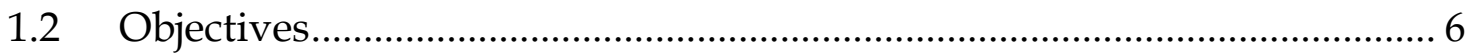

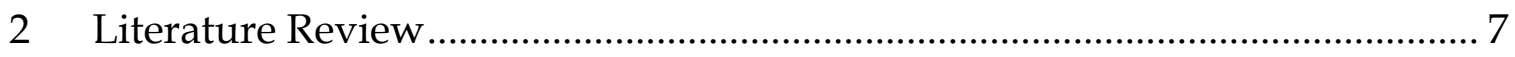

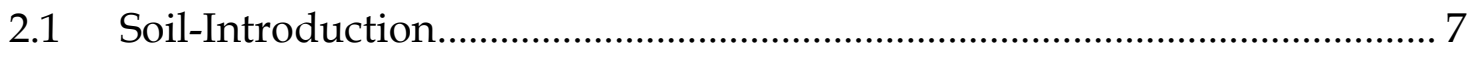

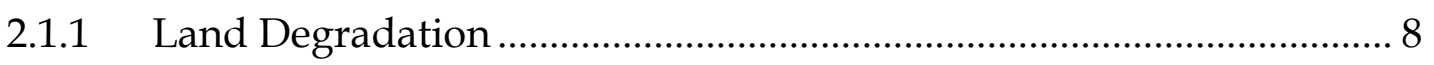

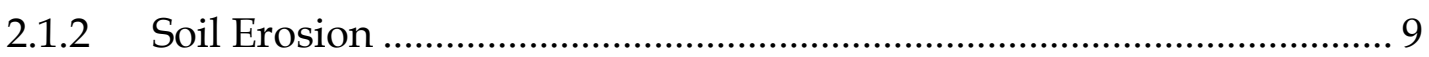

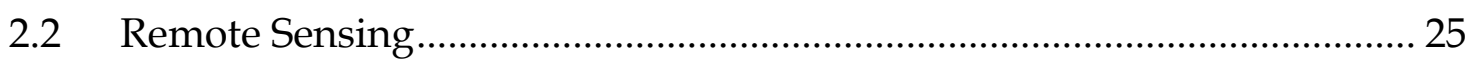

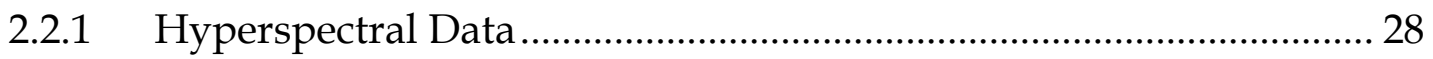

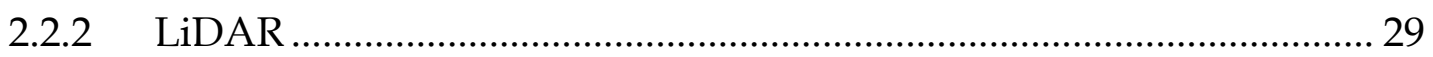

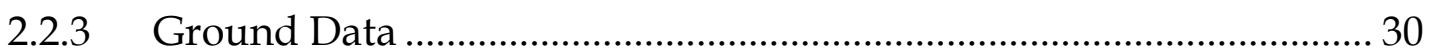

2.3 Hyperspectral Data Pre-Processing ........................................................... 32

2.3.1 Sensor Radiometric Calibration ....................................................... 32

2.3.2 Atmospheric correction ................................................................... 34

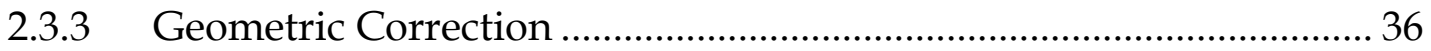

2.3.4 Bidirectional Reflectance Distribution Function (BRDF) .................. 42

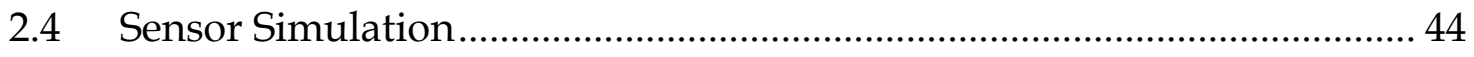


2.4.1 Sensor Simulations - Future Sensors ………………………………..... 45

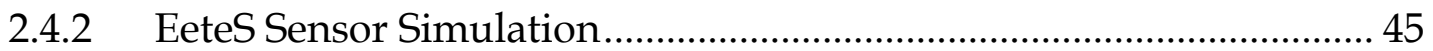

2.5 Hyperspectral Image Processing ................................................................ 46

2.5.1 Linear Spectral Unmixing ................................................................... 47

2.6 Remote Sensing of Soils ............................................................................ 53

2.6.1 Soil Imaging Spectroscopy ……………………................................... 54

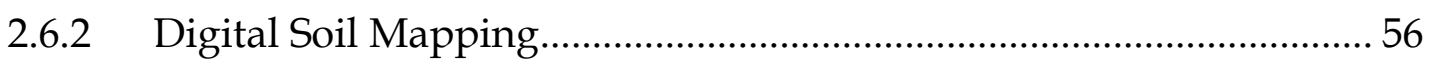

2.7 Terrain Analysis for Soil Applications ........................................................ 62

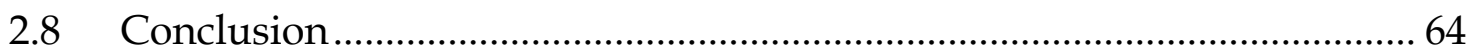

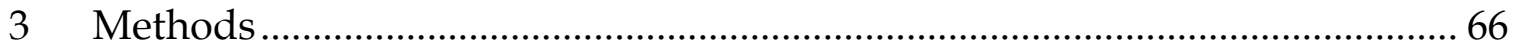

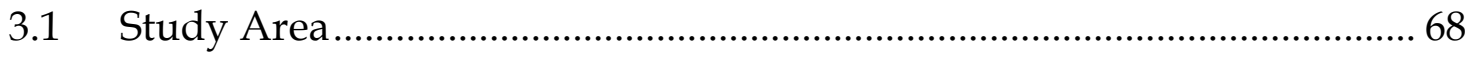

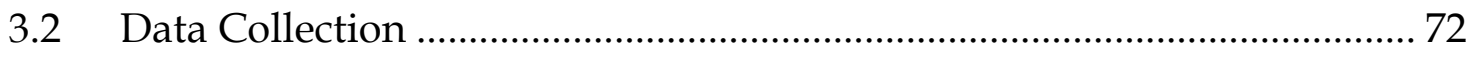

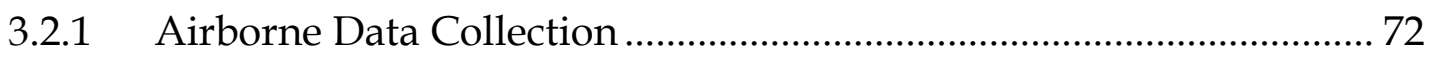

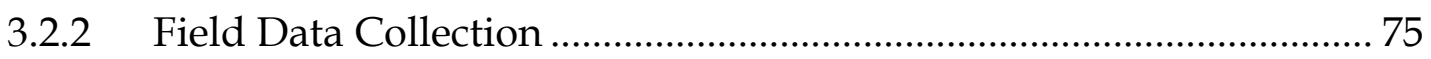

3.3 Hyperspectral Image Pre-processing ……………………………............ 80

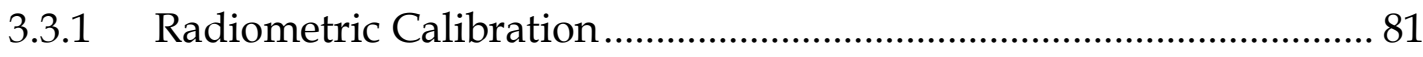

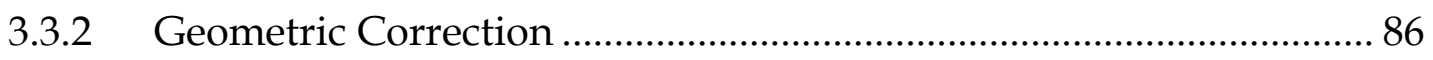

3.3.3 Atmospheric Correction....................................................................... 91

3.3.4 Cross-Track Illumination Correction .................................................... 93

3.3.5 Mosaicking ....................................................................................... 96

3.3.6 Minimum Noise Fraction ................................................................... 99

3.3.7 Empirical Line Correction.................................................................... 100

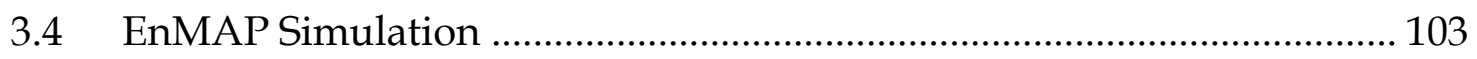

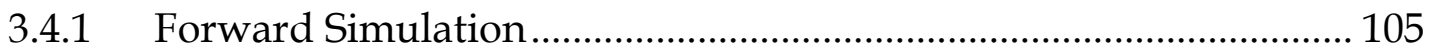

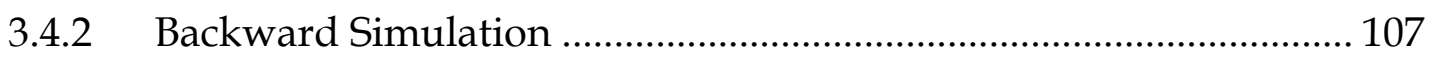

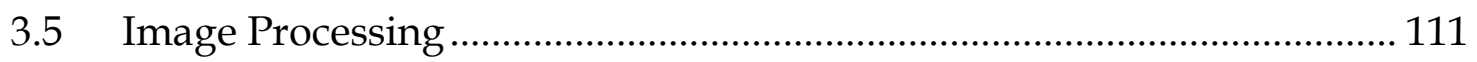




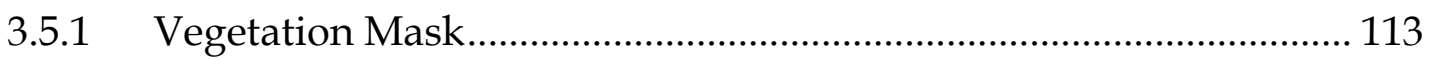

3.5.2 Soil Unmixing .............................................................................. 120

3.5.3 Terrain Analysis ................................................................................... 124

3.5.4 Spectral Soil Erosion Class Generation ............................................... 127

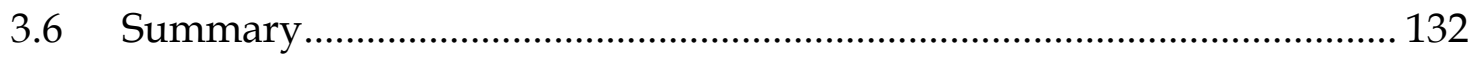

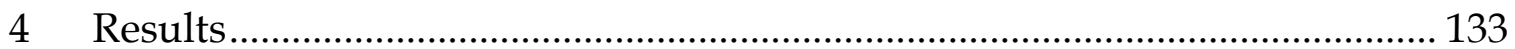

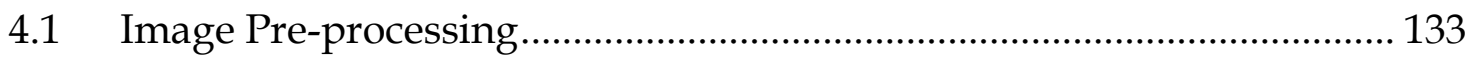

4.1.1 Radiometric Correction - Destriping ………………....................... 133

4.1.2 Geometric Correction ...................................................................... 137

4.1.3 Atmospheric Correction.................................................................. 141

4.1.4 Cross-Track Illumination Correction ................................................. 149

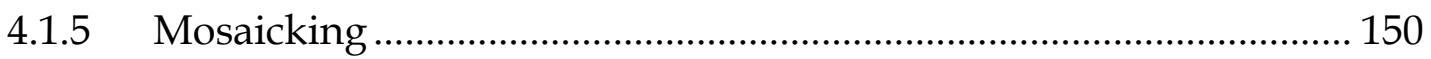

4.1.6 Minimum Noise Fraction ................................................................. 150

4.1.7 Empirical Line Correction................................................................... 151

4.2 EnMAP Simulation .............................................................................. 153

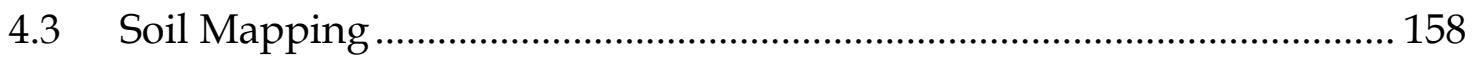

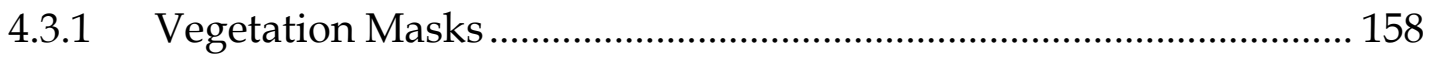

4.3.2 Soil Erosion Status Classification......................................................... 165

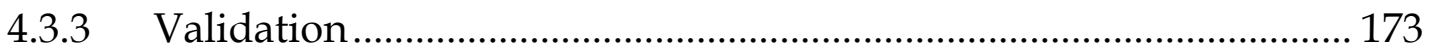

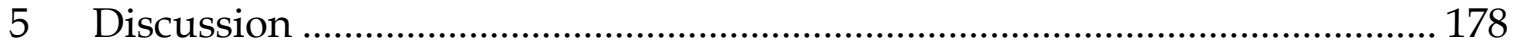

$5.1 \quad$ Hyperspectral Image Pre-processing ……................................................ 178

5.2 Image Processing ................................................................................ 184

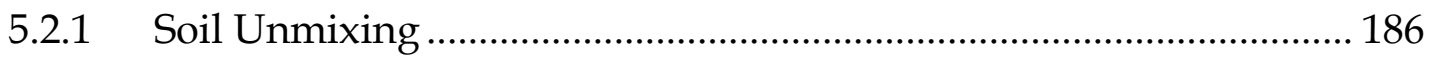

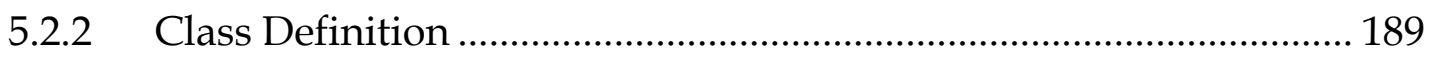

5.2.3 Accuracy Assessment ....................................................................... 190

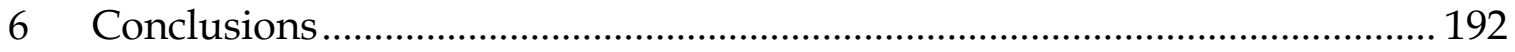

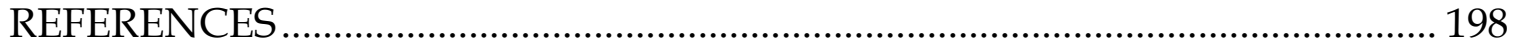




\section{LIST OF TABLES}

Table 2.1: Description of topographic variables...............................63

Table 3.1: Geographic coordinates (Zone 30N, ERTS 89 datum) for the Camarena study site...............................................68

Table 3.2: $\quad$ Characteristics of the AISA Eagle and AISA Hawk data. SSI = Spectral Sampling Interval, FOV = Field Of View...................74

Table 3.3: Characteristics of the Leica ALS50 (II) LiDAR data....................74

Table 3.4: Mineralogy, texture and Munsell soil colour (dry) characteristics of six sampling sites located in the SU field site. The field sites are titled based on their field site location (SU) and the order they were collected (1-6). The order in which they appear in the Table is based on their soil erosion stage ..........................................

Table 3.5: Details of the flight and solar geometry parameters for the image acquisition. Flight lines 22001 and 22008 are the across-track flight lines. This can be seen by their heading directions in comparisons to the other flight lines............................................99

Table 3.6: Performance characteristics of the future EnMAP sensor. Information was gathered from Kaufmann et al. (2012) .............110

Table 3.7: The elevation, curvature, upslope contributing area and slope values collected for each of the SU field sites. The definition of curvature is taken from Wilson \& Gallant (2000)...................126

Table 3.8: Maximum and minimum fraction values, means and Standard Deviation (Stdev) values collected for each erosion state from each fraction image..................................................130

Table 3.9: Maximum and minimum fraction values used to define the erosion classes................................................................131

Table 4.1: $\quad$ Percent of the total AISA scene that has fraction values below zero and above one for each endmember. 160 
Table 4.2: $\quad$ Percent of the RMS error image that falls in to each class. The class values are selected based on Haboudane et al. (2002)...............161

Table 4.3: Percent of the total EnMAP scene that has fraction values below zero and above one for each endmember.........................163

Table 4.4: $\quad$ Percent of the RMS error image that falls in to each class. The class values are selected based on Haboudane et al. (2002)...............164

Table 4.5: Percent of the total AISA scene that has fraction values below zero and above 1 for each endmember in the soils unmixing............166

Table 4.6: Percent of the RMS error image that falls in to each class for the AISA soils unmixing. The class values are selected based on Haboudane et al. (2002)..........................................166

Table 4.7: Eroded and accumulated fraction values from each field location within the AISA image.................................................167

Table 4.8: Percent of the total scene that has fraction values below zero and above 1 for each endmember in the EnMAP soils unmixing.......171

Table 4.9: $\quad$ Percent of the RMS error image that falls in to each RMSE class for the EnMAP soils unmixing. The class values are selected based on Haboudane et al. (2002)..........................................171

Table 4.10: Confusion matrix generated for the EnMAP final soil erosion state map..........................................................

Table 5.1: $\quad$ The eroded fraction values from each field location and the calcite content measured from soils samples collected from the field sites 188 


\section{LIST OF FIGURES}

Figure 2.1 Comparison of green vegetation spectra, dry vegetation spectra and

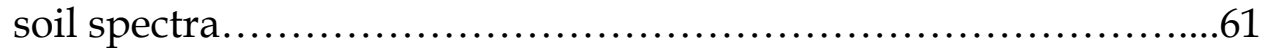

Figure 3.1 The Camarena study site in relation to Madrid, Spain. The Camarena study site is outlined in red. The dots within the Camarena study site highlight the field sampling sites collected during the August 2011 field campaign.............................67

Figure 3.2: A typical soil profile in the study with associated soil characteristics. The A hoizon is characterized by moderate organic material content and coarse texture. The $\mathrm{B}$ horizon is fine textured and is rich in $\mathrm{Fe}$ oxides. The $\mathrm{Ck}$ horizon is rich in carbonates and coarse textured. The $\mathrm{C}$ horizon is composed of arkosic rock. .71

Figure 3.3: A preliminary mosaic of the eight flight lines acquired on August 8th, 2011. A = Main flight lines; $\mathrm{B}=$ Perpendicular flight lines

Figure 3.4: Images of the 8 surface covers used for field data collection. a) Fallow land. b) Rain-fed cereal cultivation. c) Vineyard. d) Tillage. e) Organic matter on surface. .78

Figure 3.5: Sampling schematic used in the Camarena field site. .79

Figure 3.6: Flow chart of the preprocessing steps performed on the AISA Eagle and AISA Hawk data

Figure 3.7: An example of the vertical striping found in the AISA Hawk data. The striping shown is inconsistent in magnitude along the column. This is representative of all of the striping, excluding the dropped columns, in the AISA Hawk image. ...83

Figure 3.8: Shows the four types of striping features identified in the destriping process. From left to right: local maxima (top and bottom), local minima, left linear increase, right linear increase...................................................... 84

Figure 3.9: Two field location spectra converted to radiance and the corresponding image radiance spectra used to define the jump characteristics. The jump is located between $978 \mathrm{~nm}$ and $1096 \mathrm{~nm}$. This is where the image and field radiance spectra differ greatly. 
This is the wavelength range where the image spectra were corrected.

Figure 3.10: Change in sun position in relation to Camarena, Toledo, Spain over the duration of the flight campaign.

Figure 3.11: Flight line subsets used for the mosaicking of the Camarena study site.

Figure 3.12: A horizontal subset of the mosaicked flight lines (top) and a close up on a seam line (bottom) in the image subset shown on the top. The close up highlights the brightness variation between the two flight lines. .98

Figure 3.13: Linear regression used to derive the gain and offset coefficients for the ELC in one band. In this image, the points in the plot represent the spectra being used for the ELC. The point corresponding to $X_{1}$, $Y_{1}$ is a bright target and the one corresponding to $X_{2}, Y_{2}$ is a dark target. The positions of the points in the plot are determined by the value of the field spectra and the value of the corresponding image spectra in that particular band. The gain is calculated using $\frac{\mathrm{Y}_{1}-\mathrm{Y}_{2}}{\mathrm{X}_{1}-\mathrm{X}_{2}}$ 101

Figure 3.14: Field spectra selected for the ELC of the image data. 103

Figure 3.15: EeteS simulation process from input reflectance data to output EnMAP reflectance data. 104

Figure 3.16: AISA SU subset (top) and EnMAP SU subset (bottom). Both images have the SU-field sampling sites highlighted with labeled red dots.

Figure 3.17: Endmember spectra used for unmixing the AISA data with the green vegetation endmember (top), soil endmembers (bottom), and dry vegetation endmembers (middle). 116

Figure 3.18: Endmember spectra used for unmixing of the EnMAP data with the green vegetation endmembers (top), soil endmembers (bottom), and dry vegetation endmembers (middle). .117

Figure 3.19: RGB composite of the endmember fractions for the AISA (top) and EnMAP (bottom). $R=$ dry vegetation, $G=$ green vegetation and $B=$ soil. 
Figure 3.20: AISA (top) and EnMAP (bottom) images with the vegetation mask overlaid. Only visible in these images are the soil dominated pixels.

Figure 3.21: Spectra collected for both the eroded (blue) and accumulated (red) endmembers used for unmixing the AISA image. 122

Figure 3.22: Spectra collected for both the eroded (blue) and accumulated (red) endmembers used for unmixing the EnMAP image.................123

Figure 3.23: AISA accumulated fraction map (top left), AISA eroded fraction map (top right), EnMAP accumulated fraction map (bottom left) and EnMAP eroded fraction map (bottom right). In all images, brighter areas represent higher fraction values

Figure 3.24: SU site DEM (top) and a 3D view of the SU site DEM (bottom). The SU field sites are highlighted with red dots.... 126

Figure 3.25: Images of curvature (top), upslope contributing area (middle) and slope (bottom) 127

Figure 3.26: Contour map of the SU site(top) with the SU field locations indicated with black crosses and the transect line shown in red with elevation profile of the transect (bottom). .129

Figure 4.1: Example from before (left) and after (right) the destriping procedure. .134

Figure 4.2: Comparison of a horizontal profile from flight line 22005 at 1443.8 $\mathrm{nm}$ before and after destriping. The horizontal profile extends the width of the flight line. The red spikes are the result of striping. Their locations show the columns where striping occurs. 135

Figure 4.3: The artifact in flight line 22009, column 35 at $2232.4 \mathrm{~nm}$. The artifact is circled in red.....................................................

Figure 4.4: Individual GCP error increased towards the outer edges of the flight lines. In this example, GCP 3 will have a substantially larger

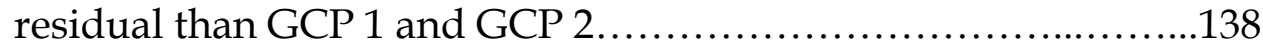

Figure 4.5: A comparison of the pre-and post- jump corrected spectra for the SU2 field location. 140

Figure 4.6: A histogram of the illumination change in the scene over the duration of the flight campaign. Negative values along the $\mathrm{X}$-axis 
represent increases in illumination and positive values along the $\mathrm{X}$ axis represent decreases in illumination...........................143

Figure 4.7: Comparison between the estimated ATCOR spectra and the field spectra for the SU1 through SU6 field sites..........................145

Figure 4.8: Comparison of the field and AISA spectra from location SU2. The vertical line located at $2349 \mathrm{~nm}$ indicates the calcite feature present in the SU2 field spectra. The AISA spectra at this location contains a substaintial amount of noise and presents no clear calcite absorption feature.............................................146

Figure 4.9: A comparison of the $\mathrm{CO}_{2}$ absorption features between the AISA data and the simulated MODTRAN spectrum. The AISA spectrum was collected from a road surface. The vertical lines in red and blue identify the center wavelength position of the MODTRAN and AISA $\mathrm{CO}_{2}$ absorption features, respectively.....................148

Figure 4.10: The x-profiles collected from the original ATCOR results and the cross-track illumination results. The x-profiles were collected from flight line 22004, band 64, line 3483 .

Figure 4.11: Comparison between the ATCOR spectrum and the ELC spectrum for field location SU5.

Figure 4.12: The ELC plot for $1456.5 \mathrm{~nm}$. Each of the points represents one of the four spectra used for the ELC. 152

Figure 4.13: The contribution of the VNIR and SWIR detectors to the image spectra (top). A zoom in on the detector overlap region (bottom) 154

Figure 4.14: A comparison of the AISA data $(6 \mathrm{~m}$; left $)$ and the simulated EnMAP data (30 m; right) (RGB composite; R: $640 \mathrm{~nm}$, G: $550 \mathrm{~nm}$, B: $460 \mathrm{~nm})$.

Figure 4.15: An example of the 'red' vegetation in the simulated EnMAP data (top) (RGB composite; R: $640 \mathrm{~nm}, \mathrm{G}$ : $550 \mathrm{~nm}, \mathrm{~B}: 460 \mathrm{~nm}$ ) and the

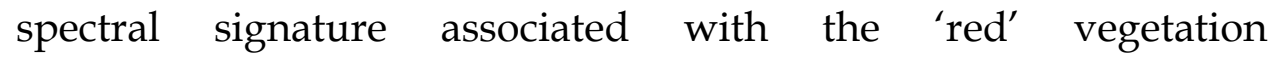
(bottom) .156

Figure 4.16: Spatial distribution of the effect of water vapour over estimation at $940 \mathrm{~nm}$ and $1130 \mathrm{~nm}$ (RGB image on the right; Red $=944 \mathrm{~nm}$, Green $=1040 \mathrm{~nm}$, Blue $=1132 \mathrm{~nm}$ ). Purple areas contain the water vapour overestimation artifacts. Green areas do not exhibit the water vapour overestimation artifact. $R=944 \mathrm{~nm}, \mathrm{G}=1040 \mathrm{~nm}, \mathrm{~B}=1132$ 
$\mathrm{nm}$. The figure on the left shows the maximum, minimum and mean reflectance's of the Region of Interest (ROI) collected from both artifact affected and non-artifact affected areas.............................................................157

Figure 4.17: RMS error image from the AISA unmixing.......................160

Figure 4.18: Histogram of the RMS error values from the AISA unmixing......161

Figure 4.19: Vegetation mask generated from the AISA data. The black pixels compose the mask. All six SU sites (highlighted in red) do not reside under this mask..........................................162

Figure 4.20: RMS error image from the simulated EnMAP unmixing...........164

Figure 4.21: The histogram of RMS error values from the EnMAP

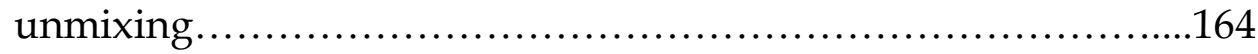

Figure 4.22: Final 30-m AISA soil erosion state validation map. Yellow = accumulated , orange $=$ intermediate and red $=$ eroded $\ldots \ldots \ldots \ldots \ldots . . . .168$

Figure 4.23: Example of unidentifiable spectra, which remained unclassified in the soils unmixing of the EnMAP data 169

Figure 4.24: Elevation profiles from a poorly identified region in the EnMAP unmixing. .170

Figure 4.25: Final EnMAP soil erosion state map. Yellow = accumulated, orange $=$ intermediate and red $=$ eroded. .172

Figure 4.26: Map of the spatial distribution of classification errors overlaid by contour lines. Red = Intermediate classified as Eroded; Green = Accumulation classified as Intermediate; Blue $=$ Eroded classified as intermediate; Yellow $=$ Intermediate classified as Accumulated..........................................................175

Figure 5.1: Comparison of spectra from homogenous land cover that extends over the seam line. Flight line 22003 is referred to as flight line A in the text. Flight line 22004 is referred to as flight line B in the text. 181

Figure 5.2: Shows the required field plot area to allow for a 1 pixel $(6 \mathrm{~m})$ offset. The inner circle represents the $79 \mathrm{~m}^{2}$ plot area used for the 2011 Camarena field work. The outer circle shows the plot area $\left(295 \mathrm{~m}^{2}\right)$ required to allow for a 1 pixel error in the geometric correction. 182 


\section{LIST OF ABBREVIATIONS}

2D- Two Dimensional

3D- Three Dimensional

6S- Second Simulation of the Satellite Signal in the Solar Spectrum

ADC- Analogue to Digital Converter

AISA- Airborne Imaging Spectrometer for Applications

ANSWERS- Areal Nonpoint Source Watershed Environment Response Simulation

AOT- Aerosol Optical Thickness

ARSF- Airborne Research and Survey Facility

ASD- Analytical Spectral Devices

asl- Above Sea Level

ATCOR- Atmospheric and Topographic Correction

AVHRR- Advanced Very High Resolution Radiometer

AVIRIS- Airborne Visible/ Infrared Imaging Spectrometer

BI- Bilinear Interpolation

BOL- Beginning of Life

BRDF- Bidirectional Reflectance Distribution Function

CAI- Cellulose Absorption Index

CAP- Common Agricultural Policy

CC- Cubic Convolution

CoB- Count-based Endmember Selection

CORINE- Coordination of Information on the Environment

CRISM- Compact Reconnaissance Imaging Spectrometer for Mars

CWV- Columnar Water Vapour

DEM- Digital Elevation Model 
DN- Digital Number

EAR- Endmember Average RMSE

EeteS- EnMAP end-to-end Simulation software

EFFORT- Empirical Flat Field Optimized Reflectance Transformation

ELC- Empirical Line Correction

EnMAP- Environmental Mapping and Analysis Program

ENVI- Environmental Visualization Software

EO- Earth Observation

EOL- End of Life

ETM+- Enhance Thematic Mapper Plus

EU- European Union

EUROSEM- European Soil Erosion Model

FAO- Food and Agriculture Organization

FFT- Fast Fourier Transformation

FOV- Field of View

FWHM- Full Width Half Maximum

GAEC- Good Agriculture and Environmental Conditions

GCP- Ground Control Point

GDP- Gross Domestic Product

GFZ- Helmholtz Centre Potsdam, German Research Centre for Geoscience

GMT- Greenwich Mean Time

GPS- Global Positioning System

GSD- Ground Sampling Distance

HyMAP- Hyperspectral Mapper

IDW- Inverse Distance Weighting

IFOV- Instantaneous Field of View 
IMU- Inertial Measurement Unit

ITC- Faculty of Geo-Information Science and Earth Observation

L-1- Level-1 Detector images coregistered

L-2- Level-2 (L-2atm and L-2geo)

L-2atm- Level-2 Atmospherically Corrected

L-2geo- Level-2 Geometrically Corrected

LiDAR- Light Detection and Ranging

MASA- Minimum Average Spectral Angle

MESMA- Multiple Endmember Spectral Mixture Analysis

MNF- Minimum Noise Fraction

MODTRAN- Moderate Resolution Atmospheric Transmission

MSS- MultiSpectral Scanner

MTMF- Mixture Tuned Matched Filtering

N-D- N-Dimensional

NDVI- Normalized Difference Vegetation Index

NERC- National Environmental Research Council

NGO- Non-Government Organization

NIR-Near Infra-Red

NN- Nearest Neighbour

NOAA- National Oceanic and Atmospheric Administration

NRCAN- Natural Resources Canada

PARGE- Parametric Geocoding and Orthorectification

PCA- Principal Component Analysis

PICASSO- Parameterized Image Chain Analysis and Simulation Software

PPI- Pixel Purity Index

PSF- Point Spread Function 
RADAR- Radio Detection and Ranging

RENDVI- Red Edge Normalized Difference Vegetation Index

RGB- Red, Green, Blue

RMS- Root Mean Square

RMSE- Root Mean Square Error

ROI- Region of Interest

ROME- Reduction of Miscalibration Effects

RT- Radiative Transfer

SAM- Spectral Angle Mapper

SAR- Synthetic Aperture RADAR

SEDMEDHY- Soil Erosion Detection within MEDiterranean agricultural areas using Hyperspectral data

SEMMED- Soil Erosion Model for Mediterranean Regions

SENSOR-Software ENvironment for the Simulation of Optical Remote sensing systems

SMA- Spectral Mixture Analysis

SMACC-Sequential Maximum Angle Convex Cone

SNR- Signal to Noise Ratio

SPECTRA- Surface Processes and Ecosystem Change Through Response Analysis

SPOT-Satellite Pour l'Observation de la Terre

SRF- Spectral Response Function

SSI- Spectral Sampling Interval

SU- Sothern Field Site

SWIR- Short Wave Infra-Red

TM- Thematic Mapper

TOA- Top of Atmosphere 
UNCCD- United Nations Convention to Combat Desertification

UNEP- United Nations Environment Programme

USLE- Universal Soil Loss Equation

VI- Vegetation Index

VIR- Visible/Infra-Red

VIS- Visible

VNIR- Visible Near Infra-Red

WATEM- Water and Tillage Erosion Model

WEPP- Water Erosion Prediction Project 


\section{Introduction}

Soil, next to water, is the most vital resource for the continued prosperity of human populations (Morvan et al.,2008; Yang et al.,2003; Doran, 2002). The primary threat to the continued productivity of the soil resource is the mismanagement of it (Boellstroff and Benito, 2005; Herrick, 2000; MartinezCasaanovas and Sanchez-Bosch, 2000), for example, by employing unsustainable farming practices. Regular monitoring of the soil resource is necessary for the early identification of degradation, which would allow ample time for the implementation of countermeasures.

It is estimated that 77 billion tons of soil is lost each year to soil erosion at an estimated cost of $\$ 400$ billion annually (Eswaran et al., 2001). It is also estimated that nearly one third of the world's arable land has been lost to erosion in the last 40 years (Yang et al., 2003). The loss of arable land has devastating effects on both food production and sustainability (Pimentel, 2006). Soil erosion effectively diminishes soil quality, which in turn has devastating effects on the productivity of not only agricultural ecosystems but also forest and rangeland ecosystems (Pimentel and Kounang, 1998; Pimentel, 2001).

There are various physical, chemical and biological mechanisms that contribute to overall land degradation (Lal, 1994). Soil erosion is among the many physical mechanisms and is considered highly indicative of severe stages of land degradation. There are four primary drivers of soil erosion (Lal, 2001). 
These drivers are physical by means of wind and water, chemical, gravitational and agricultural such as tillage. Soil erosion in semi-arid Mediterranean environments, like those found in many regions of Spain, are closely related to geographical and geological factors, such as lithology, topography and climatology, as well as extensive anthropogenic activities. Among the many mechanisms influencing and affecting soil erosion in Spain are anthropogenic impacts such as deforestation and intensive agricultural practices (Garcia-Ruiz, 2010).

Spain has been subject to major changes in land use and land cover over the last several decades, resulting in increasing susceptibility to soil erosion (Symeonakis et al., 2007). Various biophysical, socio-economic and political changes have greatly influenced the intensity of soil erosion in the semi-arid Mediterranean region of Spain (Symeonakis et al., 2007). Biophysical activities include forest fires, agricultural intensification and tillage practices (Govers 1994; Lindstrom, 1992 and 1990). Socioeconomic and political examples include relocation of the human population to coastal areas, the rapid expansion of tourism and the European Common Agricultural Policy (CAP) set-aside policy and financial incentives (Garcia-Ruiz, 2010; Boellstorff and Benito, 2005).

\subsection{Remote Sensing of Soil Erosion}

Remote sensing of soils was established with the use of aerial photography. The known relationship between the patterns of the soils and the 
terrain (e.g., relief, drainage conditions, vegetation, etc.) provided the necessary information for the analyst to build a soils inventory (FAO, 1967). Digital soil mapping emerged as a reliable soil mapping alternative when the availability of both digital spatial data (e.g., Digital Elevation Models and Landsat) and computing power increased (Minasny and McBratney, 2014).

Hyperspectral image analysis is a more recent tool that has proven itself valuable for mapping and monitoring soil erosion processes (Ben-Dor et al., 2009; Shrestha et al., 2005). Hyperspectral data introduced narrow, contiguous spectral bands that made it possible to resolve the very narrow absorption features of soil spectra (Escribano et al., 2010).

Hyperspectral remote sensing identifies the presence of eroded soils based on differences in spectral reflectance. These differences in spectral reflectance can be seen on the surface as alterations in the physical state of the soil for example increased stoniness or lack of organic matter. Other physical soil states include salinity or alkalinity of low-lying areas due to poor infiltration to deeper soil horizons, crusting and the exposure of mineral horizons at the surface (Shrestha et al., 2005; Lal, 2001).

Traditional methods of monitoring land degradation through field observations are time consuming and expensive (Tromp and Epema, 1999). Remote sensing methods have proven to be more economical while providing reliable information. Multispectral sensor systems such as Landsat Enhance 
Thematic Mapper Plus (ETM+), Satellites Pour lèObservation de la Terre (SPOT) and Advanced Very High Resolution Radiometer (AVHRR), have successfully been applied to soil mapping (Cole and Boettinger, 2007; Barnes and Baker, 2000; Odeh and McBratney, 2000). The advent of operational space-borne hyperspectral remote sensing systems promises to enhance the ability of remote sensing to retrieve key biophysical and biochemical soil variables. These systems also provide frequent revisit times (e.g., 3-4 days for the Environmental Mapping and Analysis Program (EnMAP; Kaufmann et al., 2012)) which allows for the continual monitoring of the environmental conditions at a specific site.

For future monitoring of soil erosion and other land degradation processes, it is important to assess the capability of upcoming hyperspectral Earth Observation (EO) systems for detecting and mapping degradational features. It can contribute in this assessment. Scene simulation software is important for not only the design of new EO systems, but it also allows for the understanding of the effects of different instrument and environmental parameters on image characteristics, aids in the development and validation of data processing algorithms and can also assists in the investigation of possible scaling issues (Segl et al., 2012; Cota et al., 2010; Parente et al., 2010; Peisker et al., 2010).

Within the scope of the EnMAP mission, the extraction of information on and the monitoring of sediment properties, soil erosion status and land 
degradation are core priorities (Stuffler et al., 2008). Simulation software, such as the EnMAP end-to-end Simulation (EeteS) tool, can be used to produce EnMAP reflectance image cubes, which in turn can be used to investigate the capabilities of the future EnMAP satellite data for soil erosion and land degradation applications and to act as a test bench for similar scientific exploration using the EnMAP system (Segl et al., 2012). Simulation studies, as presented in this research, are essential for understanding how future satellite image products will be useful in the future.

The availability of spaceborne hyperspectral sensors, such as EnMAP, will allow for frequent and effective collection of hyperspectral image data. Increased access to hyperspectral image data will provide a basis for the generation of more effective soil erosion detection methods as well as possibly act as a starting point for procedural and product standardization in remote sensing soil erosion investigations.

This research utilized the Airborne Imaging Spectrometer for Applications (AISA) data collected from the Soil Erosion Detection within MEDiterranean agricultural areas using Hyperspectral data (SEDMEDHY) test site located in central Spain to simulate an EnMAP reflectance image cube. The simulated image cube was then used to investigate the mapping capabilities of the future EnMAP satellite in semi-arid Mediterranean soil erosion applications. 


\subsection{Objectives}

The main objectives pursued in this research were:

1. Discriminate bare soil, crop residue and sparse vegetation in simulated EnMAP hyperspectral data using image analysis.

2. Effectively map soil erosion states using hyperspectral decomposition techniques.

The following chapters will provide an overview of relevant existing literature, outline the procedures and methods employed in this research and present the results obtained. The work will be concluded with an in depth discussion of the procedures leading to the results as well as of the results themselves. 


\section{Literature Review}

\subsection{Soil-Introduction}

Soil formation is described as the transformation of rock, or parent material, into soil (Jenny, 1941). Simonson (1959) describes the process of soil formation as consisting of two processes: 1) the accumulation of parent material, and 2) the development of soil horizons. Although the processes may occur at different rates and in different sequences in various regions, they are consistent on a global scale. Compositionally, all soils are a combination of mineral material, organic material, water and air. However, the proportions and characteristics of each component may vary by region (Cambardella et al., 1994; Robertson et al., 1993; Simonson, 1959) as a result of the spatial variation of soil forming factors ( e.g., parent material, climate, topography and organisms; Jenny, 1941).

Soils support the planets terrestrial vegetation and contains a significant portion of the world's carbon as organic matter (Nature Geoscience, 2010). All soils also play an important role in ecosystem wellbeing, namely through partitioning rainfall, maintaining habitat diversity and stability, buffering against toxins, and storing, recycling and partitioning nutrients and energy (Warkentin, 1995). In addition to the atmospheric, hydrospheric and lithospheric functions of soils, they also serve as an essential component of any functioning ecosystem (Lal, 2013; Doran and Parkin, 1994). 


\subsubsection{Land Degradation}

Land degradation is a process, which decreases the capacity of the soil to perform its atmospheric, hydrospheric and lithospheric functions, as well as threatens food production and other ecosystem goods and services (Stewart and Lal, 1992). It is largely a human-induced process (UNEP, 1988), with contributing effects from natural phenomena, such as floods, drought and landslides (UNEP, 2013). Human activities such as unsustainable agricultural practices, poor soil and water management practices and vegetation removal by means of deforestation and burning, all contribute to the degradation of the soil (UNEP, 2013). Included in this is the expansion of almond and olive orchards into marginal lands which are comprised mainly of steep, stony hill slopes (GarciaRuiz, 2010). More so than water erosion, it is tillage erosion which is the main cause of land degradation in these marginal lands (Poesen et al., 1997).

In some instances this has resulted in mass movement of and, in extreme cases, the complete destruction of civilizations, as occurred with the ancient civilizations of Mesopotamia and the Indus Valley (Hillel, 1991; Adams, 1981).

In arid, semi-arid and dry sub-humid regions, land degradation is also referred to as desertification (UN, 1994; Stewart et al., 1991) and can lead to permanent loss of land productivity (Wang et al., 2006;Yang et al. 2005). However, in some instances the productivity of the land can be reclaimed, depending on variables, such as the degree of degradation, continued human 
involvement and climatic factors (Toky and Ramakrishnan, 1981; Bazzaz, 1968). Maintaining and managing land productivity will be essential in the years to come in regards to the issue of global population growth and the growing pressures on farmlands to increase food and/or livestock outputs (Gilland, 2002).

\subsubsection{Soil Erosion}

The natural soil erosion process has been occurring since the development of the first soils, which occurred approximately 450 million years ago (FavisMortlock, 2007). Soil erosion is one of many natural processes that contributes to global land degradation. It is defined as the detachment and transport of soil particles by wind or water (Favis-Mortlock, 2007; Poesen and Hooke, 1997), but can also be influenced by such things as tillage and mass movement (Poesen and Hooke, 1997). Soil erosion is a completely natural process; however, the rate of soil erosion poses an environmental issue (Favis-Mortlock, 2007). Both natural and anthropogenic influences determine the rate of soil erosion (Lal, 2001).

Broad examples of natural factors that contribute to soil erosion include climate, vegetation and topography (Lal, 2001). More specifically the drop size distribution and intensity of rain and the slope gradient, length, aspect and shape of the terrain (Lal, 2001). The threat to the Earth's soil resources by natural erosion processes is minimal since the rate of soil erosion is often equivalent to the rate of soil formation (Favis-Mortlock, 2007). 
This is not the case with accelerated soil erosion processes. Anthropogenic factors, such as land-use, farming practices and soil management (Lal, 2001), accelerate the soil erosion process, which results in the removal of soil at a rate that is much faster than it can be replenished (Favis-Mortlock, 2007). The earliest accounts of accelerated soil erosion are associated with the implementation of early agriculture practices (Favis-Mortlock, 2007). In addition to the direct anthropogenic factors influencing soil erosion, there are also socio-economic factors that influence soil erosion such as poverty and rapid population growth (Lal, 2001; Richards, 1990).

There are several countries in the world that are affected by accelerated soil degradation. These include, the Sahelian and Chinese arid and semi-arid regions, followed by the Iranian and Middle Eastern drylands (UNEP, 2012). Common to each of these areas is a high-spatial and temporal variability in rainfall and intensive grazing (UNEP, 2012), which both largely affect vegetation cover.

At a local level, soil erosion can reduce the productivity of the soil, alter plant composition and negatively impact biodiversity, both above and within the soil (Pimentel et al., 1995). The far reaching effects of soil erosion can be devastating, particularly when the soil particles enter the water systems (USDA, 1990). Once this happens, the negative effects can reach regional scales (FavisMortlock, 2007). These off-site disturbances include eutrophication of water 
bodies, siltation of harbours, loss of reservoir storage, flooding, disruption of stream ecology and loss of wildlife habitat (Lal, 1998; Gray and Leiser, 1982).

\subsubsection{Soil Erosion in Semi-Arid Mediterranean Environments}

Semi-arid Mediterranean environments are characterized by warm to hot, dry summers with mild to cool winters and relatively low annual rainfall (between $25 \mathrm{~cm}-50 \mathrm{~cm}$ ). The rainfall is concentrated mainly in the winter months, with the heaviest rainfall occurring in November. Globally, arid and semi-arid environments account for $40 \%$ of the land surface (Deichmann and Eklundh, 1991) and contain approximately $38 \%$ of the global population (Reynolds et al., 2007; Veron et al., 2006).

Soil erosion rates in Mediterranean arid and semi-arid environments range between $0.4 \mathrm{~mm}$ to $1.7 \mathrm{~mm}$ annually (Benito et al., 1992). Erosion by water is the largest natural form of soil erosion found in Mediterranean arid and semiarid environments (Poesen and Hooke, 1997; Schlesinger et al., 1990). Erosion by wind is uncommon and is only known to occur on costal sandy soils and as a result of tillage, which produces local dust clouds (Poesen and Hooke, 1997).

The Mediterranean has a long and spatially expansive history of soil erosion and land degradation (Butzer, 2005). Both natural and anthropogenic factors play a role in the Mediterranean's high susceptibility to soil erosion. High rainfall intensity, low average annual precipitation, fragile soils with low organic matter content and poor nutrient content, steep slopes, removal of vegetation 
resulting from deforestation and mining, and land-use changes have all contributed to an unstable soil situation (Grove and Rackham, 2003; Thornes and Wainwright, 2003; Kosmas et al., 2000; Poesen and Hooke, 1997).

Natural soil erosion processes commonly found in semi-arid Mediterranean environments include rain splash erosion and rill and gully erosion. Although these erosion types are all forms of water erosion, the consequences of each are vary greatly (Favis-Mortlock, 2007).

Rain splash erosion is common in these types of environments because of the typical heavy rainfall events, sloped surfaces and lack of vegetation cover. Similarly, the same environmental characteristics also play a dominant role in rill and gully erosion. However, the areal effects of this type of erosion extend much further than that of rain splash erosion (Favis-Mortlock, 2007).

The effects of the latter remains quite localized, whereas rill and gully erosion can affect entire watersheds if the sediments end up being deposited in adjacent water systems (Favis-Mortlock, 2007). In many cases, this can be quite devastating to the surrounding ecosystem because the erosion debris is often known to clog parts of streams and silt up reservoirs, resulting in higher magnitude and frequency flooding (Poesen and Hooke, 1997; Bennett, 1960).

In Mediterranean environments, vegetation cover is one of the main determinants controlling the extent of soil erosion (Lacaze, 1996). To significantly 
reduce soil erosion in semi-arid environments, a plant cover exceeding $60 \%$ is required (Sauer and Ries, 2008). Accordingly, soil erosion develops in areas where the vegetation has been seriously damaged or drastically altered over a short period of time (Hill et al., 1995; Francis and Thornes, 1990).

Agriculture has been a prominent land-use in the Mediterranean for centuries (Stevenson and Harrison, 1992) and has had adverse impacts. Accordingly, past and present soil erosion issues in Spain are strongly tied to agricultural activities (Ortega and Simo, 2007). In Spain, the main agricultural land-uses are vineyards, olive groves and rain-fed cereal crops (Garcia-Ruiz, 2010).

These agricultural land-uses are highly susceptible to erosion. Vineyards and olive groves are often cultivated on steep slopes and even in peek growing season, a large amount of soil in between rows is exposed leaving not only sufficient area for water erosion, but also influencing the direction of the soil transport (Garcia-Ruiz, 2010; De Graaf and Eppink, 1999; Garcia-Ruiz et al., 1995). In contrast, rain-fed cereals, although providing sufficient soil cover in the growing season, are often cycled through years of fallow, which leave the entire surface exposed for long periods of time (Garcia-Ruiz, 2010).

It is not only the type of cultivation that influences soil erosion in Spain, but also the rapid land-use change associated with agriculture and the agricultural practices employed. Rapid land-use change, particularly in semi-arid 
environments, is devastating to soil health because it is often associated with the destruction of natural vegetation cover, which protects the soil from raindrop impact (Pugnaire et al., 2006; Dunjo et al., 2003).

Historically, rapid land-use change in Spain has been associated with the expansion of agriculture to meet international demands, such as the increasing American demand for wheat and wood in the 19th century, and to evade financial hardships (Puigdefabregas and Mendizabal, 1998; Darby, 1956). More recently, rapid land-use change has been associated with farmland abandonment, resulting from the depopulation of rural areas, the inability to use modern machines on the land and issues regarding soil fertility (Garcia-Ruiz, 2010; Kosmas et al., 2000; Puigdefabregas and Mendizabal, 1998). Farmland abandonment is particularly devastating in semi-arid environments because of the scarcity of rainfall which makes plant colonization difficult (Pugnaire et al., 2006). However, the addition of fertilizers at the time of abandonment has been proven to encourage plant colonization which, would decrease the risk of soil erosion associated with farmland abandonment (Lasanta et al., 2000). Other agricultural practices that have had a devastating effect on soil erosion are those associated mainly with the intensification of agriculture, such as irrigation, pesticides and most importantly, tillage (Aidoud et al., 1998).

Currently, agriculture provides 2.8 \% of Spain's Gross Domestic Product (GDP) and accounts for $12.7 \%$ of the total European agriculture sector (European Commission, 2012a). Maintaining healthy soils is mandatory if production is to 
be sustained at current levels. However, the low-risk perception farmers have towards agriculturally induced soil erosion may interfere with the introduction and implementation of soil erosion control measures (Ortega and Simo, 2007).

In the province of Castilla-La Mancha, a recent survey revealed that farmer's had a low risk perception of erosion control factors, such as framing on sloped areas, minimum soil cover and maintenance of terraces (Ortega and Simo, 2007). It also revealed that the compliance measures put in place to help mitigate soil erosion processes are not strictly followed and that the farmers low risk perceptions correlated to a perceived lack of information. There was also a similar correlation in the data regarding farmer's perceptions concerning water use. In contrast, the Good Agricultural and Environmental Conditions (GAEC; Ortega and Simo, 2007) involving water use had a high risk perception, high level of information provided and high level of compliance. These correlations illustrate that, with high levels of information, there is the potential for improved soil erosion risk perception and a higher levels of compliance.

Tillage practices, in particular, should be perceived as high risk in terms of soil erosion impact. The effects of tillage on soil erosion can be both indirect and direct. The indirect effect of tillage is related to the disruption of key soil characteristics, such as reduction of soil organic matter, decrease in aggregation and stability of the aggregates, disruption of macropores, as well as negative impacts on soil biodiversity and macrofauna (Lal, 1993). By drastically altering 
these characteristics, the soil is left more vulnerable to erosive processes and becomes more susceptible to compaction and erosion by wind and water, particularly rain drop impact (Lal, 1993). Deterministic erosion models such as the Water Erosion Prediction Project (WEPP) Model and the European Soil Erosion Model (EUROSEM) try to account for the indirect effects of tillage practices on soil erosion (Morgan et al, 1992; Nearing et al., 1989).

The severity of the erosion related directly to tillage is highly dependent on topography. It has been implied by Govers et al. (1994) that within the hilly landscapes of Western Europe, sedimentation rates associated directly with tillage were greater than those associated with water erosion. Hilly landscapes are so drastically impacted by tillage-induced soil erosion, because the movement of soil, as a result of tillage, is directly related to the slope (Lindstrom et al., 1990). As the tillage alternates upslope and downslope, a greater amount of soil was observed to move downslope than upslope, resulting in a net downward motion (Govers et al., 1994; Lindstrom et al., 1990). In this case, the soil traveled from the convex slope positions and deposit in the concave slope positions (Lindstrom et al., 1992).

The main property of the slope that influences the movement of soil by tillage is changes in the slope gradient (Govers et al., 1994). This means that the accumulation of soil can occur along the side of a hillslope as long as the difference in slope between two adjacent positions is the same. The Water and 
Tillage Erosion Model (WATEM; Oost et al., 2000) is capable of assessing the effects of landscape type and its change on tillage and water induced soil redistribution at a watershed scale. Considering both water and tillage erosion simultaneously is beneficial, because each erosional process is influenced by different topographical characteristics, and the effects can often become compounded at a larger watershed scale (Oost et al., 2000).

\subsubsection{European Common Agricultural Policy- Set-Aside Policy}

Europe is the second largest food exporter and the largest food importer in the world (European Commission, 2012a), and thus it is crucial that the productivity of the land be maintained. The Second World War resulted in the destruction of large areas of European agricultural land. To help remediate the damage to the land, the European Economic Community (a precursor of the European Union (EU)) implemented the CAP in the 1950's (European

Commission, 2012b). It was initially implemented in Western Europe and focused on encouraging better agricultural productivity through subsidies and higher prices for goods to promote greater production (European Commission, 2012a).

Since the 1950's, the CAP has expanded and evolved to accommodate changing agricultural needs and marketplace demands. The CAP that people are familiar with today was developed largely in the 1980 's to early 1990 's and was chiefly influenced by the MacSharry reform (European Commission, 2014). The 
changes that the reform brought about focused mainly on marketplace stabilization and environmental protection.

The most drastic change relating to the marketplace was the shift from product support, through guaranteed prices, to producer support, through income compensation. This was done primarily to curb the surplus and stabilize the agricultural markets. There was also a shift to increase the competitiveness between EU farmers and diversify production (European Commission, 2014).

Many incentives and measures were put in place with the MacSharry reform to help with environmental protection. Incentives were provided for the adaption of agricultural practices to support environmental sustainability, such as reducing inputs into the land, such as fertilizers and pesticides, leaving field boundaries uncultivated and introducing landscape features that increase diversity and reduce erodibility (European Commission, 2012a).

Other agro-environmental measures that have been implemented include afforestation, early retirement, diversification and compulsory set-aside. The setaside in particular, requires some of the land to be taken out of production if a certain output threshold is exceeded (European Commission, 2002). The anticipated result of the set-aside policy included curbing production and reducing surplus, preserving small farmers and maintaining the environment (European Commission, 2002). 
The effectiveness of the set-aside policy on environmental protection, particularly soil erosion, varies substantially on a regional basis, because the policy does not consider or allow for flexibility to account for the varying climatic, environmental and economic conditions of the different agricultural regions throughout Europe (Boellstorff and Benito, 2005). In many countries soil protection has been effectively incorporated into the set-aside policy through mandatory cover of the set-aside land. However, this was done under the national laws of individual countries.

Unfortunately, Spain has not established any such law in order to control soil erosion on set-aside land (European Commission, 2002). In Spain, two forms of set-aside can be implemented, the first being unseeded fallow and the second being seeded fallow (Boellstorff and Benito, 2005). The majority of Central Spain uses unseeded fallow and the percentage of arable land kept under unseeded fallow between the years of 1992, when the set-aside was put into effect, and 1995 increased by $7 \%$ (Boellstorff and Benito, 2005). Unseeded fallow is frequently chosen, because it increases soil moisture, which is a limiting factor on crop yields especially in arid and semi-arid regions. However, this type of fallow is highly susceptible to soil erosion. Boellstorff and Benito (2005) revealed that the soil loss rate of seeded fallow is $50 \%$ less than that of unseeded fallow. Boellstorff and Benito (2005) also concluded that, due to the economic incentives offered, farmers put greater percentages of their land into set-aside. The rationale 
is that the economic incentives are more favorable than the income from the crop yields as a result of the lower land quality. The lack of a formal and enforceable soil protection policy in Spain has created a situation that promotes agricultural practices that hinder soil protection and conservation.

\subsubsection{Soil Erosion Assessment/Monitoring/Management}

The assessment, monitoring and management of soil erosion are strongly interconnected. Assessment allows for an understanding of the current state of the soil and, when the assessment is conducted for a location more than once over a period of time, it results in the monitoring of the soil condition overtime. Both the assessment and monitoring of the soil condition can then be incorporated into establishing an appropriate management scheme for a specific location.

\section{Assessment}

Many methods exist for the assessment of soil condition. Examples of such methods include the employment of expertise knowledge, factor scoring and modeling (van der Knijff et al., 2000; Morgan, 1995). The Soil Erosion Risk Map of Western Europe by De Ploey (1989) is an example of a product generated using individuals' expertise, by which experts in a location use their extensive knowledge to delineate erosion risk locations (van der Knijff et al., 2000). 
Factor scoring is a method that has been employed by both Coordination of Information on the Environment (CORINE) for their soil erosion risk assessment of the Mediterranean region (Briggs, 1992) and the Food and Agriculture Organization ( FAO)/ United Nations Environment Programme (UNEP) for their assessment of desertification (Dregne and Boyadgiev, 1983). Unfortunately both methods, although providing an assessment of the soil condition, come with their limitations.

The Soil Erosion Risk Map of Western Europe provides no explanations of the methodology or criteria used to evaluate soil erosion (Yassoglou et al., 1998). Without providing a stable definition of the characteristics and criteria used to evaluate soil erosion the interpretation of the Soil Erosion Risk Map of Western Europe is left to each individual interpreter. This is similar to factor scoring which, although providing very well defined qualitative erosion classes, the interpretation of these classes are often subjective and can sometimes be quite difficult to interpret (van der Knijff et al., 2000).

Models provide an advantage over the aforementioned soil assessment methods, because they are able to assess risk over large areas without the need for extensive ground survey (van der Knijff et al., 2000). Models used to assess soil erosion and soil erosion risk are designed based on a range of temporal and spatial scales, as well as empirically or physically based model types and investigation of different types of erosion (van der Knijff et al., 2000). Examples 
of erosion models include Areal Nonpoint Source Watershed Environment Response Simulation (ANSWERS; Beasley et al., 1980), WEPP (Nearing et al., 1989), the Universal Soil Loss Equation (USLE; Jager and Rickson, 1994) and the Soil Erosion Model for Mediterranean Regions (SEMMED; Morgan, 1984; De Jong, 1994).

Certain limitations also exist when using models to assess soil erosion. Firstly, uncertainties of any type, for instance in the input data and definable variables or model requirements, can propagate as errors through the entire modelling process and have a negative impact on the end result (van der Knijff et al., 2000). Although it is understood that the purpose of a model is not to provide a perfect solution to any one problem, these uncertainties still influence the final product and should be kept in mind. Secondly, the application of models in soil erosion assessment are further complicated by the lack of field validation, which is a difficult and often disregarded aspect of the modelling process (van der Knijff et al., 2000).

Currently, there is no consensus on the correct way to assess desertification (Veron et al., 2006). The United Nations Convention to Combat Desertification (UNCCD, 2000) has also indicated that although there is a large amount of data available, gathering a clear understanding of land degradation at regional or national levels has not been possible. The main reason for this is a 
result of inconsistent assessment methods and excessive subjectivity, producing different estimates of soil erosion (Veron et al., 2006; Agnew and Warren, 1993).

\section{Monitoring}

The monitoring of soil erosion consists in providing an assessment over time. The main issues to overcome to make soil erosion monitoring more applicable to management purposes, are providing information at appropriate scales and maintaining a standardization of methods and techniques between studies. Not only are soil surveys at meaningful temporal and spatial scales rare (Lacaze, 1996), there is also a lack of sufficient reference information about the initial condition of the landscape to which desertification, or soil degradation in general, can be compared (Veron et al., 2006). A situation such as this has been observed in southeastern Spain by Boer and Puigdefabregas (2003).

The temporal scale of soil erosion monitoring depends on the type of erosion being monitored. Erosion over longer time periods can be caused by wind-blown events, whereas a large rain storm can cause extensive erosion in a short time period (Favis-Mortlock, 2007). Spatial scales of soil erosion are also variable, ranging from a localized scale to affecting an entire catchment area (Favis-Mortlock, 2007; Poesen and Hooke, 1997). Accordingly, both the spatial and temporal scales of the monitoring need to be suited to the type of erosion occurring in order for any meaningful results to be derived. 
Similarly to how a lack of standardization in soil assessment limits the compatibility of data over space, a lack of standardization in the methods and techniques used for soil monitoring are also a limiting factor in that they restrict the compatibility of different data sources over time (Lal, 2001).

\section{Management}

Methods of soil management and conservation currently used in agriculture are generally short-term strategies, such as fertilizers and irrigation. To effectively combat soil degradation, long-term management strategies need to be implemented. However, this is often difficult because long-term strategies are difficult to quantify and will only make a visible impact after several decades (Herrick, 2000). Methods such as biomass mulches, crop rotations, no-till and added grass strips, all support soil conservation by protecting the soil from wind and rain erosion (Pimentel et al., 1995; Pimentel, 1993; Troeh et al., 1991).

Truly managing soil degradation is going to take much more than simply understanding the processes of soil erosion and reciting the necessity of conservation. It will require an understanding of human-environment interactions (Reed et al., 2011). To gain a comprehensive insight into the impact of human-environment interactions on soil erosion, both the biophysical and socio-economic realms of the soil degradation process will need to be investigated simultaneously (Reed et al., 2011; Reynolds et al., 2007). In addition to the human-environment interactions, society will need to begin to view soil as 
an essential and irreplaceable natural resource (Doran and Zeiss, 2000;

Warkentin, 1995). This will not only involve making information accessible to the masses, but also making it easily understandable and the solutions easily applicable (Weiskel, 1989).

Successfully combating soil degradation and desertification will not only require the involvement of the government, businesses, Non-Government Organizations (NGO's) and international organizations (Yang and $\mathrm{Wu}, 2010)$, it will also demand involvement of local communities and greater acceptance and utilization of local environmental knowledge (Reed et al., 2011; Reynolds et al., 2007).

\subsection{Remote Sensing}

Remote sensing is defined by Colwell et al. (1983) as, the measurement or acquisition of information of some property of an object or phenomenon, by a recording device that is not in physical contact with the object or phenomena under study. In the scope of earth sciences, remote sensing is focused towards the collection of information regarding the Earth's surface and its constituents. Gathering information about the Earth's physical, chemical and biological systems using remote sensing techniques is referred to as Earth Observation (EO; NRCAN, 2013).

A series of sensor types can be used for EO. The two broad categories of sensors used for EO are active and passive sensors. Active sensors produce and 
receive their own signal, whereas passive sensors record electromagnetic radiation reflected or emitted from the scene (Shippert, 2002). Examples of active sensors include Light Detection and Ranging (LiDAR) and Radio Detection and Ranging (RADAR). Passive sensors include aerial photography, multispectral sensing and hyperspectral sensing. Remotely sensed images can be described in terms of a series of characteristic resolutions. The four main resolutions are radiometric resolution, spatial resolution, spectral resolution and temporal resolution.

The radiometric resolution of a sensor describes the detectors sensitivity to the magnitude of the electromagnetic energy (NRCAN, 2013a). A sensor with low radiometric resolution will only be able to detect large differences in the magnitude of the radiant flux. This will result in less contrast within the scene and, thus, a loss of information.

Spatial resolution is a measure of the smallest angular or linear separation between two objects that can be resolved by the remote sensing system (Jensen, 2005). The pixel size is the smallest unit of an image and is typically used to describe the spatial resolution. Another method to portray the spatial resolution of a sensor is the Ground Sampling Distance (GSD), which is the distance between pixel centers on the ground. The spatial resolution of a satellite sensor is fixed by the Instantaneous Field-of-View (IFOV) and the altitude. However, the 
spatial resolution of an airborne sensor can vary because the altitude is variable (Atkinson, 1997; Barnsley and Curran, 1990).

The spectral resolution of a sensor describes its ability to delineate wavelength intervals (NRCAN, 2013b). Spectral resolution is defined by the number and dimension (eg. position or width) of the specific wavelength regions that the sensor is sensitive to (Jensen, 2005). These specific wavelength regions are referred to as spectral bands.

Finally, temporal resolution describes how often a remote sensing system records data from a particular area. Temporal resolution is controlled by the sensor capabilities. For example, airborne imagery can collect image data when from a location whenever it is desired, however airborne flights require preparation and can be financially exhaustive. On the other hand, satellite sensors are continually collecting data but collection from a certain location is limited by the satellite revisit time, off-nadir pointing capabilities and environmental factors such as cloud cover.

Although these resolutions are used to describe the image data, there are many other sensor components and characteristics, such as the Signal-to-Noise Ratio (SNR), that contribute to the final image product. It is the trade-off between these resolutions and the other sensor characteristics that defines the capabilities and limitations of each type of sensor (Goetz et al., 1985). 


\subsubsection{Hyperspectral Data}

Hyperspectral remote sensing or imaging spectroscopy is defined by Goetz (1985) as the acquisition of images in hundreds of narrow contiguous, spectral bands such that for each pixel a radiance spectrum can be derived. Conversely, multispectral sensors record in a limited number of broad spectral bands (e.g., Landsat 8 - Band 4 (red), $300 \mathrm{~nm}$ band width).

The shift from multispectral sensors (e.g., Landsat 7 ETM+), to hyperspectral sensors (e.g., Hyperion, Airborne Visible/ Infrared Imaging Spectrometer (AVIRIS)) has allowed for image analysis to move from merely identifying what surface cover is present within a pixel, to having the ability to estimate what proportion of the surface cover (fractional cover) is there and derive information about biophysical and biochemical characteristics. Techniques such as spectral unmixing are often employed in hyperspectral data analysis (Malenovský et al., 2007; Shrestha et al., 2005; Clark, 1999). The ability to identify fractional cover within a pixel is a valued capability of hyperspectral remote sensing because most pixels include several surface materials (Malonorvsky et al., 2007; Shippert, 2002).

Many applications, such as soil studies, are beginning to explore the vast benefits available through hyperspectral remote sensing image analysis. Hyperspectral data greatly increases the accuracy of soil mapping in comparison to multispectral sensors (Clark et al., 1990; Kruse, 1989), because broad-band 
sensors cannot provide the spectral detail that is required to detect the high variability exhibited by soils (Deventer van et al., 1997; De Jong, 1994; Irons et al., 1989).

Because most techniques applied to image data of broad-band sensors are not ideal for use with hyperspectral sensors, there is a lack of availability of automatic and easy to use toolboxes applicable to hyperspectral data (Chabrillat et al., 2011; Cloutis, 1996). This, along with the lack of an operational hyperspectral mission, has been a great limitation to the expansion of the use of hyperspectral imagery (Chabrillat et al., 2011).

\subsubsection{LiDAR}

For the collection of terrain data, LiDAR is considered the most efficient and reliable sensor available due to its high geometric accuracy, high sampling density and active illumination (Liu, 2008; Raber et al., 2007; Forlani and Nardinocchi, 2007). Analysis of terrain data provides information on certain landform characteristics that can later be used for soil prediction (Odeh et al., 1993). This is especially useful in soil erosion investigations because a strong predictive relationship has been identified between environmental variables, particularly derived from Digital Elevation Models (DEM)'s (Wilson and Gallant, 2000), and soil properties (Gessler et al., 1995; Odeh et al., 1993).

Most LiDAR systems used for EO are airborne LiDAR systems, the alternative being ground based LiDAR systems and some satellite LiDAR 
systems. The LiDAR system works by sending out a series of rapid laser pulses towards the surface and measures the time it takes for each of the laser pulses to reflect back. The measured time duration can then be used to calculate the distance the laser pulse traveled, effectively giving a series of elevation measures (e.g., from bare earth or canopy height).

The collective set of data points retrieved by a LiDAR system is called a point cloud. The point cloud is essentially made up of a series of three dimensional spatial coordinates (X, Y and Z) that correspond to each collected laser pulse. The point clouds are used to produce multiple products such as digital elevation models, surface canopy-models and contours (NOAA, 2013), which can then be used in an endless number of hydrological, urban, forest and geomorphological applications (Charlton et al., 2003).

\subsubsection{Ground Data}

Ground data collection is ideally conducted concurrently to the remotely sensed data acquisition (McCoy, 2005) and involves the observation, evaluation and measurement, as well as geographic position, of the phenomena under question (Lillesand et al., 2004; Reif et al., 2012). The data collected from the ground is used to assist with the calibration, interpretation, analysis and validation of the remotely sensed data (Teillet, 1997).

Methods and techniques of ground-data collection are highly application specific and are influenced by project details, such as the location and size of the 
area, scale of the final maps and the required accuracy of the final results (McCoy, 2005; Campbell and Browder., 1995). General observations, such as slope gradient, aspect, date, time, a reference photograph and coordinates, are commonly collected for most applications (McCoy, 2005). Observations, such as vegetation species, percent-ground coverage and sky conditions, are slightly more application specific (e.g., forest investigations using spectral mixture analysis).

Spectroscopic ground measurements are often taken, amongst other measurements, to be used as auxiliary information for remote sensing investigations (Jensen, 2005). Sky condition observations are particularly important when collecting spectroscopic ground measurements because constant irradiance is one of a series of major assumption made concerning the accuracy of the measurements (McCoy, 2005; Schapeman et al., 1997).

Collecting appropriate ground data, be it spectroscopic measurements or chemical and physical measurements and observations, require an in-depth knowledge of the biophysical/biochemical variables being collected and the corresponding spectral response. Consideration must also be given to the potential influences of sampling and scaling procedures that may be required for utilization with the image data (McCoy, 2005). 


\subsection{Hyperspectral Data Pre-Processing}

Inconsistencies as a result of sensor, platform and environmental influences make pre-processing, particularly radiometric, geometric and atmospheric correction, essential steps for the extraction of information from hyperspectral data (Chakravortty and Chakrabarti, 2011; Jensen, 2005).

\subsubsection{Sensor Radiometric Calibration}

Sensor radiometric calibration deals with the conversion of incident Digital Numbers (DN) values to radiance, the calibration of the image detector responses and the spectral calibration (Rogass et al., 2011). Sensor radiometric calibration is an ongoing process, involving ground methods prior to launch, onboard methods post-launch (e.g., reference lamps, solar illumination) and vicarious approaches using earth calibration sites and sensor-cross calibration (Teillet et al., 2001; Kastner and Slater, 1983). Uncorrected for, sensor radiometric calibration produces its greatest limitation for quantitative remote sensing applications (Teillet and Coburn, 2010; Teillet, 1986).

\subsubsection{Destriping}

Noise introduced into a remote sensing system interferes with the radiance recorded by the system. With too much noise in the system, the radiance recorded by the system may no longer be representative of the radiance leaving the observed surface. Common forms of noise introduced by the sensor system itself include, for example, shot noise, line start and stop problems, 
full/partial line or column drop outs and line or column striping. Noise is often introduced as a result of the individual detectors not functioning properly or improper calibration (Teillet, 1986).

Striping artifacts are a form of noise and may still remain prior to the radiometric correction due mainly to uncertainties and variations in the calibration of the detector elements (Tsai and Chen, 2008). This is particularly common with pushbroom sensor technology because each detector element is calibrated separately. Even small variations in the calibration of a detector array can result in striping (Rogass et al., 2011).

The incorrect or missing data in the striping can be accounted for by either adjusting for the miscalibration or by using techniques to generate new data (Rogass et al., 2011). Some examples for destriping include histogram matching (Wegener, 1990), inverse regression (Chander et al., 2002), interpolation (Tsai and Chen, 2008), moment matching (Sun et al., 2008) and Fast Fourier Transformation (FFT; Liu and Morgan, 2006). Ideally, the procedure chosen for destriping would perform a recalibration of the data rather than generate new data to maintain their integrity (Rogass et al., 2011).

The goal of destriping is to correct for striping artifacts in the data without altering non-affected pixels (Tasi and Chen, 2008). If not adjusted for, striping will reduce the quality and interpretability of the data (Tsai and Chen, 2008), and 
have a negative effect on classification (Datt et al., 2003; Landgrebe and Malaret, 1986).

\subsubsection{Atmospheric correction}

Atmospheric corrections are used to compensate for the scattering and gaseous effects of the atmosphere in remotely sensed data, such as water absorption and Rayleigh scattering. It should be noted that certain applications, such as particular types of classification and change detection, do not always require atmospheric correction, however it is best practice to atmospherically correct the data (Song et al., 2001; Cracknell and Hayes, 1993). However, atmospheric compensation is necessary when applications require the extraction of physical parameters (Haboudane et al., 2002; Thiemann and Kaufmann, 2002) or when the data are used in variable temporal or spatial studies (Song et al., 2001). Depending on the application purposes, compensation for atmospheric effects can be done in either an absolute or relative manor.

\subsubsection{Relative Atmospheric Normalization}

Relative atmospheric compensation methods are used to normalize the atmospheric effects either among different bands within an image or between bands of different images. Relative correction methods are beneficial when there is not extensive knowledge of the atmospheric or scene characteristics (Hajj et al., 2008). Examples of relative atmospheric normalization techniques include linear regression (Schott et al., 1988) and dark object subtraction (Chavez, 1988). 
Although relative atmospheric normalization methods are beneficial in applications such as time series (Hajj et al., 2008), they are not suitable for applications requiring physical reflectance units.

\subsubsection{Absolute Atmospheric Correction}

The general goal of absolute atmospheric correction is to convert DN values into scaled surface spectral reflectance by removing the unwanted effects of the atmosphere (Griffin and Burke, 2003; Du et al., 2002). Reflectance is the fraction of incoming radiation that is reflected from Earth's surface. Physical reflectance units are necessary to retrieve quantitative values and extract biophysical and biochemical parameters from remotely sensed data (Haboudane et al., 2002; Thiemann and Kaufmann, 2002; Teillet, 1986). Empirical Line Calibration (ELC) is an example of absolute atmospheric correction in which information about the atmospheric parameters are neither required nor derived in the correction process (Griffin and Burke, 2003; Smith and Milton, 1999; Roberts et al. 1986).

However, physics-based methods requiring a priori knowledge of atmospheric and surface characteristics are another approach for full atmospheric correction (Griffin and Bruke, 2003). This is typically achieved using Radiative Transfer (RT) codes that model the propagation of electromagnetic radiation through the atmosphere. Examples of such RT codes include the MODerate resolution atmospheric TRANsmission (MODTRAN; Guanter et al., 
2009; Alder-Golden et al., 1999; Berk et al., 1998) or Second Simulation of the Satellite Signal in the Solar Spectrum (6S; Vermote et al., 1997).

\subsubsection{ATCOR}

Atmospheric and Topographic Correction (ATCOR) for airborne imagery is an atmospheric correction software based on MODTRAN. It performs the combined atmospheric and topographic correction of remote sensing imagery taking into account the atmosphere, solar illumination, sensor viewing geometry and terrain information (Richter, 2004; Richter and Schläpfer, 2002). Surface reflectance is derived using a series of look up tables of the atmospheric correction functions calculated using MODTRAN, such as path radiance, atmospheric transmittance, direct and diffuse solar flux, and DEM derived terrain shape (Richter and Schläpfer, 2002). Examples of other approaches that correct for both atmospheric and topographic effects are offered in Sandmeier and Itten (1997) and Staenz and Williams (1997).

\subsubsection{Geometric Correction}

The objective of geometrically correcting a remotely sensed image is to link pixels in the image to coordinates on the ground (Lee and Bethel, 2001). Due to advancements in off-nadir viewing and finer spatial resolution (Chong and Pearson, 1998), as well as temporal and multi-source data integration and quantitative information extraction (Rocchini and Di Rita, 2005) geometric 
correction is becoming a greater focus in the pre-processing of remotely sensed data (Toutin, 2004).

The sources of geometric distortions can originate both internally and externally of the sensing system. Distortions relating to the sensing system as a whole include variations in platform movement and miscalibrations of the Inertial Measurement Unit (IMU) and Global Positioning System (GPS) devices. Characteristics of the Earth, such as the rotation, curvature and topography, are examples of external sources (Toutin, 2004). The sources and magnitudes of distortion also vary greatly based on factors such as the type of sensor (Visible/Infra-Red (VIR), Synthetic Aperture RADAR (SAR), high or low resolution), the Field of View (FOV; Toutin, 2004; NRC, 2008; Schläpfer et al., 1998). The type of platform used for data collection also affects the magnitude of the distortions. The issue of distortions introduced into the image data as a result of the platform is a much more complex problem with airborne data than it is with satellite platforms.

The process of georectifying an image includes; 1 ) the compensation for the image distortions (geometry), and 2) the resampling of the data, which assigns spectral values to the pixels. Two techniques can be used for the geometric correction: 1) deriving mathematical relationships between the image pixel and the corresponding coordinate on the ground, or 2) by modeling the nature and magnitude of the sources of distortion (Richards, 2013). 
The former technique is often chosen when the parameters of the image acquisition required for a physical model are not available (Toutin, 2004).

Accordingly, this model does not reflect or correct directly for the source of the distortions and the models have no actual physical meaning (Toutin, 2004). These techniques often use polynomial functions to warp the imagery in a way that will account for the distortions present.

Coordinates used in the polynomial functions are gathered using ground control points, which relate a feature in the image to the corresponding feature on a map or image of known coordinates (Richards, 2013). First, second and third order polynomials are commonly used in geometric corrections.

First order polynomials are able to account for simple rotation, scaling, translation and obliquity of the image, whereas a second order polynomial adds to those movements by also allowing for corrections of torsion and convexity (Toutin, 2004). Third order polynomials, although providing lower error estimates often introduce errors in the form of image contortions in areas of the image that have been poorly defined by the polynomial (Toutin, 2004). It has been demonstrated that polynomial functions are quite powerful with flat terrain imagery. However, they have been found to be inappropriate for cartographic applications (Rocchini and Di Rita, 2005) due to their poor performance in rugged terrain imagery (Cheng et al., 2000) as well as with airborne imagery. In the case of airborne imagery, triangulation is often suggested (Exelis, 2014c). In 
rugged terrain applications, it is suggested a more powerful geometric correction method be applied such as orthorectification (Rocchini and Di Rita, 2005).

Orthorectification is a reprojection of an image excluding the influence of topography. It is not only more powerful in rugged terrain situations than polynomial functions, but it is also more appropriate when dealing with airborne imagery (Schläpfer, 1998). The distortions in airborne imagery as a result of the complex flight path and attitude movements cannot be approximated accurately by polynomial transformations (Schläpfer, 1998).

The auxiliary data used in the modelling of the geographic distortions is commonly gathered from the GPS and IMU systems onboard the aircraft. These systems provide information on the platform (position, velocity and attitude), sensor (viewing angles and panoramic effect), earth (ellipsoid and relief for 3D) and cartographic projections (Toutin, 2004). Being able to account for, not only the platform and sensor distortions, but also the relief and topographic distortions is important, because elevation and slope are terrain characteristics that can seriously impede the final accuracy of the geometric correction (Paine, 1981). There are various different types of 3D physical models available, many of which are listed in Toutin 2004.

Parametric Geocoding and Orthorectification (PARGE) for airborne optical scanner data is an example of a program that offers a 3D orthorectification which fully reconstruct the geometry of the scanning process 
(Schläpfer et al., 1998). Similarly to most 3D orthorectification software, PARGE is based on the collinearity condition and equations (Schläpfer et al., 1998), which defines the geometry connecting the center of a sensor, an image point in two dimensions and an object on the ground in three dimensions (Wong, 1980; Konecy, 1972).

Once the image geometry has been corrected, the image then needs to be resampled to a regular grid. This is the second process in the geometric correction of image data. The common resampling methods used in remote sensing are Nearest Neighbour (NN), Bilinear Interpolation (BI) and Cubic Convolution (CC). NN resamples the data by assigning the newly transformed pixel the value of the nearest pixel from the input layer. With NN none of the cell values from the original input layer are changed and for this reason is commonly used with categorical or integer data. BI resamples the data by using a weighted average of the four nearest cells. With BI the values from the original input layer are not maintained. CC uses the surrounding 16 pixels to determine the pixel values in the output grid. The resulting image when resampling using CC often has a much smoother appearance than when resampling using NN or BI. Each resampling method has its benefits and drawbacks. NN is often preferred when working with spectrometry data, because the original spectra are maintained from the input layer which in turn maintains the spectral integrity of the data (Schläpfer et al., 2000). However, NN can also be associated with a loss of spatial 
quality and smoothing due to resampling artifacts (Schläpfer et al., 2000). This results from the reality that, once the data has been corrected for distortions, an original image pixel may be the nearest neighbour for more than one of the corrected image pixels. Consequently, this results in the smoothing of features in the image. CC, on the other hand, tends to alter the brightness values from the original input and for this reason is not recommended if classification is to follow (Richards, 2013).

Assessing the accuracy of the geometrically corrected data against a known source is necessary for understanding the outcomes of future image processing steps. Geometric accuracy is often evaluated using the Root Mean Square Error (RMSE; McGwire, 1996). The RMSE is a measure of average error, calculated using the observed and forecasted (or actual) value of a number of validation points. Calculation of RMSE for an image uses the calculated distance between Ground Control Points (GCP) from a map, representing the actual location of the point, and the corresponding GCPs on the transformed image.

Unfortunately RMSE is not the best indicator of geometric accuracy because it can be influenced by many external factors, such as poor location and distribution of GCP, making it a poor indication of true registration accuracy (McGwire, 1996). For this reason, it is suggested to gather as many GCPs as possible when calculating the RMSE of an image in hopes of accounting for possible errors in location and unevenness of distribution. As well, substituting 
GPS points for the map GCPs has been suggested for improving the accuracy of the RMSE calculation (Cook and Pinder, 1996), which by improving the accuracy of the error calculation can give further insight into the limitations of the data and data derived products (Townshed at al., 1992).

\subsubsection{Bidirectional Reflectance Distribution Function (BRDF)}

Reflectance anisotropy is defined as the directionally dependent intensity of reflected light. This can be described more simply as changes in the reflectance of an object (e.g., a particular land-cover) when viewed from different directions. The Bidirectional Reflectance Distribution Function (BRDF) is used to describe reflectance anisotropy. It is defined by Niodemus (1977) as a distribution function, relating the irradiance incident from one given direction to its contribution to the reflected radiance in another direction. The BRDF can be derived from goniometric measurements which, in remote sensing, are used in combination with a sensor to measure the reflectance of an object from all possible angles (Coburn and Peddle, 2006; Sandmeier et al., 1998).

BRDF effects depend on illumination and viewing geometry as well as wavelength (Coburn and Peddle, 2006; Beisl and Woodhouse, 2004). These effects, as well as radiometric distortions (as well as other effects, such as object displacement), are becoming a much larger issue with the introduction of wider FOV sensor systems (Feingersh et al., 2007; Schiefer et al., 2006). An example of a 
BRDF effect that is found exclusively in pushbroom sensors is a brightness gradient in the across-track direction (Schiefer et al., 2006).

The radiometric bias introduced into remotely sensed imagery by BRDF effects, such as the aforementioned across-track brightness gradient, interferes greatly with processing and information extraction from remotely sensed imagery. Without correction, the across-track brightness gradient prevents accurate comparison within and between images, effects spectral ratios such as vegetation indices, complicates image mosaicking, hinders spectral library and field spectra integration and can confuse image classification (Schiefer et al., 2006; Beisl and Woodhouse, 2004). Furthermore, BRDF introduces uncertainty into quantitative analysis of hyperspectral imagery (Ben-Dor et al., 2010; Feingersh et al., 2007; McDonald et al., 2002).

Correction for BRDF is complicated as a separate correction for each landcover type is required for producing accurate results (Richter and Schläpfer, 2012; Beisl, 2001; Kennedy et al., 1997). It is further complicated by topographic influences on illumination intensities and local variations of the angular positions of the Sun and the angular variation within the FOV (Feingersh et al., 2010; Feingersh et al., 2007). Accordingly, Feingersh et al., (2007) proposed that two pieces of information are needed to properly correct for BRDF; 1. A detailed solar-ground-sensor geometry model and 2. a calculated anisotropy dataset for the land-cover of interest. 


\subsection{Sensor Simulation}

Sensor simulations are used in the scope of both existing and future sensor systems (Parente et al., 2010; Sandberg et al., 2005; Verhof and Bach, 2003; van

der Meer et al., 1999). Existing sensors are often simulated used to determine the detection capabilities and mapping accuracies under varying scene characteristics. Such was the purpose of the post-launch simulations for the Compact Reconnaissance Imaging Spectrometer for Mars (CRISM) sensor (Parente et al., 2010).

SPOT is a sensor system that was thoroughly simulated pre-launch for the purpose of evaluating the land-use/ land-cover application capabilities (Betts et al., 1986; Buchan and Hubbard, 1986; Essery and Wilcock, 1986). Sensor simulations used in the scope of future satellite systems are largely used for evaluating detection and mapping capabilities (Peisker et al., 2010; Segl et al., 2010; Aktaruzzaman, 2008; Verhof and Bach, 2003; van der Meer et al., 1999; Kerekes and Landgrebe, 1988; Betts et al., 1986; Buchan and Hubbard, 1986) and, for the purpose of trade-off studies for defining system parameters (Cota et al., 2010; Kaufmann et al., 2006; Sandberg et al., 2005; Broner et al., 2001).

Additionally, as with the SPOT simulation campaign, pre-launch simulated data are also used to find and develop a market for the data products (Binger and Ory, 1984). 


\subsubsection{Sensor Simulations - Future Sensors}

Sensor simulations for the investigation of future sensor systems are focused on producing sensor-like output data by modelling the characteristics, both known and estimated, of the sensor being investigated (Segl et al., 2012). Simulation end products are often in the form of Top-of-Atmosphere (TOA) DN values. The process of simulating artificial raw sensor data from existing reflectance data is commonly considered an "end-to-end" simulation. Examples of end-to-end simulations include the Parameterized Image Chain Analysis and

Simulation Software (PICASSO; Cota et al., 2008), Software ENvironment for the Simulation of Optical Remote sensing systems (SENSOR; Broner et al., 2001), Surface Processes and Ecosystem Change Through Response Analysis (SPECTRA; Verhoef and Bach, 2003), and EeteS (Segl et al., 2012). Simulation software package will be described further in sections 2.4.2 and in the methods chapter.

\subsubsection{EeteS Sensor Simulation}

EeteS is a sensor simulation program which was developed at the Helmholtz Centre Potsdam, German Research Centre for Geoscience (GFZ), Potsdam, in the framework of the EnMAP satellite mission (Segl et al., 2012). Similarly to the aforementioned simulation software packages, they use existing spectrally and spatially oversampled data to produce simulated raw data. However, in the scope of the EeteS simulation software, an "end-to-end" 
simulation includes both the simulation of TOA DN values (forward simulation) and the conversion from DN values to orthorectified reflectance values (backwards simulation) (Segl et al., 2012). Included in the EeteS simulation process is also the incorporation of sensor and calibration errors (Guanter et al., 2009). This inversion process is necessary for realistic analyses of the simulated data, especially with a highly detailed sensor model such as used in EeteS (Segl et al., 2012; Segl et al., 2010; Guanter et al., 2009).

EeteS simulated data products have been used for optimization of instrument design, in sensitivity analysis on the effects of different environmental and instrument parameters, and as a test bench for the development of algorithms for future information products (Segl et al., 2012; Segl et al., 2010; Peisker et al., 2010; Guanter et al., 2009).

\subsection{Hyperspectral Image Processing}

The end goal of image processing is the extraction of information from the hyperspectral image cube. Image processing is carried out using algorithms which, for example, can extract biophysical/biochemical information or thematic maps from the data (Jensen, 2005). The information can then be introduced directly to application purposes or can be used for modelling purposes.

Although some techniques used for multispectral image processing can be applied to hyperspectral imagery, they are usually not capable of exploiting the hyperspectral data's full potential (Plaza et al., 2009). Algorithms designed for 
hyperspectral data take advantage of the high-spectral dimensionality and are able to provide quantitative information such as fractional cover (sub-pixel classification) (Peddle et al., 1999).

The need to quantify surface cover at a sub-pixel level results primarily from larger spatial resolution images and highly variable surface covers. Unfortunately, even though compositional information can be derived for an individual pixel using these algorithms, the spatial component defining the distribution of these components with in the pixel is lost (Plaza et al., 2009).

\subsubsection{Linear Spectral Unmixing}

Linear spectral unmixing is based on the assumption that a variety of surface covers reside within a single pixel (Tseng, 2000). These pixels are referred to as mixed pixels. The spectral signature collected from a mixed pixel is commonly assumed to be a linear combination of the spectral response of each surface component within the pixel (Keshava and Mustard, 2002). Variations from the linear mixture model include the non-linear mixture model, which refers to the interaction of light with multiple target materials (Ray and Murry, 1996).

What linear spectral unmixing attempts to do is estimate the abundance (or fraction) of each surface cover within a pixel by decomposing its spectral signature using a known set of scene endmembers. Endmembers referring to a 
set of spectrally pure signatures representing each surface cover within an image (Du et al., 2008; Plaza et al., 2004).

$\mathrm{S}_{\mathrm{ij}}=\mathrm{X}_{1} * \mathrm{~S}_{1}+\mathrm{X}_{2} * \mathrm{~S}_{2}+\cdots+\mathrm{w}$

where $S_{i j}$ is the surface reflectance of the pixel at $i j, i$ is the image pixel number, $j$ is the image line number, $X_{n}$ is the abundance (fraction) of spectrum $S_{n}, S_{i}$ is the nth endmember spectrum, and $\mathrm{w}$ is the error term.

Outputs from spectral unmixing are a series of abundance images (equal to the number of endmembers used to unmix the scene) and a Root Mean Square (RMS) error image. The abundance images show the spatial distribution of each endmember based on its abundance within each pixel. The RMS error image identifies areas and degrees of error associated with the unmixing of each pixel based on how well the pixel was modelled by the set of endmembers.

When applying linear spectral unmixing, three main levels of constraint can be placed on the resulting fraction images: unconstrained, constrained and weakly constrained. Unconstrained unmixing does not apply any constraints to the fraction values, which is beneficial in as it gives the most accurate representation of the applicability of the model. However, the abundances can range between values below 0 and above 1 , and the sum of the fractions for a pixel are not required to sum to unity (Exelis, 2014b). Constrained unmixing applies constraints both on to the range of fraction values for each surface cover 
as well as constrains the pixel sum to unity. Weakly constrained unmixing is a hybrid of the previous two methods which constrains the fraction values between 0 and 1 and allows the sum of the pixel to fluctuate $<1$. This in turn eliminates the requirement to have all spectral endmembers for the unmixing procedure (Staenz et al., 2001).

Since traditional pixel-by-pixel accuracy assessment is not suitable for unmixing results (Shang et al., 2008), the validity of the results is determined using a set of criteria specific to the abundance images and the RMS error image. The incompatibility between pixel-by-pixel accuracy assessment and unmixing results is because traditional accuracy assessments can only assign one class for each pixel, whereas unmixing results identify within pixel fractional cover of multiple classes. These criteria state that the fraction values range between 0 and 1 (for constrained and weakly constrained), the average RMS error is low and there is a low spatial correlation in the RMS error image (van der Meer, 1995).

Settle and Drake (1993) addressed four assumptions that are made when using linear spectral unmixing. These four assumptions are: 1) There is no significant occurrence of multiple scattering; 2) there is sufficient spectral contrast between surface components; 3) each pixel sums to unity; and 4) all image endmembers are known. Each of these assumptions is key to successfully applying linear spectral unmixing to an image. Failure to meet these assumptions 
could result in incorrect modelling of the scene, rendering the unmixing results unreliable.

\subsubsection{Endmember Selection}

An endmember is the reflectance spectrum related to a pure surface cover within an image scene (Tompkins et al., 1997; Bateson and Curtiss, 1996). Representative endmembers are collected for all pure target materials in an image. The complete set of endmembers is then used as the fundamental components in the mixture modelling of image pixels.

Endmember spectra can be collected from the image or from external sources such as in the laboratory or in the field. A major difference between endmembers acquired externally and those collected from the scene is that the external endmembers may not have been collected under similar conditions as the image data (Plaza et al., 2004). In the same way that image derived endmembers have the benefit of being collected under the same conditions, they also have the benefit of being collected at the same spatial scale as the image making them easier to associate with the image (Plaza et al., 2004). However, the ability to collect pure endmembers from within an image is complicated if the spatial resolution is too large, making it less likely that only a single surface cover is contained within the pixel boundaries.

Difficulty often occurs when there are unknowns concerning how many endmembers are within a scene and what surface materials define those 
endmembers. One method identified for estimating the number of endmembers within a scene is carried out using information derived from a Principal Component Analysis (PCA; Smith et al., 1985). It has been proposed that the number of endmembers within an image scene is equal to $N+1$, with $N$ equalling the number of useful eigenvectors collected from the PCA.

Identifying the actual endmembers from within the image scene can be done using a variety of methods. Included in these methods are visualizing endmembers in N-Dimensional (N-D) space (Matinez et al., 2006), the Pixel Purity Index (PPI; Boardman et al., 1995) and the Sequential Maximum Angle Convex Cone (SMACC; Gruninger et al., 2004). Many of the methods used to extract endmember spectra from within a scene are based on identifying the most 'extreme' pixels (Veganzones and Grana, 2008), equating extreme to pure. The disadvantage to this assumption is that noise can often severely affect endmembers identifications (Boardman et al., 1995).

The identification of accurate endmembers is essential to the accurate unmixing of the image data. However, this is complicated due to endmember signature variability (Schowengerdt, 2006). As a result, the identification and extraction of endmember has been acknowledged as being the most difficult procedure in the unmixing process (Tompkins et al., 1997). 


\subsubsection{Multiple Endmember Spectral Mixture Analysis (MESMA)}

MESMA is built upon the foundations of basic linear spectral unmixing. However, it is adapted to compensate for endmember spectral variability (Roberts et al., 2007). Spectral mixture analysis uses an invariable set of endmembers (Myint and Orkin, 2009). MESMA, on the other hand, allows for the number and type of endmembers to vary on a per-pixel basis (Roberts et al., 1998). Since MESMA allows for within endmember spectral variability, it is particularly beneficial in environments where endmember composition, such as with soils or urban environments, changes rapidly over space (Franke et al., 2009; Palacios-Orueta et al., 1999).

Up to four endmembers (one being shade) can be selected for unmixing in MESMA. Within each of the endmembers, a series of spectra can be contained. This is how MESMA accounts for within class spectral variability. It works by applying a series of candidate unmixing models to the image on an individual pixel basis. These candidate-unmixing models are derived from all the possible combination of two, three and four endmembers chosen for the scene. The model that is chosen to unmix each pixel is selected based on minimum pixel fraction error (RMSE) (Roberts et al., 2007).

A series of constraints can be placed on the fraction outputs including a fraction constraint model, which determines the minimum and maximum allowable fraction values and an RMSE constraint, which removes any model 
that exceeds the maximum RMSE threshold value. Outputs from MESMA are similar to basic Spectral Mixture Analysis (SMA). However, it also includes a classified image, which shows the spatial distribution of each model selected for each pixel.

Care must be taken when selecting endmember spectra for use in MESMA, because accommodating spectral variability can sometimes result in the selection of many endmember spectra, which will drastically increase computational times. When selecting endmember spectra the goal is to choose spectra that are the most representative of the particular class while remaining highly separable from the other classes (Roberts et al., 2007).

Methods have been developed that essentially model potential composition of sub-scene elements of the input scene. These methods work directly with MESMA to help with the selection of endmembers. These are the Count-based Endmember Selection (CoB; Roberts et al., 2003), Endmember Average RMSE (EAR; Dennison and Roberts, 2003) and the Minimum Average Spectral Angle (MASA; Dennison et al., 2004).

\subsection{Remote Sensing of Soils}

Spatial and temporal variations in soil properties are the basis for many Earth and atmosphere process models (King et al., 2005; Mosier, 1998). Traditional approaches for collecting spatial data about soil properties and conditions is based largely on direct sampling and subsequent laboratory 
processing (Helming et al., 1998; Merrill, 1998; Podmore and Huggins, 1981).

However, these traditional methods are rarely able to account for all the different spatial and temporal scales at which soil properties vary and soil processes occur (Hopmans et al., 2002).

Remote sensing techniques benefit from the large spatial coverage and, in the case of satellite sensors, temporal revisit time, which can provide the required information for many Earth-atmosphere models and soil mapping studies (Asner and Heidebrecht, 2003). Continued advancements in quantitative analysis and multi-sensor approaches expand the applicability of remote sensing to soil investigations (Ben-Dor, 2002; Moran et al., 2002). However, limitations do exist in the application of passive remote sensing to soil investigations. Examples of these include the ability to only detect the surface soil layer (Grunwald, 2010; Ben-Dor et al., 2002; Ben-Dor et al., 1999), which is further complicated by vegetation cover (Okin et al., 2001). Complications also exist as a result of atmospheric distortions (Ben-dor et al., 2009; Bodechtel, 2001) and BRDF effects within the image (Torrent and Barron, 1993).

\subsubsection{Soil Imaging Spectroscopy}

Hunt and Salisbury (1971) pioneered the interest in spectroscopy for the mineral composition in soils in the 1970's and the interest was extended by Stoner and Baumgardner (1980) in the 1980's with the development of the American Soil Spectral Library (Chabrillat et al., 2013). Spectroscopy has become 
so popular for soil studies, because it is a quick, non-destructive method to derive predictions about the soil's physical, chemical and biological properties (Viscarra Rossel et al., 2006; Reeves et al., 1999; Janik et al., 1998; Ben-Dor and Banin, 1995).

Conventional methods of soil analysis involved the drying and crushing of the soil sample, as well as the occasional application of chemicals, which alters the integrity of the soil (Viscarra Rossel et al., 2006). Soil spectroscopy provides a less damaging and less expensive means of estimating soil properties than conventional methods (Chabrillat et al., 2013).

The soil's spectral signature originates from the combinations of minerals, organic matter and water molecules comprising the soil (Irons et al., 1989). It is the scattering and absorption properties as well as the arrangement of these soil properties that the spectral reflectance of a soil is derived from (Weidong et al., 2002). Also contributing to the spectral reflectance are soil characteristics, such as the particle size and surface roughness (Chabrillat et al., 2013; Wang et al., 2012). The Visible Near Infra-Red (VNIR) and the Short-Wave Infra-Red (SWIR) are both sensitive to particular properties within a soil's composition. The VNIR contains information on soil colour, iron content and composition, soil water and organic matter. The SWIR provide a larger portion of the information on the soils mineral content, including phyllosilicates, sulphates, amphiboles and carbonates, as well as soil water hydroxides and organic matter (Chabrillat et al., 2013; 
Shepherd and Walsh, 2002). Identifying and quantifying soil components such as clay, soil organic matter, carbonates and salt is important, because they can give an indication about the fertility and erodibility of the soil (Melendez-Pastor et al., 2008; Horn and Baumgartl, 2002; Hillel, 1980).

\subsubsection{Digital Soil Mapping}

Traditional soil mapping is based on thorough field survey that includes detailed descriptions of the soil profiles (chemical, physical, development, origin and formation) and is often complimented by laboratory analysis (Soil Survey Staff, 1999). Current needs for soil data require that they are accurate and inexpensive (Stenberg et al., 2010) which, due to its laborious and subjective nature, cannot be provided by traditional surveys (Beckett and Burrough, 1971). Digital soil mapping, on the other hand, can provide not only a quantitative characterization of the observed soils, but also a measure of accuracy to accompany the results (Scull et al., 2003).

Hyperspectral data has been found to be much more suitable than broadband imagery as acquired with SPOT HVR and Landsat Thematic Mapper (TM) for mapping soil properties due to the much narrower spectral bandwidths, which are capable of detecting key diagnostic absorption feature present in soil spectra (De Jong, 1994). The application of hyperspectral data to digital soil mapping often employs methods that are found in a laboratory setting, and applied within a spatial domain (Chabrillat et al., 2013; Ben-Dor et al., 2009). 
Generating a digital soil map from remotely sensed data can be done using many methods. Modelling is one method of digital soil mapping, which employs mathematical functions to make predictions about the soil condition/characteristics based on known surface properties (Bouma and van Lanen, 1987; Lamp and Kneib, 1981). Another method uses a geostatistical approach, which interpolates soil attributes using field samples, analysed data, or a combination of both (Matheron, 1962). Finally, a digital soil map can be generated by classifying the remotely sensed image based on similar soil attributes/characteristics (Behrens and Scholten, 2006; Moore et al., 1993). A limitation of remote sensing in regards to digital soil mapping is that only the surface soil condition/characteristics can be identified. As a result, information about the entire soil profile, which is required to provide a full and detailed soil map is lost (Grunwald, 2010; Ben-Dor et al., 2002; Ben-Dor et al., 1999).

In addition to the inherently complex nature of soil reflectance, complications also arise as a result of the intrinsic principles of remotely sensed imagery. Interactions of the electromagnetic radiation with the atmosphere and noise within the data can cause distortion in the spectra which, if not adequately compensated for, can mask or be mistaken for important mineral absorption features (Ben-dor et al., 2009; Bodechtel, 2001). As well, anisotropy (BRDF) of the surface captured in a remotely sensed image can influence the assessment of deterministic soil properties such as soil colour (Torrent and Barron, 1993; Wang 
et al., 2012). However, with the introduction of high-SNR sensors, particularly in the SWIR, soil spectroscopy is becoming increasingly applicable (Chabrillat et al., 2013).

\subsubsection{Soil Erosion Mapping in Semi-Arid Regions}

Semi-arid zones are prone to sever erosion as a result of the climatic conditions and geomorphological processes in the regions (Shrestha et al., 2005). The erosional features found in semi-arid zones are often quite similar to desert features (Rapp, 1986).

Soil erosion mapping using remote sensing employs the same methods mentioned previously; however, the soil properties under investigation relate to the soil erosion features characteristic of the region. It is these desert-like features that are often used in remote sensing to detect and map soil erosion in semi-arid Mediterranean regions. These desert-like features include desert pavement and surface/biogenic crusts (Escribano et al., 2010; Shrestha et al., 2005; Margate and Shrestha, 2001), salinization which can be identified by a series of spectral features (e.g., 800 - $810 \mathrm{~nm}$ ) (Shrestha et al., 2005; Raina et al., 1993), gypsum content which is identified by absorption at $1800 \mathrm{~nm}$ and $2300 \mathrm{~nm}$, and finally, carbonate content which can be identified by absorptions at $2350 \mathrm{~nm}$ (Shrestha et al., 2005). Carbonate content is a particularly prominent identifier of soil erosion in semi-Mediterranean regions, because it comprises a large component of the regional parent rock and, therefore, its presence at the surface indicates either 
weakly developed soils or the removal of developed soils (Hill et al., 1994; Barth, 1982).

Although these indicators of soil erosion can be effectively identified using remote sensing techniques, the heterogeneity of semi-arid Mediterranean environments makes the mapping of these features complicated (Schlesinger and Pilmanis, 1998; Puech, 1994). One of the main complications that arises is the creation of mixed pixels that result due to the complex mixtures of soils, rock and vegetation as well as due to the high-spatial variability of the soils themselves (Escribano et al., 2010; Stenberg et al., 2010; Puech, 1994; Tueller, 1987).

Spectral unmixing is spectral decomposition methods often used to deal with the mixed pixel problem in semi-arid soil investigations (Schmid et al., 2012; Tromp and Epema, 1998). When employing spectral decomposition methods, it is increasingly important to have detailed knowledge of the natural variability (compositionally, spatially, temporally, etc.) of all surface components as well as an understanding of the spectral variability associated with each (Escribano et al., 2010). This is because the accuracy of such decomposition techniques relies largely on the ability to identify the spectral variability within the scene (van der Meer, 2002).

\subsubsection{Considerations in Agricultural Regions}

Vineyards, olive groves and rain-fed cereal crops dominate the main agricultural land-uses in Spain (Garcia-Ruiz, 2010). They are of particular interest 
in soil erosion studies because they are all highly susceptible to erosion either due to extensive exposed soil surface during the growing period or because they are left exposed for extended periods of time due to years of fallow (Garcia-Ruiz, 2010; De Graaf and Eppink, 1999; Garcia-Ruiz et al., 1995). However, there is difficulty in assessing these areas for soil erosion using remote sensing because the soil signal is often masked in mixed pixels due to the overlying vegetation (Daughtry, 2001; Roberts et al., 1993). Consequently, segregation of the vegetation dominated pixels from the soil-dominated ones is considered the first step to soil mapping in such situations (Hill and Schutt, 2000; Palacios-Orueta et al., 1999; Palacios-Orueta and Ustin, 1998).

Spectrally, green vegetation and soils are distinct. Green vegetation has a low reflectance in the Visible (VIS) due to chlorophyll absorption, a high reflectance in the Near Infra-Red (NIR) which is related to leaf structure, and lower reflectance in the SWIR which is mainly due to water absorption (Escribano et al., 2010). However, discriminating between plant litter or senesced vegetation from soils is more complicated due to the spectral similarity in the VNIR (400 nm - $1100 \mathrm{~nm}$ ) (Nagler et al., 2000). Nevertheless, discrimination of senesced vegetation from soils is possible using the cellulose and lignin absorption features located at $2100 \mathrm{~nm}$ and $2300 \mathrm{~nm}$ (Figure 2.1) (Daughtry et al., 2004; Nagler et al., 2003; Elvidge, 1990; Curran, 1989). 


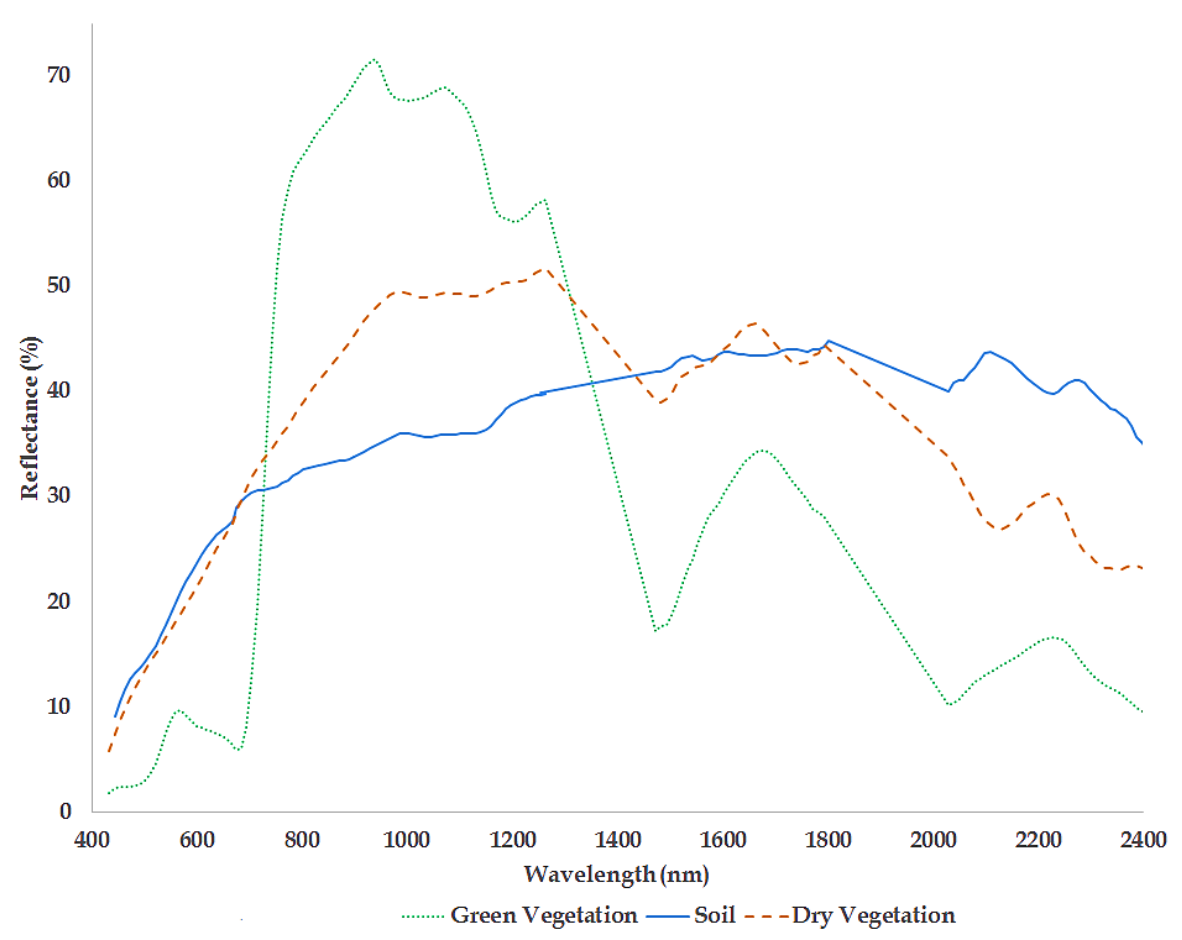

Figure 2.1: Comparison of green vegetation, dry vegetation and soil spectra.

When discriminating vegetation cover from soils, it has been noted that any pixels with a vegetation proportion exceeding $30 \%$ should be excluded from further soil analysis (Bartholomeus et al., 2007; Chabrillat et al., 2002; Tueller, 1987). Discrimination of vegetation cover from soils can be done using many methods such as Vegetation Indices (VI) (e.g., Normalized Difference Vegetation Index (NDVI; Hurcom and Harrison, 1998) and Cellulose Absorption Index (CAI; Nagler et al., 2003)) or spectral mixture analysis (De Asis and Omasa, 2007; Tromp and Epema, 1998; Roberts et al., 1993). 
Although these are widely used methods for discriminating between vegetation and soils, there are still concerns with the performance of these methods. NDVI values have been found to become unpredictable when the green vegetation cover of a pixel is below 80 \% (Liu and Kafatos, 2005), introducing uncertainty in to the results. The CAI, and in particular the cellulose feature at $2100 \mathrm{~nm}$, becomes unreliable in instances where the plant litter has a high water content or when the litter decomposes (Serbin et al., 2009; Daughtry, 2001).

The results of spectral mixture analysis are largely dependent on the understanding and accurate representation of the scene variability (van der Meer, 2002). Another consideration regarding the use of this approach for discriminating vegetation cover deals with the general assumption of linear mixing modeling the pixels, when in fact it is largely a non-linear mixing between soils and vegetation (especially desert-like vegetation) (Ray and Murray, 1996; Roberts et al., 1993; Smith et al., 1990; Huete et al., 1985).

\subsection{Terrain Analysis for Soil Applications}

Terrain information, typically in the form of a DEM, can provide substantial benefits when combined with optical remote sensing data (Florinsky, 1998; Dobos et al., 2000; Haboudane et al., 2002). Not only can a DEM be used to correct for distortions caused by topographic variations, it can also provide 
additional data, such as slope, curvature and aspect, for modelling and analysis purposes (Franklin, 1987; Lee et al., 1988; Yuan et al., 1994).

The additional data provided by a DEM have been found to greatly increase classification accuracy (Franklin, 1987). With soil applications in particular, topographic variations can be used as indicators of soil properties, soil erosion class and productivity (Chen et al., 2007; Florinsky et al., 2002; Chen et al., 1997; Daniels et al., 1985). Table 2.1 provides a description of certain topographic primary and secondary attributes.

Table 2.1: Description of topographic variables.

\begin{tabular}{|c|l|l|l|}
\hline $\begin{array}{c}\text { Topographic } \\
\text { Variable }\end{array}$ & \multicolumn{1}{|c|}{ Description } & \multicolumn{1}{|c|}{ Details } & \multicolumn{1}{c|}{ Source } \\
\hline Slope & $\begin{array}{l}\text { The rate of change } \\
\text { of elevation }\end{array}$ & $\begin{array}{l}\text { Wilson and } \\
\text { Gallant, 2000 }\end{array}$ \\
\hline Plan Curvature & $\begin{array}{l}\text { The horizontal } \\
\text { plane of a contour } \\
\text { line }\end{array}$ & $\begin{array}{l}\text { In radians. } \\
>0=\text { divergence } \\
<0=\text { convergence }\end{array}$ & $\begin{array}{l}\text { Florinsky et al., } \\
2002\end{array}$ \\
\hline Profile Curvature & $\begin{array}{l}\text { Vertical plane of a } \\
\text { flow line }\end{array}$ & $\begin{array}{l}\text { In radians. } \\
>0=\text { acceleration } \\
<0=\text { deceleration }\end{array}$ & $\begin{array}{l}\text { Florinsky et al., } \\
2002\end{array}$ \\
\hline $\begin{array}{c}\text { Curvature } \\
\text { Upslope }\end{array}$ & $\begin{array}{l}\text { Curvature of the } \\
\text { surface }\end{array}$ & $\begin{array}{l}\text { Can be positive or } \\
\text { negative with zero } \\
\text { representing flat }\end{array}$ & $\begin{array}{l}\text { Wilson and } \\
\text { Gallant, 2000 } \\
\text { Area }\end{array}$ \\
$\begin{array}{l}\text { pixels that flow } \\
\text { into a single pixel } \\
\text { on a DEM }\end{array}$ & & $\begin{array}{l}\text { Wilson and } \\
\text { Gallant, 2000 }\end{array}$ \\
\hline
\end{tabular}

Process-based relationships between terrain and soils are particularly strong in hilly terrain; however, as a landscape ages, the relationship between 
soil variables and the terrain becomes increasingly unpredictable (Wilson and Gallant, 2000). The predictive capacity of the terrain can also be diminished when the spatial resolution of the DEM is low (McKenzie and Ryan, 1999; Gessler et al., 1995). For erosional landscapes, it has been found that this threshold is reached at a 40-m resolution, indicating that erosional studies require a DEM with pixels of $40 \mathrm{~m}$ or less (Gessler, 1996).

\subsection{Conclusion}

Soils serve as an indispensable component of any functioning ecosystem (Lal, 2013; Doran and Parkin, 1994). Accelerated soil erosion is a form of land degradation, which decreases the capacity of the soil to perform its essential atmospheric, hydrospheric and lithospheric functions (Stewart and Lal, 1992). Semi-arid Mediterranean regions are highly susceptible to accelerated soil erosion due to the high rainfall intensity, low average annual precipitation, steep slope, fragile soils and agricultural practices (Garcia-Ruiz, 2010;Thornes and Wainwright, 2003; Kosmas et al., 2000; Garcia-Ruiz, 2010; Poesen and Hooke, 1997).

The assessment, monitoring and management of the vital soil resources is necessary for the continued prosperity of human populations (Morvan, et al., 2008; Yang, et al., 2003; Doran, 2002). Remote sensing provides an efficient and cost-effective method for collecting the information required to successfully accomplish this (Asner and Heidebrecht, 2003). Hyperspectral remote sensing 
data is particularly useful for soil investigations due to its highly detailed spectral information (Deventer van et al., 1997; De Jong, 1994; Irons et al., 1989). Essential to the extraction of information from hyperspectral data is their radiometric, atmospheric and geometric correction (Chakravortty and Chakrabarti, 2011; Jensen, 2005). Algorithms such as linear spectral unmixing take advantage of the high spectral dimensionality inherent of hyperspectral imagery and are able to provide within pixel estimates of surface cover abundance. This is necessary for many soil investigations within semi-arid Mediterranean environments, because they are dominated by highly heterogeneous surface cover (Schlesinger and Pilmanis, 1998; Puech, 1994).

Complications do arise with the remote sensing of soil mainly due to overlying vegetation cover (Okin et al., 2001), BRDF effects (Torrent and Barron, 1993), and atmospheric and sensor artifacts (Ben-dor et al., 2009; Bodechtel, 2001). However, inclusion of auxiliary digital terrain data, has proven to be an asset to any soil investigation due to the high predictive relationship between environmental variables and soil properties which can provide further information about soil state (Wilson and Gallant, 2000; Gessler et al., 1995; Odeh et al., 1993) and, in combination with hyperspectral data, has shown to improve classification accuracy (Franklin, 1987). 


\section{Methods}

Soil management is a key for many of Earth's atmospheric, hydrospheric and lithospheric functions. Assessing and monitoring the current and future state of our soil resources is an essential component to managing this resource. The use of hyperspectral data in soil erosion investigations is becoming more common due to its narrow, contiguous spectral bands, which are able to discern key soil minerals. The EnMAP satellite sensor builds on the foundation set by airborne hyperspectral imagers by providing high-spatial coverage and frequent temporal coverage.

The methods used for this analysis can be separated into three major sections. Firstly, the pre-processing of the AISA Eagle and Hawk hyperspectral data; secondly, the simulation of the EnMAP data; and lastly, the processing and analysis resulting in the final soil erosion state map. Besides the AISA Eagle and AISA Hawk hyperspectral data, Leica ALS50 (II) airborne laser scanner data and field data of the Camarena study site in the province of Toledo, Spain were used in this study. Figure 3.1 shows an image of the location of Camarena study site in relation to Madrid. 


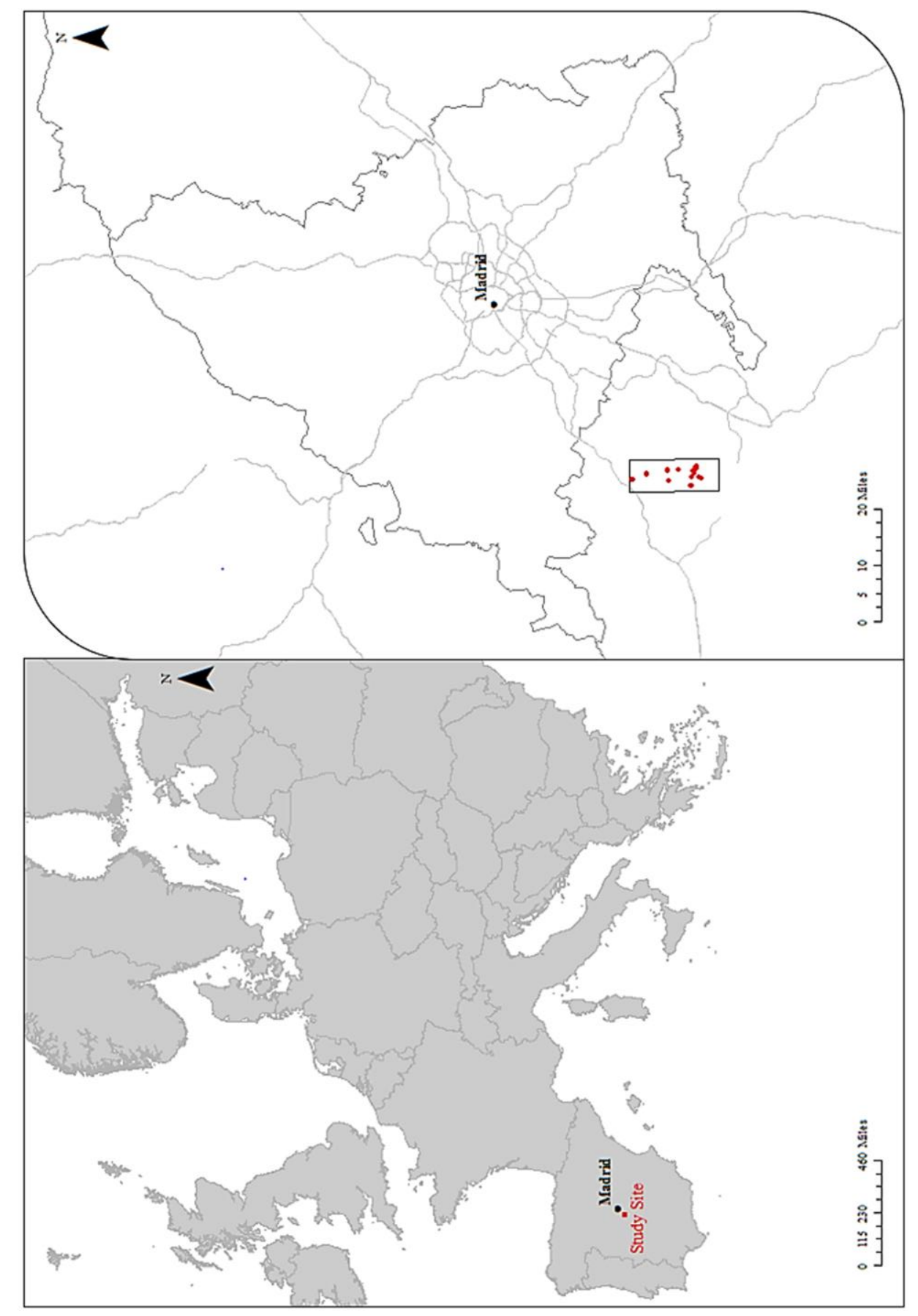

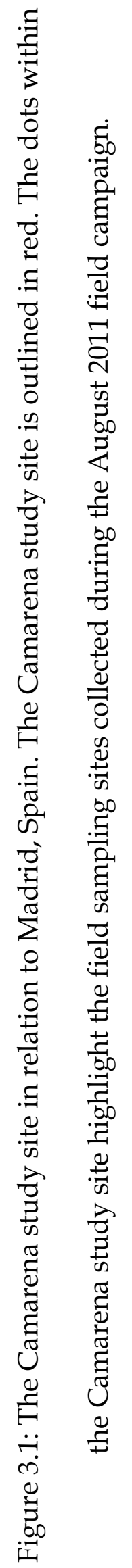


The AISA sensor was flown by the National Environment Research Council (NERC) - Airborne Research and Survey Facility (ARSF) based in Plymouth,

England. The Camarena study site is quite large and, for this reason, was subset after the completion of the pre-processing and simulation from its original 114$\mathrm{km}^{2}$ area, to a smaller $0.978-\mathrm{km}^{2}$ area covering only the main Southern field site (SU).

\subsection{Study Area}

The study area is located in the center of Spain, approximately $50 \mathrm{~km}$

Southwest of Madrid. The location is in the Northwest sector of the Autonomous Community of Castilla - La Mancha, Provence of Toledo (Table 3.1).

Table 3.1: Geographic coordinates (Zone 30N, ERTS 89 datum) for the Camarena study site.

\begin{tabular}{|c|c|c|}
\hline Waypoint & \multicolumn{2}{|c|}{ Geographic coordinates } \\
\hline & Latitude & Longitude \\
\hline Top left & $40^{\circ} 9^{\prime} 0.62^{\prime \prime}$ & $4^{\circ} 10^{\prime} 8.33^{\prime \prime}$ \\
\hline Top right & $40^{\circ} 9^{\prime} 4.14^{\prime \prime}$ & $4^{\circ} 4^{\prime} 3.54^{\prime \prime}$ \\
\hline Bottom right & $40^{\circ} 0^{\prime} 58.35^{\prime \prime}$ & $4^{\circ} 3^{\prime} 55.96^{\prime \prime}$ \\
\hline Bottom left & $40^{\circ} 0^{\prime} 54.85^{\prime \prime}$ & $4^{\circ} 10^{\prime} 0.03^{\prime \prime}$ \\
\hline
\end{tabular}


The site contains the town of Camarena and is a primarily agricultural region. Traditional agricultural activities within the study area include rain-fed crop cultivation, vineyards and olive groves.

The study area has a Mediterranean climate with a continental variant, which is characterized by cooler winter temperatures with low precipitation and dry summers. Precipitation occurs mostly in the autumn and spring, particularly in the months of November and April (World Weather and Climate Information, 2013). The average annual precipitation is $429 \mathrm{~mm}$ and the average annual temperature is $14.6^{\circ} \mathrm{C}$.

The dominant soils in the study area are highly developed alfisols and luvisols. Alfisols are typical to semi-arid to humid areas. Their subsoil is rich in clay and has relatively high fertility. Luvisols develop in climates that range from cold temperate to warm Mediterranean and typically in forests. They are characterized by a textual contrast between the A and B horizon, mainly a surface accumulation of humus, which overlies a layer that lacks clay and ironbearing minerals.

Soil horizons that are highly calcic (horizons in which $\mathrm{CaCO}_{3}$ has accumulated) are typically found in dry climatic regions such as the Camarena study area. Calcic haploxeralfs are the dominant alfisols present in the study area 
(Soil Survey Staff, 2010). Haploxeralfs are a large group under the alfisol soil order. Calcic haploxeralfs have an argillic or kandic horizon meaning that they are predominantly composed of clay or kaolinitic clay-like mineral. They are also characterized by a calcic horizon that has its upper boundary within $100 \mathrm{~cm}$ of the mineral soil surface.

The predominant luvisol in the study area is the calcic luvisol. It is commonly found in Mediterranean climates on flat or gently sloping land. Similar to the calcic haploxeralfs, they are also heavily clay enriched. They contain a calcic horizon between $50 \mathrm{~cm}$ and $100 \mathrm{~cm}$ from the soil surface. Typically, due to their structure, luvisols on steep slopes require erosion control measures (IUSS Working Group WRB, 2006).

The typical soil profile of the study area is composed of the A horizon, characterized by moderate organic matter content and coarse texture, the B horizon, characterized by fine texture and Fe oxides, and the $\mathrm{C}$ horizon characterized by high carbonate concentrations and coarse texture. Provided in Figure 3.2 is an example of a soil profile. The A horizon described here is the area of maximum accumulation of humus and has the greatest exposure to soil forming processes. The B horizon is the maximum zone of accumulation and alteration, while the $\mathrm{C}$ horizon is the zone of minimal accumulation and alteration (Agriculture Canada Expert System on Soil Survey, 1987). 


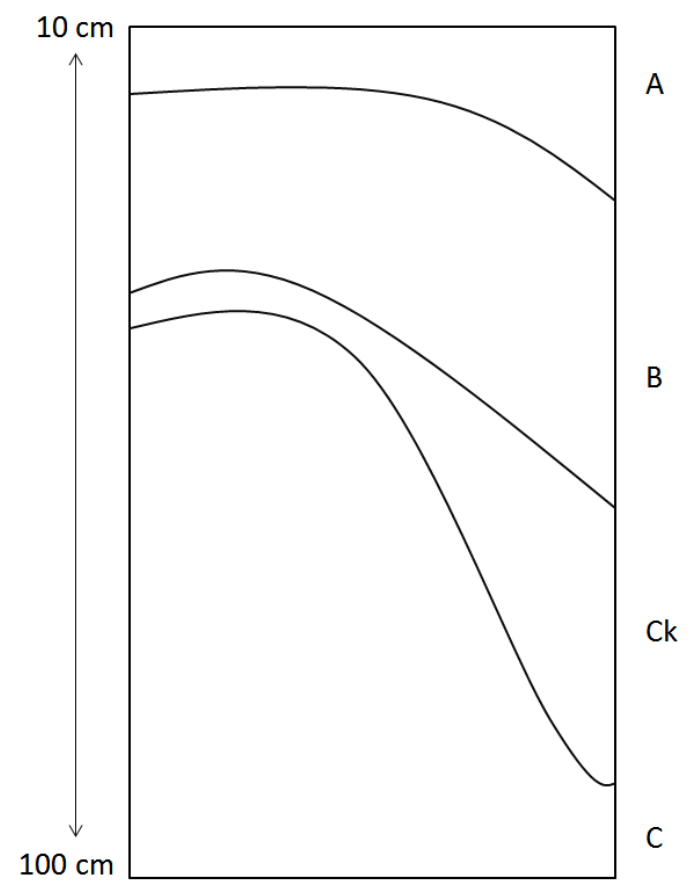

Figure 3.2: A typical soil profile in the study with associated soil characteristics. The A hoizon is characterized by moderate organic material content and coarse texture. The $\mathrm{B}$ horizon is fine textured and is rich in Fe oxides. The $\mathrm{Ck}$ horizon is rich in carbonates and coarse textured. The $\mathrm{C}$ horizon is composed of arkosic rock.

The location of the study site was chosen because it encompasses all of the desired requirements for the SEDMEDHY Transnational Access Project. They included: Mediterranean climate, extended agricultural rain-fed uses, and evolved soils and erosion features associated to contrasting soil horizons. 


\subsection{Data Collection}

\subsubsection{Airborne Data Collection}

Airborne hyperspectral and laser scanner data were collected for the Camerana study area. The flight was conducted by ARSF on August 8th $^{\text {th }} 2011$. A summer flight was chosen, because it provided a compromise between ideal soil properties unaffected by the dry season and increased soil surface exposure due to reduced photosynthetic activity (EUFAR, 2010). The summer flight was also chosen, because the summer months offer increased weather stability. The data acquisition was carried under clear sky and stable, dry conditions. The overlap between flight lines is approximately $20 \%$ for all flight lines. The duration of the flight lasted $58 \mathrm{~min}$ and, in that time, the solar azimuth and zenith angles changed considerably.

The hyperspectral data were collected with the combined AISA Eagle VNIR and AISA Hawk SWIR airborne hyperspectral sensors. The LiDAR (digital terrain) data was collected with the ALS50 (II) airborne laser scanner. A total of eight flight lines flown at an altitude of $5000 \mathrm{~m}$ were collected for the study area. The first and last flight lines of the acquisition were taken perpendicular to the main flight lines to provide additional information on changes due to illumination variation and the bidirectional reflectance properties of the surface. Figure 3.3 shows an initial mosaic of the six along-track flight lines and two across-track flight lines covering the Camerana study site. 


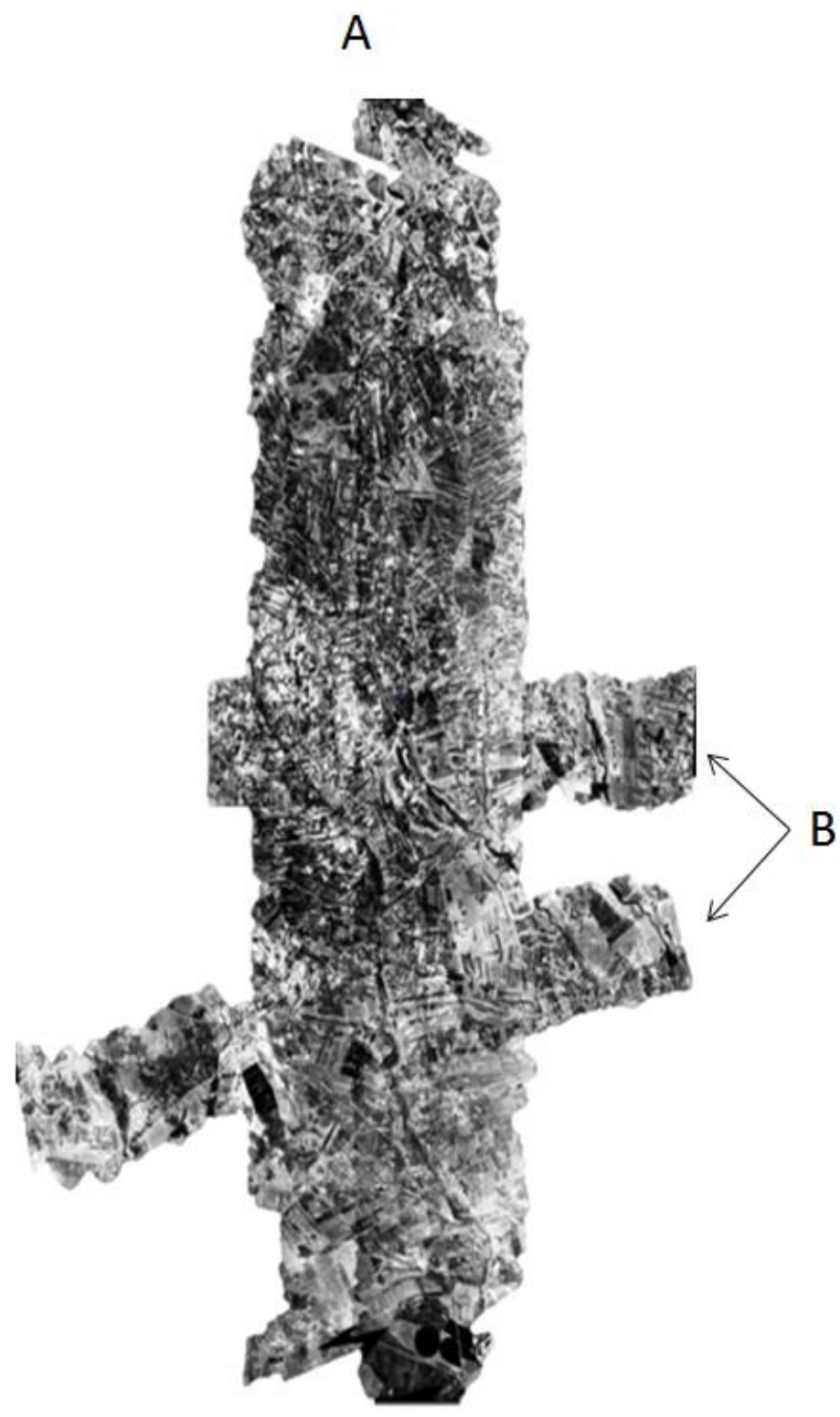

Figure 3.3: A preliminary mosaic of the eight AISA flight lines acquired on August 8th, 2011. A = Main flight lines; B = Perpendicular flight lines. 
The characteristics of the data collected by the AISA Eagle and AISA Hawk hyperspectral sensors, and from the LiDAR laser scanner are outlined in Tables 3.2 and 3.3, respectively:

Table 3.2: Characteristics of the AISA Eagle and AISA Hawk data. SSI = Spectral Sampling Interval, FOV = Field Of View

\begin{tabular}{|c|c|c|c|c|c|c|}
\hline$\underline{\text { Sensor }}$ & $\begin{array}{l}\text { Spectral } \\
\text { Coverage }\end{array}$ & $\underline{\text { Bands }}$ & $\underline{\text { SSI }}$ & $\underset{\text { Pixel Sizes }}{\text { Ground }}$ & $\begin{array}{c}\text { Spectral } \\
\text { Resolution }\end{array}$ & $\underline{\text { FOV }}$ \\
\hline $\begin{array}{l}\text { AISA } \\
\text { Eagle }\end{array}$ & $\begin{array}{c}400-1000 \\
\mathrm{~nm}\end{array}$ & 244 & $2.3 \mathrm{~nm}$ & $3 \mathrm{~m}$ & $2.9 \mathrm{~nm}$ & $37.7^{\circ}$ \\
\hline $\begin{array}{l}\text { AISA } \\
\text { Hawk }\end{array}$ & $\begin{array}{c}1000-2400 \\
\mathrm{~nm}\end{array}$ & 254 & $6.3 \mathrm{~nm}$ & $6 \mathrm{~m}$ & $8.0 \mathrm{~nm}$ & $24.0^{\circ}$ \\
\hline
\end{tabular}

Table 3.3: Characteristics of the Leica ALS50 (II) LiDAR data.

\begin{tabular}{|c|c|c|}
\hline Laser Scanner & $\underline{\text { FOV }}$ & Characteristics \\
\hline Leica ALS50 (II) & $40.0^{\circ}$ & $\begin{array}{c}\text { Multi-return height and } \\
\text { intensity signals }\end{array}$ \\
\hline
\end{tabular}

The data collected by the Leica ALS50 (II) laser scanner has an average point spacing of $1.5 \mathrm{~m}$. The elevation differences in the LiDAR data between overlapping flight lines are within the range of $4 \mathrm{~cm}$ to $52 \mathrm{~cm}$. A total of four data returns were recorded with $90 \%$ of the data recorded coming from the first return. A larger number of second returns were recorded in the vineyards and olive groves. 
The quality of the data collected by the combined AISA Eagle and Hawk sensors was evaluated by the ARSF and was satisfactory to good with striping found in the 1002-nm to 1154-nm range.

\subsubsection{Field Data Collection}

The field data was acquired between August 8th and 11th, 2011. It consisted of field spectroscopy, soil and vegetation sampling, estimation of vegetation residue coverage, and in-situ evaluation of soil conditions and horizons. Additionally, samples collected in the field were analysed in the laboratory to measure chemical and physical soil properties and to collect controlled spectral measurements. Measurements of the soil properties collected from the SU field site as presented in Table $3.4(\% \mathrm{w} / \mathrm{w}=$ Percentage weight/weight (grams of solute in $100 \mathrm{~g}$ solution), $\mathrm{YR}=$ Red-yellow.). 


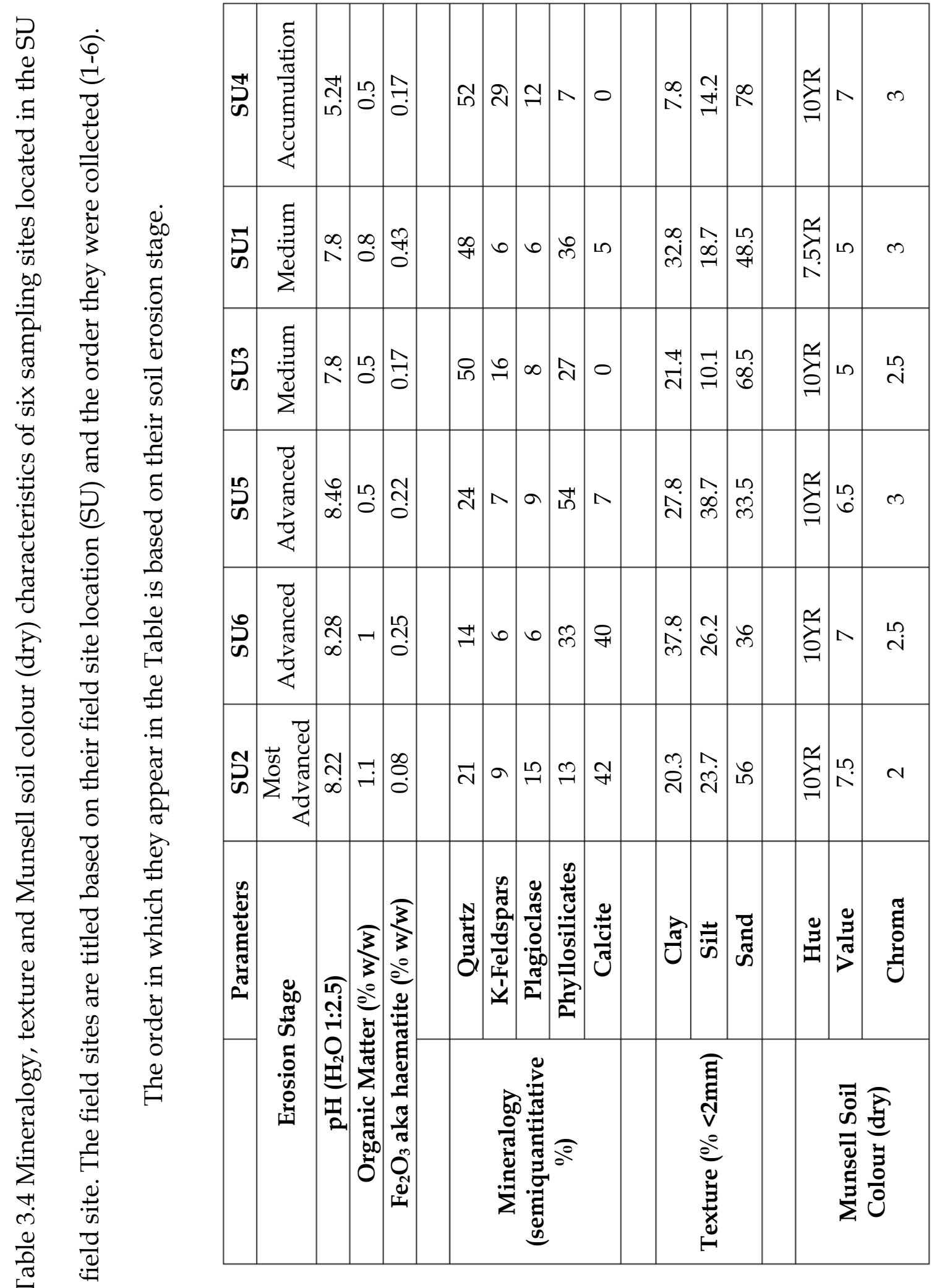


The field spectra were collected using an Analytical Spectral Devices (ASD) FieldSpec 3 full-range spectroradiometer and an ASD FieldSpec Pro spectroradiometer. The two spectroradiometers were cross-calibrated prior to taking field measurements. The measurements collected using these instruments cover the VNIR (350 nm - $1000 \mathrm{~nm})$ and SWIR (1000 nm - $2500 \mathrm{~nm})$ spectral range with a resolution of $3 \mathrm{~nm}$ and $10 \mathrm{~nm}$, respectively. The resulting field spectra contained a total of 2101 spectral bands.

The spectral measurements were collected hand-held, taken at nadir at arm's length from the operator and at one meter above the surface. Measurements were acquired on days with clear sky conditions at mid-day, between 11:00 and 15:00 when direct solar flux is at its highest. Care was taken by the field operator and the remaining crew and equipment to avoid any sources of shadowing near the measurement locations. Spectralon reference panel measurements were taken prior to commencing measurements at each field site.

Two different sampling schemes were used to acquire spectral measurements to ensure a collection of measurements, which were representative of the conditions within the study site. The first sets of measurements taken were with the intention of gathering spectral information representative of the main land-use categories within the study area. The measurements were acquired at eight test sites representing the main land-use 
categories within the study area. These categories include fallow, rain-fed cultivation of cereal crops, vineyards, olive groves and tilled land with organic residue left on the soil surface. An example of each of these land covers is presented in Figure 3.4. The second set of measurements were taken along transects in bare soil with the intention of recording the spatial variability of the soil properties and relief characteristics. All measurements and samples collected were geolocated at the time of acquisition using GPS (Figure 3.1).

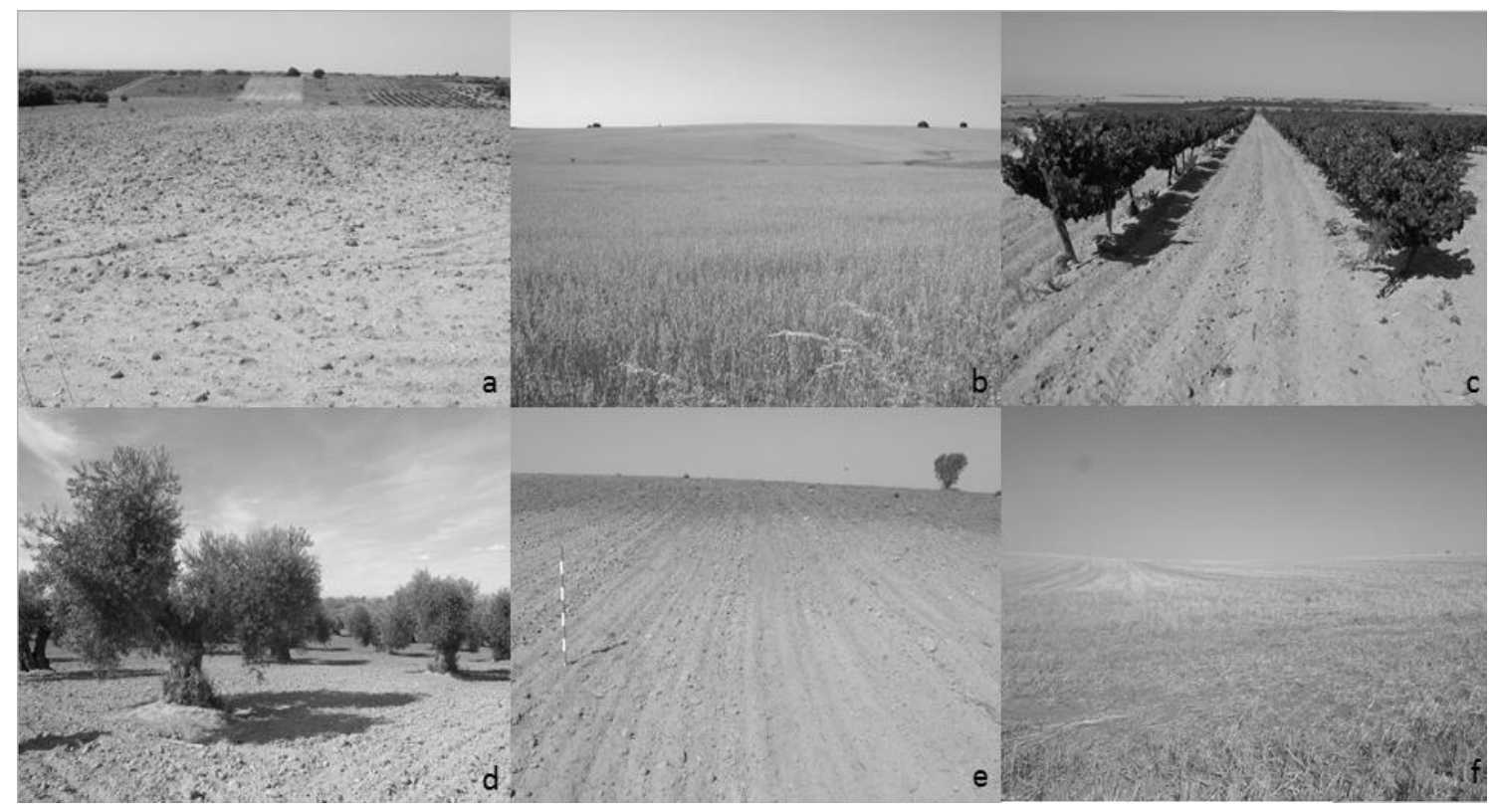

Figure 3.4: Images of the 8 surface covers used for field data collection. a) Fallow land. b) Rain-fed cereal cultivation. c) Vineyard. d) Tillage. e) Organic matter on surface. 
Each individual sample plot consisted of a central sampling point with three sampling points radiating outward each in the North, Southeast and Southwest directions. The first set of sampling points in the North, Southeast and Southwest directions were measured a distance of $3 \mathrm{~m}$ from the central sampling point. The second set of sampling points was measured out an additional $2 \mathrm{~m}$, for a total of $5 \mathrm{~m}$ from the central sampling point. Figure 3.5 demonstrates the sampling scheme for each site, including sub-plots based on the design presented for vegetation sampling by Schmidtlein et al. (2007).

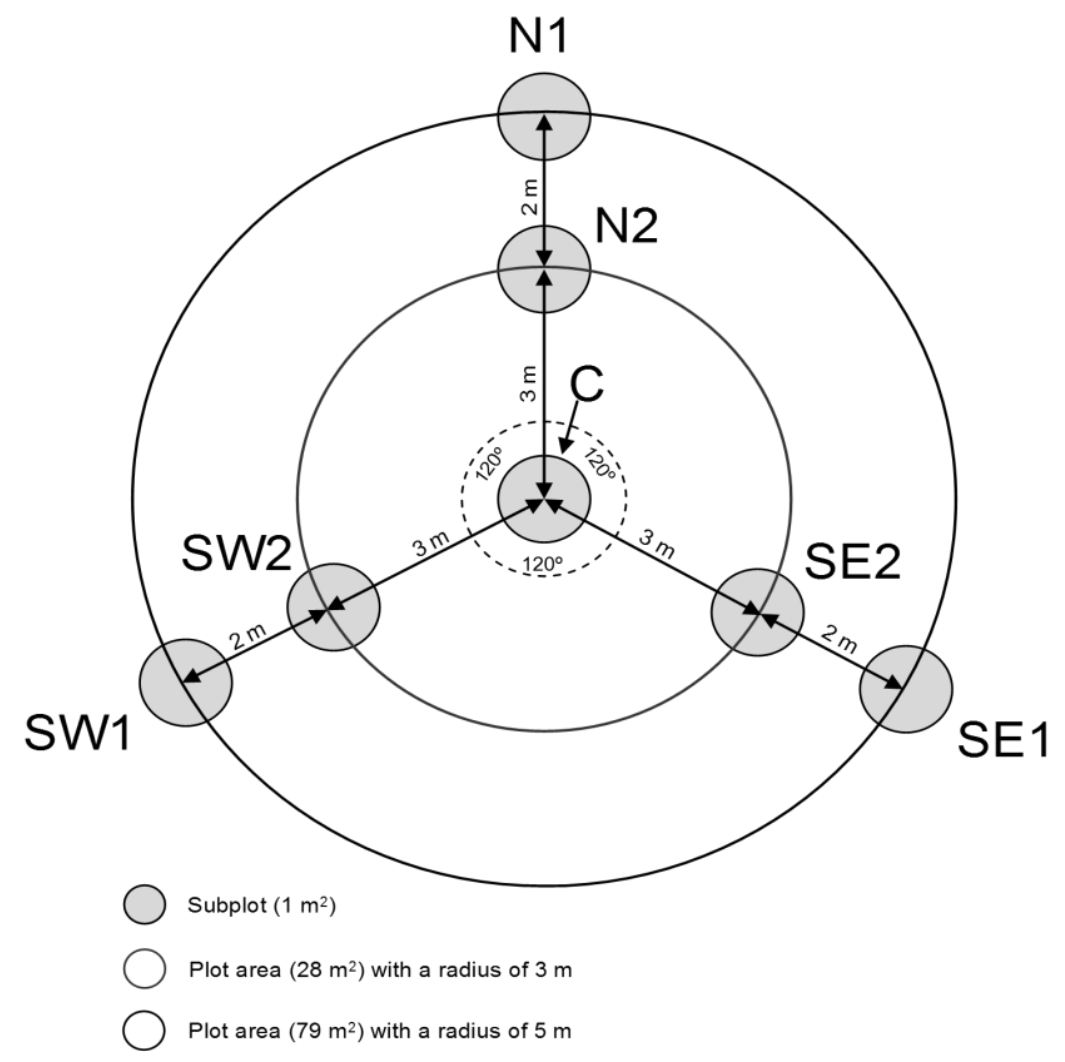

Figure 3.5: Sampling schematic used in the Camarena field site. 
The area covered by each sampling site was calculated as follows (Justice and Townshend, 1981):

$$
A=(P(1+2 G))^{2},
$$

where $A$ is the required sampling area $\left(\mathrm{m}^{2}\right), P$ is the pixel size $(\mathrm{m})$, and $G$ is the geometric accuracy (pixels). The area was calculated for pixel sizes of $2 \mathrm{~m}$ and 6 $m$ with an estimated geometric accuracy of one pixel.

\subsection{Hyperspectral Image Pre-processing}

Upon collection, image data may contain a variety of inconsistencies as a result of sensor, platform and environmental influences. These inconsistencies can range from the introduction of noise from the sensor system, geometric distortions as a result of platform movement, topography and the influence of atmospheric absorption and scattering on the reflected radiance. The goal of image pre-processing is to compensate for all these distortion in order to make the image resemble the natural scene as closely as possible.

The pre-processing of the AISA data included radiometric, geometric and atmospheric corrections as well as compensation for brightness variations, mosaicking and MNF (Figure 3.6). The final preprocessed ASIA data were then

used as input into EeteS to produce simulated EnMAP data. A soils classification 
of the preprocessed AISA data was also used for validation of the EnMAP classification results.

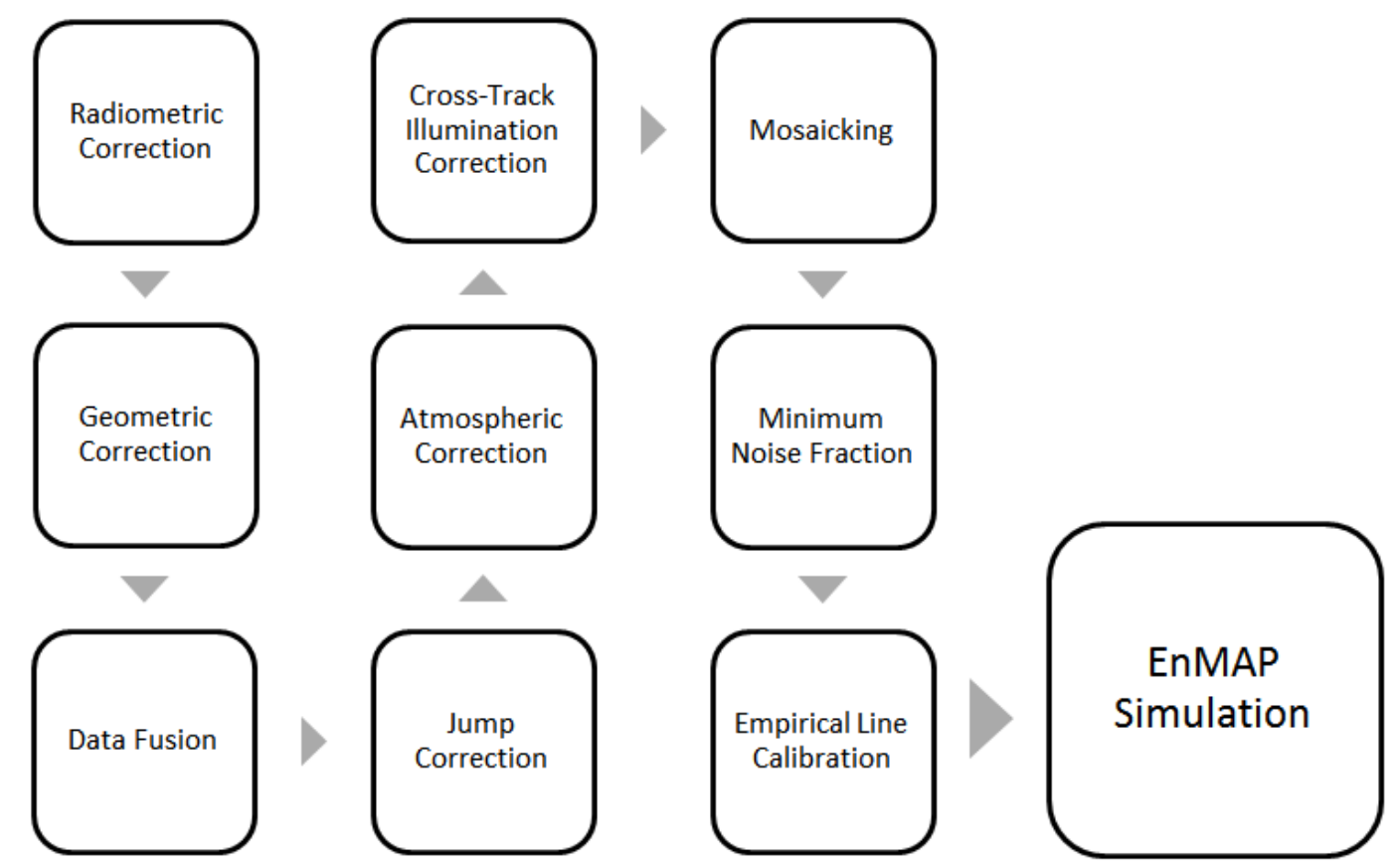

Figure 3.6: Flow chart of the preprocessing steps performed on the AISA Eagle and AISA Hawk data.

\subsubsection{Radiometric Calibration}

Radiometric calibration is designed to remove errors and inconsistencies in image DN's by providing a common physical scale (Teillet and Coburn, 2010). The ARSF provided the AISA Eagle and AISA Hawk data in level $1 \mathrm{~b}$ format. This format level entails the application of radiometric correction algorithms to the AISA Eagle and Hawk data with the resulting data in units of measured radiance 
$\left(\mathrm{Wm}^{-2} \mathrm{sr}^{-1}\right)$. The radiance data still contained further sensor radiometric inconsistences in the form of vertical striping in the SWIR and an across-track non-uniformity gradient in both the Eagle and the Hawk data. The destriping was corrected using software developed at the GFZ.

\subsubsection{Destriping}

Striping artifacts are a form of noise that are prevalent with pushbroom sensors and are the result of uncertainties and variation in the calibration of the detector elements (Rogass et al., 2011; Tsai and Chen, 2008). The stripes are created when an element in the detector produces no signal or is miscalibrated, which results in a line of no signal or miscalibrated pixels in the 2-D image.

All bands within the six flight-line images acquired with both the AISA Eagle and AISA Hawk were examined for striping. No striping was found in the AISA Eagle datasets. Sporadic and non-uniform vertical striping was found in the AISA Hawk dataset as well as periodic column drop-outs. Figure 3.7 shows an example of the vertical striping that was present. 


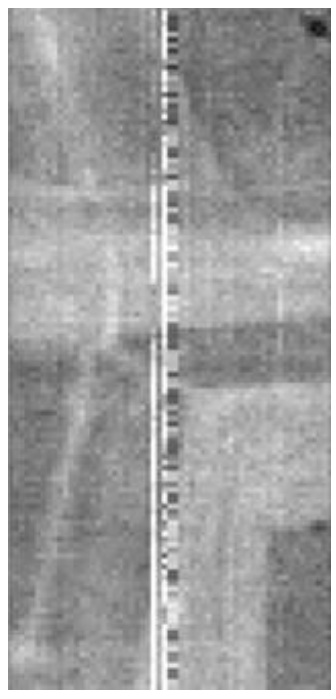

Figure 3.7: An example of the vertical striping found in the AISA Hawk data. The striping shown is inconsistent in magnitude along the column. This is representative of all of the striping, excluding the dropped columns, in the AISA Hawk image.

The destriping of the AISA Hawk hyperspectral data was conducted using an automatic detection of the linear features. Once all of the linear features were detected, either a recalibration or an interpolation correction was applied. The automatic detection of line features used differencing of adjacent bands to accentuate and locate the line features. To identify the striping features, a $1 \times 3$ window process the entire image, identifying the middle pixel as corresponding to one of four categories, local maxima (A), local minima (B), left linear increase (C) or right linear increase (D). These features can be seen in Figure 3.8. A 
histogram was then calculated for each image column identifying how often each of the four striping features occurred.

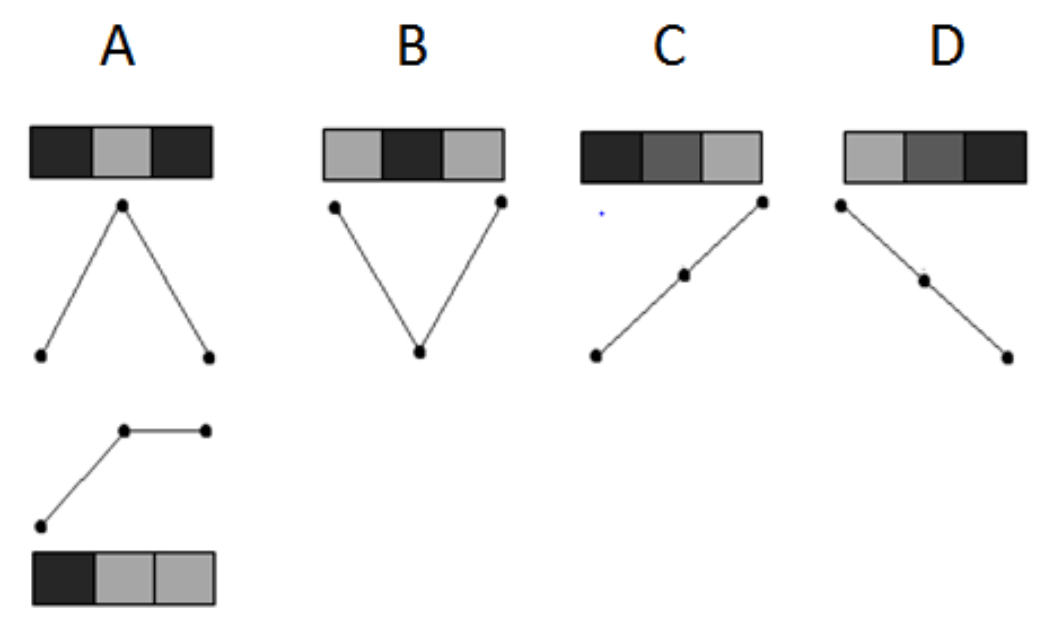

Figure 3.8: Shows the four types of striping features identified in the destriping process. From left to right: local maxima (top and bottom) (A), local minima (B), left linear increase (C), right linear increase (D).

The maximum occurrence was calculated for each column. For the column to be flagged as a striping feature, the maximum occurrence must exceed a user specified percentage, which for this research was set to a $60 \%$ threshold. The threshold value represents the percentage of the pixels in a column that were identified as a striping feature. Initially, the destriping was conducted using a $40 \%$ threshold, meaning any column in which $40 \%$ or greater of its pixels are identified as a striping feature is flagged as a stripe. The $40 \%$ threshold was found to be too strong for the scene and would identify linear image features, 
such as field boundaries and lines of olive trees, as striping. The threshold value used for the final data product was $60 \%$. Although a $60 \%$ threshold value was not able to automatically detect all stripes in the image, it reduced the number of false positives. This value was collected through trial and error.

The $60 \%$ threshold was unable to identify all of the striping features because they were often both spectrally and spatially non-uniform. To account for this, a manual detection of the features was also conducted. For this step, every band in each flight line was manually examined and the remaining stripes were recorded and were reprocessed in the subsequent steps along with the previously identified stripes.

Once all of the features were identified, the second stage in the process was recalculating the pixel values for the erroneous detectors. The general assumption for the recalculation of the pixel values was that the radiometric correction for the striped column was incorrect and as a result, can be corrected by a linear function using values from selected reference columns (Horn and Woodham, 1978). This in turn allows for the original data collected within those pixels to be preserved and only a moderate re-calibration to be applied to them instead of the removal of the data through full interpolation.

The last stage of the process was to interpolate the detector stripes with no signal. A spline interpolation was used to correct these stripes (Tsai \& Chen, 2008). The validation of the destriping was done visually. Individual bands in 
each flight line were examined for any uncorrected striping as well as for any false positives or spatial aberrations left as a result of the destriping.

\subsubsection{Geometric Correction}

The AISA Eagle and Hawk images for each flight line were geometrically corrected separately using software developed in-house specifically for this platform. It is customary to conduct the geometric correction after the atmospheric correction due to the resampling of the data that occurs in the orthorectification process. However, the procedure was reversed in this instance due to the additional files that were needed for the atmospheric correction. The software performed an orthorectification on each of the flight lines.

The coordinate transformation calculated the height and position of each pixel in the image and produced the raw scan angle file. The collinearity equation was used to determine the $X$ and $Y$ positions of the pixels. This equation is a physical model representing the geometry between the sensor projection center, the map coordinates and the image coordinates (Konecny, 1972). The raw scan-angle file contains the scan angles for each of the detectors. These angles were calculated for each pixel using the FOV and the pixel location within the FOV.

Nearest neighbour resampling was used to produce the geocoded image. This type of resampling was chosen over bilinear interpolation or cubic 
convolution, because the original pixel values are maintained, preserving the spectral integrity of the data (Schlapfer et al., 2001).

The DEM and the scan angle files were resampled to match the spatial characteristics of the AISA Hawk data and were output as ATCOR-4 compatible files. The orthorectified AISA Eagle and AISA Hawk flight lines from this procedure were then fused to converge the two spectral ranges into a single image that cover the full spectral range from $400 \mathrm{~nm}$ to $2400 \mathrm{~nm}$.

Typically with the use of ATCOR-4 for the atmospheric correction, PARGE would have been used to generate the required scan angle files and additional ATCOR compatible output files (Schläpfer et al., 1998). However, the software was unavailable for this research.

\subsubsection{Data Fusion}

The data fusion was also conducted using in-house software. For each flight line, the last band of the AISA Eagle data $(998.46 \mathrm{~nm})$ was fused to the first band of the AISA Hawk data $(1002.3 \mathrm{~nm})$. The procedure consisted of establishing a spatial relationship between the AISA Eagle and AISA Hawk data by locating the center location of the Eagle pixels on the Hawk image. A predefined Gaussian filter, including the sensor Point Spread Function (PSF), was then used to resample the AISA Eagle data to the spatial resolution of the AISA Hawk. Finally, as a result of the two different FOVs of the sensors, the AISA Eagle data needed to be cropped to fit the spatial extent of the AISA Hawk data. 


\subsubsection{Jump Correction}

A jump can be described as a spectral offset resulting in different radiance values between adjacent spectral bands. A spectral jump was detected in the spectra of the combined AISA Eagle and Hawk data as a result of the data fusion process. The jump introduced was an irregularity and was not actually in association with any surface cover within the image. Because the jump did not have a physical meaning, it needed to be corrected for in the image.

The procedure consisted of 1) identifying the jump characteristics, such as magnitude and extent; 2) spectrally unmixing the scene using a predefined spectral library of scene surface covers; 3 ) generating an "ideal" (without a spectral jump) spectrum for each pixel in the image by mixing the spectra in the spectral library using the surface cover fractions estimated for each pixel in step 2 ; and 4) replacing the section of the spectrum affected by the jump for each pixel in the image with the "ideal" spectrum generated for each corresponding pixel.

The jump was inconsistent in size and shape but occurred consistently in the same spectral location, at approximately $1000 \mathrm{~nm}$. Official documents from the ARSF noted that the data collected using the AISA Eagle and AISA Hawk sensors were not reliable above $900 \mathrm{~nm}$ and below 1100nm, respectively (ARSF, 2011). The characteristics of the jump were determined by comparing the radiance image spectra to geolocated reflectance field spectra. Because the jump correction was performed prior to the atmospheric correction, the reflectance 
field spectra were converted into radiance using an in-house atmospheric RT software. By comparing the image radiance spectra to the converted field radiance spectra, the extent of the affected wavelengths was determined to be between $978 \mathrm{~nm}$ and $1096 \mathrm{~nm}$.

Figure 3.9 shows a comparison between the radiance signatures gathered from the image and radiance signatures converted from field reflectance spectra for two field locations within the Camerana study site. The radiance spectra have been smoothed to remove a majority of the noise so that the jump can be more clearly visible.

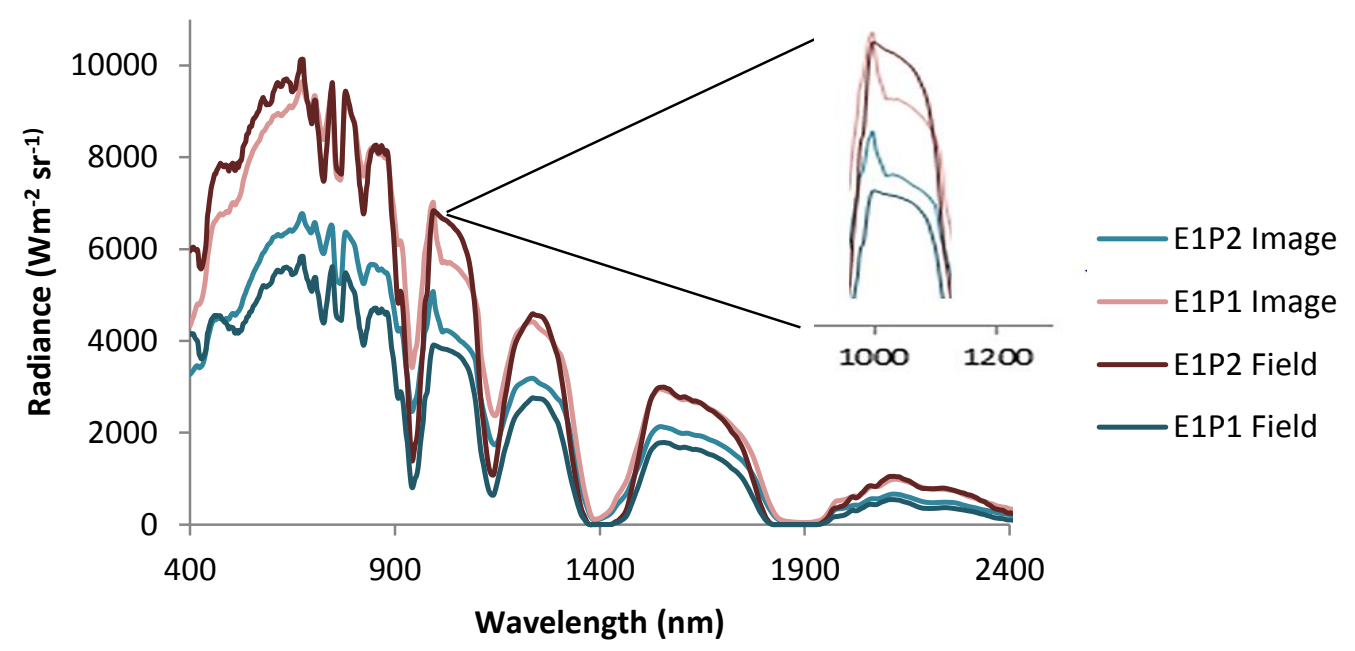

Figure 3.9: Two field location spectra converted to radiance and the corresponding image radiance spectra used to define the jump characteristics. The jump is located between $978 \mathrm{~nm}$ and $1096 \mathrm{~nm}$. This is where the image and field radiance spectra differ greatly. This is the wavelength range where the image spectra were corrected. 
The jump correction was conducted using unconstrained spectral unmixing/mixing techniques. It should be noted that an additional offset term $\left(X_{0}\right)$ was included into the linear spectral mixture equation to allow for shifting of the spectral signature about the $\mathrm{Y}$ axis to account for variations in radiance intensity. The linear spectral mixing model applied in the jump correction is as follows:

$S_{i j}=X_{0}+X_{1} * S_{1}+X_{2} * S_{2}+X_{2} \ldots+w$,

where $X_{0}$ is the additional term accounting for variation in the $Y$-axis.

A spectral library was constructed using ASD spectra of various surface covers from both the Camerena study site and also from an additional Spanish study site. The spectral library was composed of two green vegetation, two mixed brown/green vegetation, two brown vegetation, one badlands, one riverbed, one pavement, three water and four soil spectra. The four soil spectra were collected from the Camarena site and accounted for the spectral variability in soil surface cover within the scene.

The spectral library was converted from reflectance into radiance using the same in-house RT software as before. Since water vapour content was not measured in the field and had not yet been estimated using an atmospheric modeling software, the spectral library was converted to radiance eight times, 
each time with different water vapour content. These spectra were then resampled to the spectral resolution of the combined AISA Eagle and Hawk data. The optimal spectral library for the unmixing procedure was chosen based on the atmospheric water absorption band depth. The library with an absorption depth, which most closely resembled that of the image, was chosen for the unmixing procedure.

Once the image had been unmixed, the same spectral library was then mixed based on the fraction values estimated in the unmixing to simulate an artificial spectrum for each pixel in the image. This in turn generated an "ideal" spectrum, without a spectral offset feature, which was used to replace the original combined AISA Eagle and Hawk spectra within the defined jump region, between $978 \mathrm{~nm}$ and $1096 \mathrm{~nm}$. All regions of the spectra outside of the defined jump region were not altered.

\subsubsection{Atmospheric Correction}

Atmospheric correction compensates for the unwanted scattering and absorption effects caused by the atmosphere in remotely sensed data. Atmospheric correction is a particularly important pre-processing step when the data is going to be used in the extraction of physical parameters or when it will be used in variable temporal or spatial studies (Haboudane, et al., 2002; Thiemann and Kaufmann, 2002; Song et al., 2001). 
The atmospheric correction was conducted using ATCOR-4's rugged terrain option. Compared to the flat terrain option, this option includes a DEM to account for topographical influences and illumination effects. A low-pass filter was used to smooth the resampled DEM files generated in at the time of the geometric correction. Such a filter was applied because the original DEM output created artifacts in the ATCOR reflectance results. Topographic calculations including slope/aspect, sky view and shadow were included in the atmospheric correction. Each flight line was atmospherically corrected separately to account for the differences in heading direction and solar geometry. Table 3.5 details the flight and solar characteristics at image acquisition, which were used as input parameters into ATCOR-4.

Table 3.5: Details of the flight and solar geometry parameters for the image acquisition. Flight lines 22001 and 22008 are the across-track flight lines. This can be seen by their heading directions in comparisons to the other flight lines.

\begin{tabular}{|l|l|l|l|l|l|}
\hline Flight Line & $\begin{array}{l}\text { Direction } \\
\text { (degrees) }\end{array}$ & $\begin{array}{l}\text { Altitude } \\
\text { (m) }\end{array}$ & Time & $\begin{array}{l}\text { Solar Azimuth } \\
\text { (degrees) }\end{array}$ & $\begin{array}{l}\text { Solar Zenith } \\
\text { (degrees) }\end{array}$ \\
\hline 22001 & 68 & 5085 & $14: 34$ & 239.6 & 37.3 \\
\hline 22002 & 347 & 5042 & $14: 44$ & 242.4 & 38.9 \\
\hline 22003 & 194 & 5043 & $14: 52$ & 244.5 & 40.3 \\
\hline 22004 & 346 & 5054 & $14: 59$ & 246.3 & 41.5 \\
\hline 22005 & 195 & 5030 & $15: 07$ & 248.2 & 42.9 \\
\hline 22006 & 345 & 5029 & $15: 15$ & 250 & 44.3 \\
\hline 22007 & 192 & 5036 & $15: 23$ & 251.8 & 45.8 \\
\hline 22008 & 269 & 5096 & $15: 32$ & 253.8 & 47.5 \\
\hline
\end{tabular}


A rural atmosphere with an estimated water vapour content of $1.05 \mathrm{~cm}$ and a flying altitude of $5000 \mathrm{~m}$ were chosen for the scene. A water vapour map was generated from the scene by ATCOR-4 using the $820 \mathrm{~nm}$ atmospheric water absorption feature (D. Schlapfer, personal communication, June, 2012). Visibility was estimated by ATCOR-4 as being $80 \mathrm{Km}$. Bands in the $760 \mathrm{~nm} \mathrm{O}_{2}$ region and the $725 / 825 \mathrm{~nm}, 940 / 1130 \mathrm{~nm}$ and $1400 / 1900 \mathrm{~nm}$ water vapour regions were interpolated. An empirical BRDF correction was also applied to the data to supress the brightness variation between/within the flight lines.

The atmospheric parameters used in the atmospheric correction remained the same for all flight lines. The final reflectance image still contained a substantial amount of noise and large BRDF effects and non-uniformity effects in across-track and along-track directions.

\subsubsection{Cross-Track Illumination Correction}

Each individual flight line exhibited decreasing brightness from one edge of the imagery to the other in the across-track direction as a result of sensor nonuniformity (Toivonen et al., 2006). Brightness variations also occurred between the different flight lines, which is a BRDF effect caused by the direction of the flight heading which alternated between the direction of the Sun and away from the Sun (Staenz et al., 1993 ). Lastly, there was a decrease in overall brightness in 
the along-track direction from the top of the scene to the bottom of the scene.

This is a topographical effect as a result of the overall decrease in elevation in the North/South direction within the scene. Influences such as the change in solar geometry, related to the long duration of the image acquisition (50 $\mathrm{min})$, and the direction of the flight heading were also believed to have an effect on the brightness variations within the scene. The change of solar position over the flight duration can be seen in Figure 3.10.

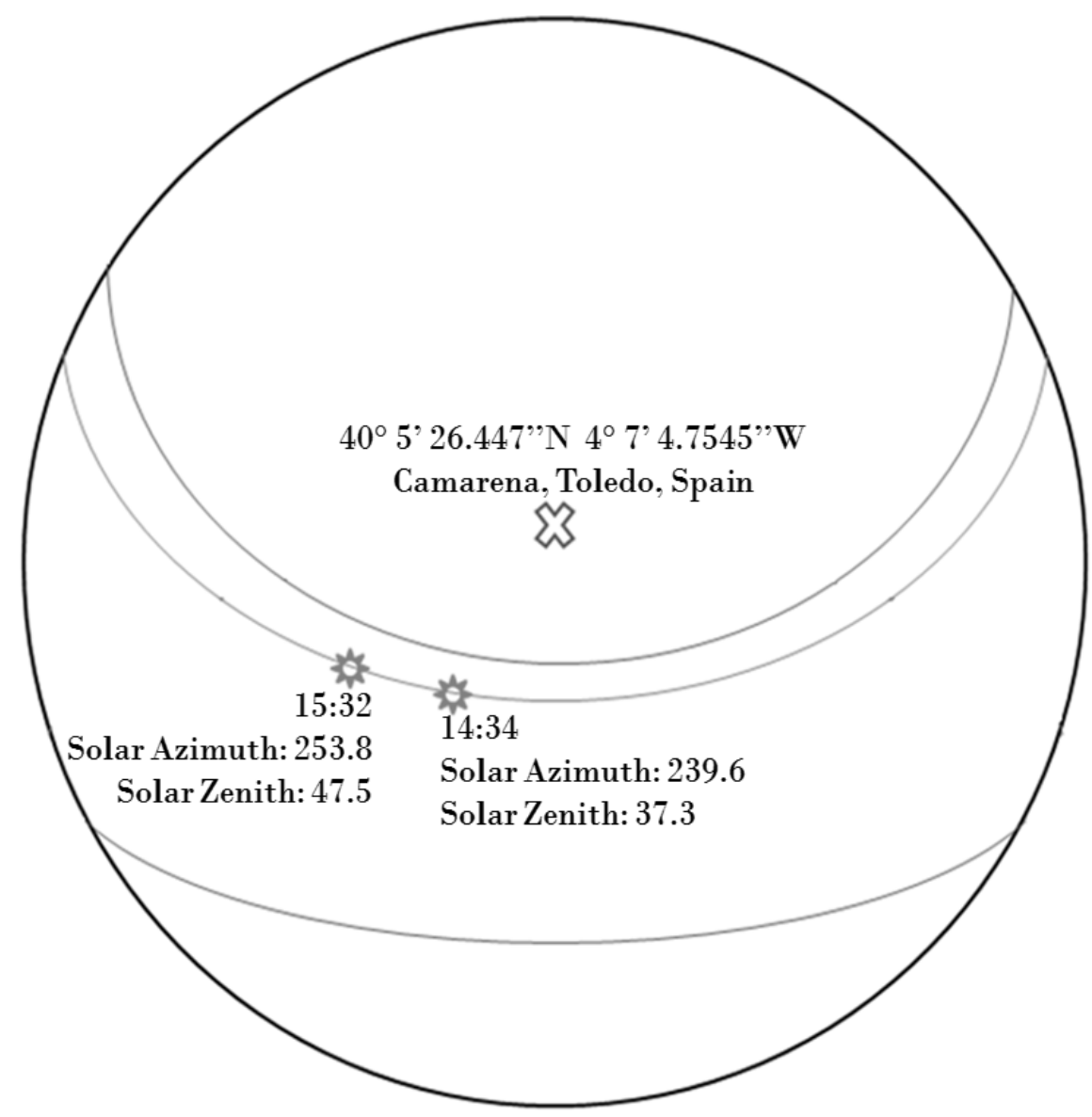

Figure 3.10: Change in sun position in relation to Camarena, Toledo, Spain over the duration of the flight campaign. 
Attempts were made to supress the brightness variations within each individual flight line, in both the across-track (non-uniformity) and along-track (topographical) directions using the cross-track illumination correction (Exelis, 2014a) available in the Environmental Visualization software (ENVI). This correction was applied to each individual flight line (Dadon et al., 2010).

The cross-track illumination correction was applied in the same manner in the across-track and along-track directions for all flight lines. It calculates the along-track mean values for each band and uses them to determine the mean illumination variation in the cross-track direction. The same is true when correcting for illumination difference in the along-track direction where the mean across-track values are calculated for each band and are used to determine the mean illumination variation in the along-track direction.

The correction is a polynomial function of a user-defined order, which is fit to the means and is used to supress the brightness variation. A multiplicative method with a first-order polynomial was chosen for the correction. This combination of correction method and degree of polynomial were selected, because it balanced the brightness differences better in both the across-track and along-track directions than any other combination of correction method and polynomial order. A first-order polynomial was also chosen for the correction over any higher polynomials, because increasing the polynomial could potentially negatively affect the entire scene by removing local variation in the 
data. This was evaluated by comparing horizontal and vertical profiles of the same location between all the combinations attempted.

The cross-track illumination correction was conducted following the atmospheric correction because of the possible interference it may have with certain aspects of the atmospheric correction. This was particularly a concern with parameters that are estimated directly from the data for the atmospheric correction, such as water vapour content or visibility.

\subsubsection{Mosaicking}

Because the footprint of the individual flight line images is smaller than the study area, the orthorectified images were compiled into an image mosaic. Prior to mosaicking the flight lines, they were resized to remove the majority of the overlapping edges. This was done to eliminate the strong spatial and spectral inconsistencies inherent in pushbroom sensors that become more prominent away from nadir. The overlap between flight lines ranged from 53 pixels up to 113 pixels, approximately $3.18 \mathrm{~km}$ to $6.78 \mathrm{~km}$, respectively. The resized flight lines are shown in a mosaic template in Figure 3.11. 


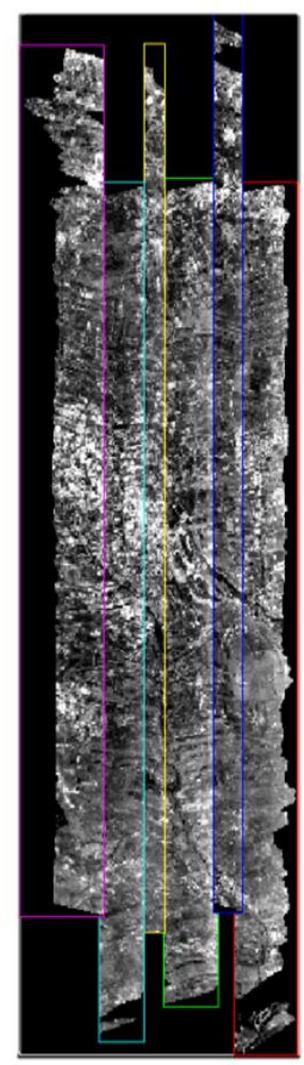

Figure 3.11: Flight line subsets used for the mosaicking of the Camarena study site.

The substantial brightness variations mentioned in the previous section became more evident along the seam lines where the flight lines were mosaicked together. Neither feathering nor colour balancing was applied to the mosaic to try to suppress these effects. The option to feather the image scene was omitted from the mosaicking, because it requires a very good geometric accuracy. This is because the feathering calculates an average of the overlapping pixels between flight lines. If the overlap is not accurate enough, the feathering will average adjacent pixels creating unreal pixel mixtures along the seam lines. The RMS error for the geometric correction ranged from $5.96 \mathrm{~m}$ to $12.04 \mathrm{~m}$, which would 
result in unnecessary mixing along the seam line and as a result the feathering was not included in the mosaic.

The colour balancing option was applied as a test to see if it could remove the drastic brightness differences along the seam lines, but there was unfortunately little to no improvement. The albedo differences between flight lines can be seen in Figure 3.12, with drastic differences visible in the green vegetation between flight lines 2 and 3 .
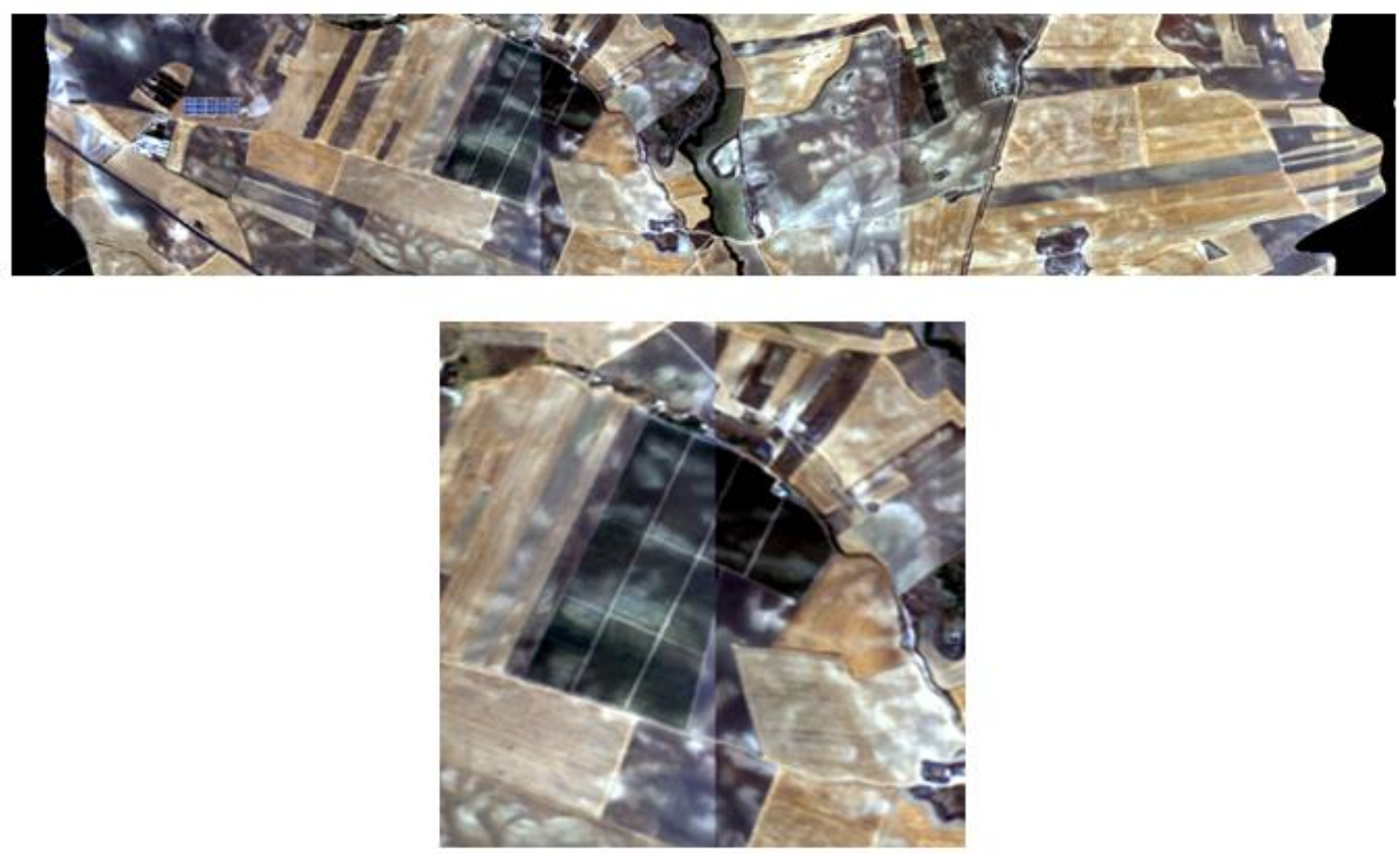

Figure 3.12: A horizontal subset of the mosaicked flight lines (top) and a close up on a seam line (bottom) in the image subset shown on the top. The close up highlights the brightness variation between the two flight lines. 


\subsubsection{Minimum Noise Fraction}

A Minimum Noise Fraction (MNF) analysis was applied to the mosaicked image. An MNF consists of two cascading Principal Component Analysis (PCAs) (Chen et al., 2003). MNF was conducted to segregate the noise component and ultimately reduce the dimensionality of the data (Green et al., 1988) before proceeding to the EnMAP simulation to maximise efficiency and improve the results of the subsequent processing (Tseng, 2000).

The number of coherent bands to be included in the inverse MNF was determined using a MNF normalization. The equation for the MNF normalization is as follows:

$$
\left(\frac{\partial}{\sum \partial}\right) * \mathrm{n}
$$

where $\partial$ represents the eigenvalue and $n$ is the number of output MNF images. Using this Equation, all eigenvalues with a normalization value above 1.00 are selected as coherent. The first 38 components out of a total of 486 bands had normalization values above 1.00 , which correspond to coherent image components. Accordingly, these first 38 bands were used in an inverse MNF to return back to the original dimensions of reflectance and wavelength, excluding incoherent components composed largely of noise. 


\subsubsection{Empirical Line Correction}

An Empirical Line Correction (ELC) was used to remove residual atmospheric absorption features in the data that remained after the atmospheric correction. The ELC was chosen over other smoothing methods such as the Empirical Flat Field Optimized Reflectance Transformation (EFFORT), because it was largely remnant atmospheric absorption features that needed to be removed in contrast to sensor noise. The ELC was conducted on the entire mosaic rather than on the individual flight lines because there were not enough field locations within each flight line to apply it individually. Correcting for the remnant atmospheric features is a critical issue since noisy reflectance products can render spectral-based classification products incorrectly (Goodenough et al., 2003).

The ELC uses linear regression for each band to calculate gain and offset values that are then applied to the data (Farrand et al., 1994). Figure 3.13 shows how the gain and offset terms are determined. The gain values are a multiplicative term and the offset values are an additive term. 


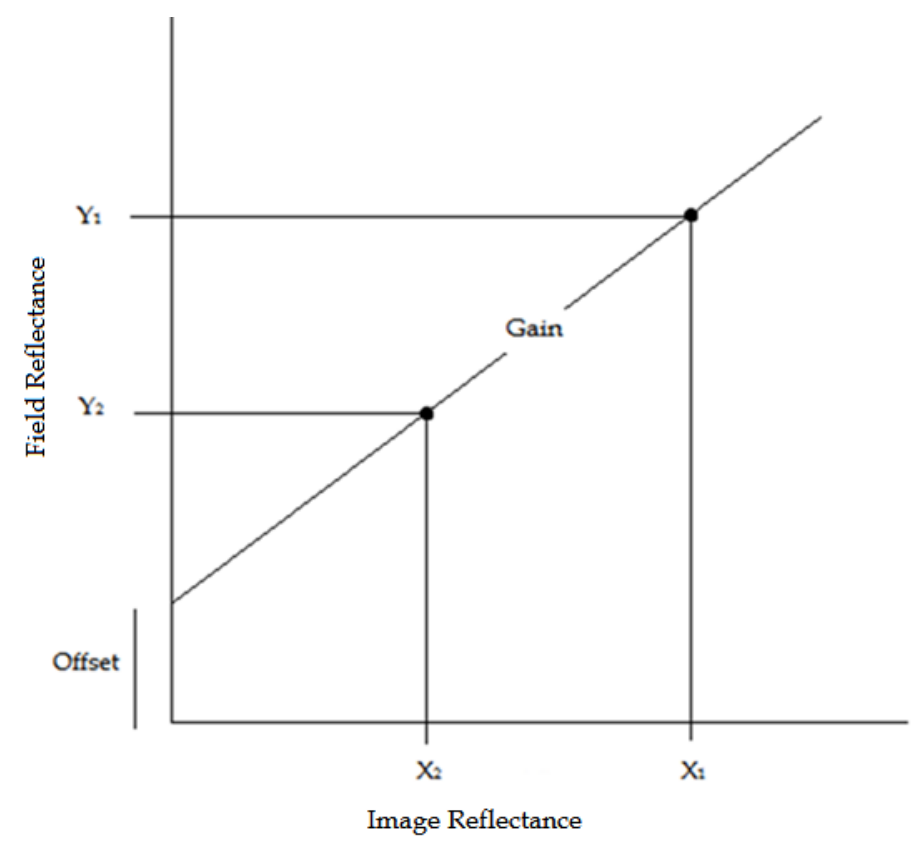

Figure 3.13: Linear regression used to derive the gain and offset coefficients for the ELC in one band. In this image, the points in the plot represent the spectra being used for the ELC. The point corresponding to $X_{1}, Y_{1}$ is a bright target and the one corresponding to $\mathrm{X}_{2}, \mathrm{Y}_{2}$ is a dark target. The positions of the points in the plot are determined by the value of the field spectra and the value of the corresponding image spectra in that particular band. The gain is calculated using

$$
\frac{Y_{1}-Y_{2}}{X_{1}-X_{2}} .
$$


The equation below shows the application of the gain and offset values to the image data (Smith \& Milton, 1999; Roberts et al., 1986) as follows:

$S_{k}=\rho_{k} A_{k}+B_{k}$

where $S_{k}$ is the output value (reflectance) for a pixel in band $k, \rho_{k}$ is the field surface reflectance in band $k, A_{k}$ is the gain term in band $\mathrm{k}$ and $B_{k}$ is the offset term in band $\mathrm{k}$.

A dark and a bright target are chosen as reference spectra for the correction to provide the most accurate linear regression. Including additional spectra also helps to improve the calibration. When more than one spectrum is used for the calibration, the regression for each band is calculated by fitting the regression line through all of the spectra.

Four spectra were used for the calibration, a burned soil, an iron and clayrich soil, a highly calcitic/clay mix soil and a very sandy soil. These four spectra were chosen for the correction, because they spread a wide range of reflectance values. Figure 3.14 shows the four field spectra used for the ELC. 


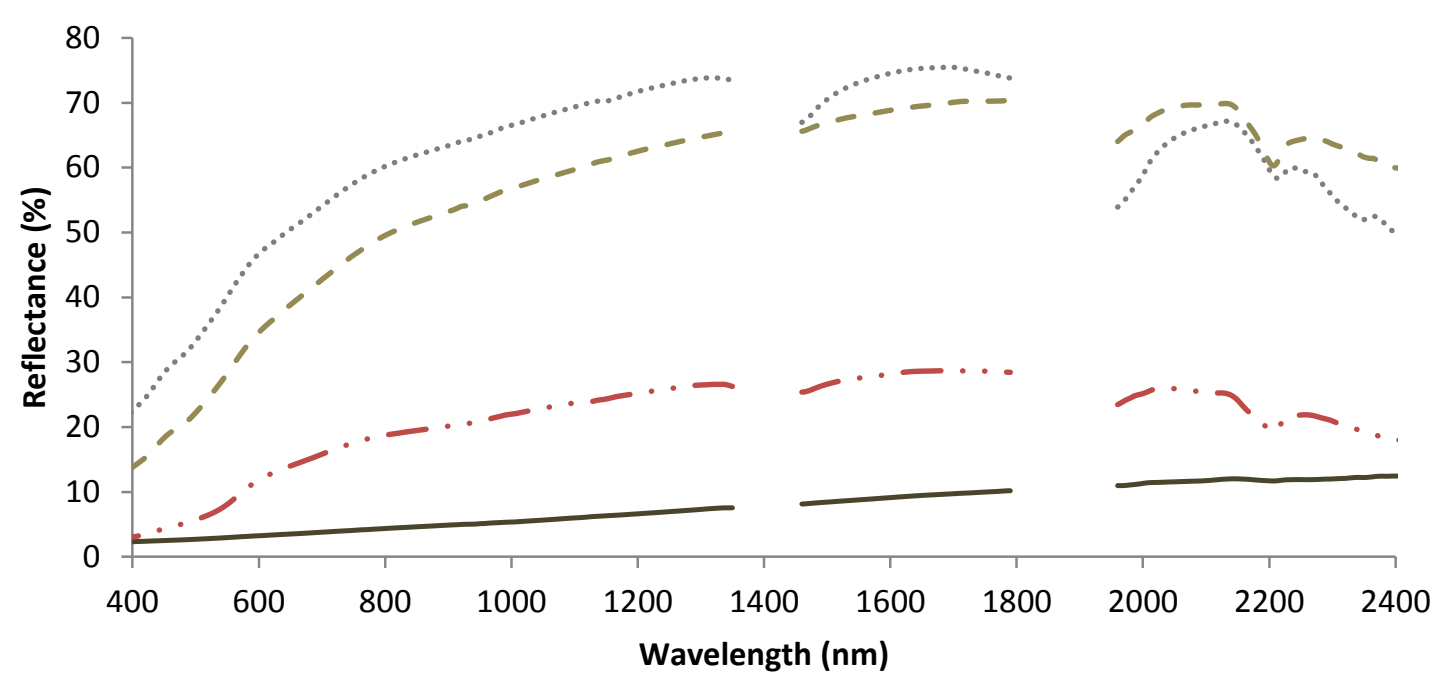

— Burned Soil _ —. Iron and Clay Rich Soil ........ Calcitic/Clay Mixture - - - Sandy Soil

Figure 3.14: Field spectra selected for the ELC of the image data.

The output AISA data from the ELC was included in the classification procedure and was used as the ground-reference image in the validation of the EnMAP results (Thomlinson et al., 1999).

\subsection{EnMAP Simulation}

The EnMAP data simulation was carried out using EeteS. The preprocessed combined AISA Eagle and Hawk reflectance data cube was used as the input for the EeteS simulation software (Segl et al., 2012). The simulation process is comprised of two separate components, the forward and backward simulation. The final result of EeteS is a simulated Level-2 (L-2), atmospherically and geometrically corrected EnMAP reflectance image cube (Segl et al., 2012). Figure 3.15 depicts the EeteS simulation process. The simulation was done for the Beginning of Life (BOL) of the EnMAP sensor. BOL characterises the expected 
imaging characteristics of the sensor in the initial stages of its launch. End of Life (EOL) simulation, in contrast, would include the expected degradation of the sensor components over its life span.

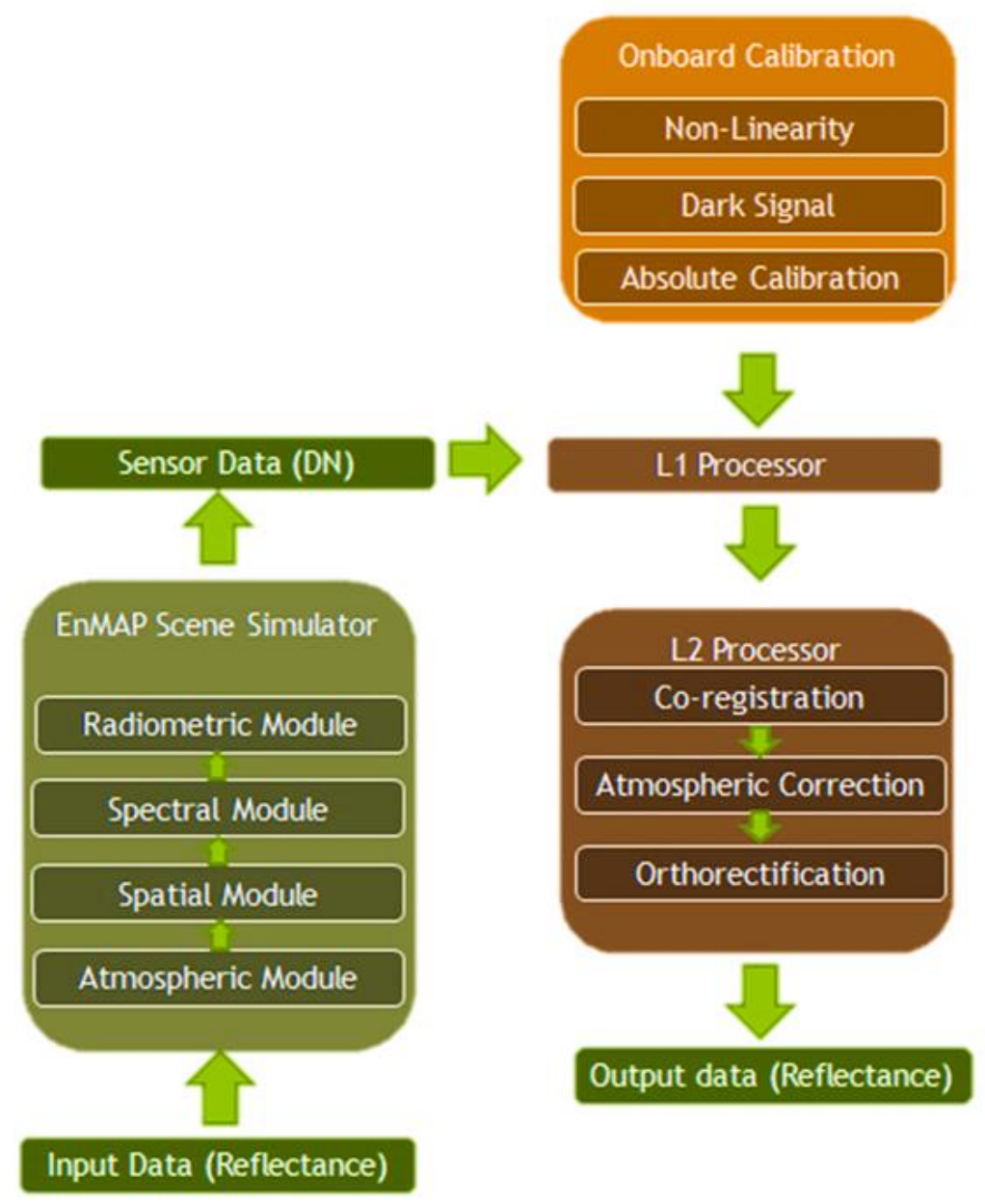

Figure 3.15: EeteS simulation process from input reflectance data to output EnMAP reflectance data. 


\subsubsection{Forward Simulation}

The forward simulation consists of four modules, atmospheric, spatial, spectral and radiometric. These four modules produced EnMAP DN values from the combined AISA Eagle and Hawk reflectance data cube.

\subsubsection{Atmospheric Module}

The first module in the forward simulation is the atmospheric module (Segl et al., 2012). The atmospheric module converts surface reflectance to TOA radiance data using atmospheric parameters estimated using the MODTRAN 4 radiative transfer code. The atmospheric module uses as input the day, month and Greenwich Mean Time (GMT) of the image acquisition, the latitude and longitude of the scene, selected aerosol and atmospheric model, and userdefined AOT (0.8) and CWV (systematically varied between 1.85-2.15 cm). No additional parameters, such as cloud cover or shadow, were used in the simulation (Segl et al., 2012).

\subsubsection{Spatial Module}

The spatial module is the second module in the forward simulation. It performs the spatial recoding of the image data and simulates spatial aberrations caused by the telescope optics, slit and curved prisms (Segl et al., 2010). The spatial module employs both the geometry model and the optical sensor model. The former is defined by the pointing vectors of all VNIR and SWIR detector elements in relation to the target. Considerations for the geometry module 
include the position, attitude, speed, heading and the off-nadir pointing capabilities of the sensor (Segl et al., 2010). The optical sensor module performs the spatial recording of the pixels by combining the spectral information with the specific PSF in the across-track direction (Guanter et al., 2009). The outcome of the spatial module is spatially resampled $30-\mathrm{m}$ radiance data cube.

\subsubsection{Spectral Module}

The third component of the forward simulation is the spectral module. The spectral module considers the Spectral Response Function (SRF) of each band, as well as spectral non-uniformities, such as spectral smile and spectral shift when performing the spectral resampling (Segl et al., 2012). The first part of this module defines the central wavelength location, SSI and the bandwidth at Full-Width Half-Maximum (FWHM). The simulation was done separately for each of the two detectors to account for differences in the spectral performance.

The VNIR detector ranges between $423 \mathrm{~nm}$ to $1000 \mathrm{~nm}$. Its SSI ranges from 4 to 10 $\mathrm{nm}$, generally increasing with increasing wavelength, and the bandwidth ranges from $5 \mathrm{~nm}$ to $12 \mathrm{~nm}$, also increasing with increasing wavelength. The SWIR detector covers a wavelength range from $900 \mathrm{~nm}$ to $2438 \mathrm{~nm}$. It has a SSI of 7 to $10 \mathrm{~nm}$, typically decreasing with increasing wavelength and a bandwidth ranging from $8 \mathrm{~nm}$ to $14 \mathrm{~nm}$, mainly decreasing with increasing wavelength. EnMAP SRFs were simulated using Gaussian functions, which had been generated specifically for each spectral band. The radiance was resampled along 
each column in the data to ensure accurate simulation of the spectral smile (Guanter et al., 2009). The result of the spectral simulation was data, resembling the spectral configuration of the EnMAP sensor.

\subsubsection{Radiometric Module}

The radiometric module is the final module in the forward simulation of the EnMAP data. It transforms the at-sensor radiance to photons, electrons and finally to DN (Segl et al., 2012). This module adds noise to the data and computes the calibration gain and offset coefficients (Guanter et al., 2009). It considers the quantum efficiency of the two detectors, optical transmittance, detector element size, spectral width and wavelength, the F-number of the optics, the readout noise for both detectors in low-gain and high-gain modes, shot noise, the dark current for each detector, and the Analog to Digital Converter (ADC) noise for both the high-gain and low-gain modes. The noise is based on parameters already measured for BOL (optical transmittance, quantum efficiency and readout noise) and parameters defined by the engineers. Variable non-linear responses for each detector element are also included in this module. The data output by the radiometric module simulated raw EnMAP data and was used as input for the backward simulation.

\subsubsection{Backward Simulation}

The next three modules are related to the backward simulation component of the EeteS simulation software. These modules reflect, but are not identical to, 
the processing steps that will be employed once the sensor is launched to generate the EnMAP data products. The process consists of four core steps, the Level-1 (L-1) processor, co-registration of the two detector images, atmospheric correction (L2atm) and orthorectification (L2geo) (Segl et al., 2012). EnMAP L-2 atmospherically and geometrically corrected data was simulated in this study.

\subsubsection{Level-1 Processor}

The L-1 processor is the first module to be applied in the backward simulation. Its purpose is to convert EnMAP DN values to TOA radiance and spatially co-register the two detector images. Estimated calibration parameters were used to convert the data from DN values to TOA radiances (Segl et al., 2012). Each detector element was calibrated using its own sensor calibration model. Four main processing steps are considered in the L-1 processor, the masking of saturated, bad and dead pixels, correction of non-linearity response, dark current subtraction and multiplication of gain coefficients (Segl et al., 2012).

The spatial co-registration of the two detector images was done by shifting the SWIR lines to the VNIR lines using the coordinates of the projected scan line centers (Segl et al., 2012). No resampling was performed in this step.

\subsubsection{Level-2 Processor}

The second module in the backward processing is the L-2 processor, which is comprised of the L2atm and L2geo. The purpose of the L2atm module is to convert simulated TOA EnMAP radiance values to surface reflectance values. 
The atmospheric correction was done using a modified version of the MODTRAN4 atmospheric RT code (Berk et al., 1998). These modifications were to generate faster executable files, with no changes made to the MODTRAN4 physical formulations (Guanter et al., 2007). All input parameters for the L2atm, relating to the scene characteristics, were kept the same as for the AISA atmospheric correction. Input parameters relating to, or influenced by, the sensor characteristics were changed accordingly. A scene-DEM was not used for the L2atm. Parameters, such as the viewing zenith angle, the relative azimuth angle, the CWV and the visibility, were calculated on a per-pixel basis. Linear interpolation was used to generate the zenith and azimuth parameters, while exponential interpolation was used to generate the CWV and visibility parameters for each pixel (Guanter et al., 2007).

For the data simulation neither spectral smile nor adjacency effects were added. The second module in the L-2 processor is the L2geo module, which performs the correction of all spatial aberrations within a parametric orthorectification process. The orthorectification was applied separately for each detector image. The individual pointing characteristics of each detector element and the position of the sensor are used in this process (Segl et al., 2012). For the orthorectification process, different resampling methods are available including, nearest neighbour, bilinear interpolation, and cubic convolution (Segl et al., 2012). Bilinear interpolation was chosen for this simulation because it was a 
compromise between the possible loss of spatial quality associated with nearest neighbour (Schläpfer et al., 2000) and the alteration of brightness values characteristic of cubic convolution (Richards, 2013). The result of the L-2 processor was an orthorectified EnMAP surface reflectance data cube. Table 3.6 outlines the performance characteristics of the future EnMAP satellite sensor.

Table 3.6: Performance characteristics of the future EnMAP sensor. Information was gathered from Kaufmann et al. (2012).

\begin{tabular}{|c|c|c|}
\hline Parameter & \multicolumn{2}{|c|}{ Performance } \\
\hline \multicolumn{3}{|c|}{ Satellite Characteristics } \\
\hline Imaging Principle & \multicolumn{2}{|c|}{$\begin{array}{l}\text { Pushbroom, two-prism imaging } \\
\text { spectrometers }\end{array}$} \\
\hline Spectral Characteristics & VNIR & SWIR \\
\hline Spectral Range (nm) & $420-1000$ & $900-2450$ \\
\hline Bands & 88 & 154 \\
\hline SSI & $6.5 / 10$ & 10 \\
\hline SNR & $>400: 1$ at $495 \mathrm{~nm}$ & $>170: 1$ at $2200 \mathrm{~nm}$ \\
\hline \multicolumn{3}{|c|}{ Spatial Characteristics } \\
\hline GSD (m) & \multicolumn{2}{|c|}{30} \\
\hline Swath $(\mathrm{km})$ & \multicolumn{2}{|c|}{30} \\
\hline \multicolumn{3}{|c|}{ Temporal Characteristics } \\
\hline Repeat Cycle (days) & \multicolumn{2}{|c|}{27} \\
\hline $\begin{array}{l}\text { Off Nadir Revisit Time } \\
\text { (days) }\end{array}$ & \multicolumn{2}{|c|}{$<4$} \\
\hline
\end{tabular}




\subsection{Image Processing}

A spectral soil classification was conducted on both the AISA and the simulated EnMAP data. To reduce complexity and computational times, both the AISA and the simulated EnMAP data, were subset to include only the SU field site. The SU subset and the SU field sampling sites can be seen in Figure 3.16. It is within the SU subset that the soil classification and validation was conducted.
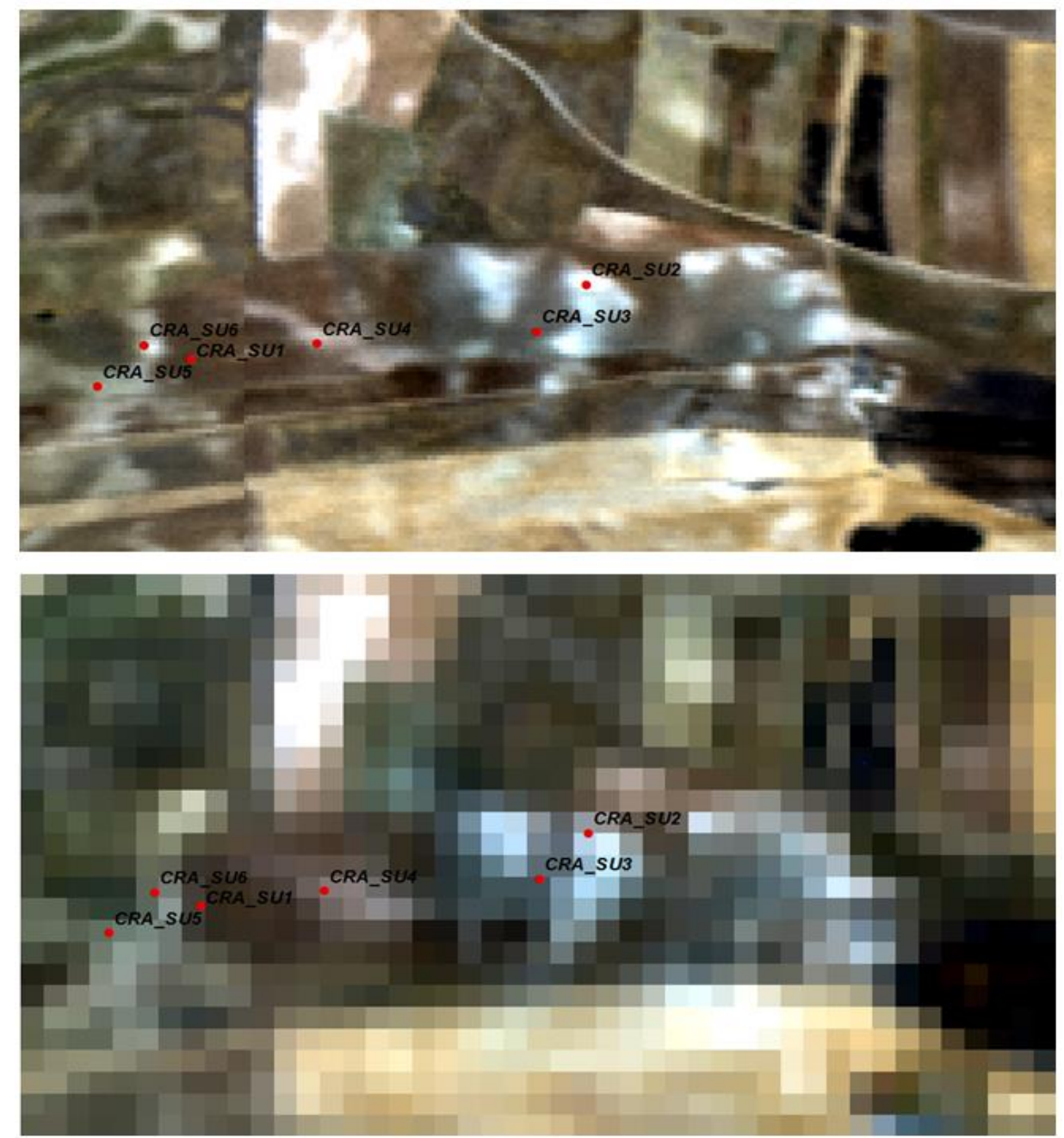

Figure 3.16: AISA SU subset (top) and EnMAP SU subset (bottom). Both images have the SU-field sampling sites highlighted with labeled red dots. 
The SU field site is a location where bare soil is known to be exposed at the surface. Within the areas of bare soil exposure, field data confirmed sites where stages of erosion, intermediate and accumulation are present. Also present in the image subset were areas of green vegetation and senesced vegetation.

A series of procedures were required to successfully classify the soils within the SU subset. In the first step, the vegetation was masked due to the potential influence on the soil analysis (Daughtry et al., 2004 and 2005). The vegetation was masked based on its fractional cover within each pixel, which was estimated using MESMA (Franke et al., 2009).

Once the vegetation was removed, the remaining soil dominated pixels were separated into erosion stages. The different stages used in this study were eroded, intermediate and accumulated. The intermediate class was defined by pixels, which have fraction values that fall outside the fraction range of the eroded and accumulated classes. Pixels in this class could also be referred to as areas of transition, because the class is largely defined by sloped positions that fall between the upper eroded area and the lower accumulation area.

The separation of the soil dominated pixels into stages was a two-step process, requiring first the quantification of representative soil characteristics present in each pixel and, secondly, the identification of class boundaries. The identification of class boundaries from the fraction image outputs was carried 
out using the topographic characteristics derived from the DEM (Irvin et al., 1995; Moore et al., 1993).

The unmixing of soil states was also conducted using MESMA. MESMA was chosen for the unmixing procedures for both the vegetation masking and the soils because of the high spatial variability of surface cover within the scene. By separating the image unmixing in to two modules, it created a hierarchical scenario, where the initial, less complicated unmixing procedure (vegetation and soils) provides a spatial constraint for the second, more complex unmixing procedure (soil erosion stages). This method was also applied in Franke et al. (2009) and Palacios-Orueta et al. (1999).

\subsubsection{Vegetation Mask}

Creating a vegetation mask is often the first step towards creating a soil classification map (Chabrillat et al., 2011; Hill and Schutt, 2000; Palacios-Orueta et al., 1999; Palacios-Orueta and Ustin, 1998). Spectral mixing of green and senesced vegetation with the underlying soil makes it difficult to identify or quantify the soils characteristics (Daughtry, 2001; Roberts et al., 1993). It has been observed that a vegetation cover exceeding $30 \%$ is an adequate threshold as to not have the effect of vegetation cover on any further soil analysis (Bartholomeus et al., 2007; Chabrillat et al., 2002; Tueller, 1987). Accordingly, any pixels exceeding this threshold were removed from further analysis. As mentioned above, the same procedure was applied to the images with the only difference 
being the spectra used for each endmember in the vegetation mask. This is because the endmember spectra were collected separately from both the AISA and the simulated EnMAP data sets.

Visual comparison between the AISA vegetation mask and the sample field sites was conducted to evaluate the accuracy of the vegetation mask. CAI and the Red-Edge Normalized Difference Vegetation Index (RENDVI) were also calculated for both the AISA and simulated EnMAP images and were used in a visual comparison against the respective vegetation masks (Nagler et al., 2003; Hurcom \& Harrison, 1998). The RENDVI index differs from NDVI only in the sense that is intended to be used with high-spectral resolution reflectance data, whereas NDVI was designed for broadband sensors.

\subsubsection{Endmember Selection and Unmixing}

The endmembers selected for the unmixing were green vegetation, dry vegetation, including burned vegetation, soils and shade. The spectra selected for each endmembers were chosen using the PPI (Boardman et al., 1995). A total of 49 and 38 pixels with the largest PPI values were selected for the AISA and simulated EnMAP images, respectively. The spectral characteristics of the selected PPI pixels as well as the spatial positioning of the pixels were examined to select the final spectra for each endmember. The unmixing was run with the default constraints for maximum fraction value (1.05), minimum fraction value ($0.05)$ and maximum shade fraction value (0.80). 
Through iteratively unmixing and selecting spectra from high RMSE areas, a total of 26 spectra for both the AISA and simulated EnMAP image were chosen for the final unmixing. For this purpose, two green vegetation endmember spectra, 12 dry and burned vegetation spectra, 12 soil spectra and the default photogrammetric shade were included for unmixing the AISA data, while four green vegetation spectra, 11 dry and burned vegetation spectra, 11 soil spectra and the default photogrammetric shade were utilized for the simulated ENMAP data. Figures 3.17 and 3.18 show the endmember spectra used for the unmixing of the AISA and simulated EnMAP data, respectively. 


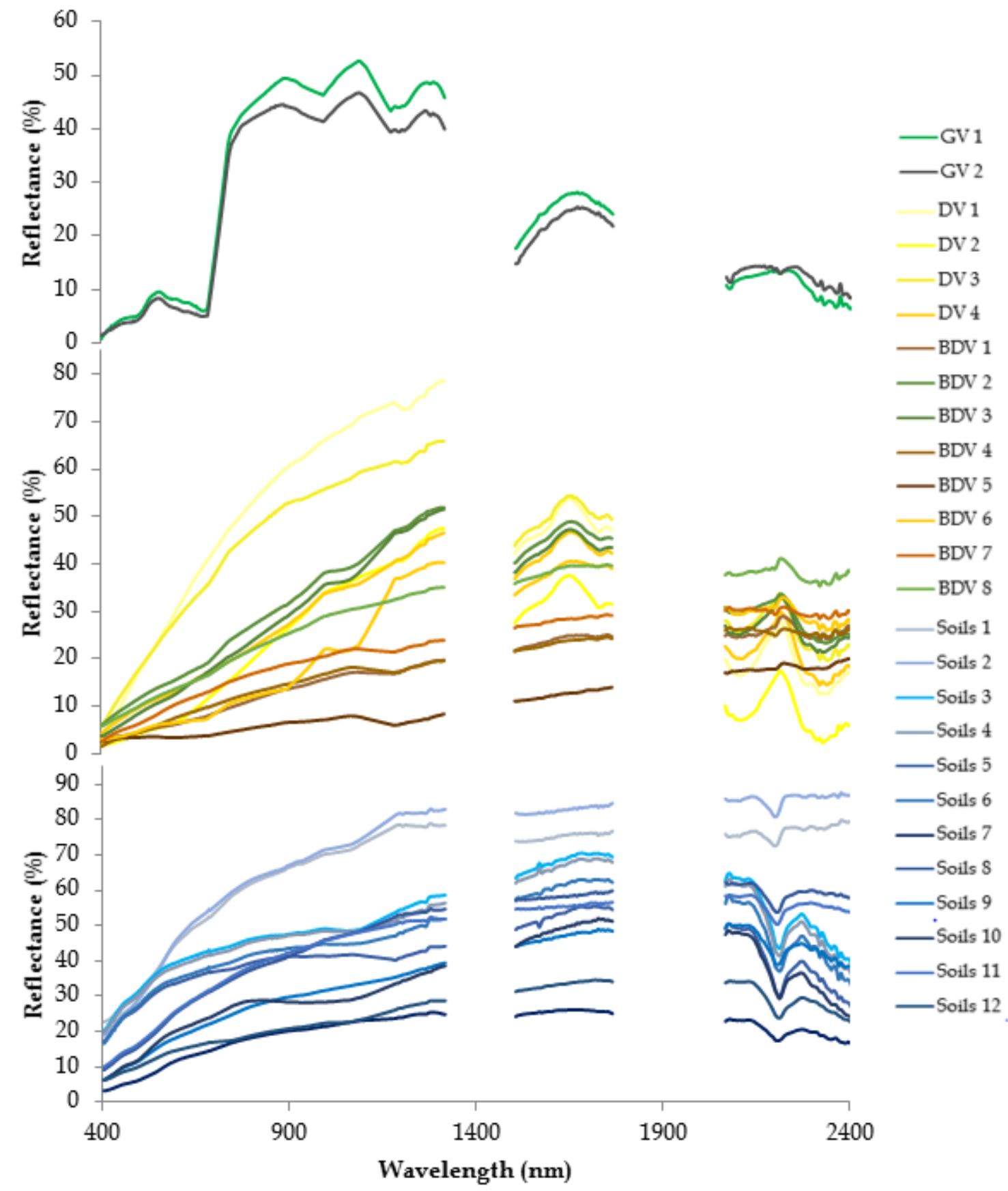

Figure 3.17: Endmember spectra used for unmixing the AISA data with the green vegetation endmember (top), dry vegetation endmembers (middle) and soil endmembers (bottom). 


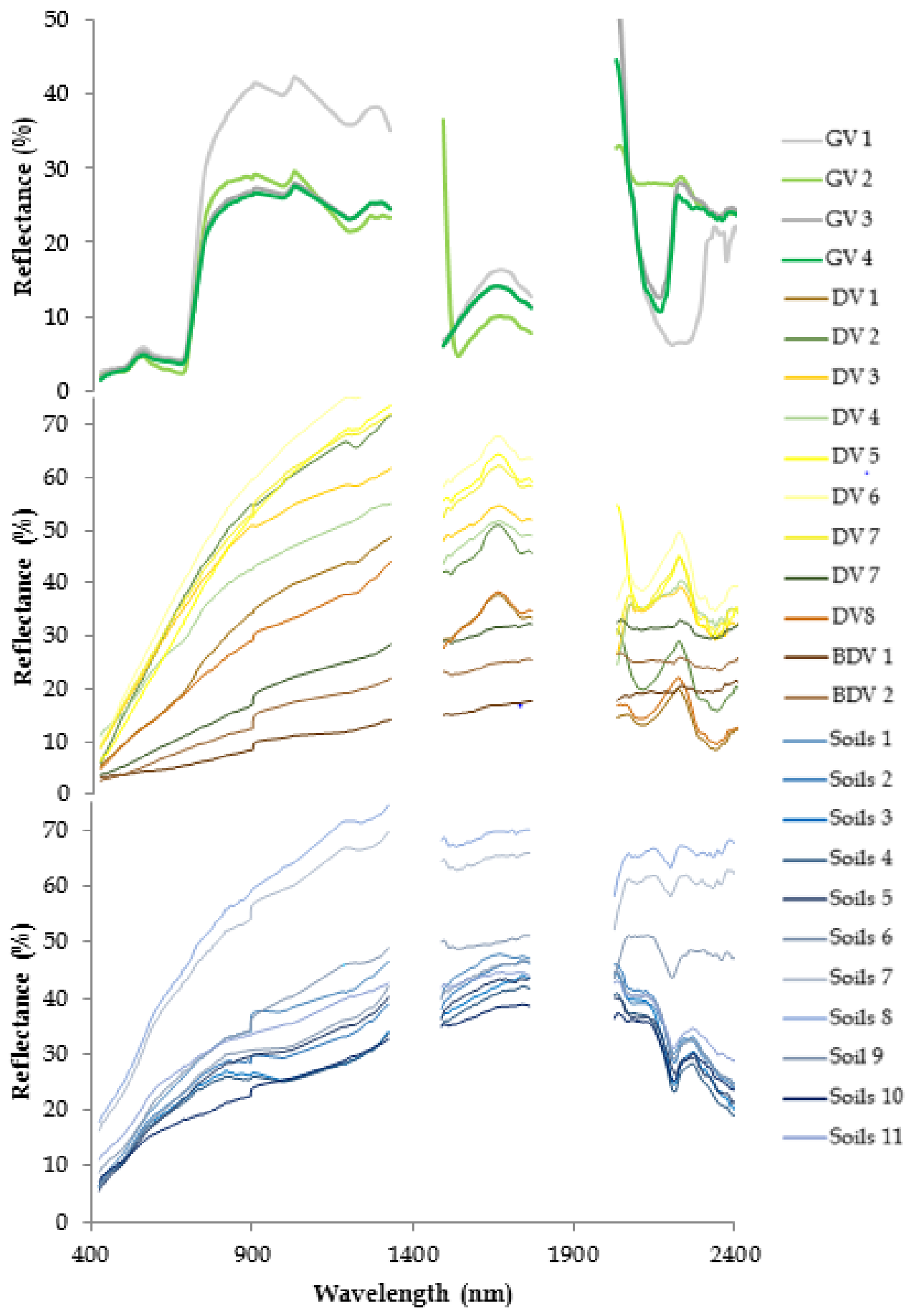

Figure 3.18: Endmember spectra used for unmixing of the EnMAP data with the green vegetation endmembers (top), dry vegetation endmembers (middle) and soil endmembers (bottom). A simulation effect can be seen in the SWIR region of the green vegetation spectra. 
A Red, Green and Blue (RGB) composite image for both the AISA and simulated EnMAP fraction images can be seen in Figure 3.19. It shows the distribution of green vegetation, soil and dry vegetation within the scene. The resulting fraction maps from each unmixing procedure were then shade normalized.
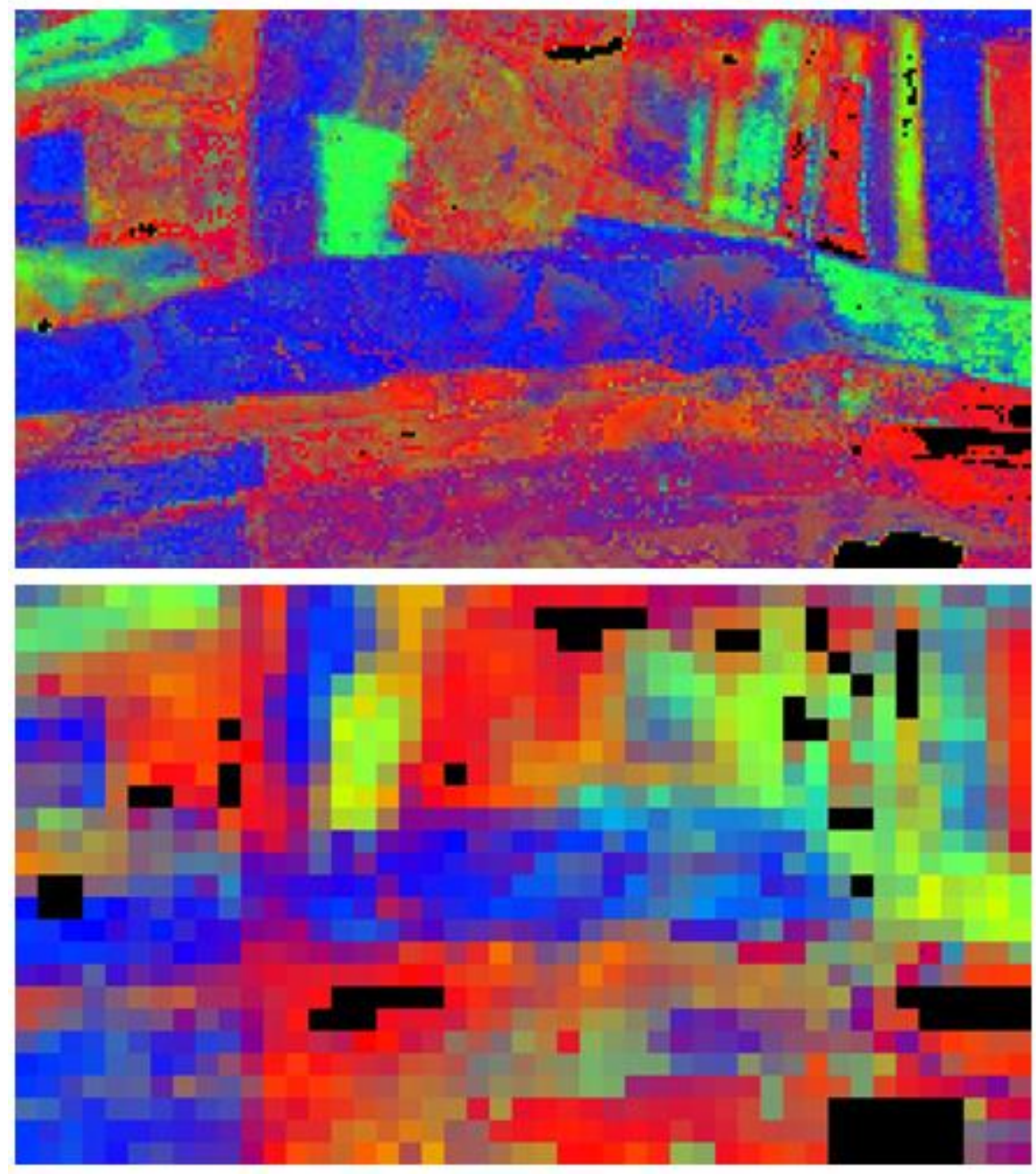

Figure 3.19: RGB composite of the endmember fractions for the AISA (top) and EnMAP (bottom). $R=$ dry vegetation, $G=$ green vegetation and $B=$ soil. 


\subsubsection{Mask Generation}

To determine the total combined vegetation fraction cover for each pixel, the shade-normalized green vegetation and dry/burned vegetation fraction maps were added. Following the summation a $0.3(30-\%)$ threshold was applied to mask pixels with a total vegetation cover greater than $30 \%$. Images of the masked AISA and simulated EnMAP data can be seen in Figure 3.20. Although a similar pattern can be seen in both of the vegetation masks, it is clear that a substantial amount of detail is missing from the simulated EnMAP vegetation mask compared to the AISA vegetation mask.
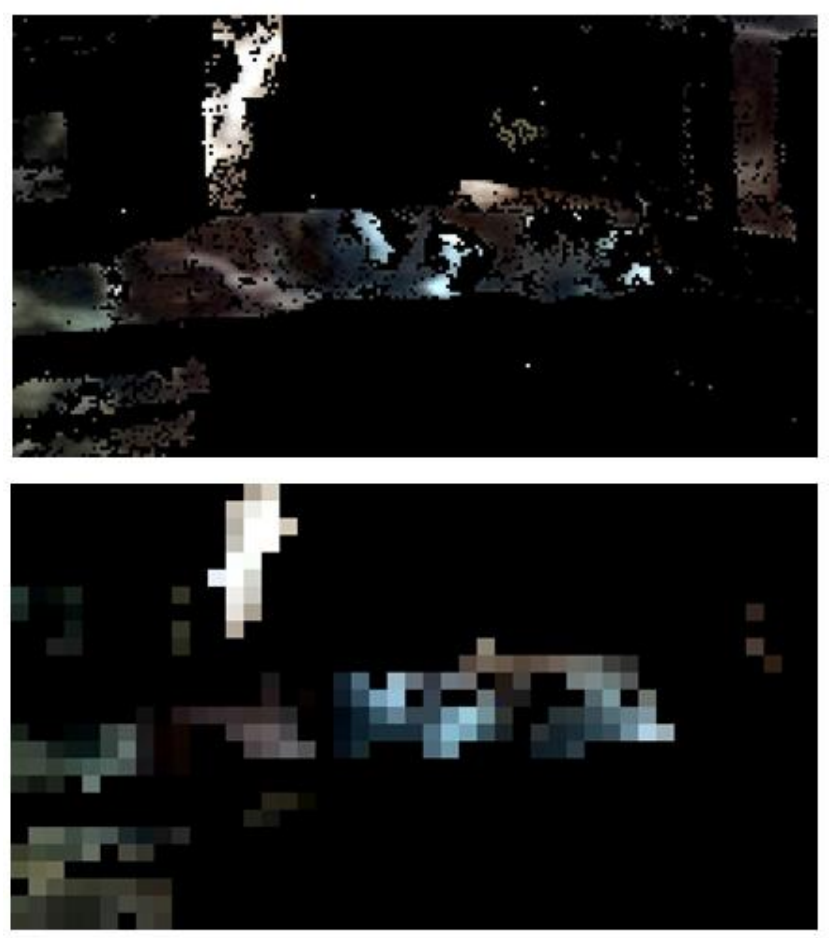

Figure 3.20: AISA (top) and EnMAP (bottom) images with the vegetation mask overlaid. Only visible in these images are the soil dominated pixels. 


\subsubsection{Soil Unmixing}

A three-endmember model in MESMA was applied to both the AISA and simulated EnMAP images. The goal of the soil unmixing was to quantify the contribution of both the eroded and the accumulated surface cover based on the spectral signature of each pixel in the scene. Assuming that the reflectance of each pixel in the image is a linear combination of the reflectance of each material present within the sensor's IFOV, the abundance of eroded material and accumulated material within each pixel can be estimated (van der Meer, 2002; Settle \& Drake, 1993). The erosion state of each pixel was then determined and classified based on the abundance of each of these materials within the pixel (Hill et al., 1994).

Validation of the AISA classification was carried out directly against the field data, while the validation of the simulated EnMAP classification was compared to the AISA classification using a confusion matrix. In this case, the AISA classification represented the reference image. For the confusion matrix to be generated, it required a rescaling of the AISA fraction images to $30 \mathrm{~m}$.

\subsubsection{Endmember Selection and Unmixing}

The endmembers used in the model were eroded, accumulated and shade. They were selected to represent the two extreme stages of soil erosion. Only the two classes instead of including a third, intermediate class, were chosen because no precise definition for the intermediate soil erosion class could be identified 
from the ground data. As a result, by employing only the two extreme soil erosion stages in the unmixing procedure, the soil condition at each pixel could then be defined as a function of the mixing ratio of the two endmembers.

Endmember spectra were collected based on the known field locations in the scene. Attempts were made to collect endmember spectra using other methods such as PPI and SMACC. Consequently, the endmembers needed to be selected using independent knowledge of the scene. For both scenes, the endmembers were selected based on the SU2 (eroded) and SU4 (accumulated) field sampling sites.

The pixels at these locations as well as the three surrounding pixels for each were collected and used to define the endmembers for the AISA image. This equals four spectra, representing the eroded endmember and four spectra representing the accumulated endmember. The spectra selected for the AISA unmixing are shown in Figure 3.21. 


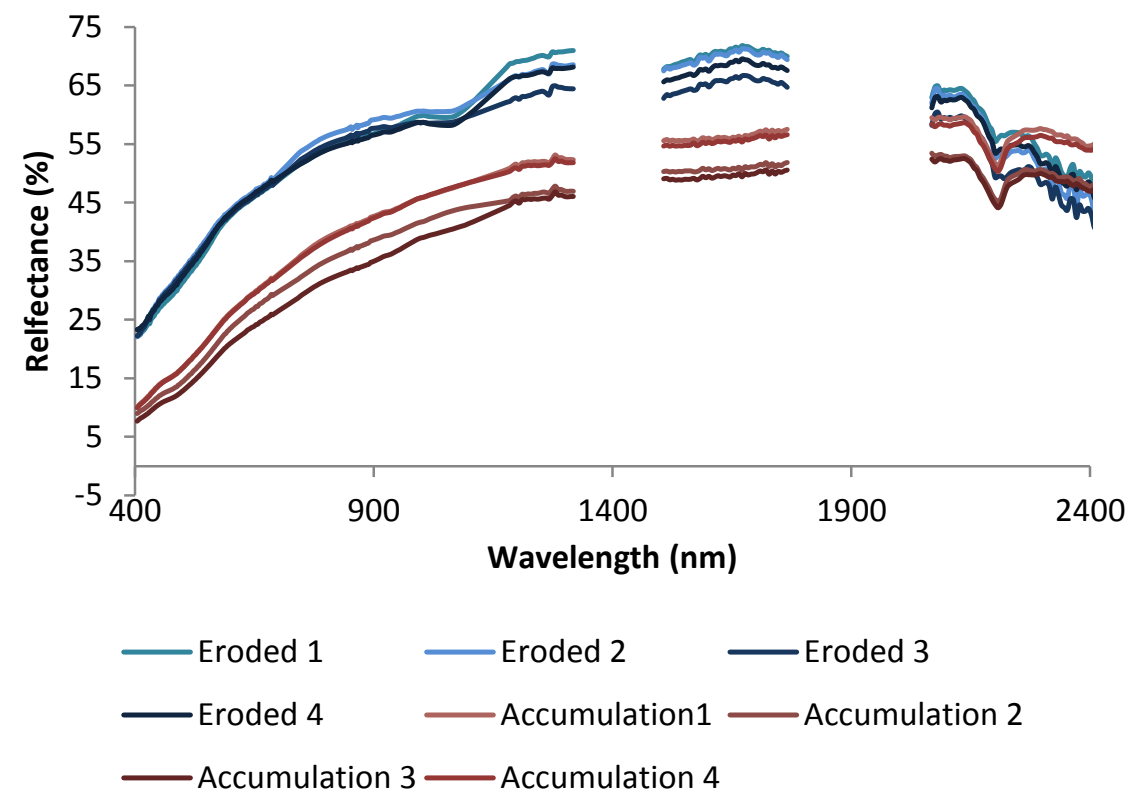

Figure 3.21: Spectra collected for both the eroded and accumulated endmembers used for unmixing the AISA image.

Only three spectra were collected for the EnMAP image unmixing, because there are far fewer pixels within the image. Accordingly, one spectrum representing the eroded endmember and two spectra representing the accumulated endmember were extracted from the imagery. Only the pixel at the location of the SU2-field site was used for the eroded endmember, because the surrounding pixels were spectrally very similar. There were two spectra used to represent the accumulated endmember in the unmixing, the SU4 location and the adjacent pixel. Two pixels were chosen for the accumulated endmember, because the two pixels varied in brightness. The spectra used for the EnMAP unmixing can be seen in Figure 3.22. 


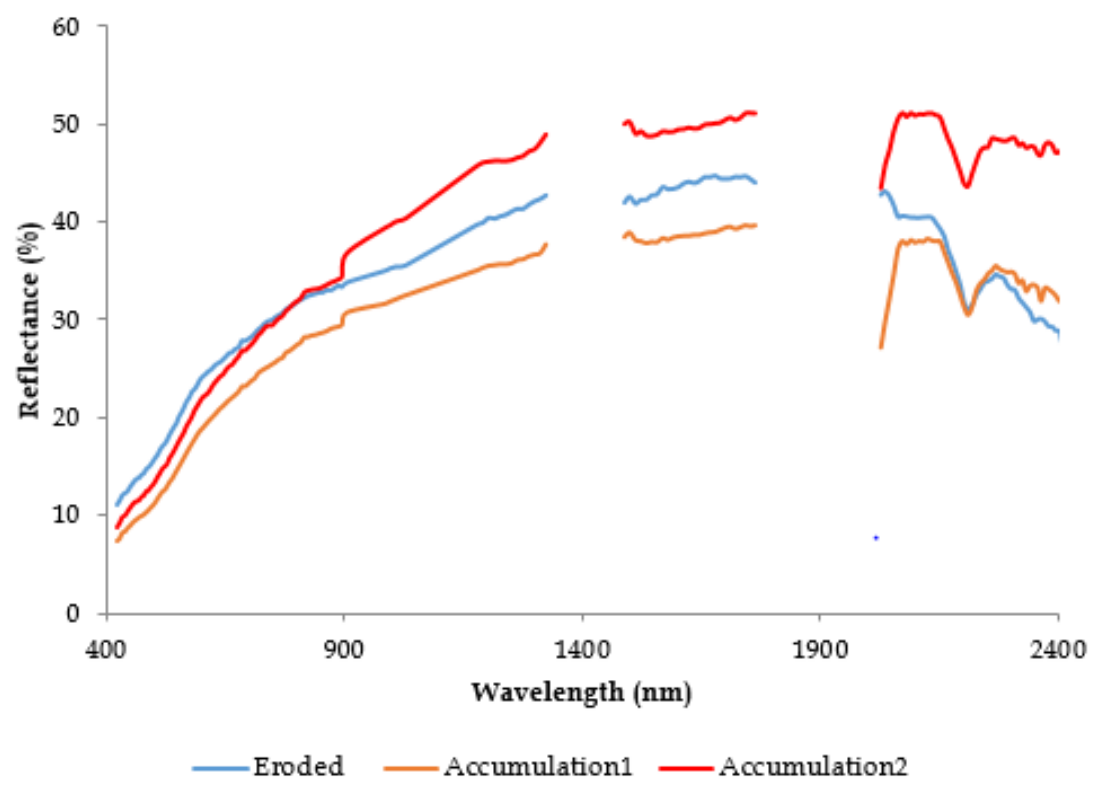

Figure 3.22: Spectra collected for both the eroded and accumulated endmembers used for unmixing the EnMAP image.

The scene was unmixed using MESMA with the default constraints for maximum fraction value (1.05), minimum fraction value (-0.05) and maximum shade fraction value (0.80). The fraction maps for the eroded and accumulated endmembers from both unmixings were then shade normalized. The fraction maps for the AISA and simulated EnMAP unmixing can be seen in Figure 3.23. 

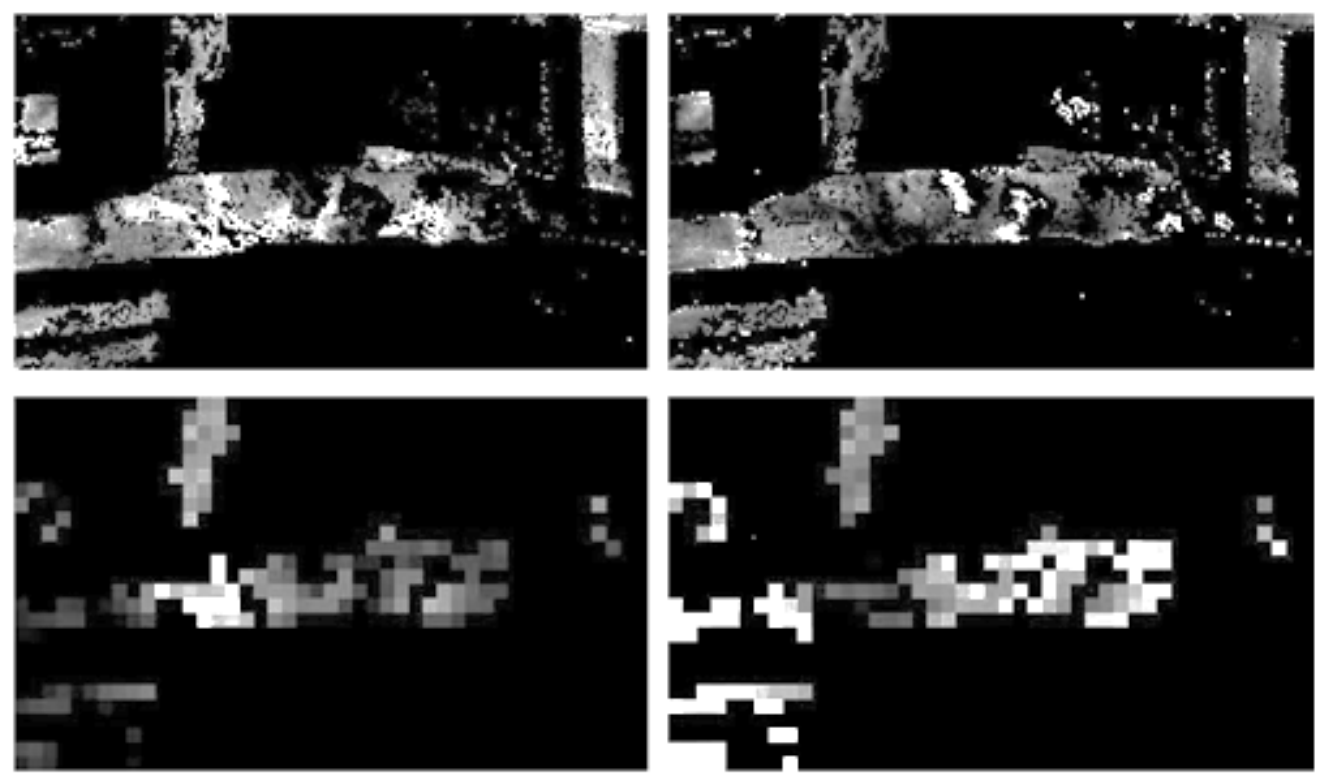

Fraction Value

0

Figure 3.23: AISA accumulated fraction map (top left), AISA eroded fraction map (top right), EnMAP accumulated fraction map (bottom left) and EnMAP eroded fraction map (bottom right). In all images, brighter areas represent higher fraction values.

\subsubsection{Terrain Analysis}

A DEM was generated using the LiDAR data collected during the campaign flight. The DEM was created using a 30-m by 30-m search radius and an X, Y grid line spacing of $30 \mathrm{~m}$. Inverse Distance Weighting (IDW) was chosen for the gridding of the data (Gotway et al., 1996; Weber and Englund, 1992). IDW is a method of interpolation where the assigned values are calculated using a weighted average of the values of the available points. IDW was chosen over 
other methods such as kriging and triangulation for two main reasons. Firstly, triangulation often causes artifacts or noise in the grid when used with LiDAR data, because of the inherent quantity of points in these types of data sets. Secondly, unlike kriging, IDW does not generate new data values, which maintain the overall integrity of the data. The resulting 30-m DEM removed unnecessary surface detail, while still providing useful topographic information. The DEM shown in Figure 3.24 provides a visual of the elevation differences present in the scene. The DEM was used to derive curvature, upslope contributing area, and slope. The values of each derivative gathered from the six field locations are listed in Table 3.7. These outputs, excluding the surface elevation, were then layered into a single image file and used to define the soil class boundaries. Images of the curvature, upslope contributing area and slope are shown in Figure 3.25. 

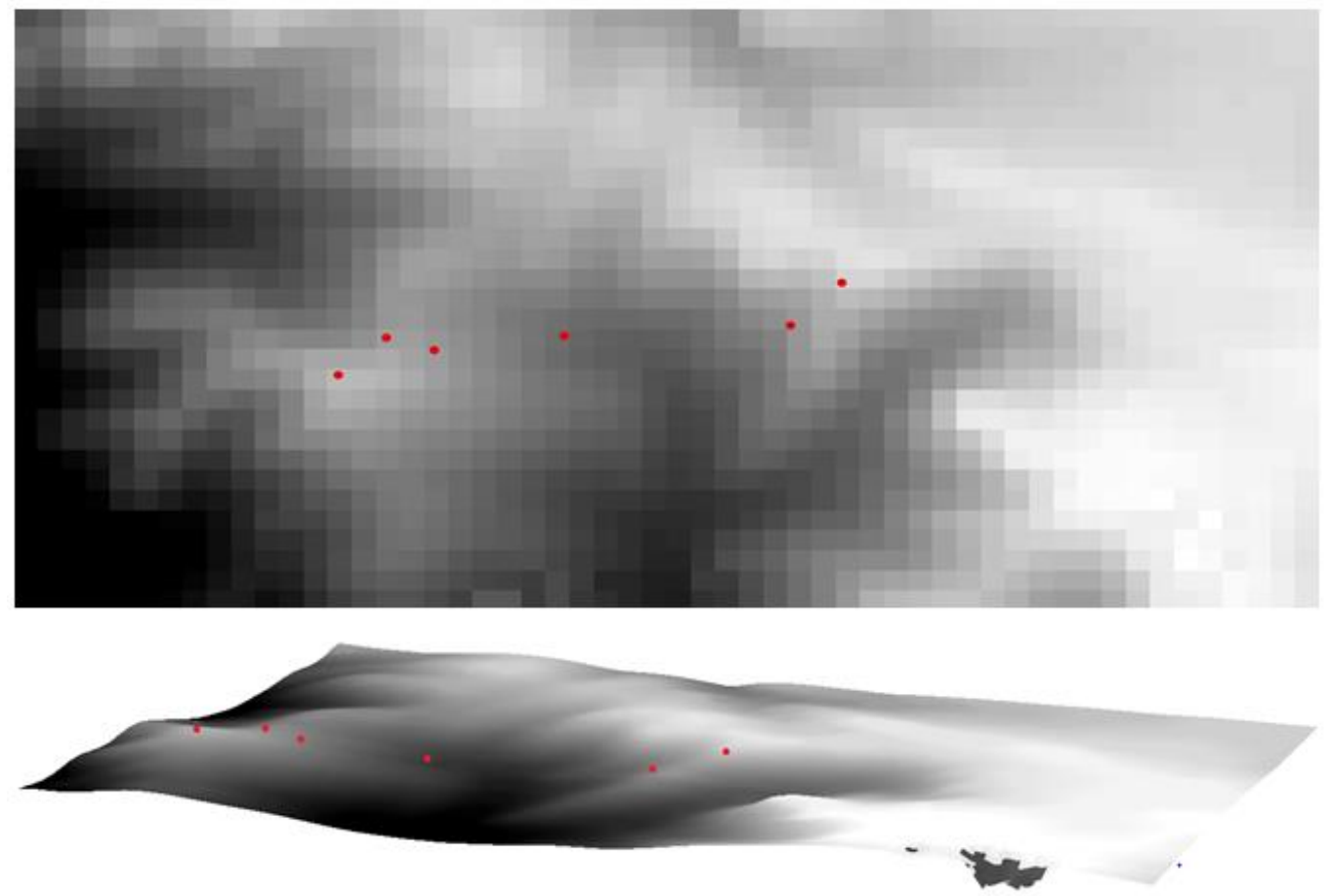

Figure 3.24: SU site DEM (top) and a 3D view of the SU site DEM (bottom). The SU field sites are highlighted with red dots.

Table 3.7 Elevation, curvature, upslope contributing area and slope values collected for each of the SU field sites. The definition of curvature is taken from Wilson \& Gallant (2000).

\begin{tabular}{|c|c|c|c|c|c|}
\hline & & $\begin{array}{c}\text { Elevation } \\
\mathbf{( m )}\end{array}$ & Curvature & $\begin{array}{c}\text { Upslope } \\
\text { Contributing } \\
\text { Area (m) }\end{array}$ & $\begin{array}{c}\text { Slope } \\
\text { (deg) }\end{array}$ \\
\hline SU2 & $\begin{array}{c}\text { Most } \\
\text { Advanced } \\
\text { Erosion }\end{array}$ & 624.29 & Concave & 60 & 4.316 \\
\hline SU6 & $\begin{array}{c}\text { Advanced } \\
\text { Erosion }\end{array}$ & 619 & Concave & 90 & 2.52 \\
\hline SU5 & Erosion & 622 & Convex & 30 & 1.15 \\
\hline SU1 & Intermediate & 618.45 & Convex & 30 & 2.27 \\
\hline SU3 & Intermediate & 617.14 & Concave & 90 & 3.47 \\
\hline SU4 & Accumulation & 613.02 & Concave & 180 & 1.51 \\
\hline
\end{tabular}



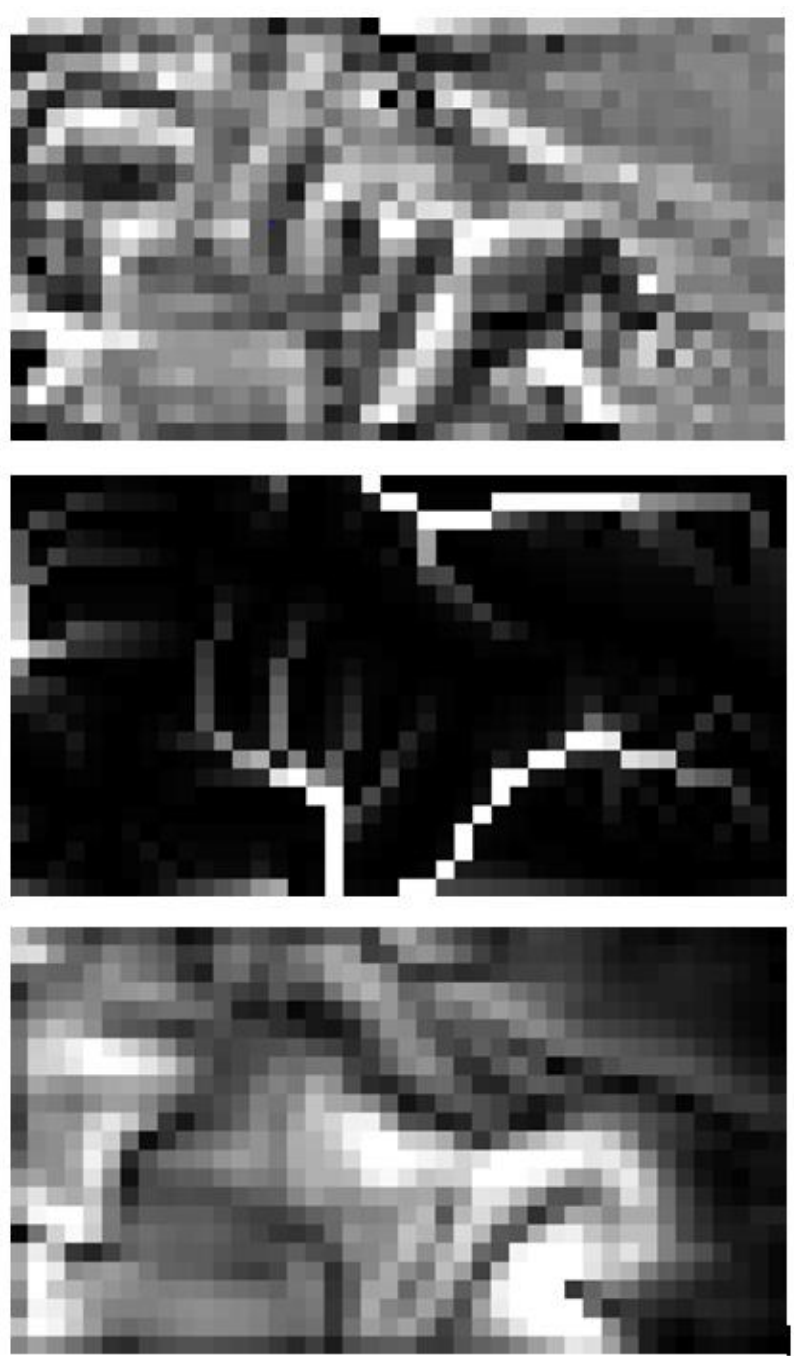

Figure 3.25: Images of curvature (top), upslope contributing area (middle) and slope (bottom).

\subsubsection{Spectral Soil Erosion Class Generation}

The classes chosen for the study site were eroded, intermediate and accumulated. The output fraction maps for the eroded and accumulated endmembers provided information about the proportion of each endmember within the pixel. The class boundaries were defined based on maximum and 
minimum allowable fraction values of the eroded and accumulated endmembers. The maximum and minimum fraction values were determined by classifying the SU site based on the terrain derivatives, using the respective range of fraction values within each class to define the spectral class boundaries. The resampled 30-m AISA data were used to generate the class boundaries for the AISA validation data.

The classification of the terrain derivatives was carried out using the Spectral Angle Mapper (SAM; Boardman \& Kruse, 1994). Because SAM is a supervised classification method, it was important to first identify the topographic characteristics associated with each soil state so that valid training data can be collected for the classification. This was done by creating a transect between the SU2 and SU5 field locations. Because all the field locations generally fall in a straight line between those two sites, the transect is able to provide an indication of the characteristics of the identified soil states. Figure 3.26 shows the transect that was taken across the scene. 

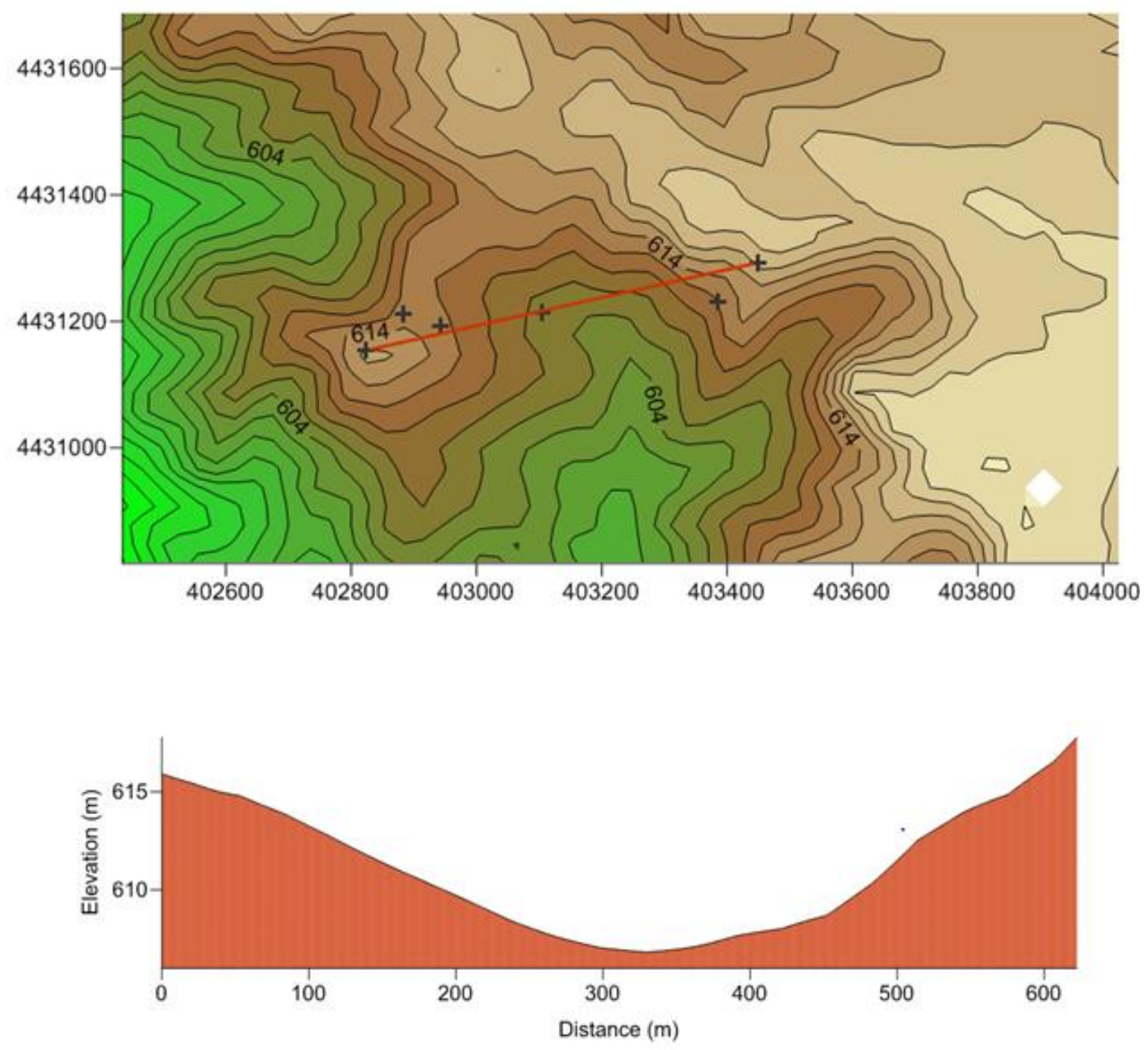

Figure 3.26: Contour map of the SU site (top) with the SU field locations indicated with black crosses and the transect line shown in red with elevation profile of the transect (bottom). In the elevation profile you would expect to see erosion occurring in the regions of higher elevation and the areas of lower elevation to be predominantly areas of accumulation. 
Training sets for the SAM terrain classification were selected for each soil erosion state. The training sets were selected based on their relative location to the field sampling locations. These training sets were used to classify the terrain into the three classes of eroded, intermediate and accumulated. The terrain classification was then overlaid on both the AISA and simulated EnMAP fraction images. The fraction values contained within each of the terrain classes were then recorded for both images. The maximum and minimum fraction values, means and standard deviations were calculated. These values are summarized in Table 3.8 .

Table 3.8: Maximum and minimum fraction values, means and Standard Deviation (Stdev) values collected for each erosion state from each fraction image.

\begin{tabular}{|c|c|c|c|c|c|c|c|}
\hline \multirow[t]{2}{*}{ Erosion State } & \multirow[t]{2}{*}{ Class } & \multicolumn{3}{|c|}{$\begin{array}{l}\text { AISA Accumulation } \\
\text { Fraction Image }\end{array}$} & \multicolumn{3}{|c|}{$\begin{array}{l}\text { AISA Erosion Fraction } \\
\text { Image }\end{array}$} \\
\hline & & Range & Mean & Stdev & Range & Mean & Stdev \\
\hline Eroded & 1 & $0.29-0.86$ & & 0.11 & $0.13-0.79$ & 0.36 & 0.13 \\
\hline Inte & 2 & $0.32-0$ & & & & & \\
\hline \multirow{3}{*}{ Accumulation } & 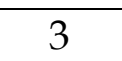 & $0.54-1.02$ & 0.82 & 0.14 & $-0.05-0.45$ & 0.16 & 0.14 \\
\hline & Class & \multicolumn{3}{|c|}{$\begin{array}{l}\text { EnMAP Accumulation } \\
\text { Fraction Image }\end{array}$} & \multicolumn{3}{|c|}{$\begin{array}{l}\text { EnMAP Erosion Fraction } \\
\text { Image }\end{array}$} \\
\hline & & Range & Mean & Stdev & Range & Mean & Stdev \\
\hline Eroaea & 1 & $-0.04-0.71$ & 0.22 & 0.22 & $0.28-1.04$ & 0.77 & 0.22 \\
\hline Intermedia & 2 & $-0.06-0.96$ & 0.32 & 0.29 & $0.37-1.06$ & 0.67 & 0.28 \\
\hline Accumulation & 3 & $0.69-1.00$ & 0.53 & 0.26 & $0.0-0.93$ & 0.46 & 0.26 \\
\hline
\end{tabular}


The values indicate that there is a substantial amount of overlap in fraction value ranges between the classes. The mean values, although demonstrating an appropriate trend, are still very closely clumped in both the AISA and the simulated EnMAP fraction images. Because there is such a high level of overlap between the ranges, the means and standard deviations were used to select appropriate fraction ranges for each class.

The class ranges were identified by defining one \pm standard deviation from each of the class means then further refining the classes based on the remaining overlap. Any overlap between classes was defined as the intermediate class because no distinct class could be distinguished. The fraction values that fell only into the eroded or the accumulated classes, with no overlap, were classed as eroded and accumulated, respectively. The final fraction ranges for each class are listed in Table 3.9. A confusion matrix was calculated between the AISA and the simulated EnMAP classifications to evaluate the accuracy of the results.

Table 3.9: Maximum and minimum fraction values used to define the erosion classes.

\begin{tabular}{|c|c|c|c|c|c|c|c|c|c|}
\hline & Class & $\begin{array}{c}\text { AISA } \\
\text { Accumulation } \\
\text { Fraction } \\
\text { Image }\end{array}$ & \multicolumn{2}{c|}{$\begin{array}{c}\text { AISA } \\
\text { Erosion } \\
\text { Fraction } \\
\text { Image }\end{array}$} & $\begin{array}{c}\text { EnMAP } \\
\text { Accumulation } \\
\text { Fraction } \\
\text { Image }\end{array}$ & $\begin{array}{c}\text { EnMAP } \\
\text { Erosion } \\
\text { Fraction } \\
\text { Image }\end{array}$ \\
\hline Eroded & 1 & 0 & 0.539 & 0.461 & 1 & 0 & 0.038 & 0.962 & 1 \\
\hline Medium & 2 & 0.540 & 0.868 & 0.132 & 0.46 & 0.039 & 0.615 & 0.385 & 0.961 \\
\hline Accumulation & 3 & 0.869 & 1 & 0 & 0.131 & 0.616 & 1 & 0 & 0.384 \\
\hline
\end{tabular}




\subsection{Summary}

AISA Eagle and Hawk hyperspectral and Leica ALS50 (II) airborne laser scanner airborne data were collected for the Camarena study site. The methods used for this analysis can be separated into three major sections. The first of these sections is the pre-processing of the AISA Eagle and Hawk hyperspectral data. The pre-processing consisted of radiometrically, geometrically and atmospherically correcting the data. Also included in the pre-processing was the cross-track illumination correction, mosaicking, MNF and ELC.

The second distinct section within the methods was the simulation of the EnMAP data. The EnMAP data simulation was carried out using EeteS. The preprocessed combined AISA Eagle and Hawk reflectance data cube was used as the input for the EeteS simulation software. The simulation process was comprised of two separate components, the forward and backward simulation. The final result of EeteS was a simulated Level-2 (L-2), atmospherically and geometrically corrected EnMAP reflectance image cube.

The final section of the methods consisted of the processing and analysis of the combined AISA Eagle and Hawk data and simulated EnMAP data, resulting in the final soil erosion state map. This consisted of the masking of dry and green vegetation, estimation of soil fractions and classification of the image scene into soil erosion states. 


\section{Results}

The results presented in this section assist in accomplishing the main goal of this research, which is to evaluate the capability of the future EnMAP satellite sensor to detect and map soil erosion in semi-arid Mediterranean agricultural regions. The process consisted of segregating soil dominated pixels (defined by a soil fractions $>70 \%$ ) and spectrally unmixing the resulting pixels to determine the eroded and accumulated fractions. Terrain analysis using a DEM determined from the collected LiDAR data assisted in generating the class boundaries for the soil erosion state classes. The results cover the three main segments discussed in the methods; the pre-processing of the AISA data, the EnMAP simulation and soil mapping, analysis and validation of the soil erosion state map.

\subsection{Image Pre-processing}

Many pre-processing steps required to generate the final combined AISA Eagle and Hawk reflectance data cube (Figure 3.6). The results present the evaluation of the output from the pre-processing components as well as any accuracy evaluation conducted on the output. The results for the pre-processing are presented in the order they were conducted.

\subsubsection{Radiometric Correction - Destriping}

The destriping procedure removed all of the striping features from the AISA image data (Figure 4.1). The validation for the destriping was conducted using a variety of methods. One method included the comparison of horizontal 
profiles that crossed one or more line features in the pre- and post-destriped images. The second method compared the spectral profile of a corrected stripe with the spectral profile of a neighbouring pixel within a homogeneous area to evaluate how closely the destriped spectra resemble the original image spectra. Lastly, the images were visually examined for any artifacts remaining from the destriping procedure. Validation was concentrated mainly in areas with ground reference data and in bands considered to be of interest, such as the 2300-nm region, where the carbonate feature is present.
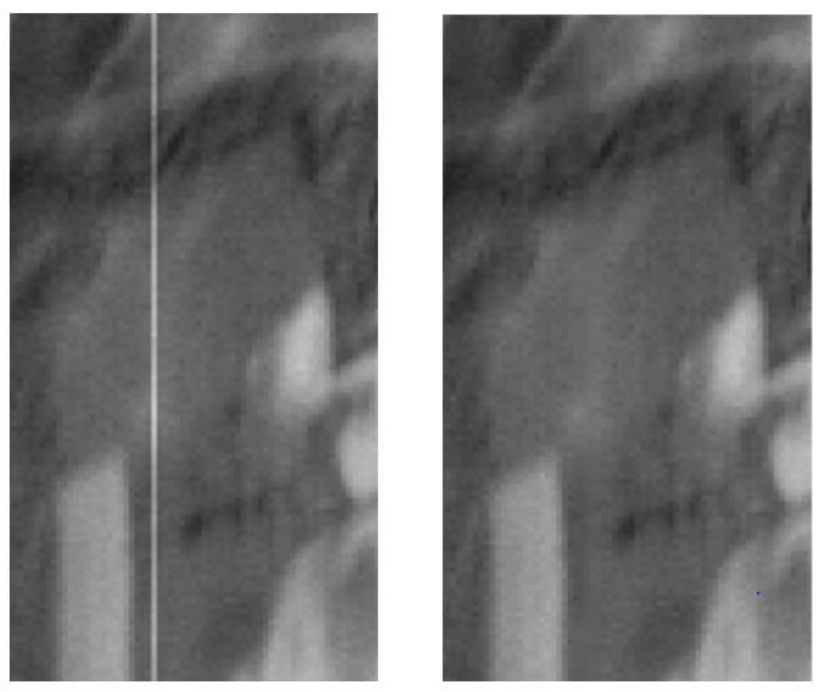

Figure 4.1: Example from before (left) and after (right) the destriping procedure.

Both the horizontal profiles and the spectral profiles confirmed that the destriping procedure adequately compensated for the stripes present in the original AISA Hawk flight lines. Figure 4.2 gives an example of a pre and post- 
destriping horizontal profile. The horizontal profile is for a single row in the 22004 flight line and extends the entire width of the flight line. Visual examination of the individual flight lines confirmed that there were no stripes remaining; however, some artifacts were found. This was done by looking at the bands that were previously recorded as having stripes and noting any missed stripes or artifacts.

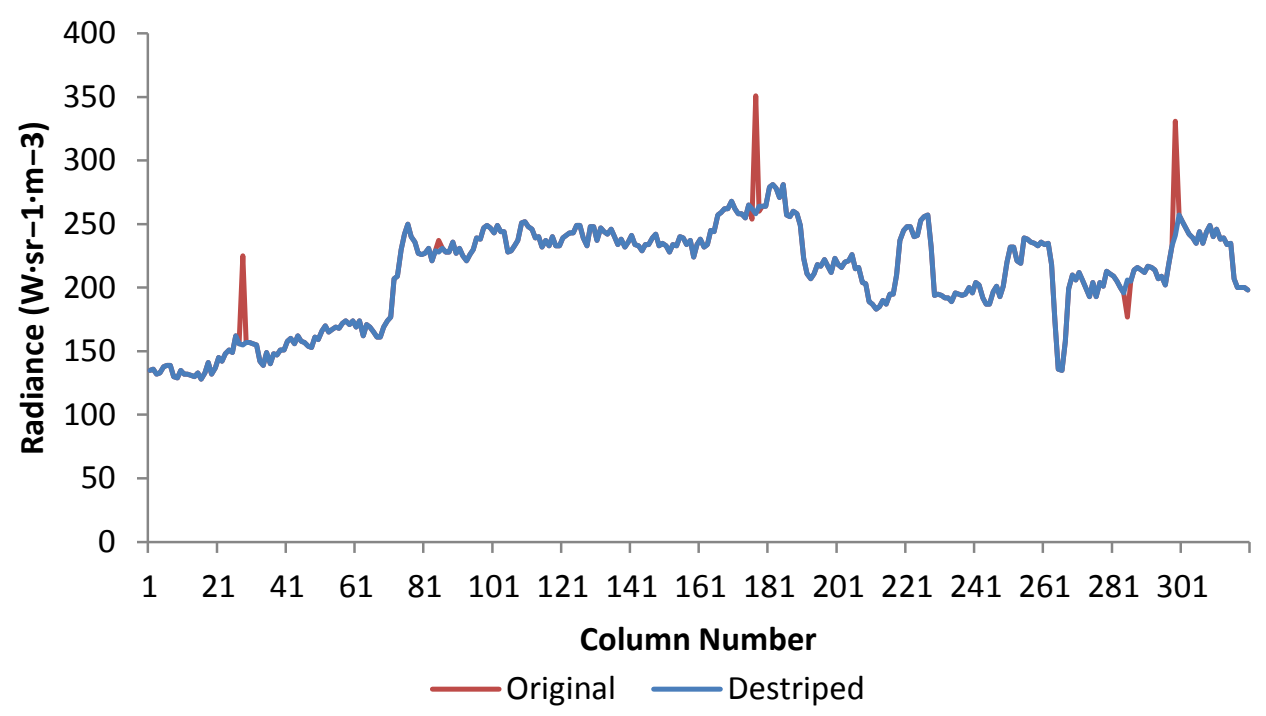

Figure 4.2: Comparison of a horizontal profile from flight line 22005 at $1443.8 \mathrm{~nm}$ before and after destriping. The horizontal profile extends the width of the flight line. The red spikes are the result of striping. Their locations show the columns where striping occurs. 
Visual inspection of spatially and temporally invariant features, such as roads and field boundaries, in each of the bands showed that there were a few instances of artifacts as a result of the destriping. Comparison between the striped image and the destriped image showed one instance of a false positive where destriping occurred in a location without stripes. From the visual examination conducted only two instances of blurring artifacts and one false positive were identified. An example of the blurring artifact can be seen in Figure 4.3.

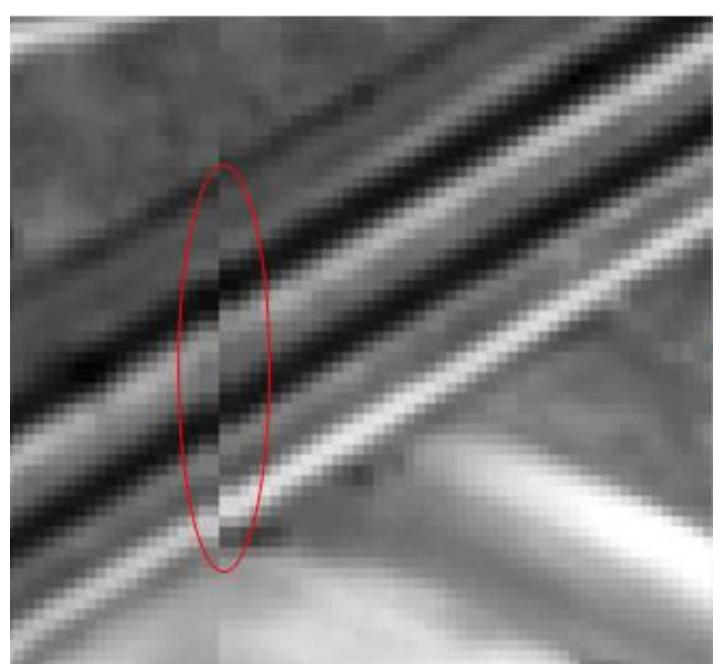

Figure 4.3: The artifact in flight line 22009, column 35 at $2232.4 \mathrm{~nm}$. The artifact is circled in red. 


\subsubsection{Geometric Correction}

Extensive geometric correction is required for images collected from airborne platforms before any further processing or analysis can be conducted. This is due to the complex geometric errors introduced by the dynamic movements experienced when collecting remotely sensed data using an airborne platform. In this research, the data was orthorectified using an in-house developed software. The results of the geometric correction were evaluated both visually and based on the RMSE. A preliminary mosaic of the combined AISA Eagle and AISA Hawk flight lines showed that the geometry of each flight line coincided accurately with one another with discrepancies of one or two pixels occurring at sporadic locations though the mosaic seam lines.

RMSE values for airborne images with GSDs between $1 \mathrm{~m}-10 \mathrm{~m}$ are ideally in the range of one pixel (Palubinskas et al., 2003). However, acceptable RMSE values range between 1 - 3 pixels (Schlapfer \& Richter, 2002). The RMS error for the geometric correction was calculated for both the individual flight lines and for the final mosaicked scene. The Spanish government online map, Sigpac (sigpac.mapa.es), was used for the geometric RMS error calculation. The GCPs collected from the Sigpac online map were gathered from digital orthophotos with a pixel sizes between $0.25 \mathrm{~m}$ to $0.50 \mathrm{~m}$. However, the geometric accuracy of the map is unknown. 
A total of 26 GCPs were collected for the RMSE evaluation of the individual flight lines and 72 GCPs were acquired for the RMSE calculation of the mosaicked scene. The average RMS error calculated for the individual flight lines was $11.33 \mathrm{~m}$, while the error for individual GCPs ranged from $2.00 \mathrm{~m}$ to $17.49 \mathrm{~m}$. The GCPs with the largest residuals were concentrated along the edges of the flight lines, decreasing towards nadir (Figure 4.4).

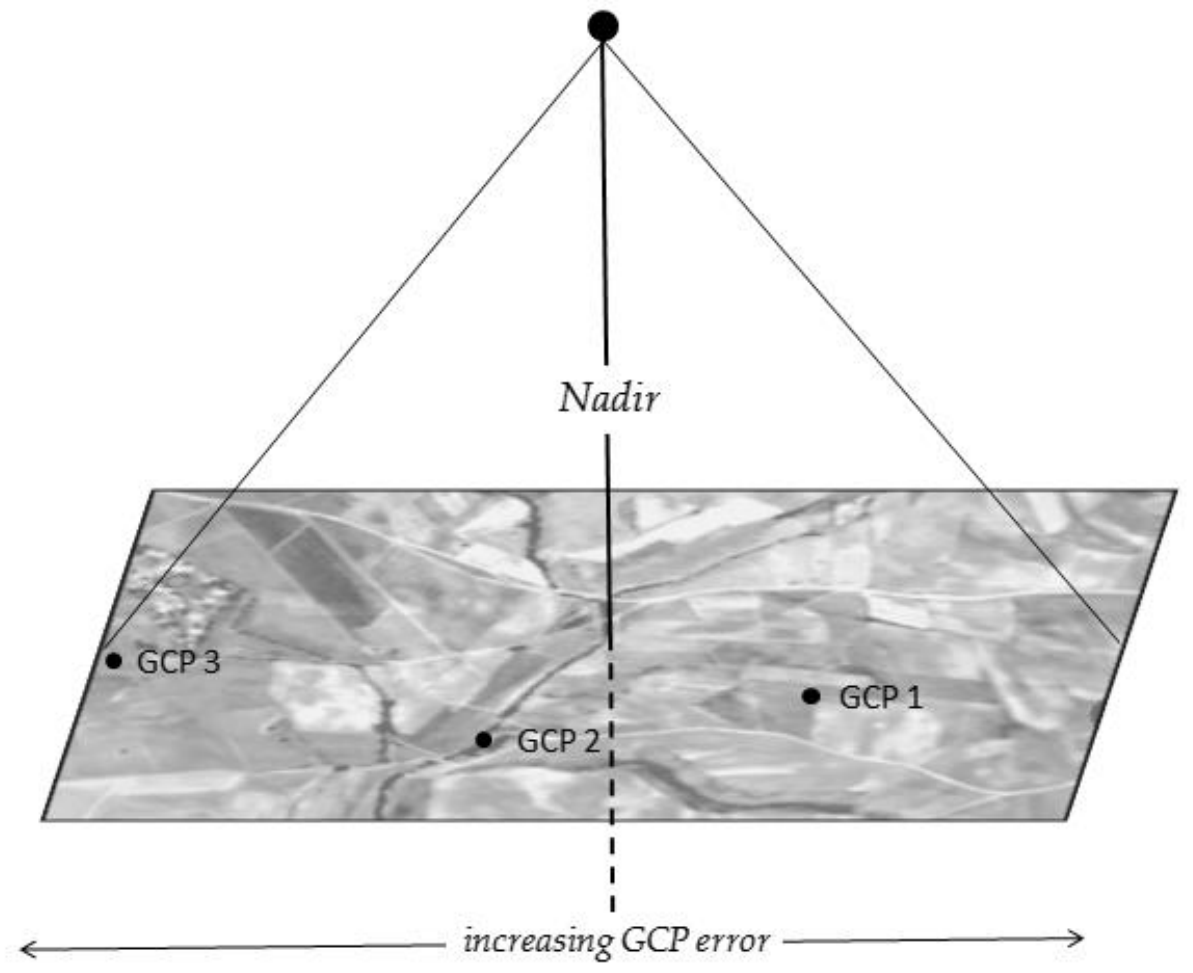

Figure 4.4: Individual GCP error increased towards the outer edges of the flight lines. In this example, GCP 3 will have a substantially larger residual than GCP 1 and GCP 2. 
The average RMS error for the final mosaicked scene was $5.96 \mathrm{~m}$, while the residuals for individual GCPs from the mosaicked scene ranged from $0 \mathrm{~m}$ to $12.04 \mathrm{~m}$. The drastic decrease in average RMS error between the individual flight lines and the final mosaic is a result of the spatial sub-setting of the flight lines prior to mosaicking. The removal of the edges, in turn, removed the more severe spatial offsets in the images.

\subsubsection{Data Fusion}

The data fusion was conducted for the purpose of combining the AISA Eagle and the AISA Hawk data into a single image file. The result of the data fusion was an image containing the both the AISA Eagle and Hawk data, covering the spectral range between $400 \mathrm{~nm}$ and $2400 \mathrm{~nm}$.

\subsubsection{Jump Correction}

The jump correction was conducted to account for a spectral offset introduced in the data fusion process. The results of the jump correction was the removal of the spectral offset, which can be seen in Figure 4.5 through the comparison of jump corrected and pre-jump corrected radiance spectra from a known field locations. 


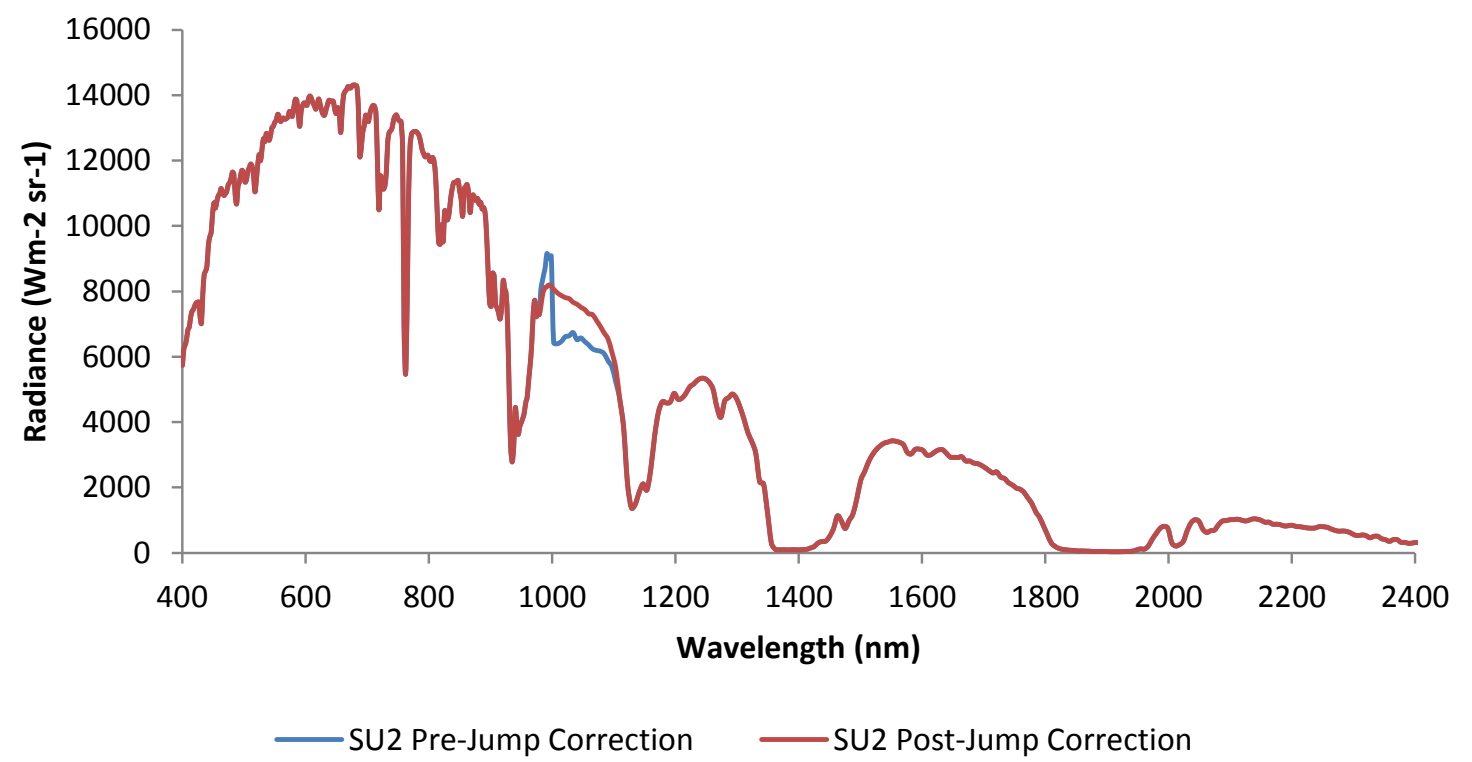

Figure 4.5: A comparison of the pre-and post- jump corrected spectra for the SU2 field location.

Two potential causes of the jump were investigated during the correction procedure. The first being the difference in pixel sizes $(2 \mathrm{~m}$ and $6 \mathrm{~m})$ and FOV angles (37.7 degrees and 24.0 degrees) between the AISA Eagle and AISA Hawk sensors. This would indicate an error in the co-registration of the images, thus, fusing the VNIR spectrum of one pixel to the SWIR spectrum of an adjacent pixel. The second cause that was investigated was a potential miscalibration between the VNIR and SWIR detectors in the $978 \mathrm{~nm}$ to $1096 \mathrm{~nm}$ region. Neither of the potential causes were successfully confirmed as the cause of the jump within the $900 \mathrm{~nm}$ to $1100 \mathrm{~nm}$ region. The final choice to replace the data between $978 \mathrm{~nm}$ and $1096 \mathrm{~nm}$ with the mixed spectrum was made as a result of the failure 
to recalibrate the data and by evaluation of the quality assessment provided by the ARSF.

\subsubsection{Atmospheric Correction}

The goal of an atmospheric correction is to remove all atmospheric scattering and absorption influences on a remotely sensed image and allow for the calculation of surface reflectance. ATCOR's rough terrain model was chosen because of topographically induced reflectance anisotropy. This concern has been made evident by Feingersh et al. (2007), indicated that slopes up to 25 degrees can change the results of a spectral interpretation of remotely sensed data considerably. A rural atmosphere with a water vapour content of $1.05 \mathrm{~cm}$, a flying altitude of $5000 \mathrm{~m}$ and a visibility of $80 \mathrm{~km}$ was applied to the scene. The rural atmosphere was chosen based on the largely agricultural land use within the scene. The $820 \mathrm{~nm}$ water absorption feature was chosen to estimate the water vapour content in the scene.

The $80-\mathrm{km}$ visibility used in the processing of the combined AISA Eagle and AISA Hawk data was estimated by ATCOR . The reason ATCOR would overestimate the visibility is the result of a specification in ATCOR that requires that no reflectance values be negative. Reflectance values at $660 \mathrm{~nm}$ in the red and $850 \mathrm{~nm}$ in the NIR are measured to determing if there are negative values in the scene. The visibility is increased until the reflectance values in these bands 
are preferably $>1 \%$. Bands in the $760 \mathrm{~nm} O_{2}$ region and the $725 / 825 \mathrm{~nm}$, 940/1130 $\mathrm{nm}$ and 1400/1900 $\mathrm{nm}$ water vapour regions were interpolated.

The solar azimuth and zenith angles were a variable and needed to be taken into consideration in the atmospheric correction since the flight duration was approximately 60 minutes. The solar azimuth angles ranged between approximately 239 degrees to 253 degrees (varied 14 degrees during acquisition) and the solar zenith angles ranged between approximately 37 degrees and 47 degrees (varied 10 degrees during acquisition). The main image acquisition was along the solar principal plane.

The total change in illumination was calculated for the scene using hillshades generated from the 2-m DEM created from the LiDAR data. The hillshades are a shaded relief map of the scene based on the local illumination angle and local shadowing. The first hillshade was generated with the solar azimuth and zenith angles from the initial flight line and the second hillshade with the solar azimuth and zenith angles from the final flight line. The change in illumination was calculated by differencing the two hillshades.

The results from the differencing reviled that $99.4 \%$ of the scene experienced an increase in illumination, whereas only $0.4 \%$ and $0.2 \%$ of the scene experienced either a decrease in illumination or maintained the same illumination, respectively. An increase in illumination between $10-15 \%$ was experienced by $94 \%$ of the scene. The areas of the scene that experienced either a 
decrease in illumination or no change in illumination were restricted to olive groves, and built-up areas. The same is also true for the most extreme instances of illumination increases and decreases, which were also restricted to trees and built-up areas. This is because shadows were modeled in the hillshade and it is the trees and the built-up areas that generated the greatest shadow in the image. A histogram of the illumination change in the scene from the beginning of the flight campaign to the end of the flight campaign can be seen in Figure 4.6.

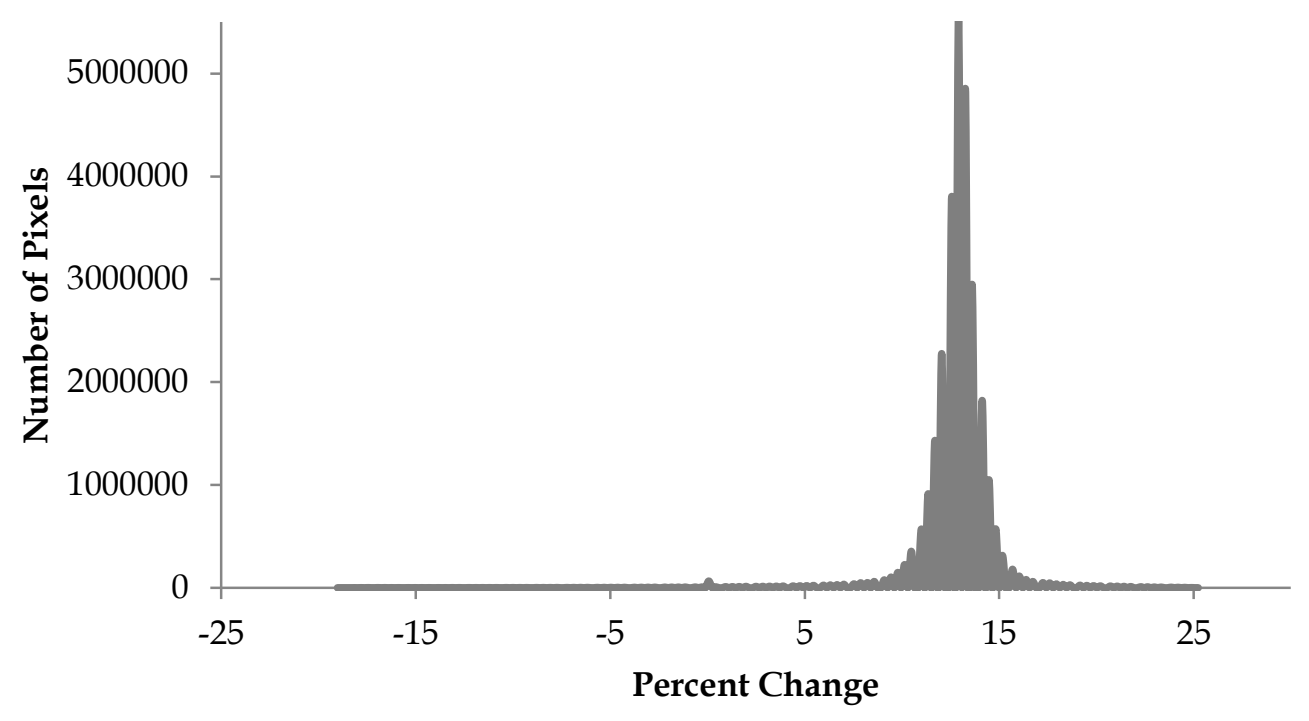

Figure 4.6: A histogram of the illumination change in the scene over the duration of the flight campaign. Negative values along the $\mathrm{X}$-axis represent a decrease in illumination and positive values along the $\mathrm{X}$-axis represent an increase in illumination. 
ATCOR underestimated the reflectance values for all six field locations relative to the ground spectra. A maximum of $30 \%$ absolute difference between field spectra and estimated ATCOR spectra was identified. These higher discrepancies in reflectance values were related with brighter surface covers. In contrast, darker surface covers were associated with smaller difference in reflectance values. It is believed that the underestimated surface reflectance values throughout the scene are the result of the overestimated visibility by ATCOR (Griffin and Burke, 2003).

At the 690-nm water vapour and oxygen absorption feature there is a $4 \%$ increase in the reflectance. This is followed by another $15 \%$ increase in reflectance at the 940-nm water absorption feature location. Both the $690 \mathrm{~nm}$ and $940 \mathrm{~nm}$ absorption features were interpolated in the atmospheric correction. The 1270-nm oxygen absorption feature was not corrected in the atmospheric correction and is still present in the data.

The empirical BRDF function in ATCOR was applied to the image. This function is intended for rough terrain and accounts for the BRDF effects as a result of the variations of the incident light beam (irradiance) (Richter and Schlapfer, 2012). However, the application of the empirical BRDF had no noticeable effects on the variations of the surface brightness within or between the flight lines. 
Validation of the atmospheric correction was conducted against spectra collected in the field. Although a substantial amount of noise was still present in the data, the overall spectral shape and the absorption features were comparable. For the validation, spectral signatures were gathered from the ATCOR results for the SU1 though SU6 field locations and were compared against the respective field spectra (Figure 4.7).

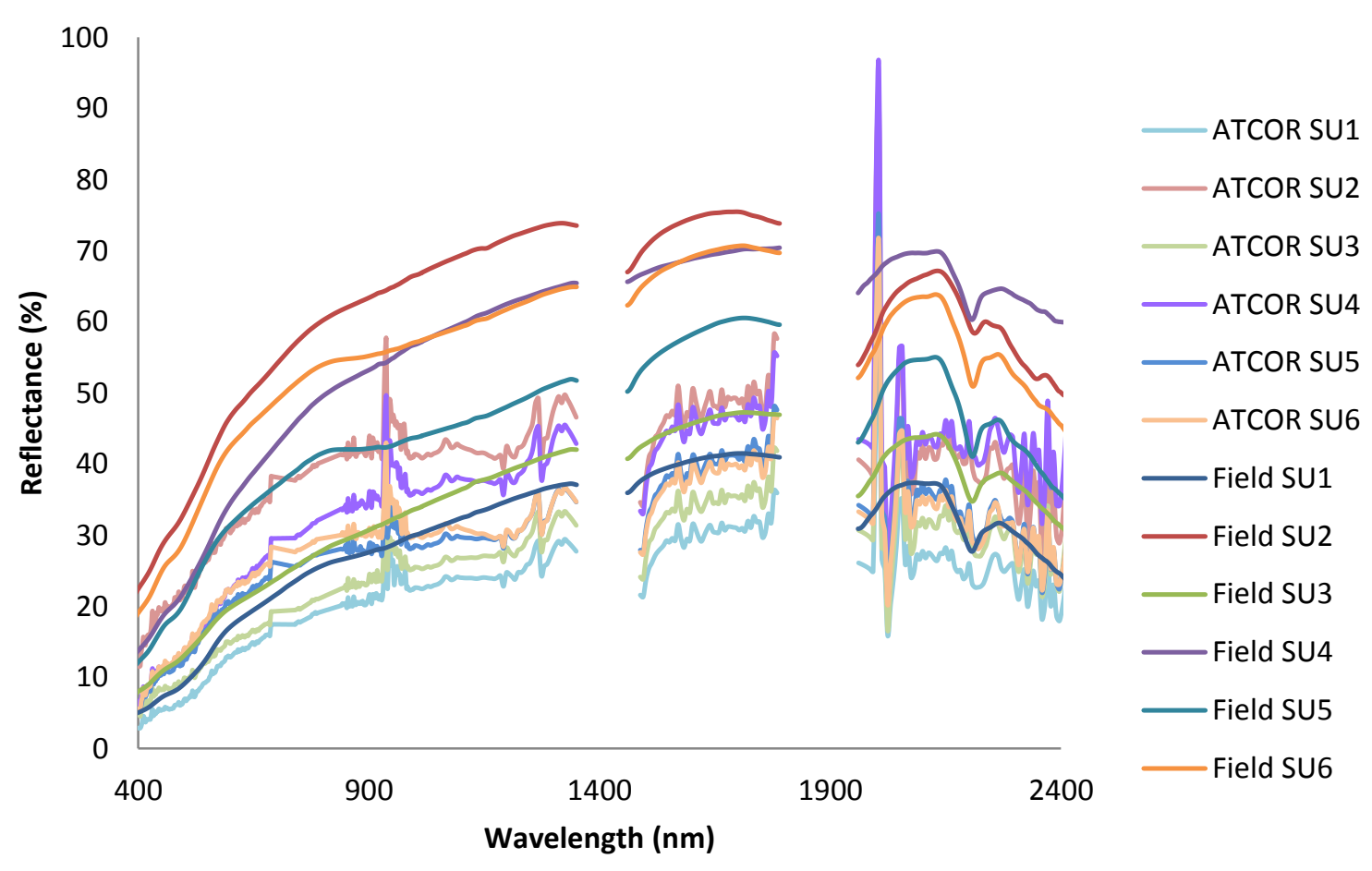

Figure 4.7: Comparison showing the difference in noise as well as overall reflectance values between the estimated ATCOR spectra and the field spectra for the SU1 through SU6 field sites. 
In all field locations, the 2210-nm clay absorption feature is present, but it has been shifted $10 \mathrm{~nm}$ to $2220 \mathrm{~nm}$. The $2349-\mathrm{nm}$ calcite feature in the SU2 field spectra is not visible in the ATCOR spectrum, either because it was not able to be resolved by the sensor or it has been masked by the substantial amount of noise present in the data from $2300 \mathrm{~nm}$ to $2400 \mathrm{~nm}$ (Figure 4.8).

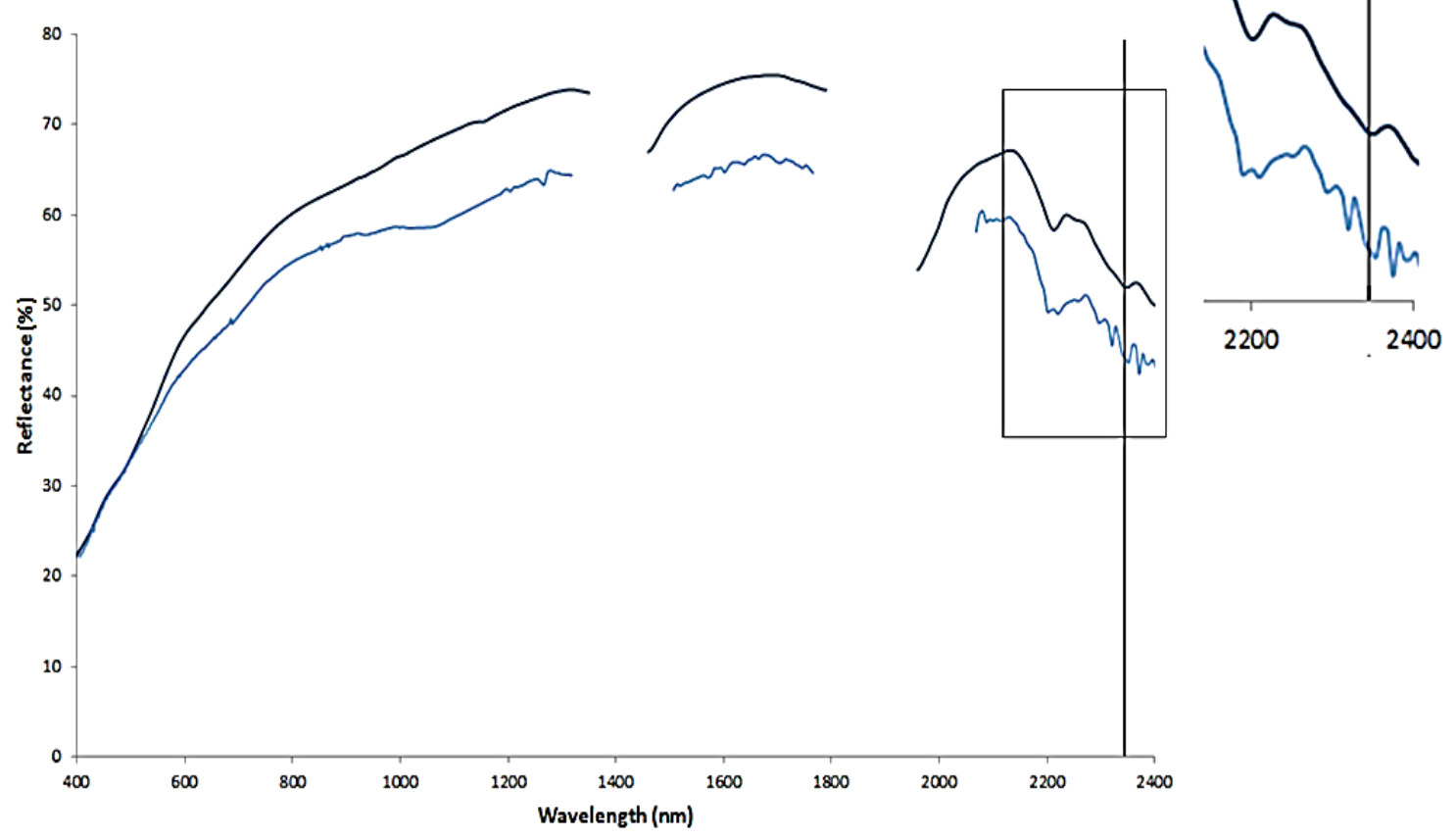

—Field-SU2 —AISA-SU2

Figure 4.8: Comparison of the field and AISA spectra from location SU2. The vertical line located at $2349 \mathrm{~nm}$ indicates the calcite feature present in the SU2 field spectra. The AISA spectra at this location contains a substaintial amount of noise and presents no clear calcite absorption feature. 
The spectral shift in the bands was further identified by comparing radiance spectra from the AISA data to an atmospheric absorption spectrum generated by an inverse application of MODTRAN. The MODTRAN spectrum was generated with a mid-latitude summer atmospheric model, rural aerosol model, 50-km visibility, default water content, sun zenith angle of 30 degrees, nadir viewing angle and $1-\mathrm{cm}^{-1}$ spectral resolution. Evaluating the extent of the shift was done by comparing the position of the atmospheric absorption features from the image spectrum to the positions in the MODTRAN spectrum (Neville et al., 2008). Comparison of the 760-nm oxygen absorption feature and the $940-\mathrm{nm}$ water absorption feature revealed no shift in the VNIR. Comparison of the $\mathrm{CO}_{2}$ features centered at $1570 \mathrm{~nm}, 2010 \mathrm{~nm}$ and $2060 \mathrm{~nm}$ revealed a shift of on average $7 \mathrm{~nm}$ towards the longer wavelengths in the SWIR. Figure 4.9 shows a comparison of the $\mathrm{CO}_{2}$ absorption features between the AISA data and the simulated MODTRAN spectrum. The spectral mis-calibration (shift) is the cause of the spikes and dips in the atmospheric absorption regions in the ATCOR derived surface reflectance (Richter and Schläpfer, 2012). 


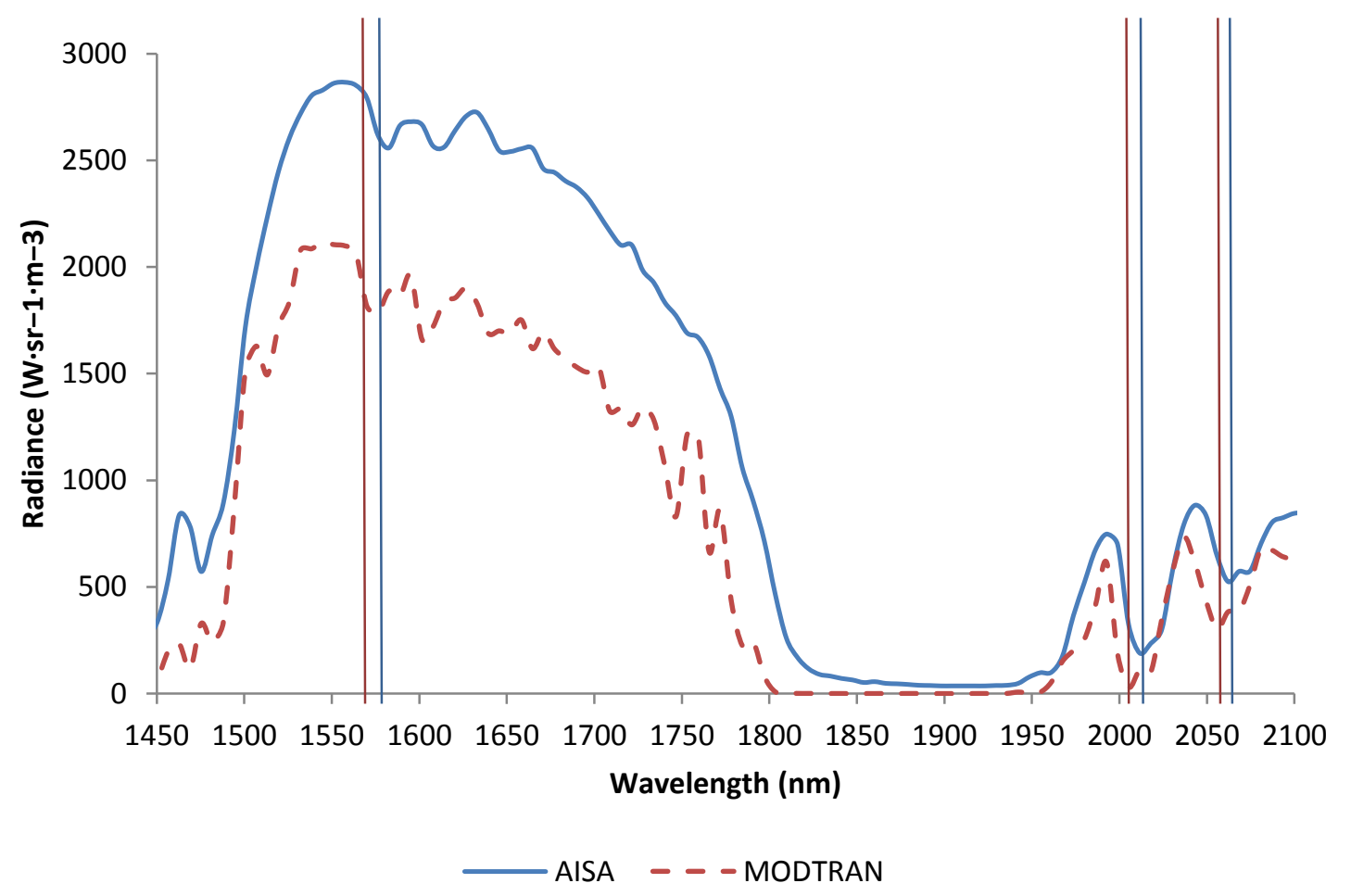

Figure 4.9: A comparison of the $\mathrm{CO}_{2}$ absorption features between the AISA data and the simulated MODTRAN spectrum. The AISA spectrum was collected from a road surface. The vertical lines in red and blue identify the center wavelength position of the MODTRAN and AISA $\mathrm{CO}_{2}$ absorption features, respectively.

The spectral shift could have been corrected for using the spectral calibration module offered in ATCOR-4. It is used to calculate new center wavelength positions and account for other non-uniformities, and modify the bands accordingly. This is to correct for any inconsistencies between the spectral calibration data and the actual center wavelength positions of the sensor since they often shift over time (Guanter et al., 2007; Green, 1998). 


\subsubsection{Cross-Track Illumination Correction}

The cross-track illumination correction was applied to the reflectance data in order to suppress brightness variation in the across-track (sensor nonuniformity) and along-track (BRDF) directions. Many different correction methods were attempted to compensate for the reflectance variations, including the empirical BRDF and nadir normalization in ATCOR. However, the results showed that the cross-track illumination correction was the most appropriate method.

The cross-track illumination correction was conducted following, and not before, the atmospheric correction because of the possible interference it may have with certain aspects of the atmospheric correction. This was a concern with parameters estimated directly from the image data such as water vapour and visibility. Although the cross-track illumination correction was able to reduce the effects of the across-track gradient, it was not able to remove them.

The quality of the correction was evaluated by comparing horizontal and vertical profiles from the same location in both the corrected and original image. Figure 4.10 shows an example of the resulting change in brightness in the acrosstrack direction before and after applying the cross-track illumination correction. In the image it can be seen that the cross-track illumination results normalized slightly the reflectance values on the edges of the flight line. 


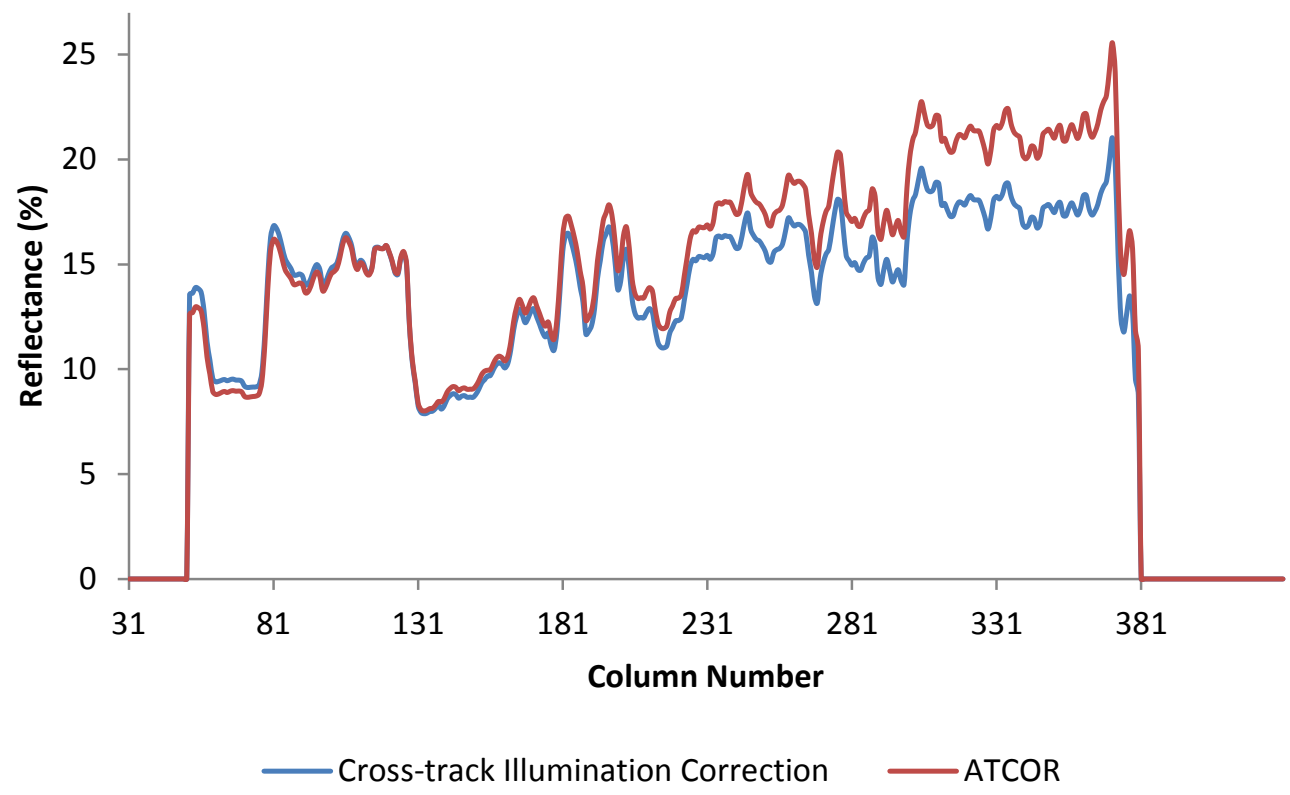

Figure 4.10: The x-profiles collected from the original ATCOR results and the cross-track illumination results. The x-profiles were collected from flight line 22004, band 64, line 3483.

\subsubsection{Mosaicking}

The mosaicking combined all of the individual flight lines into a single image. An example of the mosaicked scene can be seen in Figure 3.12. The data was cropped further to remove the uneven edges along the top and bottom of the image before further processing.

\subsubsection{Minimum Noise Fraction}

A MNF consists of two cascading PCAs and was used in this thesis to segregate the noise component and ultimately reduce the dimensionality of the 
data. The first $38 \mathrm{MNF}$ components out of a total of 486 bands were selected as coherent and were used in an inverse MNF.

\subsubsection{Empirical Line Correction}

An ELC was applied to the combined AISA Eagle and Hawk image data to remove residual atmospheric absorption features remaining from the atmospheric correction. The results of the ELC were still periodically noisy, but the remaining atmospheric absorption features were removed. It also resulted in clearer spectral shapes and better definition of the distinguishing soil mineral absorption features (Figure 4.11).

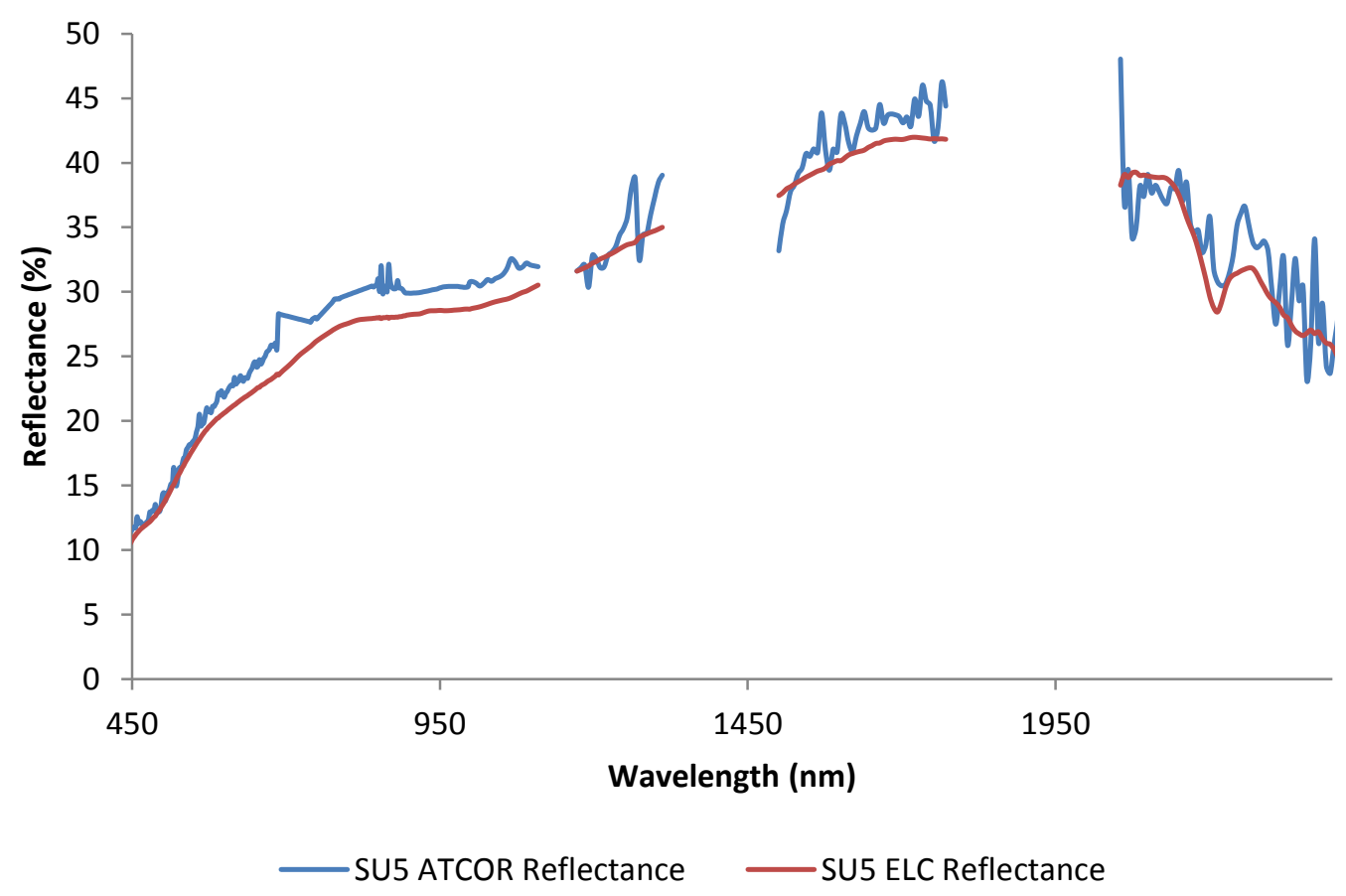

Figure 4.11: Comparison between the ATCOR spectrum and the corresponding ELC spectrum for field location SU5. 
Negative values were introduced into the data as a result of the ELC. This is a common outcome, which occurs when the dark target selected for the ELC is not dark enough, and as a result the offset term becomes negative. An example can be seen with the reflectance values at $1456.5 \mathrm{~nm}$. In this instance, any reflectance values below $11.5 \%$ will result in negative values in the ELC output (Figure 4.12).

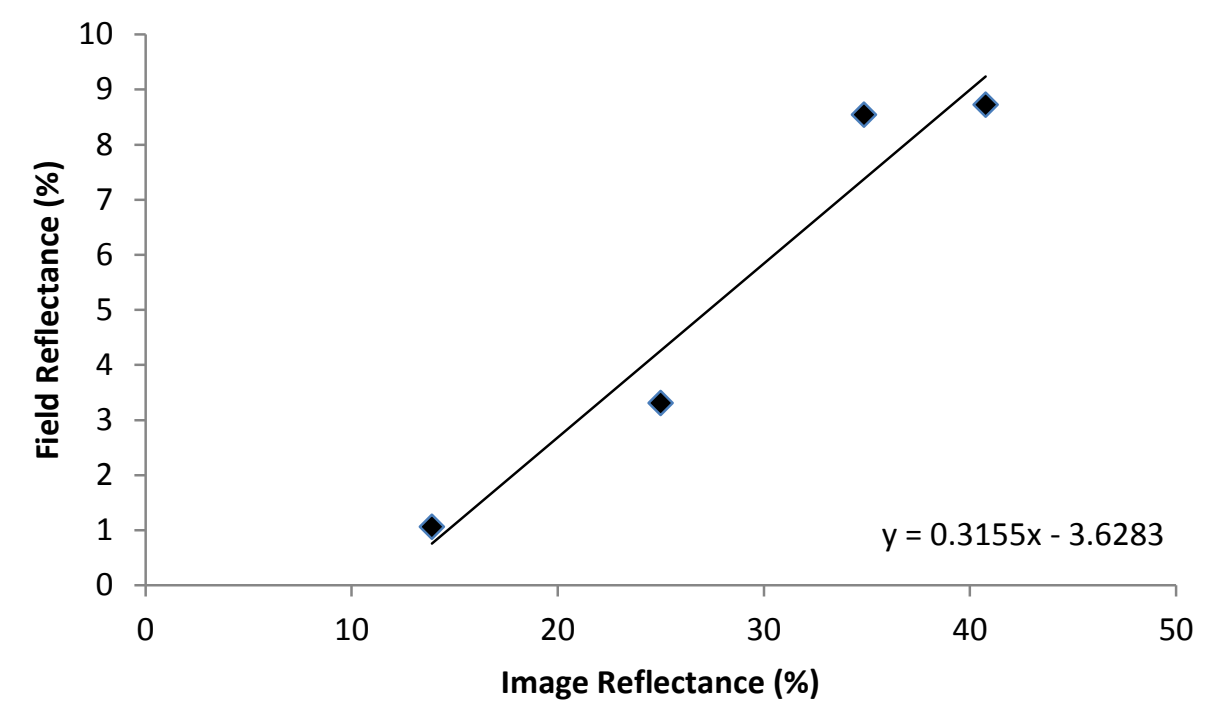

Figure 4.12: The ELC plot for $1456.5 \mathrm{~nm}$. Each of the points represents one of the four spectra used for the ELC.

Because the negative values occurred in only the dark surface cover (dense green vegetation, burned fields, etc.), there was little interference with the bare soil cover. Nevertheless, the negative values were masked in both the AISA and 
simulated EnMAP data so that they would not affect further processing or statistics.

\subsection{EnMAP Simulation}

The EnMAP simulation produced a simulated EnMAP reflectance cube from the pre-processed combined AISA Eagle and Hawk data described in the previous sections. The EnMAP data were simulated to the BOL spatial, spectral and radiometric characteristics of the sensor. In comparison to the original AISA data, there was a decrease in both spatial resolution, from $6 \mathrm{~m}$ to $30 \mathrm{~m}$, and in spectral resolution, from 486 bands to 244 bands.

The only post-processing step conducted on the simulated EnMAP data was the removal of the sensor overlap. The sensor overlap occurred between 900 $\mathrm{nm}-1000 \mathrm{~nm}$ where both the VNIR sensor and the SWIR sensor collect radiance. Figure 4.13 shows the sensor overlap. The SWIR signal within the sensor overlap region was kept due to its greater sensitivity (quantum efficiency) in the $900 \mathrm{~nm}$ to $1000 \mathrm{~nm}$ range. A comparison of the AISA data and the simulated EnMAP data can be seen in Figure 4.14. 

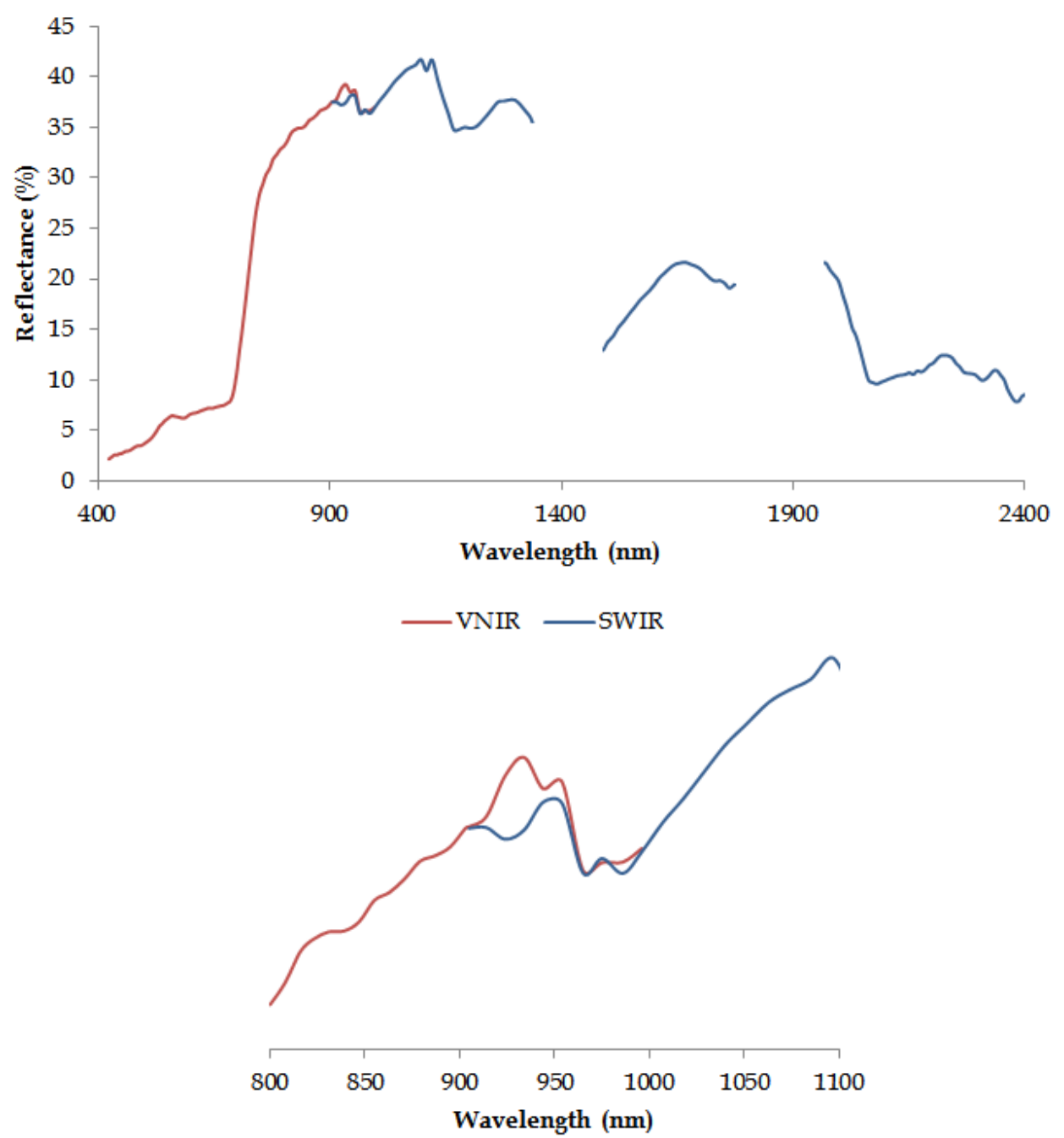

Figure 4.13: The contribution of the VNIR and SWIR detectors to the image spectra (top). A zoom in on the detector overlap region (bottom). 

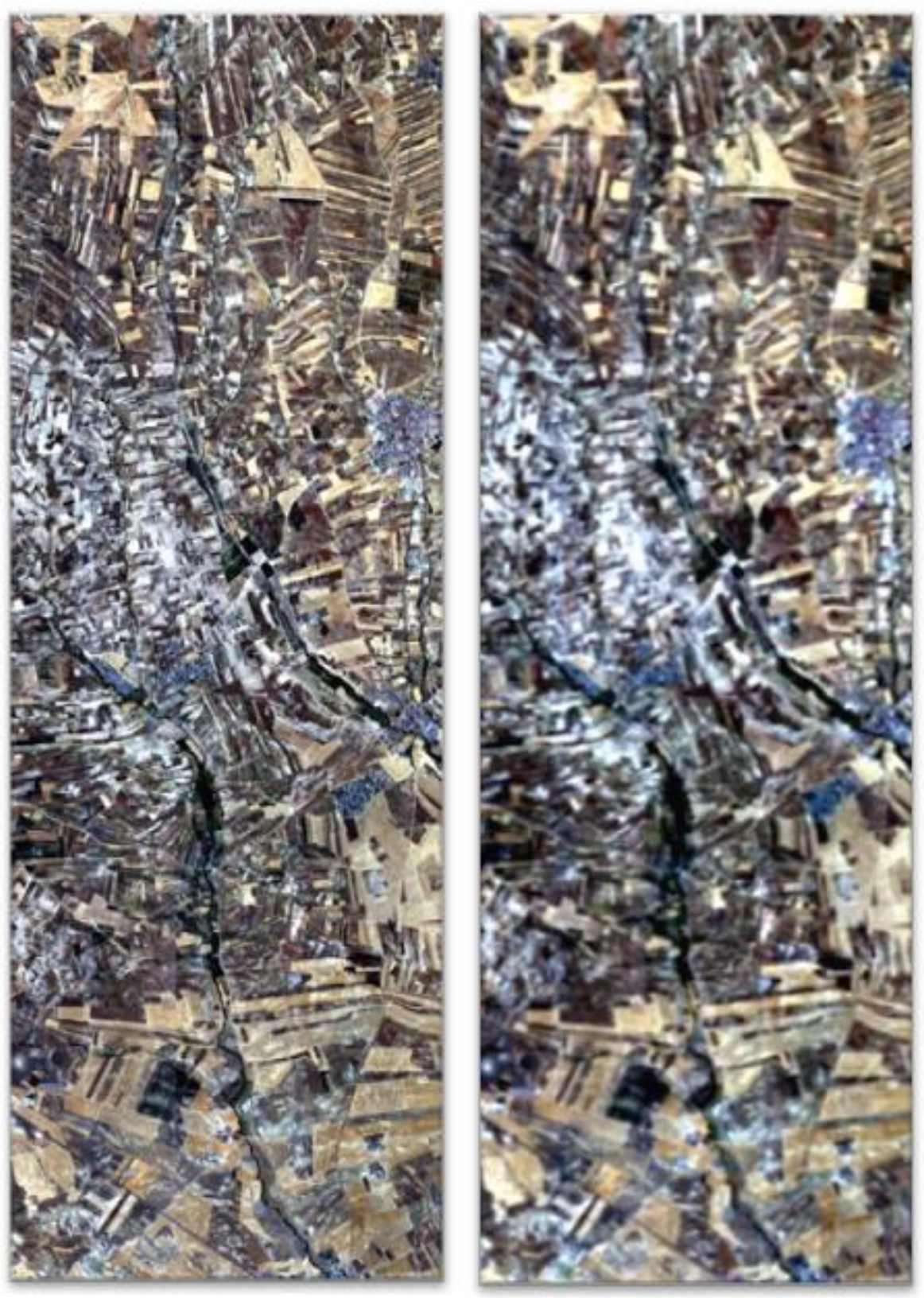

Figure 4.14: Images of the AISA data (6 m; left) and the simulated

EnMAP data (30 m; right) (RGB composite R: 640 nm, G: 550 nm, B: 460

$\mathrm{nm})$ to compare the spectral resolutions between the two data sets. 
Two spectral irregularities were introduced into the data by the simulation. The first irregularity manifest itself as 'red' vegetation in densely vegetated areas. This can be seen in Figure 4.15. This spectral irregularity was found to be a result of the negative values generated in the ELC. All negative values in the AISA data and the corresponding pixels in the EnMAP data were masked before further processing.
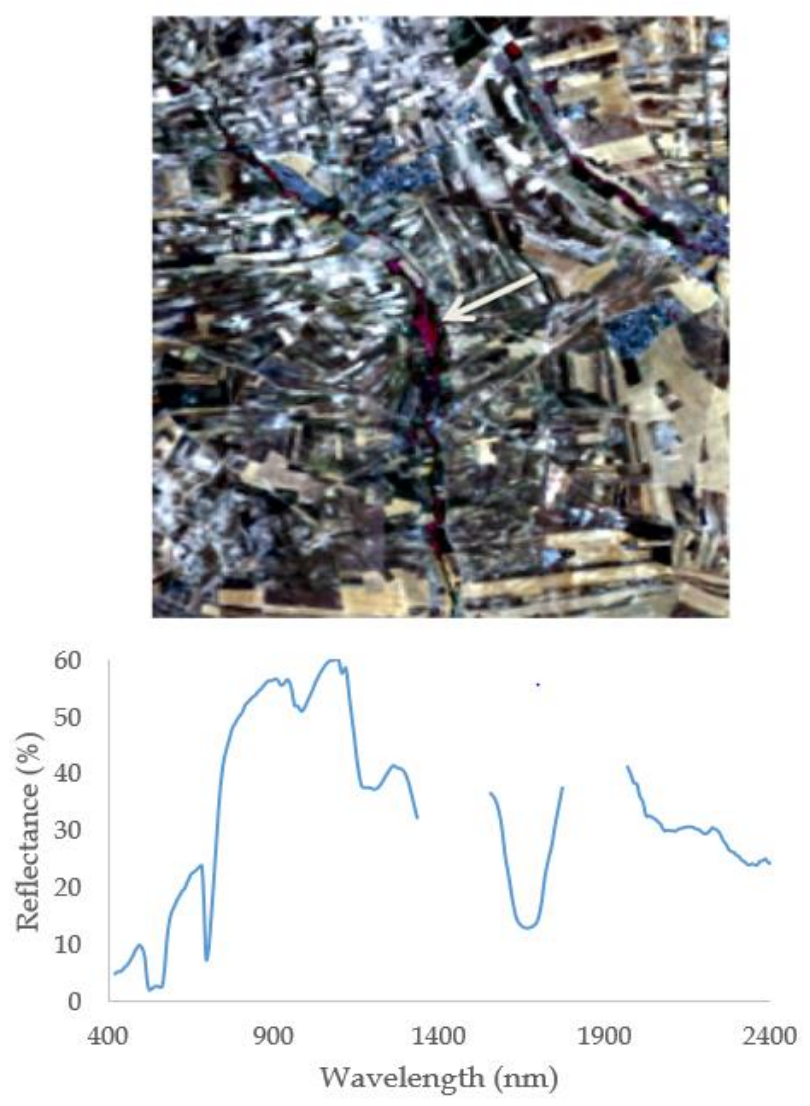

Figure 4.15: An example of the 'red' vegetation in the simulated EnMAP data

(top) (RGB composite; R: 640 nm, G: $550 \mathrm{~nm}, \mathrm{~B}: 460 \mathrm{~nm}$ ) and the spectral signature associated with the 'red' vegetation (bottom). 
The second spectral artifact introduced by the EnMAP simulation was an overestimation of the reflectance in the water vapour absorption features located at $940 \mathrm{~nm}$ and $1130 \mathrm{~nm}$. The water vapour overestimation artifacts and the associated spatial pattern can be seen in Figure 4.16. The wavelength regions containing the artifacts, between $905 \mathrm{~nm}-975 \mathrm{~nm}$ and $1085 \mathrm{~nm}-1179 \mathrm{~nm}$, were removed because no effective method could be found to compensate for the overestimation within these features.

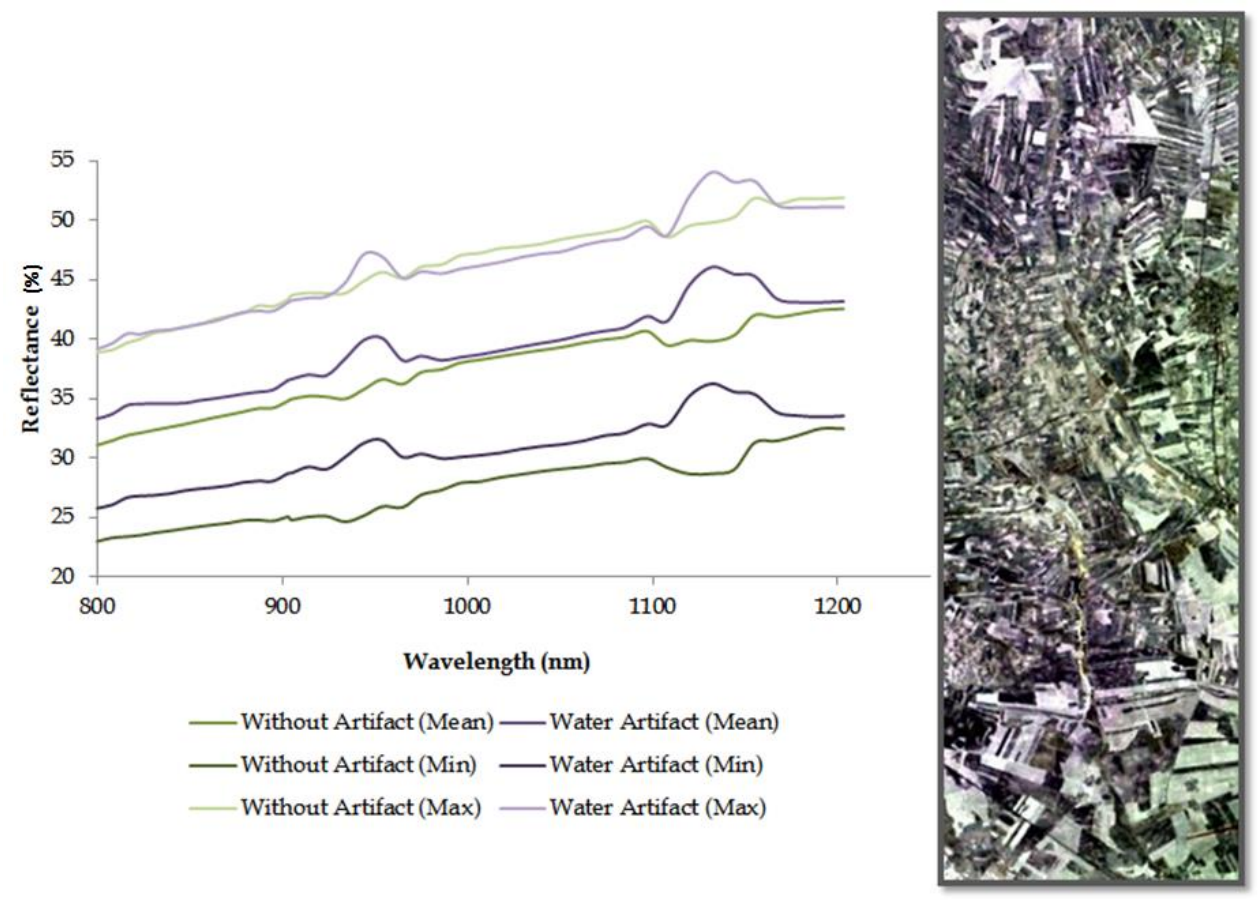

Figure 4.16: Purple areas contain the water vapour overestimation artifacts. Green areas do not exhibit the water vapour overestimation artifact. The figure on the left shows the maximum, minimum and mean reflectance of the Region of Interest (ROI) collected from both artifact affected and non-artifact affected areas. RGB image on the right; Red $=944$ nm, Green $=1040$ nm, Blue $=1132$ nm. 


\subsection{Soil Mapping}

The purpose of image processing is to extract information from the image data. The image processing aimed to derive a soil erosion state map from the simulated EnMAP data and generate a validation image from the pre-processed combined AISA Eagle and Hawk data. The image processing was conducted only on the SU study site subset, unlike the data processing in sections 4.1 and 4.2, which were conducted on the entire Camarena site. Results are provided for the three main image processing steps: 1) the masking of green and dry vegetation, 2) the classification of soil erosion states and, 3) the validation. The results for each of the main processing steps are separated into two sections, the results obtained from the AISA image processing and the results obtained from the EnMAP image processing.

\subsubsection{Vegetation Masks}

The vegetation masks were generated from MESMA soil, green vegetation and dry vegetation fraction images. The results presented focus mainly on the fraction and RMSE images used to generate the masks, because the masks themselves are just a representation of the MESMA output.

Out of the total 288 models created from the four endmembers, 281 models were applicable within the AISA scene. The largest proportion of the scene characterized by a single model was $9.5 \%$. This model accounted for nearly one tenth of the scene out of a total of 281 models. The need for 281 
models to fully unmix the scene indicates that there is a substantial amount of small scale variability within the scene. This small scale variability could be a result of environmental or sensor, internal or procedural influences, such as noise or spectral miscalibration.

All of the pixel fractions in the AISA image sum to unity, which is default for the MESMA unmixing. However, non-real fractions accounted for approximately $4 \%$ and $7 \%$ for the dry vegetation and soils endmembers, respectively. The green vegetation endmember had negative fraction values for approximately $26 \%$ of the scene and contained no fraction values above one. Non-real in this instance refers to fraction values below zero and above one. One possible reason for this occurring is that a pixel within the ASIA image remains that is purer than the endmember pixels selected for the unmixing (Darvishsefat et al., 2002). The non-real fraction values can also indicate that either the endmembers chosen were not adequate for the unmixing or that there are additional endmembers missing from the model (Exelis, 2014b). Table 4.1 shows how deviant each endmember was from 'real' fraction values $(0$ - 1$)$.

Figure 4.17 shows the RMS error image from the unmixing. Table 4.2 presents the RMS error, classified into ranges selected from Haboudane et al. (2002), and Figure 4.18 shows a histogram of the RMS error. 
Table 4.1: Percent of the total AISA scene that has fraction values below zero and above one for each endmember.

\begin{tabular}{|c|c|c|}
\hline \multicolumn{3}{|c|}{ Reality Constraints } \\
\hline Endmember Class & $\begin{array}{c}\text { Fractions }<0 \\
(\%)\end{array}$ & $\begin{array}{c}\text { Fractions }>1 \\
(\%)\end{array}$ \\
\hline Green Vegetation & 26.3 & 0 \\
\hline Dry Vegetation & 1.4 & 2.4 \\
\hline Soils & 3.4 & 3.5 \\
\hline
\end{tabular}

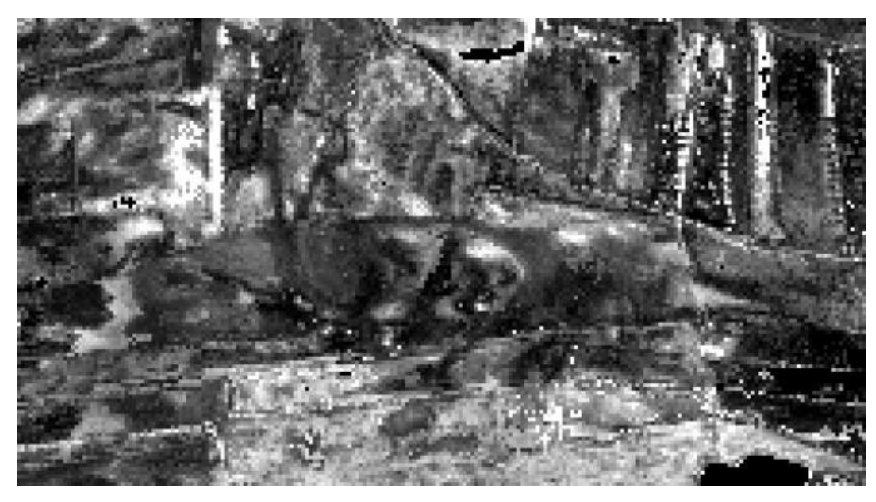

Figure 4.17: RMS error image from the AISA vegetation unmixing. Brighter pixels represent higher RMS error values. There are pixels where the endmember models were unable to accurately unmix the pixel spectra. 
Table 4.2: Percent of the RMS error image that falls in to each class. The class values are selected based on Haboudane et al. (2002).

\begin{tabular}{|c|c|}
\hline $\begin{array}{c}\text { RMSE } \\
\text { Class }\end{array}$ & $\%$ \\
\hline $0-0.5$ & 13.82 \\
\hline $0.5-1.5$ & 68.20 \\
\hline $1.5-5$ & 17.87 \\
\hline$>5$ & 0.09 \\
\hline
\end{tabular}

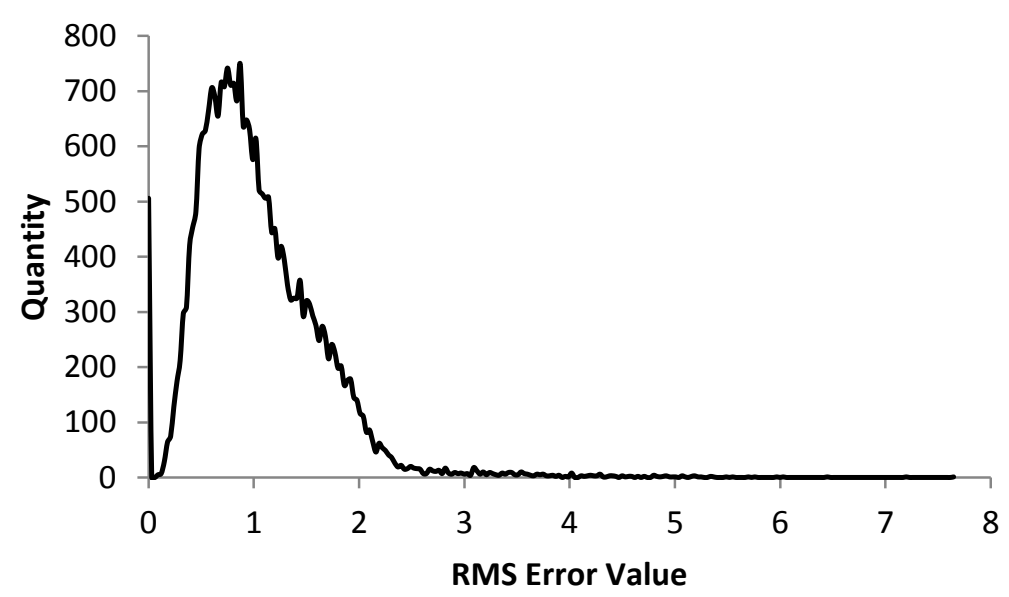

Figure 4.18: Histogram of the RMS error values from the AISA unmixing.

The vegetation mask was validated against the six known field locations within the scene, because all field locations represented locations of bare soil exposed at the surface. The validation consisted of identifying whether any of the points resided beneath the vegetation mask, which was not the case in this study (Figure 4.19). 


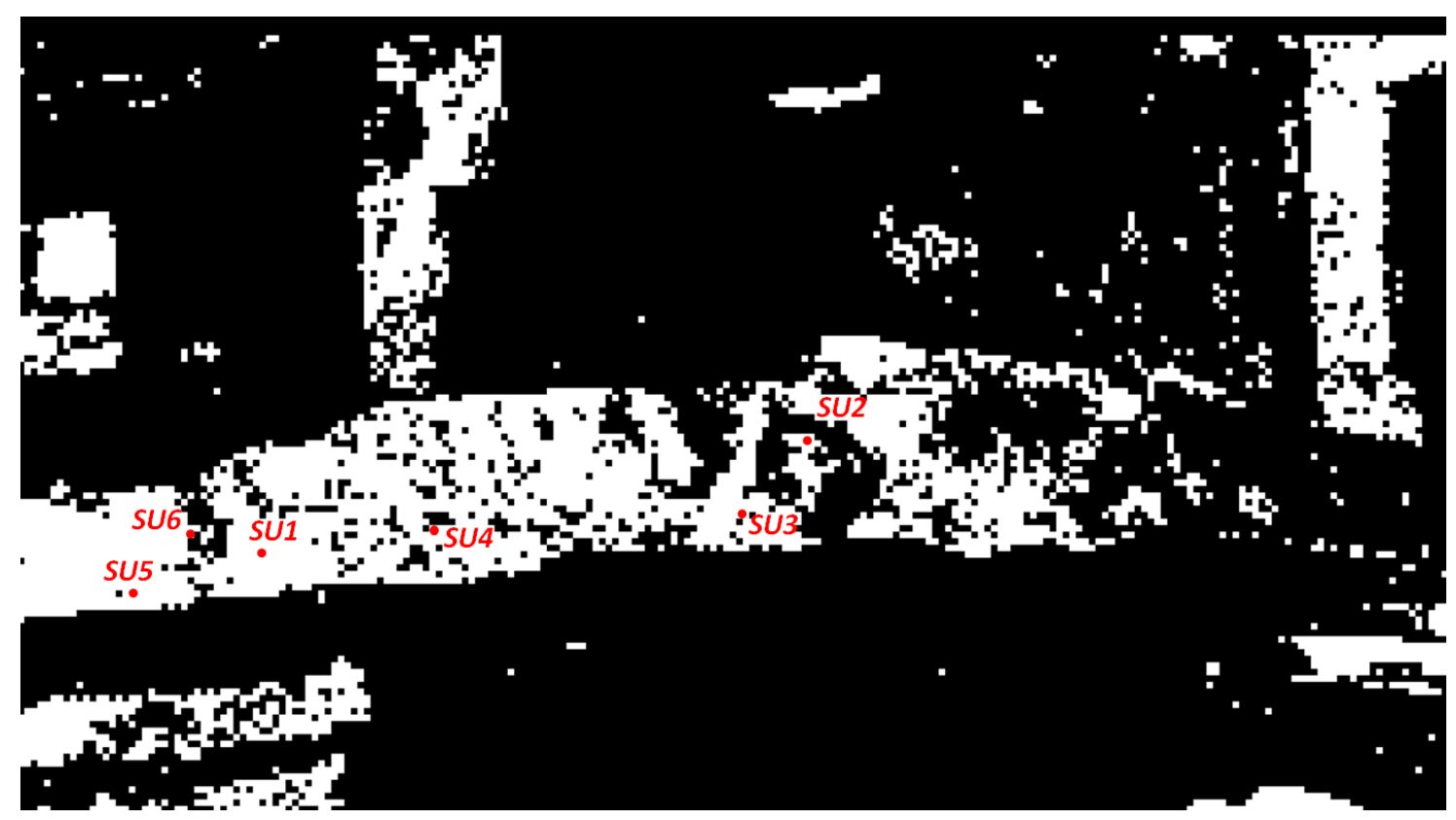

Figure 4.19: Vegetation mask generated from the AISA data. The black pixels compose the mask. All six SU sites (highlighted in red) do not reside under this mask.

Out of the total 484 models created from the 4 endmembers used to unmix the simulated EnMAP image, only 277 models were applicable within the scene resulting in $5.9 \%$ of the scene unclassified. The large proportion of the scene that remained unclassified gives an indication of scene complexity. The largest proportion of the scene characterized by a single model was $2.9 \%$. 
In accordance with the AISA results, the pixels in the image sum to unity. However, the total non-real fractions for the dry vegetation and soils endmembers were approximately $4 \%$ and $6 \%$, respectively. Likewise, the green vegetation endmember had negative fraction values much higher than was recorded for the other two endmembers. Approximately $35 \%$ of the green vegetation fraction image contained negative values, with no fraction values exceeding one. Table 4.3 shows the deviation of each simulated EnMAP endmember from real fraction values.

Table 4.3: Percent of the total EnMAP scene that has fraction values below zero and above one for each endmember.

\begin{tabular}{|l|l|l|}
\hline \multicolumn{3}{|c|}{ Reality Constraints } \\
\hline Endmember Class & $\begin{array}{l}\text { Fractions }<0 \\
(\%)\end{array}$ & $\begin{array}{l}\text { Fractions }>1 \\
(\%)\end{array}$ \\
\hline Green Vegetation & 35.0 & 0 \\
\hline Dry Vegetation & 2.4 & 1.8 \\
\hline Soils & 5.0 & 1.2 \\
\hline
\end{tabular}

$84.5 \%$ of the RMS error remained below 1.5 with no error values exceeding 5. Figure 4.20 shows the RMS error image for the EnMAP unmixing. The classified RMS error values can be seen in Table 4.4, and a histogram of the RMS error is presented in Figure 4.21. The low RMS error values seen in Table 4.4 and Figure 4.22 mean that there was a good fit between the endmember models selected for the unmixing and the mixed signal from each pixel. 


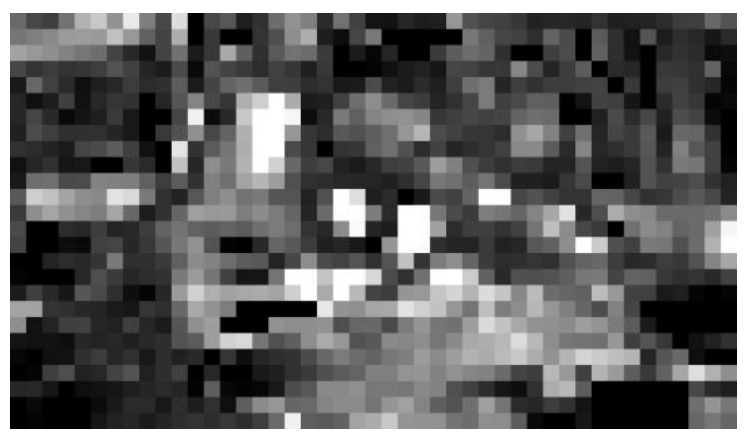

Figure 4.20: RMS error image from the simulated EnMAP unmixing. Brighter pixels represent higher RMS error values. There are pixels where the endmember models were unable to accurately unmix the pixel spectra.

Table 4.4: Percent of the RMS error image that falls in to each class. The class values are selected based on Haboudane et al. (2002).

\begin{tabular}{|c|c|}
\hline $\begin{array}{c}\text { RMSE } \\
\text { Class }\end{array}$ & \% \\
\hline $0-0.5$ & 17.3 \\
\hline $0.5-1.5$ & 67.3 \\
\hline $1.5-5$ & 15.4 \\
\hline$>5$ & 0 \\
\hline
\end{tabular}

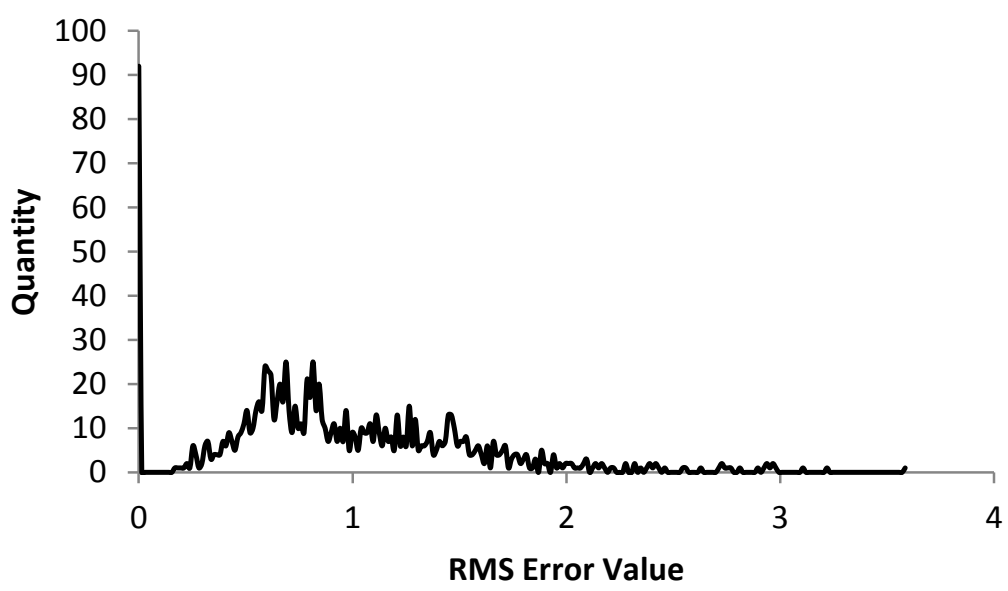

Figure 4.21: The histogram of RMS error values from the EnMAP unmixing. 
Due to the different spatial scales between the simulated EnMAP data and the field data collection, the method used to validate the AISA data was not applicable to the simulated EnMAP data. The simulated EnMAP vegetation mask was visually examined against the AISA vegetation mask to identify whether similar patterns were expressed in both. The two masks did exhibit similar patterns; nevertheless, considerable differences exist between the two masks due to the difference in the GSD.

\subsubsection{Soil Erosion Status Classification}

The soil classification was conducted for the purpose of identifying soil erosion stages within the study area. MESMA fraction images were used as the basis for the soil classification. The soil classification results for the AISA and the simulated EnMAP images will focus on the MESMA output as well as the final soil erosion state classification map.

Three-endmember models were used for both the AISA and the simulated EnMAP unmixing procedures. The endmembers applied were eroded, accumulated and shade. The accumulated and eroded fractions were shade normalized prior to classification, which in essence eliminates the effects of illumination, soil moisture and surface roughness variation (Hill et al., 1994).

A total of 16 models were created from the three endmembers selected for the AISA scene. All 16 models were used to unmix the AISA scene with $43.7 \%$ 
being the largest proportion of the scene characterized by a single model. The 15 other models ranged from $15.8 \%$ to less than $1 \%$ of the scene.

All of the pixels in the image sum to unity however, non-real fractions accounted for approximately $3 \%$ for both the accumulated and eroded fractions. The non-real fraction results can be seen in Table 4.5. 86 \% of the RMSE falls below 1.5 with less than one percent exceeding $5 \%$.The categorized RMS error for the unmixing is listed in Table 4.6.

Table 4.5: Percent of the total AISA scene that has fraction values below zero and above 1 for each endmember in the soils unmixing.

\begin{tabular}{|c|c|c|}
\hline \multicolumn{2}{|c|}{ Reality Constraints } & \\
\hline Endmember & Fractions $<0(\%)$ & Fractions $>1(\%)$ \\
\hline Accumulated & 0.7 & 2.7 \\
\hline Eroded & 2.8 & 0.6 \\
\hline
\end{tabular}

Table 4.6: Percent of the RMS error image that falls in to each class for the AISA soils unmixing. The class values are selected based on Haboudane et al. (2002).

\begin{tabular}{|c|c|}
\hline $\begin{array}{c}\text { RMSE } \\
\text { Class }\end{array}$ & \% \\
\hline $0-0.5$ & 81.6 \\
\hline $0.5-1.5$ & 4.2 \\
\hline $1.5-5$ & 14.3 \\
\hline$>5$ & 0.1 \\
\hline
\end{tabular}


The validation of the AISA unmixing was conducted against the four field locations not included in the endmember selection (SU1, SU3, SU5, SU6). Table 4.7 shows the eroded and accumulated fraction values calculated for each field site.

Table 4.7: Eroded and accumulated fraction values from each field location within the AISA image.

\begin{tabular}{|c|c|c|}
\hline & \multicolumn{2}{|c|}{ Fraction Values } \\
\hline Field Sampling Site & Eroded & Accumulated \\
\hline $\begin{array}{c}\text { SU2 } \\
\text { Most Advanced Erosion }\end{array}$ & 0 & 1 \\
\hline $\begin{array}{c}\text { SU6 } \\
\text { Advanced Erosion }\end{array}$ & 0.07 & 0.93 \\
\hline $\begin{array}{c}\text { SU5 } \\
\text { Advanced Erosion }\end{array}$ & 0.29 & 0.7 \\
\hline $\begin{array}{c}\text { SU1 } \\
\text { Intermediate } \\
\text { SU3 } \\
\text { Intermediate }\end{array}$ & 0.52 & 0.48 \\
\hline $\begin{array}{c}\text { SU4 } \\
\text { Accumulation }\end{array}$ & 0.79 & 0.21 \\
\hline
\end{tabular}

In the final AISA classification map, 941 pixel of a total 1032 pixels in the scene, or $91.2 \%$, were masked. It includes masking of the negative values, the vegetation mask and the additional masking of pixels that fell outside the overlapping region with the simulated EnMAP data. This is equivalent to 0.84 $\mathrm{km}^{2}$, leaving only $0.08 \mathrm{~km}^{2}$ of the original scene for the soil classification. Of the remaining $8.8 \%$ of the scene, the eroded class accounted for $13 \%$, the 
accumulated class for $18 \%$ and the intermediate class for $69 \%$. The final 30-m AISA soil erosion state validation map can be seen in Figure 4.22.

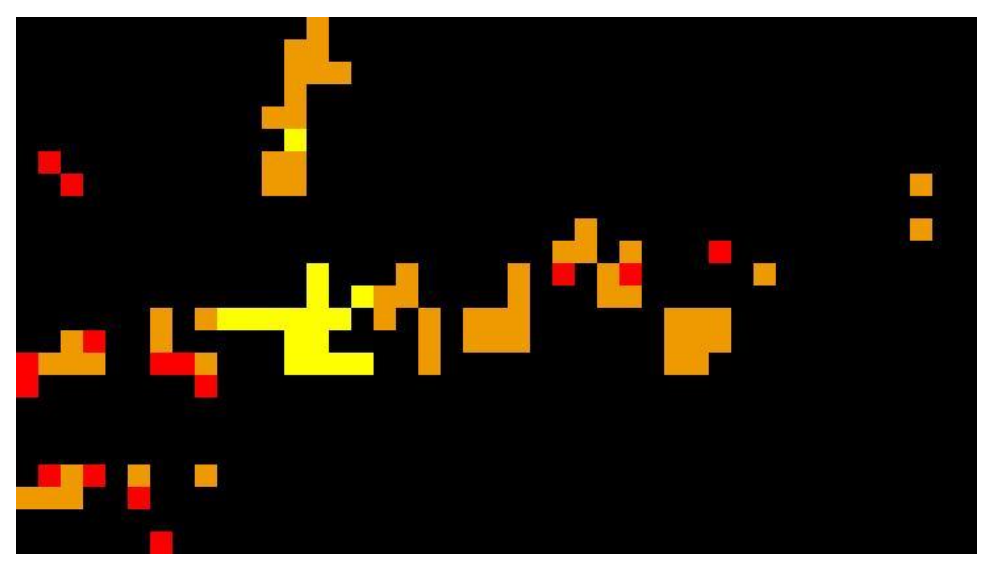

Figure 4.22: Final 30-m AISA soil erosion state validation map. Yellow $=$ accumulated, orange $=$ intermediate and red $=$ eroded.

Two models were created from the three endmembers selected for the EnMAP scene. They accounted for $48 \%$ and $9 \%$ of the scene, respectively. $43 \%$ of the scene remained unclassified. There was a high percentage of unclassified pixels, because of unidentifiable soil spectra. For example, an area located in the northern portion of the subset remained largely unclassified, because it had no other similarities to the other soils in the scene besides containing the $2200-\mathrm{nm}$ clay absorption feature. Although these unclassified pixels could have been avoided by collecting an endmember spectrum from the region, there was no indication, spectrally or topographically, as to whether the area could be considered accumulated or eroded. The soil spectral signature representative of this area is shown in Figure 4.23. Topographically, the area displayed properties 
characteristic of both eroded (convex) and accumulated sites (concave). Figure 4.24 shows elevation profiles of the area.

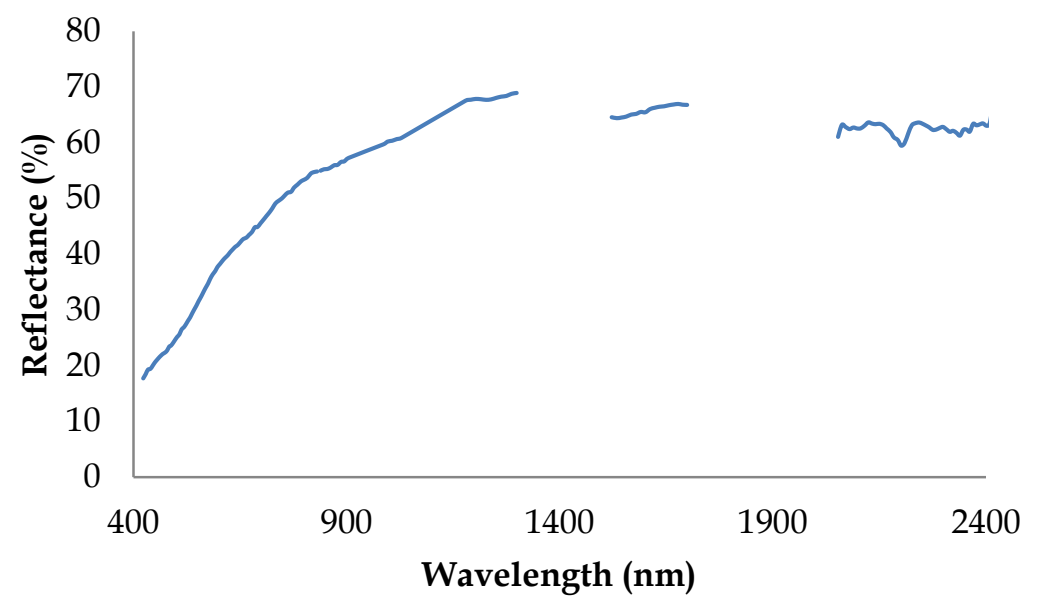

Figure 4.23: Example of unidentifiable spectra, which remained unclassified in the soils unmixing of the EnMAP data. 


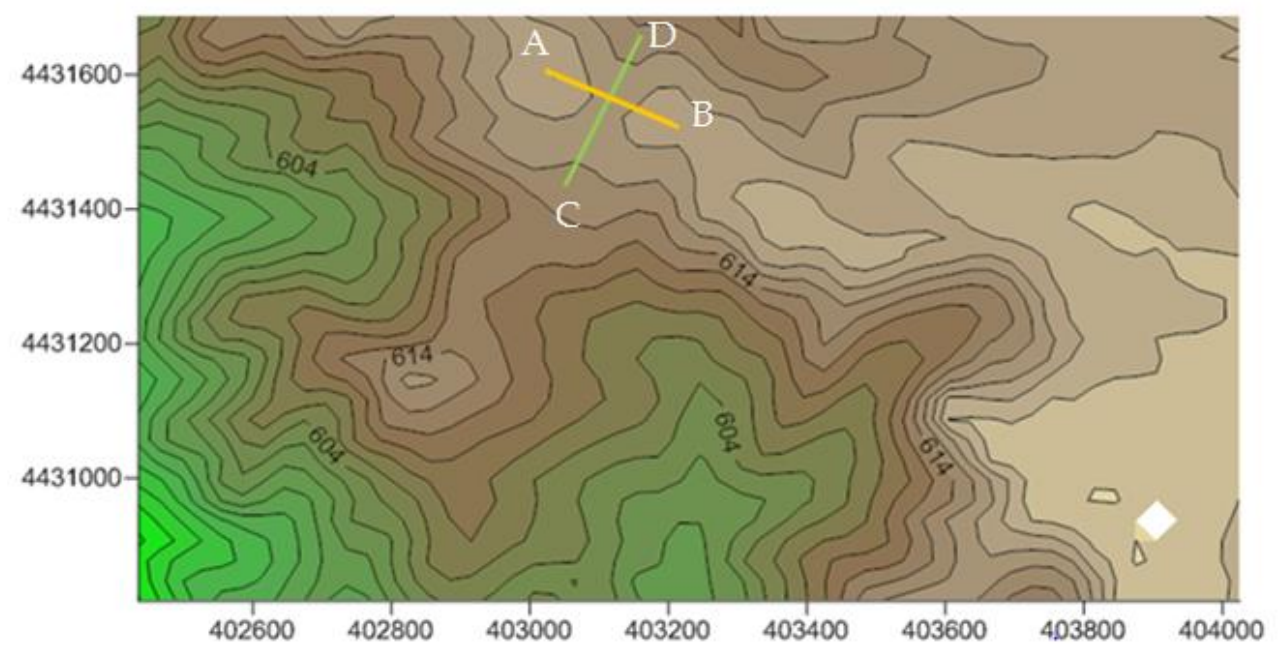

A

B
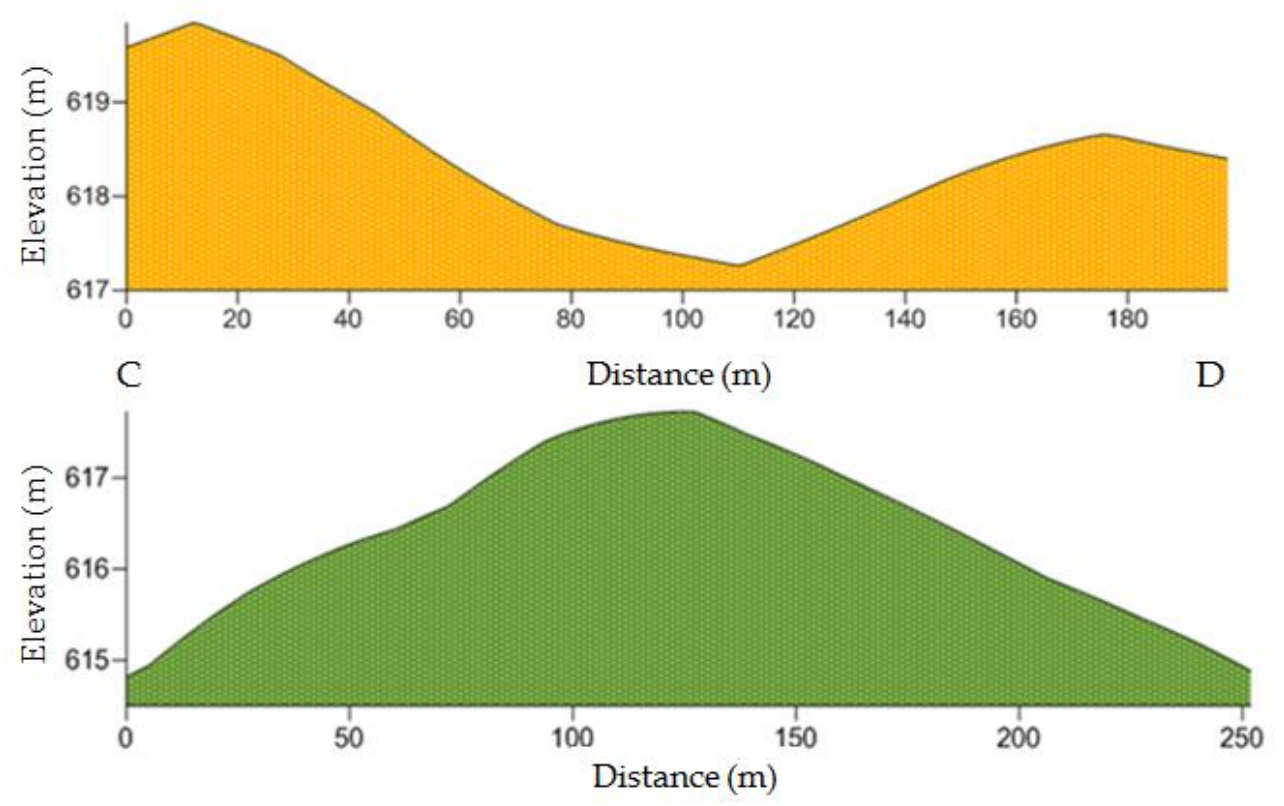

Figure 4.24: Elevation profiles from a poorly identified region in the EnMAP unmixing. 
All of the pixel fractions in the simulated EnMAP image sum to unity; however, non-real fractions accounted for approximately $12 \%$ for both the accumulated and eroded fractions. The non-real fraction results are presented in Table 4.8. $89 \%$ of the RMSE falls below 1.5 with no pixels exceeding $5 \%$. The categorized RMS error for the unmixing is listed in Table 4.9.

The EnMAP final classification map also had $91.2 \%$ of the scene masked. This is because both the 30-m AISA and simulated EnMAP classifications needed to cover the same extent for the confusion matrix.

Table 4.8: Percent of the total scene that has fraction values below zero and above 1 for each endmember in the EnMAP soils unmixing.

\begin{tabular}{|l|c|c|}
\hline \multicolumn{3}{|c|}{ Reality Constraints } \\
\hline Endmember & $\begin{array}{c}\text { Fractions }<0 \\
(\%)\end{array}$ & $\begin{array}{c}\text { Fractions }>1 \\
(\%)\end{array}$ \\
\hline Accumulated & 11.3 & 0.6 \\
\hline Eroded & 1.9 & 9.4 \\
\hline
\end{tabular}

Table 4.9: Percent of the RMS error image that falls in to each RMSE class for the EnMAP soils unmixing. The class values are selected based on Haboudane et al. (2002).

\begin{tabular}{|c|c|}
\hline $\begin{array}{c}\text { RMSE } \\
\text { Class }\end{array}$ & \multicolumn{1}{c|}{} \\
\hline $0-0.5$ & 86.7 \\
\hline $0.5-1.5$ & 2.77 \\
\hline $1.5-5$ & 10.6 \\
\hline$>5$ & 0 \\
\hline
\end{tabular}


Of the remaining scene, the eroded class accounted for $16 \%$, the accumulated class for $18 \%$ and the middle class for $66 \%$. The final soil state classification map can be seen in Figure 4.25. The accumulated class was equally represented in both the 30-m AISA and the simulated EnMAP classifications. The latter had more pixels classified as eroded and accordingly, less pixels classified as intermediate than the 30-m AISA classification.

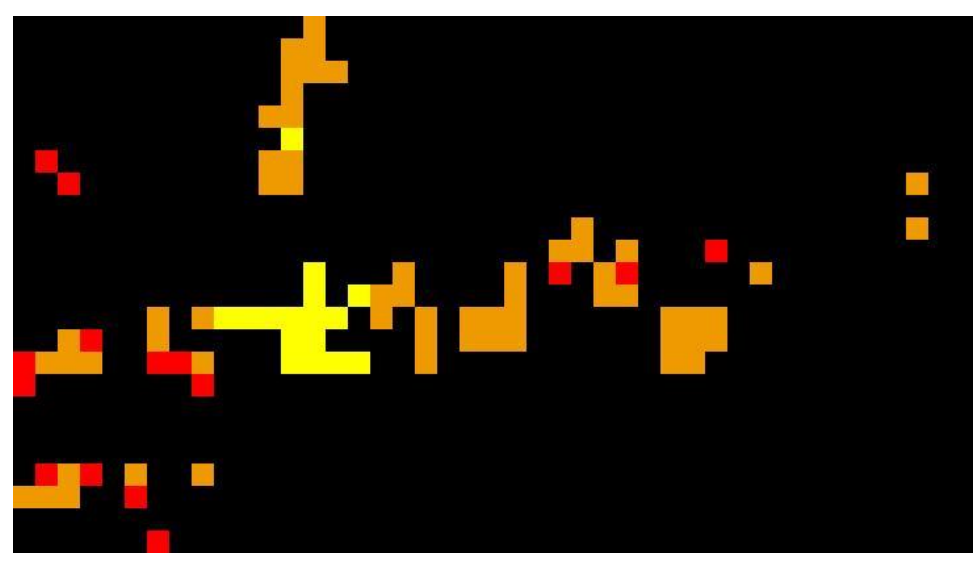

Figure 4.25: Final EnMAP soil erosion state map. Yellow $=$ accumulated, orange $=$ intermediate and red $=$ eroded .

Effects of the remaining BRDF effect and non-uniformity were seen in the soil erosion classification results particularly when comparing the two mosaicked flight lines that make up the scene. The subsetting of the flight lines prior to mosaicking and the small area in which this research is focused (SU site) would both have reduced the overall influence of the BRDF and non-uniformity effects 
compared to if the research had been conducted on a larger area of the Camarena study site.

\subsubsection{Validation}

Validation was carried out to determine the quality of the information derived from the image data (Congalton \& Green, 1999). Quantitative validation was conducted, meaning that the classified AISA data (representing the groundreference image and assumed to be $100 \%$ correct) was used to measure the simulated EnMAP classification error. The validation of the simulated EnMAP classification was conducted using a confusion matrix comparing the classified EnMAP simulation data to the classified AISA data. The 30-m spatially resampled AISA classification map was used as the ground-reference image. Table 4.10 shows the confusion matrix generated for the classification. The spatial distribution of the simulated EnMAP soil erosion state map errors can be seen in Figure 4.26. 
Table 4.10: Confusion matrix generated for the EnMAP final soil erosion state map.

\begin{tabular}{|c|c|c|c|c|}
\hline Class & $\begin{array}{l}\text { AISA Erosion } \\
\text { (Pixels) }\end{array}$ & $\begin{array}{c}\text { AISA } \\
\text { Intermediate } \\
\text { (Pixels) }\end{array}$ & $\begin{array}{c}\text { AISA } \\
\text { Accumulation } \\
\text { (Pixels) }\end{array}$ & Total (Pixels) \\
\hline $\begin{array}{l}\text { EnMAP } \\
\text { Erosion }\end{array}$ & 6 & 9 & 0 & 15 \\
\hline $\begin{array}{l}\text { EnMAP } \\
\text { Intermediate }\end{array}$ & 6 & 48 & 6 & 60 \\
\hline $\begin{array}{l}\text { EnMAP } \\
\text { Accumulation }\end{array}$ & 0 & 6 & 10 & 16 \\
\hline Total & 12 & 63 & 16 & 91 \\
\hline Class & $\begin{array}{c}\text { AISA Erosion } \\
(\%)\end{array}$ & $\begin{array}{c}\text { AISA } \\
\text { Intermediate } \\
(\%) \\
\end{array}$ & $\begin{array}{c}\text { AISA } \\
\text { Accumulation } \\
(\%)\end{array}$ & Total (\%) \\
\hline $\begin{array}{l}\text { EnMAP } \\
\text { Erosion }\end{array}$ & 50.0 & 14.3 & 0 & 16.5 \\
\hline $\begin{array}{l}\text { EnMAP } \\
\text { Intermediate }\end{array}$ & 50.0 & 76.2 & 37.5 & 65.9 \\
\hline $\begin{array}{l}\text { EnMAP } \\
\text { Accumulation }\end{array}$ & 0 & 9.5 & 62.5 & 17.6 \\
\hline Total & 100.0 & 100.0 & 100.0 & 100.0 \\
\hline Class & $\begin{array}{c}\text { Commission } \\
(\%)\end{array}$ & Omission (\%) & $\begin{array}{l}\text { Commission } \\
\text { (Pixel) }\end{array}$ & $\begin{array}{l}\text { Omission } \\
\text { (Pixel) }\end{array}$ \\
\hline $\begin{array}{l}\text { EnMAP } \\
\text { Erosion }\end{array}$ & 60.0 & 50.0 & $9 / 15$ & $6 / 12$ \\
\hline $\begin{array}{l}\text { EnMAP } \\
\text { Intermediate }\end{array}$ & 20.0 & 23.8 & $12 / 60$ & $15 / 63$ \\
\hline $\begin{array}{l}\text { EnMAP } \\
\text { Accumulation }\end{array}$ & 37.5 & 37.5 & $6 / 16$ & $6 / 16$ \\
\hline Class & $\begin{array}{c}\text { Producers } \\
\text { Accuracy (\%) }\end{array}$ & $\begin{array}{c}\text { Users } \\
\text { Accuracy (\%) }\end{array}$ & $\begin{array}{c}\text { Producers } \\
\text { Accuracy (Pixel) }\end{array}$ & $\begin{array}{c}\text { Users } \\
\text { Accuracy } \\
\text { (Pixel) }\end{array}$ \\
\hline $\begin{array}{l}\text { EnMAP } \\
\text { Erosion }\end{array}$ & 50.0 & 40.0 & $6 / 12$ & $6 / 15$ \\
\hline $\begin{array}{l}\text { EnMAP } \\
\text { Intermediate }\end{array}$ & 76.2 & 80.0 & $48 / 63$ & $48 / 60$ \\
\hline $\begin{array}{l}\text { EnMAP } \\
\text { Accumulation }\end{array}$ & 62.5 & 62.5 & $10 / 16$ & $10 / 16$ \\
\hline
\end{tabular}




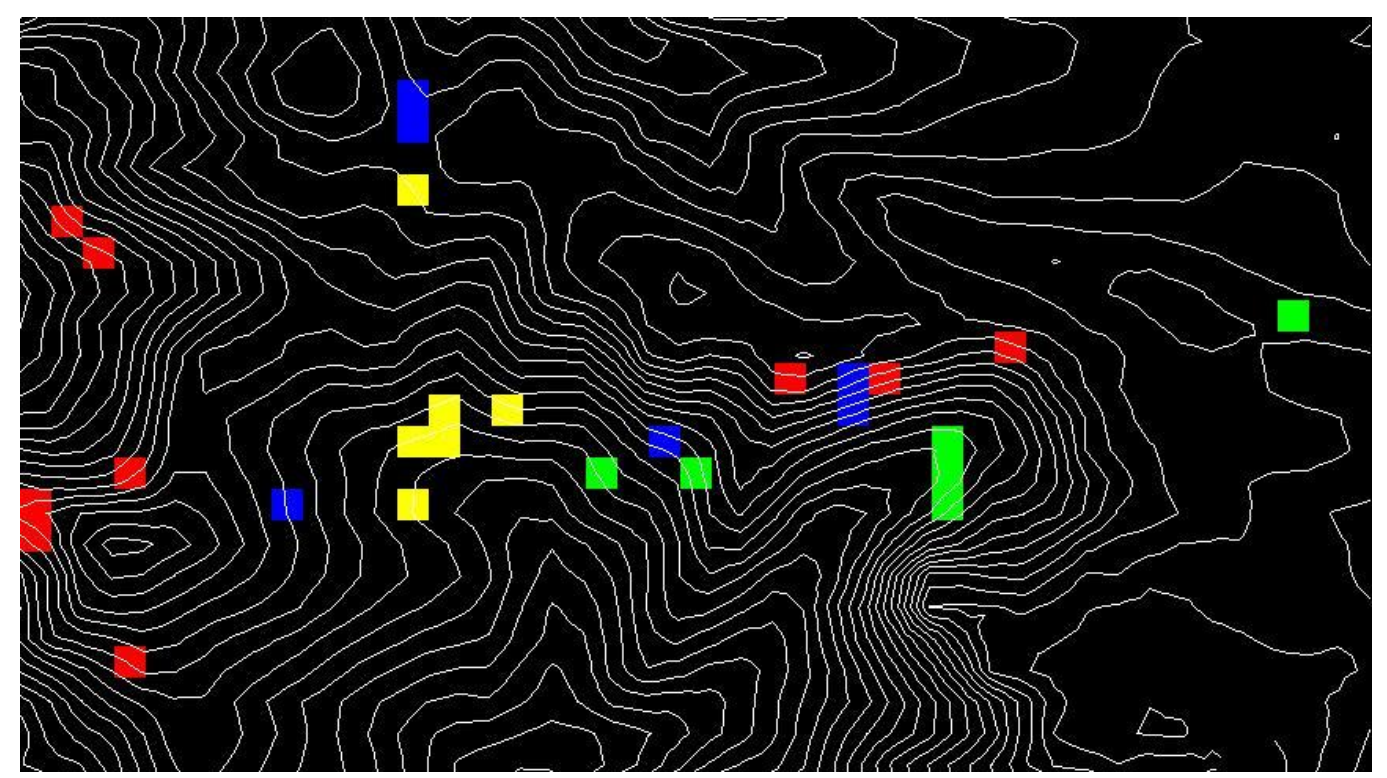

Figure 4.26: Map of the spatial distribution of classification errors overlaid by contour lines. Red = Intermediate classified as Eroded; Green = Accumulation classified as Intermediate; Blue $=$ Eroded classified as intermediate; Yellow $=$ Intermediate classified as Accumulated.

Table 4.10 indicates that there was no confusion between the eroded and accumulated classes. This is apparent because no eroded or accumulated ground reference pixels were classified in the other class. Unfortunately, this is not the case between the eroded and the intermediate class or the accumulated and the intermediate class. $50 \%$ of eroded pixels were classified as intermediate and 37 $\%$ of accumulated pixels were classified as intermediate. $76.2 \%$ of intermediate pixels were accurately classified as intermediate. A greater number of 
intermediate pixels were classified as eroded than as accumulated. The larger portion of eroded pixels classified as intermediate and the larger portion of intermediate pixels classified as eroded indicates that there is greater similarity between the eroded and intermediate class than compared to the accumulated and intermediate class. The excessive confusion with the intermediate class is believed to be due to its much larger class range than the other of the two classes. These results are consistent with the physical characteristics of the scene and the soil, where there exists a greater chemical and physical difference between the eroded and accumulated soil erosion states than between either the eroded and intermediate or accumulated and intermediate. These chemical and physical characteristics include the calcite content and clay content. The calcite content influences the reflectance values of the soil, with brighter reflectance values being consistent with eroded soils states and the clay content influences the clay absorption feature characteristic and has a stronger presence in the accumulated soils. The intermediate soil state exhibits physical and chemical characteristics of both. The highest producers and users accuracy belongs to the intermediate class at $76.2 \%$ and $80 \%$, respectively. The high producers accuracy, which is an evaluation of the ability to map the intermediate soil class, is the result of the large area of the map that is occupied by the intermediate class (Congalton and Green, 1999). 
The overall accuracy calculated for the classification is $70.3 \%$. It is calculated as the number of pixels classified correctly over the total number of pixels. In this instance 64 pixels out of a total 91 pixels were classified correctly. The kappa coefficient calculated for the classification is 0.40 . It is another measure of accuracy (agreement) used specifically for categorical data. 


\section{Discussion}

Spain is increasingly susceptible to soil erosion resulting from the long occurring changes in land use and land cover (Symeonakis et al., 2007). Remote sensing has the ability to provide timely data to assist in the management of this issue. Hyperspectral remote sensing, due to the narrow, contiguous spectral bands, has proven to be a valuable tool for identifying the very narrow absorption features characteristics of soils and, in turn, the mapping and monitoring soil erosion processes (Ben-Dor et al., 2009; Shrestha et al., 2005). This thesis research has investigated the utility of simulated EnMAP data for monitoring soil condition. This chapter discusses the importance of these results. A brief discussion of the findings presented on the pre-processing of the combined AISA Eagle and Hawk data will be followed by an in-depth discussion of the image processing results and the final accuracy assessment.

\subsection{Hyperspectral Image Pre-processing}

Standard pre-processing procedures were used to correct for common image errors and distortions (e.g., striping and geometric correction). Many errors and uncertainties remained following the pre-processing of the AISA Eagle and Hawk data used for the EeteS simulation, which was a concern as these errors may cause issues in the resultant simulated EnMAP data cube. While difficult to determine the magnitude of the various image error sources to the simulated data, the following errors were common to all images used in this 
research: the across-track gradient effects present within each flight line, the North/South topographical influences and the BRDF between the flight lines, the geometric accuracy in relation to the field data, the loss of information due to the jump correction, and the spectral miscalibration.

The cross-track illumination correction was applied to the data to remove both the across-track gradient and along-track topographical effects in the image. The results demonstrated that the image gradient effects were reduced using the cross-track illumination correction and, while other commonly applied techniques for this correction were evaluated, such as the nadir-normalization offered in ATCOR, they demonstrated no significant improvement and were not considered further. The cross-track illumination correction is similar to the nadir normalization in ATCOR but, instead of normalizing the across-track values to nadir, they are normalized based on the along-track mean values. Both correction methods were also designed for different FOVs. The nadir normalization was designed for a much wider FOV (20 degrees), whereas the cross-track illumination correction was designed for smaller (typically satellite) FOVs.

The images used for this study were impacted by significant BRDF effects. These anisotropic reflectance effects produced visible differences between all flight lines used. The available BRDF corrections offered in ATCOR were not able to compensate for these reflectance differences. It was limited because it applies a generalized correction for all surface covers when in fact, the best results are 
generated when using a per-surface cover BRDF correction (Richter and Schläpfer, 2012; Beisl, 2001; Kennedy et al., 1997). Since the SU study site straddles the seam line between two flight lines, the across-track reflectance gradient and the between flight-line BRDF effects have an influence on the reflectance data in the study site. The two flight lines will be referred to as flight line A (Easterly portion of the SU study site) and flight line B (Westerly portion of the SU study site) (Figure 3.12).

Flight line A accounts for approximately $75 \%$ of the scene with the furthest west portion of the scene ( $25 \%)$ composed of flight line B. Figure 5.1 compares spectra from a homogenous land cover that extends over the seam line. As it can be seen in Figure 5.1, flight line A contains more noise in the SWIR spectral region. The difference in reflectance value and noise content between flight lines A and B complicated the spectral unmixing within the SU site. The topographical effects in the along-track direction were not as influential because of the small North/South extent the SU study site covered and the slower rate of change in brightness compared to the across-track gradient and the intra-flight line BRDF. 


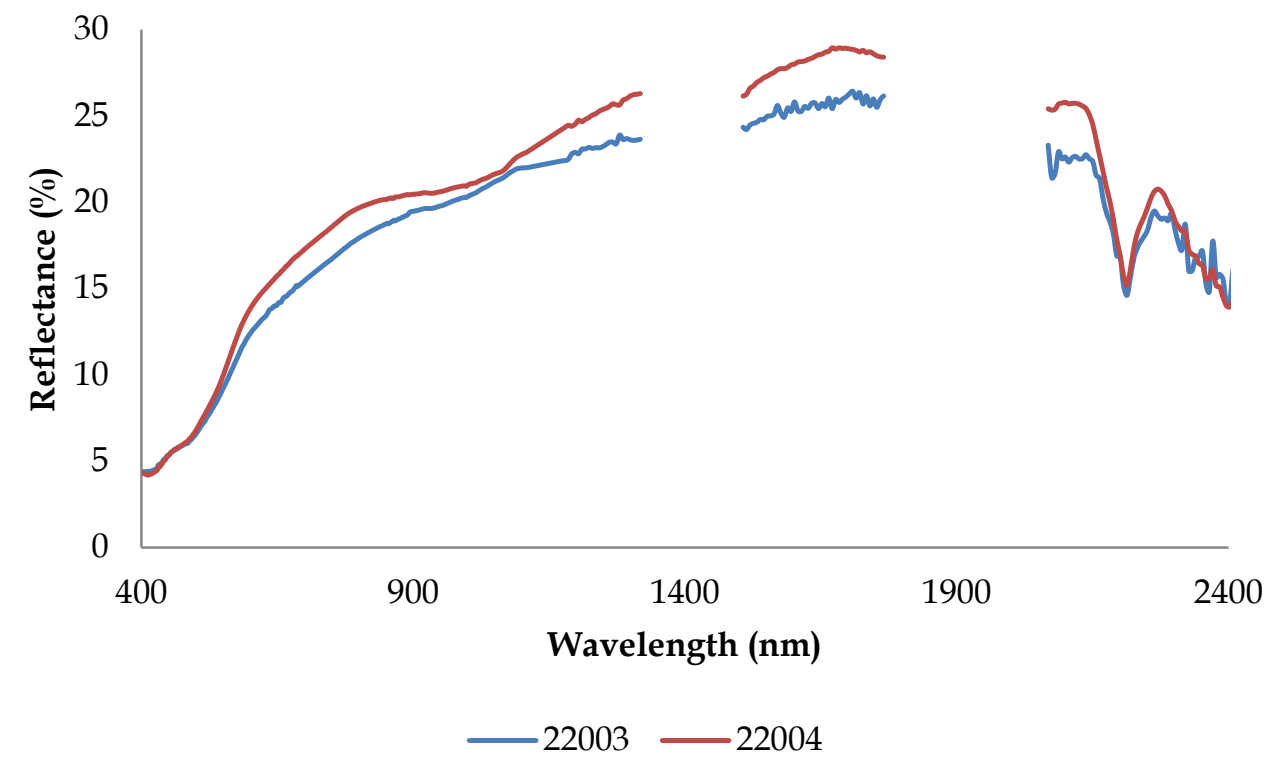

Figure 5.1: Comparison of spectra from homogenous land cover that extends over the seam line. Flight line 22003 is referred to as flight line A in the text, while flight line 22004 is referred to as flight line B.

The final RMS error resulting from the geometric correction was $5.96 \mathrm{~m}$, which is within one image pixel. Although an RMS error of one pixel is acceptable for a 6-m GSD (Palubinskas et al., 2003; Schläpfer and Richter, 2002), the scale at which the field data was collected does not allow for a one pixel offset in the image data. Figure 5.2 shows the area covered by the field plots used for the 2011 field data collection and the actual area required to account for a spatial offset of one pixel. 


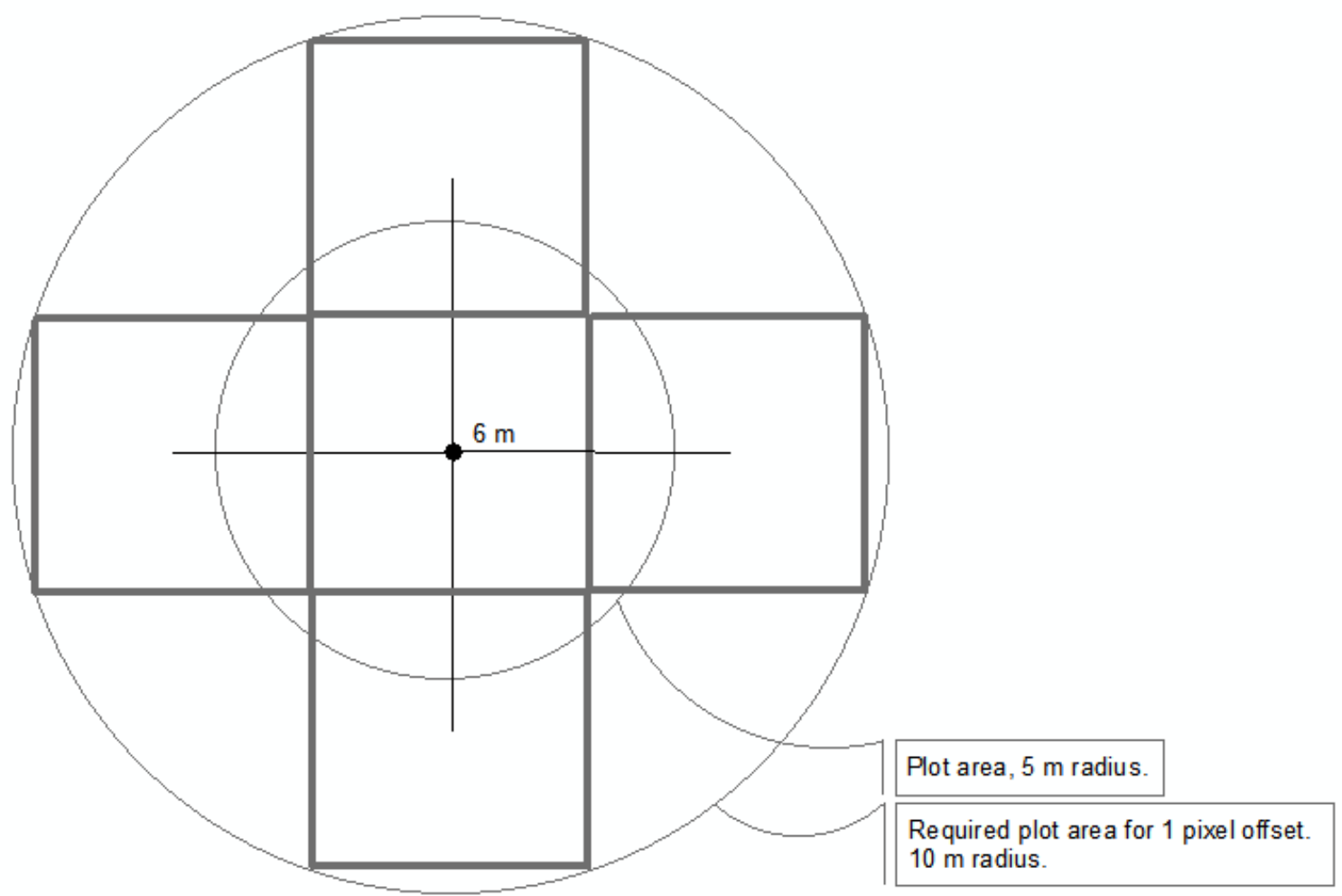

Figure 5.2: Required field plot area to allow for a 1 pixel $(6 \mathrm{~m})$ offset if employing the field sampling method utilized in this thesis. The inner circle represents the $79 \mathrm{~m}^{2}$ plot area used for the 2011 Camarena field work. The outer circle shows the plot area $\left(295 \mathrm{~m}^{2}\right)$ required to allow for a 1 pixel error in the geometric correction.

A potential implication of this geometric discrepancy on the final results concerns the accurate positioning of the fields in the AISA image data. The image endmember spectra for the soils unmixing were collected from the SU2 (eroded) and SU4 (accumulated) locations within the image. With the field plot size used 
in the 2011 Camarena field campaign, any offset in the AISA image data could result in an error locating the fields within the image, and ultimately, a spectral misrepresentation of the eroded and accumulated endmembers. Another limitation of the field plot size used in the 2011 Camarena field campaign is, due to the difference in spatial scale between the field plots and the simulated EnMAP data. The area covered by the simulated EnMAP pixel is far larger than that covered by the field data, meaning the field data would not accurately represent the surface cover contained within the 30-m simulated EnMAP pixel.

The main implication of the jump correction on the research was the loss of the iron oxide absorption feature in the $750 \mathrm{~nm}-1040 \mathrm{~nm}$ range (Chabrillat et al., 2011). This is because the majority of the data in that region was replaced to remove the spectral jump artifact from the data. This feature could have potentially assisted in more accurately identifying the soil erosion states, because there was a difference in iron content between the eroded and accumulated soil erosion states. While this error is present in this particular dataset do to the sudden shift from the VNIR to the SWIR sensor, the future EnMAP sensor will provide an overlap between the VNIR and SWIR sensors, allowing for more detailed information to be derived from this spectral region.

A spectral miscalibration, in the form of a shift (Figure 4.11), was identified in the combined AISA Eagle and Hawk data. Past studies have indicated that careful spectral calibration of hyperspectral data is required for 
high degrees of spectral accuracy (Cairns et al., 2003). Given the complications of processing the combined AISA Eagle and Hawk data, a degree of uncertainty related to the accuracy of the spectral calibration remains. Had the error been identified earlier, the spectral calibration available in ATCOR could have been applied to identify and correct for any spectral miscalibrations (Richter and Schläpfer, 2012).

As a result of the spectral miscalibration, errors (spectral spikes) were introduced into the spectral signature following the atmospheric correction. The spikes in the spectral signatures are the main reason the ELC was applied to the data. The spectral miscalibration in the data would not have only affected the atmospheric correction in the pre-processing of the AISA data, but would also have influenced the atmospheric modules in the EeteS simulation and, subsequently, the soil classification.

\subsection{Image Processing}

The goal of the image processing was to segregate the predominantly soil pixels and, subsequently, determine their soil erosion state using a combination of SMA and terrain analysis. This procedure is possible due to the strong relationship between soil colour and terrain attributes and the soils physical properties.

There was a lack of ground reference data available at the EnMAP spatial scale to validate the simulated EnMAP soil erosion state map. As a result, the 
image processing was also conducted on the AISA image so that the resulting AISA soil erosion state classification map could be used as validation for the simulated EnMAP soil erosion state map. Both the AISA and the simulated EnMAP image processing will be discussed. The three main processing steps conducted on the two data sets will be reviewed as follows: 1) the masking of green and dry vegetation, 2) the classification of soil erosion states and 3) the validation.

In the final AISA soil erosion state classification map, $18 \%$ of the scene was classified as accumulated, $13 \%$ was classified as eroded and $69 \%$ was classified as intermediate. For the final simulated EnMAP soil erosion state classification map, $18 \%$ of the scene was classified as accumulated, $16 \%$ was classified as eroded and $66 \%$ was classified as intermediate. The accumulated class was equally represented in both the AISA and the simulated EnMAP image classifications. The simulated EnMAP soil erosion state map had more pixels classified as eroded and, accordingly, fewer pixels classified as intermediate, than the AISA soil erosion state map.

The validation of the final simulated EnMAP soil erosion state map was conducted against the final 30-m one derived from the AISA data using a confusion matrix. The overall accuracy calculated for the classification was 70.3 $\%$. Confusion occurred between the intermediate class and the two extreme classes (eroded and accumulated). A total of $50 \%$ of eroded pixels were classified 
as intermediate and $37 \%$ of accumulated pixels were classified as intermediate. The highest producers and users accuracy belongs to the intermediate class at $76.2 \%$ and $80 \%$, respectively.

\subsubsection{Soil Unmixing}

The soil unmixing consisted of selecting appropriate endmembers and then unmixing the image data using MESMA. The endmembers selected for the procedure were eroded and accumulated. The objective of the soil unmixing was to quantify the contribution of both endmembers within each pixel in the image.

Spectral brightness is the primary influence on spectral differences between soil erosion stages with spectral curve shape difference being secondary (Schmid et al., 2012; Huete and Escadafal, 1991). Spectral ambiguity and endmember similarity (correlation) contributed to the non-real fraction values, resulting from the soils unmixing because of the similar brightness ranges covered by the both the eroded and accumulated endmembers as shown in Figures 3.21 and 3.22 (van der Meer and De Jong, 2000; Gross and Schott, 1998). Nevertheless, based on the results, the endmembers selected for the soils unmixing were an acceptable compromise between providing meaningful results and maintaining satisfactory RMS error values.

Three criteria were used to evaluate the validity of the unmixing results as follows: 1) the fraction values across the image are mostly between one and zero; 
2) the average RMS error is low; and 3) the RMS error image shows a low spatial correlation or pattern in the error present (van der Meer, 1995).

Non-real fraction values accounted for approximately $3 \%$ of the AISA image and $12 \%$ of the EnMAP image (Tables 4.5 and 4.8). Ideally, non-real fraction values would not be present within the fraction images. This would have been possible had a fully-constrained unmixing procedure been employed. However, MESMA does not place constraints on the fraction values, meaning values below zero and above one will occur.

Linear spectral mixture analysis makes a series of essential assumptions including: 1) the landscape is composed of a few fundamental components, which are spectrally distinct, 2) the spectral signature of the component is a constant within the entire spatial extent of the scene, and 3) the remotely sensed signal of a pixel is linearly related to the fractions of the ground components (Song, 2005). Each of these assumptions would contribute to the presence of non-real fraction values within an unconstrained linear unmixing unless all endmembers are accounted for. Endmember variability (assumption \#2) is a fundamental component of MESMA so this can be excluded from the discussion. However, assumptions number one and number three are contributors to the non-real fraction values. With the majority of both the AISA and the simulated EnMAP fraction images falling between zero and one, the results from the unmixing are not uncommon. 
The AISA unmixing was additionally evaluated against the known field characteristics of the four remaining sampling sites within the SU site (SU1, SU3, SU5 and SU6). The soil erosion states within the SU study site were defined based on physical and chemical properties of the soil, primarily calcite. Its increasing presence is indicative of a state of erosion (Schmid et al., 2012).This

Table 5.1: The eroded fraction values from each field location and the calcite content measured from soils samples collected from the field sites.

\begin{tabular}{|c|c|c|c|}
\hline $\begin{array}{c}\text { Field } \\
\text { Location } \\
\text { Identifier }\end{array}$ & Erosion State & $\begin{array}{c}\text { Eroded } \\
\text { Fraction } \\
\text { Value }\end{array}$ & $\begin{array}{c}\text { Calcite Content } \\
\text { (semiquantitative \%) }\end{array}$ \\
\hline SU2 & Most Advanced Erosion & 0 & 42 \\
\hline SU6 & Advanced Erosion & 0.071 & 40 \\
\hline SU5 & Advanced Erosion & 0.29 & 7 \\
\hline SU1 & Middle & 0.52 & 5 \\
\hline SU3 & Middle & 0.79 & 0 \\
\hline SU4 & Accumulation & 1 & 0 \\
\hline
\end{tabular}

relationship was also seen in the remote sensing data, which provide evidence that hyperspectral data are able to detect differences in calcite concentrations. This is in accordance with existing literature, such as the detection of calcium carbonate using HYMAP in the La Peyne Valley area in southern France (Lagacherie et al., 2008). An $\mathrm{r}^{2}$ value of 0.76 between the eroded fraction value and the measured calcite content from each sampling location was identified. Table 5.1 presents the eroded fraction values against the measured calcite content. 


\subsubsection{Class Definition}

The soil erosion classes selected for this research were eroded, accumulated and intermediate. The soil erosion classes used were based on the soil erosion states identified in the SU site field data. Eroded and accumulated image endmembers were collected from the SU2 and SU4 field locations. The unmixing resulted in fraction maps for both of the endmembers. The soil erosion stages were generated based on the within pixel mixture of eroded and accumulated endmembers.

Exploiting known relationships between topography and landscape elements through pre-classification can improve the results of image interpretation (Florinsky, 1998) and classification (Franklin, 1990; Hutchinson, 1982; Richards et al., 1982).The relationship between soil properties and terrain characteristics is well documented and often applied in soil feature prediction and soil landscape modelling (Dobos et al., 2000; Bell et al., 1994; Moore et al., 1993).

Using a pre-categorization for defining the soil erosion classes was the best possible solution for this research, because classes could not be derived directly from the fraction maps and topography is a dominant factor contributing to soil properties in agricultural regions. 


\subsubsection{Accuracy Assessment}

The accuracy of a classification is measured by the degree to which the derived image classification agrees with reality or the "truth" (Campbell, 1996; Janssen and van der Wel, 1994). The accuracy assessment for the EnMAP soil erosion state map was evaluated using a confusion matrix.

The accuracy of the soil erosion state map was influenced by the compounding errors from the pre-processing of the AISA data (Lunetta et al., 1991). Compounding errors such as that introduced due to sensor miscalibration (spectral shift and across-track gradient), BRDF, and scaling issues between the field data and the airborne data, are all believed to have had an impact on the final data product (Canters, 1997; Czaplewski, 1992). Without adequate understanding and quantification of the errors introduced by these sources, their effect cannot be appropriately compensated for in the final classification accuracy assessment. Furthermore, additional external error would have been introduced into the final accuracy assessment of the simulated EnMAP soil erosion state map, because it was conducted against a remotely sensed data product, the $30-\mathrm{m}$ AISA derived soil erosion state map (Foody, 2002).

The overall accuracy of the classification was $70.3 \%$ with a kappa coefficient of 0.40. Complete agreement between the simulated EnMAP classification and the AISA reference data would result in a Kappa coefficient of one. An overall accuracy of $85 \%$ with no individual class falling below $70 \%$ has 
been defined by Tomlinson et al. (1999) as the minimum requirement for classification accuracy. However, in the evaluation of 25 papers published between 1994 and 1995, Trodd (1995) found that the accuracies obtained in the papers generally fell below the recommended $85 \%$. 


\section{Conclusions}

This study investigated the potential of the future EnMAP satellite in the detection and mapping of soil erosion features in semi-arid Mediterranean environments. Monitoring of soil erosion is essential due to our dependence on this resource for the production of food and fiber, as well as for its required role in many atmospheric, hydrospheric and lithospheric functions. Due to the relative importance of the soils as a medium for plant growth, a recycling system for organic materials, and a storage system for the supply and purification of water, monitoring is critical to maintaining this vital natural resource. The monitoring of soil erosion is especially necessary in areas with a high susceptibility to soil erosion, such as semi-arid Mediterranean environments.

A simulated EnMAP hyperspectral data cube was generated from airborne hyperspectral VNIR and SWIR image data for the purpose of this research. Detection and mapping of soil erosion features was achieved using spectral unmixing techniques (MESMA) and terrain derivatives for defining erosion class boundaries. The information products produced in this study include eroded and accumulated fraction maps and a final simulated EnMAP soil erosion state classification map portraying eroded, intermediate and accumulated soil erosion state classes.

The overall classification accuracy of the soil erosion state map generated using the simulated EnMAP data was $70.3 \%$. The simulated EnMAP data were 
able to successfully discriminate between soil and vegetation cover. They were also able to identify variations in calcite content which is a key identifier of soil erosion in semi-arid Mediterranean environments. Although the simulated EnMAP soil erosion state map did not meet the minimum accuracy requirement of $85 \%$ as defined by Thomlinson et al. (1999), it can be concluded that the future EnMAP satellite will be able to map soil erosion states in these environments.

Mapping involves the presentation of information within a spatial context. With this in mind, the evaluation of the research hypothesis and the research objectives consisted of the following questions:

1) Can a simulated EnMAP hyperspectral image provide information on soil erosion states, and

2) Can a simulated EnMAP hyperspectral image effectively represent them spatially?

EnMAP's ability to spatially represent the soil erosion stages was successful because the broad class definitions (eroded, intermediate and accumulated) selected for the mapping could be detected effectively at a spatial scale that was compatible with the 30-m GSD of the image data.

The confusion between soil erosion classes indicated that there is a potential limitation in the future EnMAP sensors ability to detect key spectral features related to soil erosion. This is largely related to high levels of noise in the 
SWIR region between $2000 \mathrm{~nm}$ and $2500 \mathrm{~nm}$ of the simulated image spectra, a region which is heavily relied on for mineral characterization. However, the noise in this region may have been a remnant artifact from pre-processing measures and the original AISA data itself, and is not a sure indicator of potential limitations of the EnMAP sensor.

High-spectral resolution and a high SNR in the SWIR region are required for soil erosion detection (Chabrillat et al., 2013; Escribano et al., 2010). EnMAP will provide coverage of the $400 \mathrm{~nm}$ to $2450 \mathrm{~nm}$ range with approximately 240 bands and a peak SNR of 170:1 in the $2000 \mathrm{~nm}$ to $2500 \mathrm{~nm}$ region. Current satellite sensors such as Hyperion, which provides a peak SNR of 50:1 in this region, and the Compact High Resolution Imaging Spectrometer (CHRIS) which only provides information in the VNIR, do not have sensor characteristics appropriate for many soil investigations. EnMAP will be able to overcome these limitations by providing both high-spectral resolution data and a high SNR in the SWIR region.

The conclusions formulated from this research were stated solely in the scope of the spatial scale to which the soil erosion process under investigation occurred and at the level of detail that the information had been extracted (Lam et al., 1992; Woodcock and Strahler, 1987). The conclusions derived from this research would not be applicable to investigations where the level of information being derived is at a much higher level of detail, such as narrower class 
definitions, or if the spatial scale of the erosion process under investigation changes.

It was assumed that the high- spatial variability related to soil erosion in semi-arid Mediterranean environments (Schlesinger and Pilmanis, 1998; Puech, 1994) would not have been effectively discriminated by the moderate spatial resolution of the EnMAP sensor. However, the spectral characteristics of the sensor make inter-pixel estimations of surface cover possible (e.g., SMA, MTMF; Malenovský et al., 2007; Shrestha et al., 2005; Clark, 1999), which helps to reduce any possibly limitations related to the spatial resolution of the sensor. The fraction maps indicate that the high-spatial variability of surface soil state can effectively be captured within the EnMAP 30-m GSD using spectral decomposition techniques.

The final soil erosion stage map shows that discrete soil erosion state classes can be derived from soil surface composition fraction maps with the assistance of digital terrain data. As indicated in previous studies, such as that conducted by Dobos et al. (2000), the inclusion of terrain attributes in the form of terrain derivatives are useful for enhancing upon solely spectral classifications of these features.

The simulation of satellite data from airborne data is extremely difficult due to differences in sensor design, operation and spatial resolution. All of these factors are well known and while complex and difficult to achieve, are possible. 
Other difficulties were present as the EnMAP sensor is not yet launched and the parameters of the eventual sensor are only estimates from the design specification of the instrument. The quality of the input data into the EeteS simulation was not ideal and had direct effects on the output simulated EnMAP data. This being said, results identifying and discriminating between soil erosion stages were still obtained, demonstrating the robustness of the future EnMAP data product. Overall, this research showed that the applicability of the future EnMAP satellite sensor to soil erosion investigations in semi-arid Mediterranean environments is optimistic. Accordingly, these results confirm that EnMAP will make an important contribution to Mediterranean soil monitoring and management.

Recommendations concerning future investigations into the capabilities and potential limitations of the future EnMAP sensor regarding soil erosion mapping in semi-arid Mediterranean environments include:

1) Using simulated EnMAP data to map soil erosion in a variety of regions where the patterns of the soil erosion occur at different spatial scales, and

2) Classifying soil erosion states using simulated EnMAP data at varying levels of class complexity.

To successfully accomplish these research objectives it would be necessary to modify certain procedures introduced in this research. In particular, it would 
be beneficial to include more detailed field observations such as density cover in vegetated areas and fractional land surface cover, especially if implementing any spectral decomposition methods in the study. As illustrated in this research, the input data quality directly affects the quality and accuracy of the output simulated EnMAP data and consequently, the results. Therefore, a focus on input data quality into the simulation would also be essential. With detailed attention given to the collection of appropriate field data and to overall data quality, future investigations such as these would provide further insight into soil erosion mapping using image data as provided by the future EnMAP satellite sensor. 


\section{REFERENCES}

Adams, R. M. (1981). Heartland of cities: Surveys of ancient settlement and land use on the central floodplain of the Euphrates (pp. 88-89). Chicago: University of Chicago Press.

Adler-Golden, S. M., Matthew, M. W., Bernstein, L. S., Levine, R. Y., Berk, A., Richtsmeier, S. C., ... \& Hsiao-hua, K. B. (1999). Atmospheric correction for shortwave spectral imagery based on MODTRAN4. In SPIE's International Symposium on Optical Science, Engineering, and Instrumentation (pp. 61-69). International Society for Optics and Photonics.

Agnew, C., \& Warren, A. (1993). The sand trap. In J. Hatton \& P. Plouffe (Eds.), The Culture of Science, 517-525. New York: Macmillan.

Agriculture Canada Expert Committee on Soil Survey. (1987). The Canadian system of soil classification. (2nd ed.). Agricultural Canada Publications, 1646, p. 164.

Aidoud, A., Aidoud-Lounis, F., \& Slimani, H. (1998). Effects of grazing on soil and desertification: a review from the southern Mediterranean rim. Ecological basis of livestock grazing in Mediterranean ecosystems (Papanastasis, VP E Peter, D. Eds.). European Commission EUR, 18308, 133-148.

Aktaruzzaman, A. (2008). Simulation and Correction of Spectral Smile Effect and its Influence on Hyperspectral Mapping. Doctoral dissertation, MS Thesis, International Institute for Geo-Information Science and Earth Observation. Enschede, Netherlands.

Andréfouët, S., \& Roux, L. (1998). Characterisation of ecotones using membership degrees computed with a fuzzy classifier. International Journal of Remote Sensing, 19(16), 3205-3211.

ARSF. Data_quality-20110624. Interim Report (Hyperspectral), June 24th, 2011. Retrieved from: http://arsf-dan.nerc.ac.uk/trac/wiki/Reports.

Asner, G. P., \& Heidebrecht, K. B. (2003). Imaging spectroscopy for desertification studies: comparing AVIRIS and EO-1 Hyperion in Argentina drylands. Geoscience and Remote Sensing, IEEE Transactions on, 41(6), 1283-1296. 
Atkinson, P. M. (1997). Selecting the spatial resolution of airborne MSS imagery for small-scale agricultural mapping. International Journal of Remote Sensing, 18(9), 1903-1917.

Barnes, E. M., \& Baker, M. G. (2000). Multispectral data for mapping soil texture: possibilities and limitations. Applied Engineering in Agriculture, 16(6), 731-746.

Barnsley, M., \& Curran, P. (1990). The role of airborne remote sensing in terrestrial ecology, Remote Sensing and Global Change. Nottingham :Remote Sensing Society.

Barth, H. K. (1982). Accelerated erosion of fossil dunes in the Gourma region (Mali) as a manifestation of desertification. In Acidic soils and geomorphic processes: proceedings of the International Conference of the International Society of Soil Science, Jerusalem, Israel, March 19-April 4, 1981/DH Yaalon, ed. Cremlingen : Catena Verlag.

Bartholomeus, H., Epema, G., \& Schaepman, M. (2007). Determining iron content in Mediterranean soils in partly vegetated areas, using spectral reflectance and imaging spectroscopy. International Journal of Applied Earth Observation and Geoinformation, 9(2), 194-203.

Bateson, A., \& Curtiss, B. (1996). A method for manual endmember selection and spectral unmixing. Remote Sensing of Environment, 55(3), 229-243.

Bazzaz, F. A. (1968). Succession on abandoned fields in the Shawnee Hills, southern Illinois. Ecology, 924-936.

Beasley, D. B., Huggins, L. F., \& Monke, E. J. (1980). ANSWERS: A model for watershed planning. Transactions of the ASAE-American Society of Agricultural Engineers.

Beckett, P. H. T., \& Burrough, P. A. (1971). The relation between cost and utility in soil survey. Journal of soil science, 22(4), 466-480.

Behrens, T., \& Scholten, T. (2006). Digital soil mapping in Germany - a review. Journal of Plant Nutrition and Soil Science, 169(3), 434-443.

Beisl. (2001). Correction of bidirectional effects in imaging spectrometer data. Remote Sensing Series, Vol. 37 Remote Sensing Laboratories, University of Zürich: Zürich, Switzerland.

Beisl, U., \& Woodhouse, N. (2004, July). Correction of atmospheric and bidirectional effects in multispectral ADS40 images for mapping purposes. In Proc. XXth Congress of the ISPRS. 
Bell, J. C., Cunningham, R. L., \& Havens, M. W. (1994). Soil drainage class probability mapping using a soil-landscape model. Soil Science Society of America Journal, 58(2), 464-470.

Ben-Dor, E. (2002). Quantitative remote sensing of soil properties. Advances in Agronomy, 75, 173-243.

Ben-Dor, E., \& Banin, A. (1995). Near-infrared analysis as a rapid method to simultaneously evaluate several soil properties. Soil Science Society of America Journal, 59(2), 364-372.

Ben-Dor, E., Chabrillat, S., Demattê, J. A. M., Taylor, G. R., Hill, J., Whiting, M. L., \& Sommer, S. (2009). Using imaging spectroscopy to study soil properties. Remote Sensing of Environment, 113, S38-S55.

Ben-Dor, E., Feingersh, T., Filin, S., \& Schläpfer, D. (2010). Better analysis of hyperspectral images by correcting reflectance anisotropy. SPIE Newsroom,(Apr. 7, 2010), 3.

Ben-Dor, E., Irons, J. A., \& Epema, A. (1999). Soil Spectroscopy Manual of Remote Sensing (Third ed., pp. 111-188). New York, NY.: J. Wiley \& Sons, Inc..

Benito, G., \& Sancho, C. (1992). Erosion rates in badland areas of the central Ebro Basin (NE-Spain). Catena, 19(3), 269-286.

Bennett, H. H. (1960). Soil erosion in Spain. Geographical Review, 59-72.

Berk, A., Bernstein, L. S., Anderson, G. P., Acharya, P. K., Robertson, D. C., Chetwynd, J. H., \& Adler-Golden, S. M. (1998). MODTRAN cloud and multiple scattering upgrades with application to AVIRIS. Remote Sensing of Environment, 65(3), 367-375.

Betts, N. L., Cruickshank, M. M., \& Tomlinson, R. W. (1986). An evaluation of SPOT-simulation imagery for land-use mapping and ecological investigations in upland areas of Northern Ireland. International Journal of Remote Sensing, 7(6), 779790.

Binger, N. and Ory, T. (1984). The 1983 U.S. SPOT simulation campaign: Maximizing the Potential of Aerial Image Acquisition. Proceeding in XVth ISPRS Congress, Part A8, 1-9.

Bluesky International Limited. (N.D.). LiDAR-UK. Retrieved from : http://www.lidar-uk.com/how-lidar-works/. 
Blum, W. E., Büsing, J., \& Montanarella, L. (2004). Research needs in support of the European thematic strategy for soil protection. TRAC Trends in Analytical Chemistry, 23(10), 680-685.

Boardman, J. W., \& Kruse, F. A. (1994). Automated spectral analysis: a geological example using AVIRIS data, north Grapevine Mountains, Nevada. In Proceedings of the Thematic Conference on Geologic Remote Sensing (1), 1-407. Environmental Research Institute of Michigan.

Boardman, J. W., Kruse, F. A., \& Green, R. O. (1995). Mapping target signatures via partial unmixing of AVIRIS data. Retrieved from: http://hdl.handle.net/2014/33635.

Bodechtel, J. (2001). Requirements on optical sensors for quantitative definition of surface parameters multispectral-hyperspectral. Advances in Space Research, 28(1), 241-250.

Boellstorff, D., \& Benito, G. (2005). Impacts of set-aside policy on the risk of soil erosion in central Spain. Agriculture, ecosystems \& environment, 107(2), 231-243.

Boer, M. M., \& Puigdefabregas, J. (2003). Predicting potential vegetation index values as a reference for the assessment and monitoring of dryland condition. International Journal of Remote Sensing, 24(5), 1135-1141.

Börner, A., Wiest, L., Keller, P., Reulke, R., Richter, R., Schaepman, M., \& Schläpfer, D. (2001). SENSOR: a tool for the simulation of hyperspectral remote sensing systems. ISPRS Journal of Photogrammetry and Remote Sensing, 55(5), 299312.

Bouma, J., \& Van Lanen, H. A. J. (1987). Transfer functions and threshold values: from soil characteristics to land qualities.

Briggs, D., Giordano, A., Cornaert, M. H., Peter, D., \& Maes, J. (1992). CORINE soil erosion risk and important land resources in the southern regions of the European Community. Commission of the European Communities Publication EUR, 13233.

Brown, J. F., Loveland, T. R., Ohlen, D. O., \& Zhu, Z. L. (1999). The global landcover characteristics database: the users' perspective. Photogrammetric Engineering and Remote Sensing, 65(9), 1069-1074.

Buchan, G. M., \& Hubbard, N. K. (1986). Remote sensing in land-use planning: an application in west central Scotland using SPOT-simulation data. International Journal of Remote Sensing, 7(6), 767-777. 
Butzer, K. W. (2005). Environmental history in the Mediterranean world: crossdisciplinary investigation of cause-and-effect for degradation and soil erosion. Journal of Archaeological Science, 32(12), 1773-1800.

Cairns, B., Carlson, B. E., Ying, R., Lacis, A. A., \& Oinas, V. (2003). Atmospheric correction and its application to an analysis of Hyperion data. Geoscience and Remote Sensing, IEEE Transactions on, 41(6), 1232-1245.

Cambardella, C. A., Moorman, T. B., Parkin, T. B., Karlen, D. L., Novak, J. M., Turco, R. F., \& Konopka, A. E. (1994). Field-scale variability of soil properties in central Iowa soils. Soil Science Society of America Journal, 58(5), 1501-1511.

Campbell, J. (1996). Introduction to remote sensing (2nd ed.). London: Taylor and Francis.

Campbell, K. (1983). Statistical techniques using NURE airborne geophysical data and NURE geochemical data. Computers \& Geosciences, 9(1), 17-21.

Campbell, J. B., \& Browder, J. O. (1995). Field data collection for remote sensing analysis: SPOT data, Rondonia, Brazil. Remote Sensing, 16(2), 333-350.

Canters, F. (1997). Evaluating the Uncertainty of Area Estimates Derived from Fuzzy Land-Cover Classification. Photogrammetric Engineering \& Remote Sensing, 63(4), 403-414.

Carfantan, H., \& Idier, J. (2010). Statistical linear destriping of satellite-based pushbroom-type images. Geoscience and Remote Sensing, IEEE Transactions on, 48(4), 1860-1871.

Chabrillat, S., Ben-Dor, E., Visscarra-Rossel, R. and Dematte, J. (2013).

Quantitative Soil Spectroscopy. In Quantitative Soil Spectroscopy, 1-3. Applied and Environmental Soil Science.

Chabrillat, S., Eisele, A., Guillaso, S., Rogass, C., Ben-Dor, E., \& Kaufmann, C. (2011). HYSOMA: an easy-to-use software interface for soil mapping applications of hyperspectral imagery. Paper presented at the $31^{\text {st }}$ EARSeL Symposium. Prague: Czech Republic.

Chabrillat, S., Goetz, A. F., Krosley, L., \& Olsen, H. W. (2002). Use of hyperspectral images in the identification and mapping of expansive clay soils and the role of spatial resolution. Remote Sensing of Environment, 82(2), 431-445. 
Chakravortty, S., \& Chakrabarti, S. (2011). Preprocessing of Hyperspectral Data: A case study of Henry and Lothian Islands in Sunderban Region, West Bengal, India. International Journal of Geomatics and Geosciences, 2(2), 490-501.

Chander, G., Helder, D. L., \& Boncyk, W. C. (2002). Landsat- $4 / 5$ band 6 relative radiometry. Geoscience and Remote Sensing, IEEE Transactions on, 40(1), 206-210.

Charlton, M. E., Large, A. R., \& Fuller, I. C. (2003). Application of airborne LiDAR in river environments: the River Coquet, Northumberland, UK. Earth surface processes and landforms, 28(3), 299-306.

Chavez Jr, P. S. (1988). An improved dark-object subtraction technique for atmospheric scattering correction of multispectral data. Remote sensing of environment, 24(3), 459-479.

Chen, X. F., Chen, J. M., An, S. Q., \& Ju, W. M. (2007). Effects of topography on simulated net primary productivity at landscape scale. Journal of environmental management, 85(3), 585-596.

Chen, C. M., Hepner, G. F., \& Forster, R. R. (2003). Fusion of hyperspectral and radar data using the IHS transformation to enhance urban surface features. ISPRS Journal of photogrammetry and Remote Sensing, 58(1), 19-30.

Chen, Z. S., Hsieh, C. F., Jiang, F. Y., Hsieh, T. H., \& Sun, I. F. (1997). Relations of soil properties to topography and vegetation in a subtropical rain forest in southern Taiwan. Plant Ecology, 132(2), 229-241.

Chen, J. M., Pavlic, G., Brown, L., Cihlar, J., Leblanc, S. G., White, H. P., ... \& Pellikka, P. K. E. (2002). Derivation and validation of Canada-wide coarseresolution leaf area index maps using high-resolution satellite imagery and ground measurements. Remote sensing of environment, 80(1), 165-184.

Cheng, K. S., Yeh, H. C., \& Tsai, C. H. (2000). An anisotropic spatial modeling approach for remote sensing image rectification. Remote sensing of environment, 73(1), 46-54.

Chong, A. K., \& Pearson, C. (1998). Geometrically corrected digital images for resource mapping and precision farming. In 10th Colloquium of the Spatial Information Research Centre, 16-19.

Clark, R. N. (1999). Spectroscopy of rocks and minerals, and principles of spectroscopy. Manual of remote sensing, 3, 3-58. 
Clark, R. N., King, T. V., Klejwa, M., Swayze, G. A., \& Vergo, N. (1990). High spectral resolution reflectance spectroscopy of minerals. Journal of Geophysical Research: Solid Earth (1978-2012), 95(B8), 12653-12680.

Cloutis, E. A. (1996). Review Article Hyperspectral geological remote sensing: evaluation of analytical techniques. International Journal of Remote Sensing, 17(12), 2215-2242.

Coburn, C. A., \& Peddle, D. R. (2006). A low-cost field and laboratory goniometer system for estimating hyperspectral bidirectional reflectance. Canadian Journal of Remote Sensing, 32(3), 244-253.

Cole, N. J., \& Boettinger, J. L. (2007). Pedogenic understanding raster classification methodology for mapping soils, Powder River Basin, Wyoming, USA. Digital soil mapping: an introductory perspectives. Amsterdam: Elsevier, 377-388.

Colwell, R. N., U̦laby, F. T., Simonett, D. S., Estes, J. E., \& Thorley, G. A. (1983). Manual of remote sensing. Volume 2. Interpretation and applications. American Society of Photogrammetry.

Congalton, R. G. (1991). A review of assessing the accuracy of classifications of remotely sensed data. Remote sensing of environment, 37(1), 35-46.

Congalton, R. G., Balogh, M., Bell, C., Green, K., Milliken, J. A., \& Ottman, R. (1998). Mapping and monitoring agricultural crops and other land cover in the Lower.

Congalton, R. \& Green, K. (1999). Assessing the Accuracy of Remotely Sensed Data. CRC Press Inc.

Cook, A. E., \& Pinder, J. E. (1996). Relative accuracy of rectifications using coordinates determined from maps and the global positioning system.

Photogrammetric engineering and remote sensing, 62(1), 73-78.

Cota, S. A., Bell, J. T., Boucher, R. H., Dutton, T. E., Florio, C. J., Franz, G. A., ... \& Willkinson, T. S. (2008). PICASSO: an end-to-end image simulation tool for space and airborne imaging systems. In Optical Engineering+ Applications, 708703708703. International Society for Optics and Photonics.

Cracknell, A. \& Hayes, L. (1993). Introduction to Remote Sensing. London: Taylor and Francis. 
Curran, P. J. (1989). Remote sensing of foliar chemistry. Remote sensing of Environment, 30(3), 271-278.

Czaplewski, R. L. (1992). Misclassification bias in areal estimates. Photogrammetric Engineering and Remote Sensing, 58(2), 189-192.

Dadon, A., Ben-Dor, E., \& Karnieli, A. (2010). Use of derivative calculations and minimum noise fraction transform for detecting and correcting the spectral curvature effect (smile) in Hyperion images. Geoscience and Remote Sensing, IEEE Transactions on, 48(6), 2603-2612.

Daniels, R. B., Gilliam, J. W., Cassel, D. K., \& Nelson, L. A. (1985). Soil erosion class and landscape position in the North Carolina Piedmont. Soil Science Society of America Journal, 49(4), 991-995.

Darby, H. C. (1956). The clearing of the woodland in Europe. Man's Role in Changing the Face of the Earth, 1, 183-216.

Darvishsefat, A. A., Kellenberger, T. W., \& Itten, K. I. (2002). Application of hyperspectral data for forest stand mapping. In Symposium on Geospatial Theory, Processing and Applications. Ottawa, Ontario.

Datt, B., McVicar, T. R., Van Niel, T. G., Jupp, D. L., \& Pearlman, J. S. (2003). Preprocessing EO-1 Hyperion hyperspectral data to support the application of agricultural indexes. Geoscience and Remote Sensing, IEEE Transactions on, 41(6), 1246-1259.

Daughtry, C. S. (2001). Discriminating crop residues from soil by shortwave infrared reflectance. Agronomy Journal, 93(1), 125-131.

Daughtry, C. S. T., Hunt, E. R., Doraiswamy, P. C., \& McMurtrey, J. E. (2005). Remote sensing the spatial distribution of crop residues. Agronomy Journal, 97(3), 864-871.

Daughtry, C. S. T., Hunt Jr, E. R., \& McMurtrey III, J. E. (2004). Assessing crop residue cover using shortwave infrared reflectance. Remote Sensing of Environment, 90(1), 126-134.

De Asis, A. M., \& Omasa, K. (2007). Estimation of vegetation parameter for modeling soil erosion using linear Spectral Mixture Analysis of Landsat ETM data. ISPRS Journal of Photogrammetry and Remote Sensing, 62(4), 309-324. 
De Graaff, J., \& Eppink, L. A. A. J. (1999). Olive oil production and soil conservation in southern Spain, in relation to EU subsidy policies. Land use policy, 16(4), 259-267.

Deichmann, U., \& Eklundh, L. (1991). Global digital datasets for land degradation studies: A GIS approach. Global Environment Monitoring System, United Nations Environment Programme.

De Jong, S. M., Riezebos, H. T., \& Rickson, R. J. (1994). Imaging spectroscopy, geostatistics and soil erosion models. In Conserving soil resources: European perspectives. Selected papers from the First International Congress of the European Society for Soil Conservation. (pp. 232-245). CAB INTERNATIONAL.

Dennison, P. E., Halligan, K. Q., \& Roberts, D. A. (2004). A comparison of error metrics and constraints for multiple endmember spectral mixture analysis and spectral angle mapper. Remote Sensing of Environment, 93(3), 359-367.

Dennison, P. E., \& Roberts, D. A. (2003). Endmember selection for multiple endmember spectral mixture analysis using endmember average RMSE. Remote Sensing of Environment, 87(2), 123-135.

De Ploey, J. (1989). Soil Erosion Map of Western Europe. CATENA. Leuven, Belgium: Laboratory of Experimental Geomorphology.

Deventer van, A. P., Ward, A. D., Gowda, P. H., \& Lyon, J. G. (1997). Using Thematic Mapper data to identify contrasting soil plains and tillage practices. Photogrammetric Engineering and Remote Sensing, 63, 87-93.

Dicks, S. E., \& Lo, T. H. (1990). Evaluation of thematic map accuracy in a land-use and land-cover mapping program. Photogrammetric Engineering and Remote Sensing, 56 .

Dobos, E., Micheli, E., Baumgardner, M. F., Biehl, L., \& Helt, T. (2000). Use of combined digital elevation model and satellite radiometric data for regional soil mapping. Geoderma, 97(3), 367-391.

Doran, J. W. (2002). Soil health and global sustainability: translating science into practice. Agriculture, ecosystems \& environment, 88(2), 119-127.

Doran, J. W., \& Parkin, T. B. (1994). Defining and assessing soil quality. SSSA special publication, 35, 3-3.

Doran, J. W., \& Zeiss, M. R. (2000). Soil health and sustainability: managing the biotic component of soil quality. Applied Soil Ecology, 15(1), 3-11. 
Dregne, H., \& Boyadgiev, T. G. (1983). Provisional methodology for assessment and mapping of desertification. FAO/UNEP.

Du, Q., Raksuntorn, N., Younan, N. H., \& King, R. L. (2008). End-member extraction for hyperspectral image analysis. Applied optics, 47(28), F77-F84.

Du, Y., Teillet, P. M., \& Cihlar, J. (2002). Radiometric normalization of multitemporal high-resolution satellite images with quality control for land cover change detection. Remote Sensing of Environment, 82(1), 123-134.

Dunjo, G., Pardini, G., \& Gispert, M. (2003). Land use change effects on abandoned terraced soils in a Mediterranean catchment, NE Spain. Catena, 52(1), 23-37.

Elvidge, C. D. (1990). Visible and near infrared reflectance characteristics of dry plant materials. Remote Sensing, 11(10), 1775-1795.

Escribano, P., Palacios-Orueta, A., Oyonarte, C., \& Chabrillat, S. (2010). Spectral properties and sources of variability of ecosystem components in a Mediterranean semiarid environment. Journal of Arid Environments, 74(9), 10411051.

Essery, C. I., \& Wilcock, D. N. (1986). SPOT-simulation campaign: a preliminary land-use classification for a $200 \mathrm{~km} 2$ river catchment. International Journal of Remote Sensing, 7(6), 801-814.

Eswaran, H., Lal, R. and Reich, P.F. (2001). Land Degradation: an overview. In: Bridges, E.M., Hannam, I.D., Olderman, L.R., Penning de Vries, F.W.P., Scherr, S.J. and Sompatpanit, $\mathrm{S}$ (eds.). Responses to Land Degradation. Proc. $2^{\text {nd }}$. International Conference on Land Degradation and Desertification, Khon Kaen, Thailand. Oxford Press, New Delhi, India, 2001, pp. 20-35.

EUFAR. (2010). Transnational Access Project SEDMEDHY-Official Document.

EUFAR. (2011). LiDAR Data Quality Overview. Retrieved from http://arsfdan.nerc.ac.uk/trac/wiki/Reports.

European Commission. (2002). Summary Set-Aside. Retrieved from: http://ec.europa.eu/agriculture/eval/reports/gel/sum_en.pdf.

European Commission. (2012a). The Common Agricultural Policy. A Partnership Between Europe and Farmers. Luxembourg: Publications Office of the European Union. 
European Commission. (2012b). Milestones of the CAP. Retrieved from: http:/ / ec.europa.eu/agriculture/50-years-ofcap/files/history/milestones_of_the_cap_en.pdf.

European Commission. (2014). The 1992 Reform ("MacSharry Reform"). Retrieved from: http://ec.europa.eu/agriculture/cap-history/1992-reform/index_en.htm.

Exelis Visual Information Solutions. (2014a). Other Radiometric Correction Tools. Retrieved from http://www.exelisvis.com/docs/OtherRadiometricCorrectionTools.html.

Exelis Visual Information Solutions. (2014b). Linear Spectral Unmixing. Retrieved from http:// www.exelisvis.com/docs/LinearSpectralUnmixing.html.

Exelis Visual Information Solutions. (2014c). Image Registration Tutorial. Retrieved from http://www.exelisvis.fr/docs/imageregtutorial.html.

FAO. (1967). Aerial photo interpretation in soil survey. Soils bulletin. Retrieved from http://www.fao.org/docrep/017/aq383e/aq383e.pdf.

Farrand, W. H., Singer, R. B., \& Merényi, E. (1994). Retrieval of apparent surface reflectance from AVIRIS data: A comparison of empirical line, radiative transfer, and spectral mixture methods. Remote Sensing of Environment, 47(3), 311-321.

Favis-Mortlock, D. (2007). Soil Erosion Site. Retrieved from: http://www.soilerosion.net/.

Feingersh, T., Ben-Dor, E., \& Filin, S. (2007). Improving anisotropy correction for airborne imaging spectrometers, using a LiDAR DSM and angular spectral measurements. In Proceeding of the 5 th EARSeL Workshop on Imaging Spectroscopy.

Feingersh, T., Ben-Dor, E., \& Filin, S. (2010). Correction of reflectance anisotropy: a multi-sensor approach. International Journal of Remote Sensing, 31(1), 49-74.

Florinsky, I. V. (2000). Relationships between topographically expressed zones of flow accumulation and sites of fault intersection: analysis by means of digital terrain modelling. Environmental Modelling \& Software, 15(1), 87-100.

Florinsky, I. V. (1998). Combined analysis of digital terrain models and remotely sensed data in landscape investigations. Progress in Physical Geography, 22(1), 3360. 
Florinsky, I. V., Eilers, R. G., Manning, G. R., \& Fuller, L. G. (2002). Prediction of soil properties by digital terrain modelling. Environmental Modelling \& Software, 17(3), 295-311.

Foody, G. M. (2002). Status of land cover classification accuracy assessment. Remote sensing of environment, 80(1), 185-201.

Foody, G. M., Palubinskas, G., Lucas, R. M., Curran, P. J., \& Honzak, M. (1996). Identifying terrestrial carbon sinks: classification of successional stages in regenerating tropical forest from Landsat TM data. Remote Sensing of Environment, 55(3), 205-216.

Forlani, G. \& Nardinocchi, C. (2007). Adaptive filtering of aerial laser scanning data. International Archives of Photogrammetry, Remote Sensing and Spatial Information Sciences, 36(3/W52), 130-35.

Francis, C. F., \& Thornes, J. B. (1990). Runoff hydrographs from three Mediterranean vegetation cover types. Vegetation and erosion. Processes and environments., 363-384.

Franke, J., Roberts, D. A., Halligan, K., \& Menz, G. (2009). Hierarchical multiple endmember spectral mixture analysis (MESMA) of hyperspectral imagery for urban environments. Remote Sensing of Environment, 113(8), 1712-1723.

Franklin, S. E. (1987). Geomorphometric processing of digital elevation models. Computers \& Geosciences, 13(6), 603-609.

Franklin, S. E. (1990). Topographic context of satellite spectral response. Computers E Geosciences, 16(7), 1003-1010.

García-Ruiz, J. M. (2010). The effects of land uses on soil erosion in Spain: a review. Catena, 81(1), 1-11.

García-Ruiz, J. M., Lasanta, T., Ortigosa, L., Ruiz-Flaño, P., Martí, C., \& González, C. (1995). Sediment yield under different land uses in the Spanish Pyrenees. Mountain Research and Development, 229-240.

Gessler, P. E. (1996). Statistical soil-landscape modelling for environmental management (Doctoral dissertation, Australian National University).

Gessler, P. E., Moore, I. D., McKenzie, N. J., \& Ryan, P. J. (1995). Soil-landscape modelling and spatial prediction of soil attributes. International Journal of Geographical Information Systems, 9(4), 421-432. 
Gill, T. K., \& Phinn, S. R. (2009). Improvements to ASTER-derived fractional estimates of bare ground in a savanna rangeland. Geoscience and Remote Sensing, IEEE Transactions on, 47(2), 662-670.

Gilland, B. (2002). World population and food supply: Can food production keep pace with population growth in the next half-century?. Food Policy, 27(1), 47-63.

Goetz, A. F., Vane, G., Solomon, J. E., \& Rock, B. N. (1985). Imaging spectrometry for earth remote sensing. Science, 228(4704), 1147-1153.

Goldman, D. B., \& Chen, J. H. (2005, October). Vignette and exposure calibration and compensation. In Computer Vision, 2005. ICCV 2005. Tenth IEEE International Conference, (1), 899-906. IEEE.

Goodenough, D. G., Dyk, A., Niemann, K. O., Pearlman, J. S., Chen, H., Han, T., ... \& West, C. (2003). Processing Hyperion and ALI for forest classification. Geoscience and Remote Sensing, IEEE Transactions on, 41(6), 1321-1331.

Gotway, C. A., Ferguson, R. B., Hergert, G. W., \& Peterson, T. A. (1996). Comparison of kriging and inverse-distance methods for mapping soil parameters. Soil Science Society of America Journal, 60(4), 1237-1247.

Govers, G., Vandaele, K., Desmet, P., Poesen, J., \& Bunte, K. (1994). The role of tillage in soil redistribution on hillslopes. European Journal of Soil Science, 45(4), 469-478.

Gray, D. H., \& Leiser, A. T. (1982). Biotechnical slope protection and erosion control. Van Nostrand Reinhold Company Inc..

Green, R. O. (1998). Spectral calibration requirement for Earth-looking imaging spectrometers in the solar-reflected spectrum. Applied Optics, 37(4), 683-690.

Green, A. A., Berman, M., Switzer, P., \& Craig, M. D. (1988). A transformation for ordering multispectral data in terms of image quality with implications for noise removal. Geoscience and Remote Sensing, IEEE Transactions on, 26(1), 65-74.

Griffin, M. K., \& Burke, H. H. K. (2003). Compensation of hyperspectral data for atmospheric effects. Lincoln Laboratory Journal, 14(1), 29-54.

Gross, H. N., \& Schott, J. R. (1998). Application of spectral mixture analysis and image fusion techniques for image sharpening. Remote Sensing of Environment, 63(2), 85-94. 
Grove, A. T., \& Rackham, O. (2003). The nature of Mediterranean Europe: an ecological history. Yale University Press.

Gruninger, J. H., Ratkowski, A. J., \& Hoke, M. L. (2004). The sequential maximum angle convex cone (SMACC) endmember model. In Defense and Security, 1-14. International Society for Optics and Photonics.

Grunwald, S. (2010). Current state of digital soil mapping and what is next. In Digital Soil Mapping (pp. 3-12). Springer Netherlands.

Guanter, L., Estellés, V., \& Moreno, J. (2007). Spectral calibration and atmospheric correction of ultra-fine spectral and spatial resolution remote sensing data. Application to CASI-1500 data. Remote Sensing of Environment, 109(1), 54-65.

Guanter, L., Segl, K., \& Kaufmann, H. (2009). Simulation of optical remotesensing scenes with application to the enmap hyperspectral mission. Geoscience and Remote Sensing, IEEE Transactions on, 47(7), 2340-2351.

Haboudane, D., Bonn, F., Royer, A., Sommer, S., \& Mehl, W. (2002). Land degradation and erosion risk mapping by fusion of spectrally-based information and digital geomorphometric attributes. International Journal of remote sensing, 23(18), 3795-3820.

Hajj el, M., Bégué, A., Lafrance, B., Hagolle, O., Dedieu, G., \& Rumeau, M. (2008). Relative radiometric normalization and atmospheric correction of a SPOT 5 time series. Sensors, 8(4), 2774-2791.

Helming, K., Römkens, M. J. M., \& Prasad, S. (1998). Surface roughness related processes of runoff and soil loss: a flume study. Soil Science Society of America Journal, 62(1), 243-250.

Herrick, J. E. (2000). Soil quality: an indicator of sustainable land management?. Applied Soil Ecology, 15(1), 75-83.

Hill, J., Megier, J., \& Mehl, W. (1995). Land degradation, soil erosion and desertification monitoring in Mediterranean ecosystems. Remote Sensing Reviews, 12(1-2), 107-130.

Hill, J., Mehl, W., \& Altherr, M. (1994). Land degradation and soil erosion mapping in a Mediterranean ecosystem. In Imaging spectrometry - a tool for environmental observations (pp. 237-260). Springer Netherlands. 
Hill, J., \& Schütt, B. (2000). Mapping complex patterns of erosion and stability in dry Mediterranean ecosystems. Remote Sensing of Environment, 74(3), 557-569.

Hill, J., Stellmes, M., Udelhoven, T., Röder, A., \& Sommer, S. (2008).

Mediterranean desertification and land degradation: mapping related land use change syndromes based on satellite observations. Global and Planetary Change, 64(3), 146-157.

Hillel, D. (1980). Fundamentals of soil physics. Academic Press, Inc.(London) Ltd..

Hillel, D. (1991). Out of the Earth: Civilization and the Life of the Soil, p.4. California, U.S.: University of California Press.

Hopmans, J. W., Nielsen, D. R., \& Bristow, K. L. (2002). How useful are smallscale soil hydraulic property measurements for large-scale vadose zone modeling? Geophysical Monograph Series, 129, 247-258.

Horn, B. \& Woodham, R. (1978). Destriping LANDSAT MSS images by histogram modification. Computer Graphics and Image Processing, 10, 69-83.

Horn, R., \& Baumgartl, T. (2002). Dynamic properties of soils. Soil physics companion, 389.

Huete, A. R., \& Escadafal, R. (1991). Assessment of biophysical soil properties through spectral decomposition techniques. Remote Sensing of Environment, 35(2), 149-159.

Huete, A. R., Jackson, R. D., \& Post, D. F. (1985). Spectral response of a plant canopy with different soil backgrounds. Remote sensing of environment, 17(1), 3753.

Hunt, G. R., \& Salisbury, J. W. (1971). Visible and near infrared spectra of minerals and rocks. II. Carbonates. Modern Geology, 2, 23-30.

Hurcom, S. J., \& Harrison, A. R. (1998). The RENDVI and spectral decomposition for semi-arid vegetation abundance estimation. International Journal of Remote Sensing, 19(16), 3109-3125.

Hutchinson, C. F. (1982). Techniques for combining Landsat and ancillary data for digital classification improvement. Photogrammetric Engineering and Remote Sensing, 48, 123-130. 
Irons, J., Weismiller, R., \& Peterson, W.(1989). Soil reflectance. In G. Asrar (Ed.), Theory and applications of optical remote sensing. New York: Wiley Interscience.

Irvin, B. J., Ventura, S. J., \& Slater, B. K. (1995). Landform classification for soillandscape studies. In User Conference Proceedings, Environmental Systems Research Institute, Inc.. Redlands, CA.

ITC. (2014). ITC's Database of Satellites and Sensors. Retrieved from: http://www.itc.nl/research/products/sensordb/allsensors.aspx.

IUSS Working Group WRB. (2006). World Reference Base for Soil Resources 2006. World Soil Resource Reports (Report no. 103). Rome, FAO.

Jäger, S., \& Rickson, R. J. (1994). Modelling regional soil erosion susceptibility using the Universal Soil Loss Equation and GIS. In Conserving soil resources: European perspectives. Selected papers from the First International Congress of the European Society for Soil Conservation. (pp. 161-177). CAB INTERNATIONAL.

Janik, L. J., Merry, R. H., \& Skjemstad, J. O. (1998). Can mid infrared diffuse reflectance analysis replace soil extractions?. Animal Production Science, 38(7), 681696.

Janssen, L. L., \& van der Wel, F. J. (1994). Accuracy assessment of satellite derived land-cover data: a review. Photogrammetric engineering and remote sensing, 60(4), 419-426.

Jenny, H. (1941). Factors of soil formation. Dover Publications Inc.: New York, NY.

Jensen, J. (2005). Introductory Digital Image Processing: A Remote Sensing Perspective. (No. Ed. 3). Prentice-Hall Inc..

Justice, C. \& Townshend, J. (1981). Integrating Ground Data with Remote Sensing. Terrain Analysis and Remote Sensing, 38-58.

Kastner, C. J., \& Slater, P. N. (1983). In-flight radiometric calibration of advanced remote sensing systems. In 26th Annual Technical Symposium, 158-165. International Society for Optics and Photonics.

Kaufmann et al. (2012). Science Plan of the Environmental Mapping and Analysis Program (EnMAP). Potsdam, Germany: Deutsches GeoForschungs Zentrum. 
Kaufmann, H., Segl, K., Chabrillat, S., Hofer, S., Stuffier, T., Mueller, A., . . Bach, H. (2006). EnMAP a hyperspectral sensor for environmental mapping and analysis. Paper presented at the Geoscience and Remote Sensing Symposium, 2006. IGARSS 2006. IEEE International Conference on.

Kelcey, J., \& Lucieer, A. (2012). Sensor Correction of a 6-Band Multispectral Imaging Sensor for UAV Remote Sensing. Remote Sensing, 4(5).

Kennedy, R. E., Cohen, W. B., \& Takao, G. (1997). Empirical methods to compensate for a view-angle-dependent brightness gradient in AVIRIS imagery. Remote Sensing of Environment, 62(3), 277-291.

Kerekes, J. P., \& Landgrebe, D. A. (1988). Simulation of Optical Remote Sensing for Earth Resource Analysis. Proceedings of IGARSS ' 88 Symposium, (4).

Keshava, N. (2003). A Survey of Spectral Unmixing Algorithms. Lincoln Laboratory Journal, 14(1), 55-78.

Keshava, N., \& Mustard, J. F. (2002). Spectral unmixing. Signal Processing Magazine, IEEE, 19(1), 44-57.

Khorram, S. (1999). Accuracy assessment of remote sensing-derived change detection. Ethesda, MD: Asprs Pubns.

Kim, S. J., \& Pollefeys, M. (2008). Robust radiometric calibration and vignetting correction. Pattern Analysis and Machine Intelligence, IEEE Transactions on, 30(4), 562-576.

King, C., Baghdadi, N., Lecomte, V., \& Cerdan, O. (2005). The application of remote-sensing data to monitoring and modelling of soil erosion. Catena, 62(2), 79-93.

Konecny, G. (1972). Geometrical Aspects of Remote Sensing. Paper presented at the Int. Congress of Photogrammetry, Commission IV. Ottawa, Ontario.

Kosmas, C., Gerontidis, S., \& Marathianou, M. (2000). The effect of land use change on soils and vegetation over various lithological formations on Lesvos (Greece). Catena, 40(1), 51-68.

Kruse, F. (1989). Spectral Mapping with Landsat Thematic Mapper and Imaging Spectroscopy for Precious Metals Exploration. Proceedings of the Seventh Thematic Conference on Remote Sensing for Exploration Geology, 17-28. Calgary, Alberta. 
Kruse, F. A., Lefkoff, A. B., Boardman, J. W., Heidebrecht, K. B., Shapiro, A. T., Barloon, P. J., \& Goetz, A. F. H. (1993). The spectral image processing system (SIPS) - interactive visualization and analysis of imaging spectrometer data. Remote sensing of environment, 44(2), 145-163.

Lacaze, B., Caselles, V., Coll, V., Hill, H., Hoff, C., De Jong, S., ... Valor, E. (1996). DeMon - Integrated approaches to desertification mapping and monitoring in the Mediterranean basin. In J. Hill (Ed.), Final Report of DeMon-1 Project. Ispra (VA), Italy: Joint Research Centre of European Commission.

Lagacherie, P., Baret, F., Feret, J. B., Madeira Netto, J., \& Robbez-Masson, J. M. (2008). Estimation of soil clay and calcium carbonate using laboratory, field and airborne hyperspectral measurements. Remote Sensing of Environment, 112(3), 825835.

Lal, R. (1993). Tillage effects on soil degradation, soil resilience, soil quality, and sustainability. Soil and Tillage Research, 27(1), 1-8.

Lal, R. (1994). Soil Erosion Research Method. Soil and Water Conservation Society. St. Lucie Press, Delray Beach, FL.

Lal, R. (1998). Soil erosion impact on agronomic productivity and environment quality. Critical Reviews in Plant Sciences, 17(4), 319-464.

Lal, R. (2001). Soil degradation by erosion. Land degradation $\mathcal{E}$ development, 12(6), $519-539$.

Lal, R. (2013). Soils and Ecosystem Services. In Ecosystem Services and Carbon Sequestration in the Biosphere, 11-38. Springer Netherlands.

Lam, N. S. N., \& Quattrochi, D. A. (1992). On the Issues of Scale, Resolution, and Fractal Analysis in the Mapping Sciences*. The Professional Geographer, 44(1), 8898.

Lamp, J., \& Kneib, W. (1981). Zur quantitativen Erfassung und Bewertung von Pedofunktionen. Mitteilungen der Deutschen Bodenkundlichen Gesellschaft, 32, 695711.

Landgrebe, D. A., \& Malaret, E. R. I. C. K. (1986). Noise in remote-sensing systems: The effect on classification error. IEEE transactions on geoscience and remote sensing, 24(2), 294-300. 
Lasanta, T., Garcia-Ruiz, J. M., Pérez-Rontomé, C., \& Sancho-Marcén, C. (2000). Runoff and sediment yield in a semi-arid environment: the effect of land management after farmland abandonment. Catena, 38(4), 265-278.

Lavallie, A. (2007). Reflectivity in Remote Sensing-student version. Presented at 2007 TRESTE Annual Workshop. Browning, MT.

Law, K. H., \& Nichol, J. (2004, July). Topographic correction for differential illumination effects on IKONOS satellite imagery. In proceedings: XXth congress of International Society for Photogrammetry and Remote Sensing (35), p.6. Istanbul, Turkey.

Lee, C., \& Bethel, J. (2001). Georegistration of airborne hyperspectral image data. Geoscience and Remote Sensing, IEEE Transactions on, 39(7), 1347-1351.

Lee, K. S., Lee, G. B., \& Tyler, E. J. (1988). Thematic mapper and digital elevation modeling of soil characteristics in hilly terrain. Soil Science Society of America Journal, 52(4), 1104-1107.

Lillesand, T. M., Kiefer, R. W., \& Chipman, J. W. (2004). Remote sensing and image interpretation (No. Ed. 5). John Wiley \& Sons Ltd.

Lindstrom, M.J., Nelson, W.W. and Schuhmacher, T.E. (1992). Quantifying tillage erosion rates due to moldboard plowing. Soil \& Tillage Research, 24: 243-255.

Lindstrom, M. J., Nelson, W. W., Schumacher, T. E., \& Lemme, G. D. (1990). Soil movement by tillage as affected by slope. Soil and Tillage Research, 17(3), 255-264.

Liu, X. (2008). Airborne LiDAR for DEM generation: some critical issues. Progress in Physical Geography, 32(1), 31-49.

Liu, X., \& Kafatos, M. (2005). Land-cover mixing and spectral vegetation indices. International Journal of Remote Sensing, 26(15), 3321-3327.

Liu, J. G., \& Morgan, G. L. K. (2006). FFT selective and adaptive filtering for removal of systematic noise in ETM+ imageodesy images. Geoscience and Remote Sensing, IEEE Transactions on, 44(12), 3716-3724.

Lunetta, R., Congalton, R. G., Fenstermaker, L., Jensen, J., McGwire, K. \& Tinney, L. (1991). Remote Sensing and Geographic Information System Data Integration: Error Sources and. Photogrammetric Engineering \& Remote Sensing, 57(6), 677-687. 
Malenovský, Z., Bartholomeus, H. M., Acerbi-Junior, F. W., Schopfer, J. T., Painter, T. H., Epema, G. F., \& Bregt, A. K. (2007). Scaling dimensions in spectroscopy of soil and vegetation. International Journal of Applied Earth Observation and Geoinformation, 9(2), 137-164.

Margate, D. E., \& Shrestha, D. P. (2001). The use of hyperspectral data in identifying "desert-like" soil surface features in Tabernas area, southeast Spain. In Paper presented at the 22nd Asian Conference on Remote Sensing (5), p. 9.

Martínez, P. J., Pérez, R. M., Plaza, A., Aguilar, P. L., Cantero, M. C., \& Plaza, J. (2006). Endmember extraction algorithms from hyperspectral images. Retrieved from: http:// www.earth-prints.org/handle/2122/1963.

Martínez-Casasnovas, J. A., \& Sánchez-Bosch, I. (2000). Impact assessment of changes in land use/conservation practices on soil erosion in the Penedès-Anoia vineyard region (NE Spain). Soil and Tillage Research, 57(1), 101-106.

Mather, P. M. (1999). Computer processing of remotely-sensed images: An introduction, New York, NY: Wiley.

Matheron, G. (1962). Traité de géostatistique appliquée. 1 (1962) (Vol. 1). Editions Technip.

McCoy, R. M. (2005). Field methods in remote sensing. New York, NY: Guilford Press.

McDonald, E. R., Wu, X., Caccetta, P. A., \& Campbell, N. A. (2002). Illumination correction of Landsat TM data in south east NSW. Environment Australia.

McGwire, K. C. (1996). Cross-validated assessment of geometric accuracy. Photogrammetric engineering and remote sensing, 62(10), 1179-1188.

McKenzie, N. J., \& Ryan, P. J. (1999). Spatial prediction of soil properties using environmental correlation. Geoderma, 89(1), 67-94.

Melendez-Pastor, I., Navarro-Pedreño, J., Gómez, I., \& Koch, M. (2008). Identifying optimal spectral bands to assess soil properties with VNIR radiometry in semi-arid soils. Geoderma, 147(3), 126-132.

Merrill, S. D. (1998). Comments on the chain method for measuring soil surface roughness: Use of the chain set. Soil Science Society of America Journal, 62(4), 11471149. 
Meusburger, K., Bänninger, D., \& Alewell, C. (2010). Estimating vegetation parameter for soil erosion assessment in an alpine catchment by means of QuickBird imagery. International Journal of Applied Earth Observation and Geoinformation, 12(3), 201-207.

Meyer, P., Itten, K. I., Kellenberger, T., Sandmeier, S., \& Sandmeier, R. (1993). Radiometric corrections of topographically induced effects on Landsat TM data in an alpine environment. ISPRS Journal of Photogrammetry and Remote Sensing, $48(4), 17-28$.

Minasny, B. and McBratney, A. (2014). Digital Soil Mapping: A Brief History and Lessons. Geophysical Research Abstracts. EGU General Assembly,16.

Ministerio de Agricultura, Alimentacion y Medio Ambiente (2014). Sistema de Información Geográfica de Parcelas Agrícolas. Retrieved from:

http://sigpac.mapa.es/fega/visor.

Moore, I. D., Gessler, P. E., Nielsen, G. A. E., \& Peterson, G. A. (1993). Soil attribute prediction using terrain analysis. Soil Science Society of America Journal, $57(2), 443-452$.

Moran, M. S., Hymer, D. C., Qi, J., \& Kerr, Y. (2002). Comparison of ERS-2 SAR and Landsat TM imagery for monitoring agricultural crop and soil conditions. Remote Sensing of Environment, 79(2), 243-252.

Morgan, R. P. C. (1995). Soil erosion and conservation. (2nd Ed.). John Wiley \& Sons. Morgan, R.P.C., Morgan, D.D.V. and Finney H. (1984). A Predictive Model for the Assessment of Soil Erosion Risk. Journal of Agricultural Engineering Research (30), 245-253.

Morgan, R. P. C., Quinton, J. N., \& Rickson, R. J. (1992). EUROSEM documentation manual. Silsoe, UK: Silsoe College.

Morvan, X., Saby, N. P. A., Arrouays, D., Le Bas, C., Jones, R. J. A., Verheijen, F. G. A., ... \& Kibblewhite, M. G. (2008). Soil monitoring in Europe: a review of existing systems and requirements for harmonisation. Science of the total environment, 391(1), 1-12.

Mosier, A. R. (1998). Soil processes and global change. Biology and fertility of soils, 27(3), 221-229. 
Myint, S. W., \& Okin, G. S. (2009). Modelling land-cover types using multiple endmember spectral mixture analysis in a desert city. International Journal of Remote Sensing, 30(9), 2237-2257.

Nagler, P. L., Inoue, Y., Glenn, E. P., Russ, A. L., \& Daughtry, C. S. T. (2003). Cellulose absorption index (CAI) to quantify mixed soil-plant litter scenes. Remote Sensing of Environment, 87(2), 310-325.

National Aeronautics and Space Administration. (2011). Landsat 7 Science Data Users Handbook. Retrieved from: http://landsathandbook.gsfc.nasa.gov/data_artifacts/prog_sect7_8.html.

National Oceanic and Atmospheric Administration. (2013). LIDAR-Light Detection and Ranging -is a remote sensing method used to examine the surface of earth. Retrieved from: http://oceanservice.noaa.gov/facts/lidar.html.

Natural Resources Canada. (2008). Geometric Distortion in Imagery. Retrieved from: http://www.nrcan.gc.ca/earth-sciences/geography-boundary/remotesensing/fundamentals/2309.

Natural Resources Canada. (2013). What is Remote Sensing? Retrieved from: http://www.nrcan.gc.ca/earth-sciences/geomatics/satellite-imagery-airphotos/satellite-imagery-products/educational-resources/9379.

Natural Resources Canada. (2013a). Radiometric Resolution. Retrieved from: https://www.nrcan.gc.ca/earth-sciences/geomatics/satellite-imagery-airphotos/satellite-imagery-products/educational-resources/9493

Natural Resources Canada. (2013b). Spectral Resolution. Retrieved from: http://www.nrcan.gc.ca/earth-sciences/geomatics/satellite-imagery-airphotos/satellite-imagery-products/educational-resources/9393.

Nature Geoscience. (2010). Editorial: Investing in Soils. Nature Geoscience, 3, p. 295.

Nearing, M. A., Foster, G. R., Lane, L. J., \& Finkner, S. C. (1989). A process-based soil erosion model for USDA-Water Erosion Prediction Project technology. Trans. ASAE, 32(5), 1587-1593.

Neville, R. A., Sun, L., \& Staenz, K. (2008). Spectral calibration of imaging spectrometers by atmospheric absorption feature matching. Canadian Journal of Remote Sensing, 34 (S1), S29-S42. 
Nicodemus, F. E. (1977). Geometrical considerations and nomenclature for reflectance (160). Washington, D. C: US Department of Commerce, National Bureau of Standards.

Nkemakilam, I. (2013). Geometric correction, orthorectification and mosaicking. St. Catharine, ON: Ogis Geoinfo Inc..

Numata, I., Roberts, D. A., Chadwick, O. A., Schimel, J., Sampaio, F. R., Leonidas, F. C., \& Soares, J. V. (2007). Characterization of pasture biophysical properties and the impact of grazing intensity using remotely sensed data. Remote Sensing of Environment, 109(3), 314-327.

Odeh, I. O., \& McBratney, A. B. (2000). Using AVHRR images for spatial prediction of clay content in the lower Namoi Valley of eastern Australia. Geoderma, 97(3), 237-254.

Odeh, I.O., McBratney, A.B., Chittleborough, D. (1993). Spatial prediction of soil properties from landform attributes derived form a digital elevation model. Geoderma, 63, 197-214.

Okin, G. S., Roberts, D. A., Murray, B., \& Okin, W. J. (2001). Practical limits on hyperspectral vegetation discrimination in arid and semiarid environments. Remote Sensing of Environment, 77(2), 212-225.

Oost van, K., Govers, G., \& Desmet, P. (2000). Evaluating the effects of changes in landscape structure on soil erosion by water and tillage. Landscape ecology, 15(6), 577-589.

Ortega,Varela, C., \& Simó, A. (2007). Facilitating the CAP reform: compliance and competitiveness of European agriculture. STRP working document D-5 Spain.

Paine, D. P. (1981). Aerial photography and image interpretation for resource management. Wiley Publishing.

Palacios-Orueta, A., Pinzon, J. E., Ustin, S. L., \& Roberts, D. A. (1999). Remote sensing of soils in the Santa Monica Mountains: II. Hierarchical foreground and background analysis. Remote Sensing of Environment, 68(2), 138-151.

Palacios-Orueta, A., \& Ustin, S. L. (1998). Remote sensing of soil properties in the Santa Monica Mountains I. Spectral analysis. Remote Sensing of Environment, 65(2), 170-183. 
Palubinskas, G., Muller, R., \& Reinartz, P. (2003, July). Mosaicking of optical remote sensing imagery. In Geoscience and Remote Sensing Symposium, 2003. IGARSS' 03. Proceedings. 2003 IEEE International (6), 3955-3957. IEEE.

Parente, M., Clark, J., Brown, A. J., \& Bishop, J. L. (2010). End-to-end simulation and analytical model of remote-sensing systems: Application to CRISM. Geoscience and Remote Sensing, IEEE Transactions on, 48(11), 3877-3888.

Peddle, D. R., Hall, F. G., \& LeDrew, E. F. (1999). Spectral mixture analysis and geometric-optical reflectance modeling of boreal forest biophysical structure. Remote Sensing of Environment, 67(3), 288-297.

Peisker, T., Spengler, D., Segl, K., Hostert, P., \& Kaufmann, H. (2010). Simulation of enmap cereal canopy spectra- challenges posed by varying observation geometry and plant phenology. proceeding of the hyperspectral Workshop 2010, 1719 March 2010 ESA-ESRIN, Frascati, Italy.

Pimentel, D. (Ed.). (1993). World soil erosion and conservation. Cambridge: Cambridge University Press.

Pimentel, D. (2001).The limitations of Biomass Energy. In Encyclopedia of Physical Science and Technology, Academic Press, San Diego,CA, pp. 159-171.

Pimentel, D. (2006). Soil Erosion A Food and Environmental Threat. Environment, Development and Sustainability, 8: 119-137.

Pimentel, D., Harvey, C., Resosudarmo, P., Sinclair, K., Kurz, D., McNair, M., ... \& Blair, R. (1995). Environmental and economic costs of soil erosion and conservation benefits. Science-AAAS-Weekly Paper Edition, 267(5201), 1117-1122.

Pimentel, D. and Kounang, N. (1998). Ecology of Soil Erosion in Ecosystems. Ecosystems, 1: 416-426.

Plaza, A., Benediktsson, J. A., Boardman, J. W., Brazile, J., Bruzzone, L., CampsValls, G., ... \& Trianni, G. (2009). Recent advances in techniques for hyperspectral image processing. Remote Sensing of Environment, 113, S110-S122.

Plaza, A., Martínez, P., Pérez, R., \& Plaza, J. (2004). A quantitative and comparative analysis of endmember extraction algorithms from hyperspectral data. Geoscience and Remote Sensing, IEEE Transactions on, 42(3), 650-663. 
Podmore, T. H., \& Huggins, L. F. (1981). An automated profile meter for surface roughness measurements [for tillage effectiveness]. Transactions of the ASAE [American Society of Agricultural Engineers](USA).

Poesen, J. W. A., \& Hooke, J. M. (1997). Erosion, flooding and channel management in Mediterranean environments of southern Europe. Progress in Physical Geography, 21(2), 157-199.

Powell, R. L., Roberts, D. A., Dennison, P. E., \& Hess, L. L. (2007). Sub-pixel mapping of urban land cover using multiple endmember spectral mixture analysis: Manaus, Brazil. Remote Sensing of Environment, 106(2), 253-267.

Puech, C. (1994). Thresholds of homogeneity in targets in the landscape. Relationship with remote sensing. International Journal of Remote Sensing, 15(12), 2421-2435.

Pugnaire, F. I., Luque, M. T., Armas, C., \& Gutierrez, L. (2006). Colonization processes in semi-arid Mediterranean old-fields. Journal of Arid Environments, 65(4), 591-603.

Puigdefábregas, J., \& Mendizabal, T. (1998). Perspectives on desertification: western Mediterranean. Journal of Arid Environments, 39(2), 209-224.

Raber, G. T., Jensen, J. R., Hodgson, M. E., Tullis, J. A., Davis, B. A., \& Berglund, J. (2007). Impact of LiDAR nominal post-spacing on DEM accuracy and flood zone delineation. Photogrammetric Engineering and Remote Sensing, 73(7), 793.

Raina, P., Joshi, D. C., \& Kolarkar, A. S. (1993). Mapping of soil degradation by using remote sensing on alluvial plain, Rajasthan, India. Arid Land Research and Management, 7(2), 145-161.

Rapp, A. (1986). Introduction to soil degradation processes in drylands. Climatic change, 9(1-2), 19-31.

Ray, T. W., \& Murray, B. C. (1996). Nonlinear spectral mixing in desert vegetation. Remote sensing of environment, 55(1), 59-64.

Reed, M. S., Buenemann, M., Atlhopheng, J., Akhtar-Schuster, M., Bachmann, F., Bastin, G., ... \& Verzandvoort, S. (2011). Cross-scale monitoring and assessment of land degradation and sustainable land management: A methodological framework for knowledge management. Land Degradation $\mathcal{E}$ Development, 22(2), 261-271. 
Reeves, J. B., McCarty, G. W., \& Meisinger, J. J. (1999). Near infrared reflectance spectroscopy for the analysis of agricultural soils. Journal of Near Infrared Spectroscopy, 7(3), 179-193.

Reif, M., C. Piercy, J. Jarvis, B. Sabol, C. Macon, R. Loyd, P. Colarusso, H. Dierssen, and J. Aitken. 2012. Ground truth sampling to support remote sensing research and development: Submersed aquatic vegetation species discrimination using an airborne hyperspectral/lidar system. DOER Technical Notes Collection. ERDC TN-DOERE30.

Vicksburg, MS: U.S. Army Engineer Research and Development Center.

Reynolds, J. F., Smith, D. M. S., Lambin, E. F., Turner, B. L., Mortimore, M., Batterbury, S. P., ... \& Walker, B. (2007). Global desertification: building a science for dryland development. science, 316(5826), 847-851.

Richards, J.A. (1993). Remote Sensing Digital Image Analysis, An Introduction. New York: Springer-Verlag.

Richards, J. A. (2013). Remote Sensing Digital Image Analysis: An Introduction (5th ed.). Heidelberg, Germany: Springer.

Richards, J. F. (1990). Land transformation. The earth as transformed by human action, 163-178.

Richards, J. A., Landgrebe, D. A., \& Swain, P. H. (1982). A means for utilizing ancillary information in multispectral classification. Remote Sensing of Environment, 12(6), 463-477.

Richter, R. (2004). ATCOR: Atmospheric and Topographic Correction. DLR German Aerospace Center.

Richter, R., \& Schläpfer, D. (2002). Geo-atmospheric processing of airborne imaging spectrometry data. Part 2: atmospheric/topographic correction. International Journal of Remote Sensing, 23(13), 2631-2649.

Richter, R., \& Schläpfer, D. (2012). Atmospheric/Topographic Correction for Airborne Imagery. ATCOR-4 User Guide. In DLR (Ed.).

Roberts, D. A., Dennison, P. E., Gardner, M. E., Hetzel, Y., Ustin, S. L., \& Lee, C. T. (2003). Evaluation of the potential of Hyperion for fire danger assessment by comparison to the Airborne Visible/Infrared Imaging Spectrometer. Geoscience and Remote Sensing, IEEE Transactions on, 41(6), 1297-1310. 
Roberts, D. A., Gardner, M., Church, R., Ustin, S., Scheer, G., \& Green, R. O. (1998). Mapping chaparral in the Santa Monica Mountains using multiple endmember spectral mixture models. Remote Sensing of Environment, 65(3), 267279.

Roberts, D., Halligan, K., \& Dennison, P. (2007). VIPER Tools user manual. Univ. of Calif., Santa Barbara, California.

Roberts, D. A., Smith, M. O., \& Adams, J. B. (1993). Green vegetation, nonphotosynthetic vegetation, and soils in AVIRIS data. Remote Sensing of Environment, 44(2), 255-269.

Roberts, D. A., Yamaguchi, Y., \& Lyon, R. J. P. (1986). Comparison of various techniques for calibration of AIS data. NASA STI/Recon Technical Report N, 87, 12970.

Robertson, G. P., Crum, J. R., \& Ellis, B. G. (1993). The spatial variability of soil resources following long-term disturbance. Oecologia, 96(4), 451-456.

Rocchini, D., \& Di Rita, A. (2005). Relief effects on aerial photos geometric correction. Applied Geography, 25(2), 159-168.

Rogaß, Christian, Daniel Spengler, Mathias Bochow, Karl Segl, Angela Lausch, Daniel Doktor, Sigrid Roessner, Robert Behling, Hans-Ulrich Wetzel, and Hermann Kaufmann. (2011). Reduction of radiometric miscalibration applications to pushbroom sensors. Sensors, 11(6), 6370-6395.

Rogge, D., Bachmann, M., Rivard, B., \& Feng, J. (2012, July). Hyperspectral flightline leveling and scattering correction for image mosaics. In Geoscience and Remote Sensing Symposium (IGARSS), 2012 IEEE International, 4094-4097. IEEE.

Sandberg, R. L., Richtsmeier, S., \& Haren, R. (2005). Full optical spectrum hyperspectral scene simulation. SPECTRAL SCIENCES INC BURLINGTON MA.

Sandmeier, S., \& Itten, K. I. (1997). A physically-based model to correct atmospheric and illumination effects in optical satellite data of rugged terrain. Geoscience and Remote Sensing, IEEE Transactions on, 35(3), 708-717.

Sandmeier, S., Müller, C., Hosgood, B., \& Andreoli, G. (1998). Sensitivity analysis and quality assessment of laboratory BRDF data. Remote Sensing of Environment, 64(2), 176-191. 
Sauer, T., \& Ries, J. B. (2008). Vegetation cover and geomorphodynamics on abandoned fields in the Central Ebro Basin (Spain). Geomorphology, 102(2), 267277.

Schaepman, M., Schläpfer, D., Müller, A., \& Strobl, P. (1997). Ground Spectroradiometric Measurements in Support of the Validation of the Calibration of DIGITAL Airborne Imaging Spectrometer (DAIS 7915) Data. In Presented at the Third International Airborne Remote Sensing Conference and Exhibition (7), p. 10.

Schiefer, S., Hostert, P., \& Damm, A. (2006). Correcting brightness gradients in hyperspectral data from urban areas. Remote Sensing of Environment, 101(1), 25-37.

Schläpfer, D., Hausold, A., \& Richter, R. (2000). A Unified Approach to Parametric Geocoding and Atmospheric/Topographic Correction for Wide FOV Airborne Imagery Part 1: Parametric Ortho-Rectification Process. In Proc. 2nd EARSeL Workshop on Imaging Spectroscopy, EARSeL, Enschede (Vol. 9).

Schläpfer, D., \& Richter, R. (2002). Geo-atmospheric processing of airborne imaging spectrometry data. Part 1: parametric orthorectification. International Journal of Remote Sensing, 23(13), 2609-2630.

Schläpfer, D., Schaepman, M. E., \& Itten, K. I. (1998). PARGE: Parametric geocoding based on GCP-calibrated auxiliary data. In SPIE's International Symposium on Optical Science, Engineering, and Instrumentation, 334-344. International Society for Optics and Photonics.

Schläpfer, D., Schaepman, M., \& Strobl, P. (2001). Impact of spatial resampling methods on the radiometric accuracy of airborne imaging spectrometer data. In Proc. 5th Int. Airborne Remote Sens. Conf. Exhib.

Schlesinger, W. H., \& Pilmanis, A. M. (1998). Plant-soil interactions in deserts. In Plant-induced soil changes: Processes and feedbacks (pp. 169-187). Springer

Netherlands.

Schlesinger, W. H., Reynolds, J. F., Cunningham, G. L., Huenneke, L. F., Jarrell, W. M., Virginia, R. A., \& Whitford, W. G. (1990). Biological feedbacks in global desertification. Science (Washington), 247(4946), 1043-1048.

Schmid, T., Palacios-Orueta, A., Chabrillat, S., Ben-Dor, E., Plaza, A., Rodriguez, M., .. Cicuendez., V. (2012). Spectral Characteristics of Land Surface Composition to Determine Soil Erosion within Semiarid Rainfed Cultivated Areas. Paper presented at the IGARSS 2012. Munich, Germany. 
Schmidtlein, S., Zimmermann, P., Schupferling, R. \& Weiss, C. (2007). Mapping the Floristic Continuum: Ordination Space Position Estimated from Imaging Spectroscopy. Journal of Vegetation Science, 18, 131-140.

Schott, J. R., Salvaggio, C., \& Volchok, W. J. (1988). Radiometric scene normalization using pseudoinvariant features. Remote Sensing of Environment, 26(1), 1-16.

Schowengerdt, R. A. (2006). Remote sensing: models and methods for image processing. Academic press.

Scull, P., Franklin, J., Chadwick, O. A., \& McArthur, D. (2003). Predictive soil mapping: a review. Progress in Physical Geography, 27(2), 171-197.

Segl, K., Guanter, L., Kaufmann, H., Schubert, J., Kaiser, S., Sang, B., \& Hofer, S. (2010). Simulation of spatial sensor characteristics in the context of the EnMAP hyperspectral mission. Geoscience and Remote Sensing, IEEE Transactions on, 48(7), 3046-3054.

Segl, K., Guanter, L., Rogass, C., Kuester, T., Roessner, S., Kaufmann, H., ... \& Hofer, S. (2012). EeteS - The EnMAP end-to-end simulation tool. Selected Topics in Applied Earth Observations and Remote Sensing, IEEE Journal of, 5(2), 522-530.

Semiarid. (n.d.). Dictionary.com Unabridged. Retrieved April 20, 2014, from Dictionary.com website: http:// dictionary.reference.com/browse/semiarid.

Serbin, G., Daughtry, C. S., Hunt Jr, E. R., Reeves III, J. B., \& Brown, D. J. (2009). Effects of soil composition and mineralogy on remote sensing of crop residue cover. Remote Sensing of Environment, 113(1), 224-238.

Settle, J. J., \& Drake, N. A. (1993). Linear mixing and the estimation of ground cover proportions. International Journal of Remote Sensing, 14(6), 1159-1177.

Sever, T. (n.d). The Peten, Guatemala- Ground-Truth Information. Retrieved from: http://weather.msfc.nasa.gov/archeology/peten_groundtruth.html.

Shang, J., Neville, R., Staenz, K., Sun, L., Morris, B., \& Howarth, P. (2008). Comparison of fully constrained and weakly constrained unmixing through mine-tailing composition mapping. Canadian Journal of Remote Sensing, 34(S1), S92-S109.

Shepherd, K. D., \& Walsh, M. G. (2002). Development of reflectance spectral libraries for characterization of soil properties. Soil Science Society of America Journal, 66(3), 988-998.

Shippert, P. (2002). Spotlight on hyperspectral. Geospatial Solutions, 12, 40-45. 
Shrestha, D. P., Margate, D. E., Meer, F. V. D., \& Anh, H. V. (2005). Analysis and classification of hyperspectral data for mapping land degradation: An application in southern Spain. International Journal of Applied Earth Observation and Geoinformation, 7(2), 85-96.

Simonson, R. W. (1959). Outline of a generalized theory of soil genesis. Soil Science Society of America Journal, 23(2), 152-156.

Singer, R. B., \& McCord, T. B. (1979). Mars-Large scale mixing of bright and dark surface materials and implications for analysis of spectral reflectance. In Lunar and Planetary Science Conference Proceedings (10), 1835-1848.

Smedes, H. W. (1975). The truth about ground truth. In Proceedings 10th International Symposium on Remote Sensing of Environment, 821-823.

Smith, J. A., Lin, T. L., \& Ranson, K. L. (1980). The Lambertian assumption and Landsat data. Photogrammetric Engineering and Remote Sensing, 46(9), 1183-1189.

Smith, G. M., \& Milton, E. J. (1999). The use of the empirical line method to calibrate remotely sensed data to reflectance. International Journal of Remote Sensing, 20(13), 2653-2662.

Smith, M. O., Ustin, S. L., Adams, J. B., \& Gillespie, A. R. (1990). Vegetation in deserts: I. A regional measure of abundance from multispectral images. Remote sensing of Environment, 31(1), 1-26.

Soil Survey Staff. (1999). Soil Taxonomy, A Basic System of Soil Classification of Making and Interpreting Soil Surveys (pp. 869). Washington, D.C.: Natural Resources Conservation Service, USDA.

Soil Survey Staff. (2010). Keys to Soil Taxonomy (11 th ed.). Washington, DC: USDANatural Resources Conservation Service.

Song, C., Woodcock, C. E., Seto, K. C., Lenney, M. P., \& Macomber, S. A. (2001). Classification and change detection using Landsat TM data: when and how to correct atmospheric effects?. Remote sensing of Environment, 75(2), 230-244.

Song, C. (2005). Spectral mixture analysis for subpixel vegetation fractions in the urban environment: How to incorporate endmember variability?. Remote Sensing of Environment, 95(2), 248-263.

Staenz, K., Budkewitsch, P., Neville, R. A., Hitchcock, R., \& Nadeau, C. (2001). Spectral unmixing of rock/mineral targets based on different spatial resolution 
hyperspectral data. Paper presented at the ISSSR'01: Proceedings of the International Symposium on Spectral Sensing Research.

Staenz, K., Gauthier, R. P., Teillet, P. M., \& Williams, D. J. (1993, September). Bidirectional reflectance effects derived from ASAS imagery of a pecan orchard. In Optical Engineering and Photonics in Aerospace Sensing (pp. 32-42). International Society for Optics and Photonics.

Staenz, K., \& Williams, D. J. (1997). Retrieval of surface reflectance from hyperspectral data using a look-up table approach. Canadian Journal of Remote Sensing, 23(4), 354-368.

Stehman, S. V. (1997). Selecting and interpreting measures of thematic classification accuracy. Remote sensing of Environment, 62(1), 77-89.

Stenberg, B., Viscarra Rossel, R. A., Mouazen, A. M., \& Wetterlind, J. (2010). Chapter Five-Visible and Near Infrared Spectroscopy in Soil Science. Advances in agronomy, 107, 163-215.

Stevenson, A. \& Harrison, R. (1992). Ancient Forests in Spain: A Model for Landuse and Dry Forest Management in South-East Spain from 4000BC to 1900AD. Prehistoric Society (58), 227-247.

Stewart, B. and Lal, R. (1992). Advances in Soil Science, Soil Science, 11(3).

Stewart, B. A., Lal, R., \& El-Swaify, S. A. (1991). Sustaining the resource base of an expanding world agriculture. IN: Soil Management for Sustainability, 125-144. Ankeny, Iowa: Soil and Water Conservation Society.

Stoner, E., Baumgardner, M., Biehl, L. \& Robinson, B. (1980). Atlas of soil reflectance properties. West Lafayette, Purdue University.

Stuffler, T.; Förster, K.; Hofer, S.; Leipold, M.; Sand, B.; Kaufmann, H.; Penné, B.; Müller, A.; Chlebek, C. (2008): Hyperspectral Imaging-An advanced instrument concept for the EnMAP mission (Environmental mapping and analysis programme). 59th International Astronautical Congress 2008, Earth Observation Symposium, Earth Observation Sensors \& Technology, International Astronautical Federation, pp. AC-08.B1.3.4.

Sun, L., Neville, R., Staenz, K., \& White, H. P. (2008). Automatic destriping of Hyperion imagery based on spectral moment matching. Canadian Journal of Remote Sensing, 34(S1), S68-S81. 
Symeonakis, E., Calvo-Cases, A. and Arnau-Rosalen, E. (2007). Land Use Change and Land Degradation in Southeastern Mediterranean Spain. Environmental Management, 40: 80-94.

Teillet, P. M. (1986). Image correction for radiometric effects in remote sensing. International Journal of Remote Sensing, 7(12), 1637-1651.

Teillet, P. M. (1997). A status overview of earth observation calibration/validation for terrestrial applications. Canadian journal of remote sensing, 23(4), 291-298.

Teillet, P. M., Barker, J. L., Markham, B. L., Irish, R. R., Fedosejevs, G., \& Storey, J. C. (2001). Radiometric cross-calibration of the Landsat-7 ETM+ and Landsat-5 TM sensors based on tandem data sets. Remote Sensing of Environment, 78(1), 3954.

Teillet, P. \& Coburn, C. (2010). Radiometric Correction. Retrieved from SAGE publications: http://www.sage-ereference.com/geography/Article_n952.html.

Teillet, P. M., Guindon, B., \& Goodenough, D. G. (1982). On the slope-aspect correction of multispectral scanner data. Canadian Journal of Remote Sensing, 8(2), 84-106.

Thiemann, S., \& Kaufmann, H. (2002). Lake water quality monitoring using hyperspectral airborne data - a semiempirical multisensor and multitemporal approach for the Mecklenburg Lake District, Germany. Remote Sensing of Environment, 81(2), 228-237.

Thierry, B., \& Lowell, K. (2001). An uncertainty-based method of photointerpretation. Photogrammetric engineering and remote sensing, 67(1), 65-72.

Thomlinson, J. R., Bolstad, P. V., \& Cohen, W. B. (1999). Coordinating methodologies for scaling landcover classifications from site-specific to global: Steps toward validating global map products. Remote Sensing of Environment, 70(1), 16-28.

Thornes, J. B., \& Wainwright, J. (2003). Environmental issues in the Mediterranean: processes and perspectives from the past and present, (1). Routledge.

Tian, Y., Woodcock, C. E., Wang, Y., Privette, J. L., Shabanov, N. V., Zhou, L., ... \& Myneni, R. B. (2002). Multiscale analysis and validation of the MODIS LAI product: I. Uncertainty assessment. Remote Sensing of Environment, 83(3), 414-430. 
Toky, O. P., \& Ramakrishnan, P. S. (1983). Secondary succession following slash and burn agriculture in North-Eastern India: I. biomass, litterfall and productivity. The Journal of Ecology, 735-745.

Tompkins, S., Mustard, J. F., Pieters, C. M., \& Forsyth, D. W. (1997). Optimization of endmembers for spectral mixture analysis. Remote Sensing of Environment, 59(3), 472-489.

Torrent, J., \& Barrón, V. (1993). Laboratory measurement of soil color: theory and practice. Soil color, (soilcolor), 21-33.

Toutin, T. (2004). Review article: Geometric processing of remote sensing images: models, algorithms and methods. International Journal of Remote Sensing, 25(10), 1893-1924.

Townsend, P. A. (2000). A quantitative fuzzy approach to assess mapped vegetation classifications for ecological applications. Remote Sensing of environment, 72(3), 253-267.

Townshend, J. R., Justice, C. O., Gurney, C., \& McManus, J. (1992). The impact of misregistration on change detection. Geoscience and Remote Sensing, IEEE Transactions on, 30(5), 1054-1060.

Trodd, N. M. (1995). Uncertainty in land cover mapping for modelling land cover change. Proceedings RSS95: Remote Sensing in Action, 1138-1145.

Troeh, F. R. (1991). Soil and Water Conservation Englewood Cliffs, NJ: Prentice Hall.

Tromp, M., \& Epema, G. F. (1998). Spectral mixture analysis for mapping land degradation in semi-arid areas. Geologie en Mijnbouw, 77(2), 153-160.

Tsai, F. \& Chen, W. (2008). Striping Noise Detection and Correction of Remote Sensing Images. Geoscience and Remote Sensing, IEEE Transactions on, 46(12), 41224131.

Tseng, Y. H. (2000). Spectral unmixing for the classification of hyperspectral images. International Archives of Photogrammetry and Remote Sensing, 33(B7/4; PART 7), 1532-1538.

Tueller, P. T. (1987). Remote sensing science applications in arid environments. Remote Sensing of Environment, 23(2), 143-154. 
UNCCD. (2000). Assessment of the Status of Land Degradation in Arid, Semi-Arid and Dry Sub-Humid Areas. Bonn: United Nations Convention to Combat Desertification.

UNEP. (1988). First United Nations Global Assessment of Human Induced Soil Degradation. Retrieved from:

http://www.isric.org/sites/default/files/ExplanNote_1.pdf.

UNEP. (2012). The Fifth Global Environmental Outlook Report-Chapter3: LAND. United Nations Environment Programme.

UNEP.(2013). Land Degradation. Retrieved from:

http:// www.unep.org/dgef/LandDegradation/tabid/1702/Default.aspx.

United Nations. (1994). UN Earth Summit. Convention on desertification. Paper presented at UN Conference in Environment and Development. Rio de Janeiro, Brazil.

United States Department of Agriculture. Office of Public Affairs. (1990). Fact book of agriculture, (1063). Office of Public Affairs, US Dept. of Agriculture.

USGS. (2011). Deriving Phenological Metrics from NDVI. Retrieved from http://phenology.cr.usgs.gov/methods_metrics.php.

van der Knijff, J. M., Jones, R. J. A., \& Montanarella, L. (2000). Soil erosion risk assessment in Europe, 1-38. European Soil Bureau, European Commission.

van der Meer, F. (1995). Spectral reflectance of carbonate mineral mixtures and bidirectional reflectance theory: Quantitative analysis techniques for application in remote sensing. Remote Sensing Reviews, 13(1-2), 67-94.

van der Meer, F. (2002). Image classification through spectral unmixing. In Spatial statistics for remote sensing (pp. 185-193). Springer Netherlands.

van der Meer, F., Bakker, W., Scholte, K., Skidmore, A., de Jong, S., Clevers, J., \& Epema, G. (1999). Simulation of MERIS data: potentials and limitations for mapping (soil) mineralogy. International Journal of Applied Earth Observation and Geoinformation, 1(3), 196-204.

van der Meer, F., \& De Jong, S. M. (2000). Improving the results of spectral unmixing of Landsat Thematic Mapper imagery by enhancing the orthogonality of end-members. International Journal of Remote Sensing, 21(15), 2781-2797. 
Veganzones, M. A., \& Grana, M. (2008, January). Endmember extraction methods: A short review. In Knowledge-Based Intelligent Information and Engineering Systems (pp. 400-407). Springer Berlin Heidelberg.

Verhoef, W., \& Bach, H. (2003). Simulation of hyperspectral and directional radiance images using coupled biophysical and atmospheric radiative transfer models. Remote Sensing of Environment, 87(1), 23-41.

Vermote, E. F., Tanré, D., Deuze, J. L., Herman, M., \& Morcette, J. J. (1997).

Second simulation of the satellite signal in the solar spectrum, 6S: An overview. Geoscience and Remote Sensing, IEEE Transactions on, 35(3), 675-686.

Veron, S. R., Paruelo, J. M., \& Oesterheld, M. (2006). Assessing desertification. Journal of Arid Environments, 66(4), 751-763.

Viscarra Rossel, R. A., Walvoort, D. J. J., McBratney, A. B., Janik, L. J., \& Skjemstad, J. O. (2006). Visible, near infrared, mid infrared or combined diffuse reflectance spectroscopy for simultaneous assessment of various soil properties. Geoderma, 131(1), 59-75.

Wang, F., Brent Hall, G., \& Subaryono. (1990). Fuzzy information representation and processing in conventional GIS software: database design and application. International Journal of Geographical Information System, 4(3), 261-283.

Wang, X., Chen, F., \& Dong, Z. (2006). The relative role of climatic and human factors in desertification in semiarid China. Global Environmental Change, 16(1), 48-57.

Wang, Z., Coburn, C. A., Ren, X., \& Teillet, P. M. (2012). Effect of soil surface roughness and scene components on soil surface bidirectional reflectance factor. Canadian Journal of Soil Science, 92(2), 297-313.

Warkentin, B. P. (1995). The changing concept of soil quality. Journal of Soil and Water Conservation, 50(3), 226-228.

Weber, D., \& Englund, E. (1992). Evaluation and comparison of spatial interpolators. Mathematical Geology, 24(4), 381-391.

Wegener, M. (1990). Destriping multiple sensor imagery by improved histogram matching. International Journal of Remote Sensing, 11(5), 859-875. 
Weidong, L., Baret, F., Xingfa, G., Qingxi, T., Lanfen, Z., \& Bing, Z. (2002). Relating soil surface moisture to reflectance. Remote sensing of environment, 81(2), 238-246.

Weiskel, T. C. (1989). The ecological lessons of the past: an anthropology of environmental decline. The Ecologist, 19(3), 98-103.

Weng, Q. (Ed.). (2010). Remote sensing of impervious surfaces. CRC Press.

Wilson, J. P., \& Gallant, J. C. (Eds.). (2000). Terrain analysis: principles and applications. John Wiley \& Sons.

Winter, M. (1999). N-FINDER: An algorithm for fast autonomous spectral end-member determination in hyperspectral data. Proceedings in SPIE, (3753), 266-275.

Woodcock, C. E., \& Strahler, A. H. (1987). The factor of scale in remote sensing. Remote sensing of Environment, 21(3), 311-332.

Wong, K. W. (1980). Basic mathematics of photogrammetry. Manual of photogrammetry, 4, 37-101.

World Weather and Climate Information. (2013). Average Weather in Madrid, Spain. Retrieved from: http://www.weather-and-climate.com/average-monthlyRainfall-Temperature-Sunshine,Madrid,Spain.

Yang, D., Kanae, S., Oki, T., Koike, T., \& Musiake, K. (2003). Global potential soil erosion with reference to land use and climate changes. Hydrological Processes, 17(14), 2913-2928.

Yang, L., \& Wu, J. (2010). Seven design principles for promoting scholars' participation in combating desertification. International Journal of Sustainable Development \& World Ecology, 17(2), 109-119.

Yang, X., Zhang, K., Jia, B., \& Ci, L. (2005). Desertification assessment in China: An overview. Journal of Arid Environments, 63(2), 517-531.

Yassoglou, N., Montanarella, L., Govers, G., Van Lynden, G., Jones, R. J. A., Zdruli, P., .... King, D. (1998). Soil Erosion in Euope. European soil Bureau.

Yuan, D., Worthy, D., \& Nassersharif, B. (1994). Progress towards an intelligent image classification system using both remotely sensed and digital elevation model data. Paper presented at the International Conference on Computing in Environmental Managment, Raleigh, NC. 
